\title{
Cognitive and electrophysiological responses to dopaminergic manipulations
}

Citation for published version (APA):

Linssen, A. M. W. (2013). Cognitive and electrophysiological responses to dopaminergic manipulations.

[Doctoral Thesis, Maastricht University]. Maastricht University. https://doi.org/10.26481/dis.20130118al

Document status and date:

Published: 01/01/2013

DOI:

10.26481/dis.20130118al

Document Version:

Publisher's PDF, also known as Version of record

\section{Please check the document version of this publication:}

- A submitted manuscript is the version of the article upon submission and before peer-review. There can be important differences between the submitted version and the official published version of record.

People interested in the research are advised to contact the author for the final version of the publication, or visit the DOI to the publisher's website.

- The final author version and the galley proof are versions of the publication after peer review.

- The final published version features the final layout of the paper including the volume, issue and page numbers.

Link to publication

\footnotetext{
General rights rights.

- You may freely distribute the URL identifying the publication in the public portal. please follow below link for the End User Agreement:

www.umlib.nl/taverne-license

Take down policy

If you believe that this document breaches copyright please contact us at:

repository@maastrichtuniversity.nl

providing details and we will investigate your claim.
}

Copyright and moral rights for the publications made accessible in the public portal are retained by the authors and/or other copyright owners and it is a condition of accessing publications that users recognise and abide by the legal requirements associated with these

- Users may download and print one copy of any publication from the public portal for the purpose of private study or research.

- You may not further distribute the material or use it for any profit-making activity or commercial gain

If the publication is distributed under the terms of Article $25 \mathrm{fa}$ of the Dutch Copyright Act, indicated by the "Taverne" license above, 
Cognitive and Electrophysiological Responses

tO DOPAMINERGIC MANIPULATIONS 
Colophon

(c) A.M.W. Linssen, Maastricht 2012 ISBN 978-94-6203-257-6

Photography: Pierre Linssen

Printing: Wöhrmann Print Service, Zutphen, The Netherlands 


\section{Cognitive ANd Electrophysiological ReSPONSES tO DOPAMINERGIC MANIPULATIONS}

\section{PROEFSCHRIFT}

Ter verkrijging van de graad van doctor aan de Universiteit Maastricht, op gezag van de Rector Magnificus, Prof. Dr. L.L.G. Soete volgens het besluit van het College van Decanen, in het openbaar te verdedigen op vrijdag 18 januari 2013 om 10.00 uur

$$
\text { door }
$$

Anna Maria Wilhelmina (Anke) Linssen 


\section{Promotor:}

Prof. Dr. W.J. Riedel

\section{Co-promotores:}

Dr. E.F.P.M. Vuurman

Dr. A. Sambeth

\section{Beoordelingscommissie:}

Prof. Dr. J.G. Ramaekers (voorzitter)

Prof. Dr. J.M.A. van Gerven (Leiden University Medical Center)

Prof. Dr. J. van Os

Dr. F.T. Smulders 


\section{TABLE OF CONTENTS}

$\begin{array}{ll}\text { General Introduction } & 7\end{array}$

Part 1: The role of dopamine in cognition 13

Part 1a: The effect of dopamine enhancement on cognition 15

Chapter 1. Cognitive effects of methylphenidate in healthy volunteers: A review

Chapter 2. Methylphenidate produces selective enhancement of declarative memory consolidation in healthy volunteers

Chapter 3. Effects of methylphenidate and levodopa on (working) memory performance and Event Related Potentials in healthy volunteers

Part 1b: The effect of reduced dopamine levels on cognition

Chapter 4. Effects of tyrosine/phenylalanine depletion on electrophysiological correlates of memory in healthy volunteers

Part 2: The role of dopamine in Psychomotor function

Chapter 5. Higher, faster stronger: The effect of dynamic stimuli on response preparation and CNV amplitude

Chapter 6. Contingent Negative Variation as a dopaminergic biomarker: Evidence from dose-related effects of methylphenidate

Chapter 7. Effects of methylphenidate and levodopa on the

Contingent Negative Variation

General discussion

References

Summary

Samenvatting

Dankwoord

Curriculum Vitae 

General Introduction 
Dopamine was discovered as a brain neurotransmitter by Nobel prize winner Arvid Carlsson almost 60 years ago (Iversen \& Iversen, 2007). Since the discovery of the role of dopamine in Parkinson's Disease, several other neuropsychiatric diseases have been associated with dopamine dysfunction, including schizophrenia, Attention Deficit Hyperactivity Disorder (ADHD), Alzheimer's Disease and Huntington's Disease (Nieoullon, 2002). Levodopa was discovered as a treatment for Parkinson's Disease more than 40 years ago and is still considered the most efficacious one. Early developments in pharmacotherapy of schizophrenia, demonstrating effectiveness of dopamine antagonists against psychosis meant a major improvement in treatment of this disease (Marsden \& Parkes, 1977). However, later advances have been disappointing. In spite of progress in knowledge and techniques in basic neuroscience, relatively few novel medications have been introduced on the market (Markou, Chiamulera, Geyer, Tricklebank, \& Steckler, 2009; SamsDodd, 2005). Hence, psychopharmacological research aiming at improvement of treatment of diseases that are associated with dysfunction of the dopamine neurotransmitter system continues to be highly relevant. A first step in the development of new dopaminergic agents is to increase our knowledge about the dopamine system.

\section{Dopamine in the brain}

Dopamine is a so-called catecholamine, meaning it has a catechol nucleus to which an amine group is attached (Julien, 2011). Noradrenaline is also classified as a catecholamine. The first step in dopamine synthesis encompasses the derivation of tyrosine from the amino acid phenylalanine. Tyrosine is transformed into L-Dopa, which is converted to dopamine (Mehta \& Riedel, 2006). Dopamine can in turn be converted into noradrenaline and finally adrenaline. Dopamine synthesis and release are controlled by enzymes, genetic influences and autoreceptors (Julien, 2011).

Released dopamine binds to postsynaptic receptors which are categorized based on their mechanism of action. The two main categories are D1 and D2. The D1 receptors are further subdivided in D1 and D5 subtypes, and the D2 family is subdivided in D2S, D2L, D3 and D4 receptor subtypes (Missale, Nash, Robinson, Jaber, \& Caron, 1998). Removal of dopamine from the synaptic cleft occurs through enzymatic breakdown by monoamine oxidase (MAO) and catechol-o-methyltransferase (COMT) and binding to the dopamine transporter (Julien, 2011; Mehta \& Riedel, 2006). Virtually each of these steps in neurotransmission, from synthesis, via release to enzymatic breakdown and reuptake can be targeted by pharmacological manipulations that regulate dopamine activity, constituting possible treatment options and/or models of neurotransmitter (dys)function. 
Dopamine pathways in the brain originate in the brainstem. These pathways are each associated with different processes. The tuberoinfundibular pathway from the hypothalamus to the pituitary gland is involved in hormonal regulation. In Parkinson's Disease, neuronal loss is observed in the nigrostriatal pathway, which plays a major role in motor control. The meso-cortico-limbic pathways are implicated in cognitive control, including motivation, reward, punishment and (working) memory (Cools, 2008). Hence, the widespread innervation of the brain by the various dopamine pathways is reflected by the diversity of functions in which dopamine is thought to play a role.

In Parkinson's disease, progressive loss of dopamine neurons eventually leads to severe motor symptoms including bradykinesia, muscle rigidity, resting tremor, and impairment of postural balance. However, more subtle effects of dopamine on psychomotor control can also be observed both in early stage Parkinson's Disease patients as well as healthy volunteers and be detected by with a response preparation paradigm. Preparation of a motor response has been associated with dopamine release in the basal ganglia. Its electrophysiological correlate, the Contingent Negative Variation (CNV) amplitude is reduced in Parkinson's Disease and varies with dopamine activity in healthy individuals (Amabile, et al., 1986; Oishi, Mochizuki, Du, \& Takasu, 1995; Tecce, 1991).

A first indication of dopamine involvement in cognitive function was also provided by the deficits observed in Parkinson's Disease patients. These patients display deficits in visuospatial abilities and executive function, including working memory and planning (Azuma, Cruz, Bayles, Tomoeda, \& Montgomery, 2003). Dopamine level enhancement by levodopa treatment improves cognitive functions that depend on brain areas that are dopamine depleted in Parkinson's Disease while functions depending on non-depleted areas deteriorate (Cools, 2006). These results can be explained in terms of the inverted-U relationship between dopamine and performance described in the literature, in which performance is mapped as a function of degree of neurotransmitter activity (Cools, 2006; Cools, Barker, Sahakian, \& Robbins, 2001). In an inverted-U relation, intermediate levels of neurotransmitter activity lead to optimal cognitive performance, but lower and higher levels may lead to suboptimal performance (Husain \& Mehta, 2011).

It is unclear if normal dopamine levels in the healthy brain are optimal for cognitive performance and the question arises to what extent cognitive performance may be improved by dopaminergic manipulations. This issue is currently relevant in the context of the vast increase in the illicit use of prescription drugs in attempts to enhance normal cognitive performance. Methylphenidate, a catecholamine reuptake inhibitor prescribed for ADHD and 
Narcolepsy is more and more used by for example students and academic staff with the intention to enhance their cognitive performance (Maher, 2008). If this is acceptable and if so, in what context, is an interesting discussion in itself. However first of all, it is important to find out if cognitive performance can indeed be enhanced by dopaminergic drugs.

\section{Aims}

1. After more than 50 years of dopamine research, there is still a lot to be learned about the dopamine system. Studies in clinical conditions, including Parkinson's Disease, have suggested a role for dopamine in cognition. The role of dopamine in cognitive processes in healthy volunteers is less clear. Moreover, recent increases in illicit use of prescription drugs such as methylphenidate by healthy individuals have raised interest in the question whether dopaminergic substances can enhance cognitive function. The first aim of this thesis is to determine the role of dopamine in cognitive function in the healthy population, primarily focusing on (working) memory.

2. Dopamine is involved in several debilitating neuropsychiatric conditions including Parkinson's Disease, schizophrenia and ADHD. To advance pharmacological treatment of these diseases, further research is needed. This research would be highly aided by a biomarker for dopamine activity. This thesis proposes the use of CNV amplitude, which is under the influence of changes in dopamine availability, as a marker of dopamine activity in the brain. The second aim of this thesis is, therefore, to validate the CNV paradigm and fine-tune its' application as a dopaminergic biomarker.

\section{Methods}

In this thesis, the relationship between dopamine and cognitive function in healthy individuals is investigated by measuring performance on cognitive tests while dopamine activity is modulated, using a placebo controlled crossover design. Effects of both increased dopamine turnover as well as reduced dopamine activity on performance are studied.

In the studies described in this thesis, dopamine availability is enhanced by administration of methylphenidate and levodopa. Methylphenidate binds to the dopamine transporter and thereby inhibits reuptake of the neurotransmitter from the synaptic cleft. Levodopa is the precursor of dopamine. It can cross the blood-brain barrier, and is transformed into dopamine.

A reduction of dopamine activity is achieved by depleting the brain of tyrosine and phenylalanine, the amino acids that form the building blocks for 
dopamine synthesis (Barrett \& Leyton, 2004). Because these amino acids are transported into the brain by neutral amino acid transporters, amino acids have to compete with each other for binding to transporters. Lowering the relative amount of one or two specific amino acids worsens their position in this competition, which leads to decreased availability of these precursors in the brain (McTavish, et al., 2001).

To validate CNV as a dopaminergic biomarker, its dynamic range is studied under normal conditions compared with pharmacologically increased dopamine availability. The relationship between dopamine and psychomotor function is assessed with a response preparation paradigm, in which electrophysiological measurements are taken. From the electroencephalography (EEG), event-related potentials are extracted and the CNV amplitude can be determined. In addition, EEG measurements are taken during some of the cognitive tasks, to get a more comprehensive view of the brain functions (i.e. declarative and working memory) of interest.

\section{Outline of this thesis}

Part 1 discusses the role of dopamine in cognition. Both dopamine enhancement and reduction in dopamine activity are employed to study the relationship between dopamine and brain functions such as (working) memory, attention and executive function. Part 1a, consisting of Chapter 1, 2 and 3 , answers questions regarding the relationship between dopamine enhancement and cognitive function. Part $1 \mathrm{~b}$, Chapter 4 , examines the effect a reduction in dopamine activity has on cognitive processes.

\section{Chapter 1}

The first chapter gives an overview of the literature on the cognitive effects of methylphenidate in healthy volunteers. Methylphenidate's effects on six cognitive subdomains are quantified in relation to the quality of the studies reviewed. In using this approach we aimed to find out if methylphenidate selectively affects specific subdomains of cognitive function.

\section{Chapter 2}

This chapter describes an experimental study in which dose related effects of methylphenidate on declarative memory, working memory, attention and executive functions are examined.

\section{Chapter 3}

To elucidate whether the effects of methylphenidate on cognitive function reported in chapter 2 are mediated by the dopaminergic or noradrenergic neurotransmitter system, the experimental study described in chapter 3 compared the effects of methylphenidate to the purely dopaminergic effects of levodopa on cognitive function in healthy volunteers. 


\section{Chapter 4}

In contrast with the preceding three chapters, chapter 4 describes an experiment with a manipulation leading to a decrease in the level of dopamine. This is achieved by tyrosine/phenylalanine depletion; the administration of an amino acid mixture excluding the building blocks for dopamine synthesis. Effects of reduced dopamine activity on declarative memory and working memory and their electrophysiological correlates are studied.

Part 2 focuses on psychomotor control and how it is mediated by dopamine. Psychomotor function is assessed by response preparation tasks which induce the Contingent Negative Variation. The studies in this part aim to validate the use of the CNV as a dopaminergic biomarker.

\section{Chapter 5}

The first chapter of Part 2 studies differences between several paradigms that elicit a CNV. In an effort to optimize the CNV paradigm, a new task using dynamic stimuli was introduced. The aim of the first experiment was to see if dynamic stimuli would lead to increased attention and motivation and induce a larger amplitude of the CNV wave, compared to a task with static stimuli. The second experiment investigates how variation in response requirement affects the CNV wave and task performance.

\section{Chapter 6}

Chapter 6 aimed to determine the sensitivity of CNV amplitude to dopamine activity and hence, if it could serve as a pharmacodynamic marker of dopamine activity. To this end the dose related effects of methylphenidate on the static and dynamic response preparation paradigms described in chapter 5 are investigated in chapter 6.

Chapter 7 As methylphenidate does not only affect dopamine but also noradrenaline activity, the final chapter investigates the specific dopaminergic influence on the CNV amplitude by comparing the effects of methylphenidate on CNV to those of levodopa.

\section{General discussion}

In the general discussion, the findings from the individual chapters are reviewed to form a comprehensive view with respect to the two central aims of this thesis. The role of dopamine in cognitive function in the healthy population is discussed. Furthermore, the relationship between dopamine and the CNV is described. It is evaluated whether CNV amplitude could serve as a dopaminergic biomarker. 
PART 1: The ROLE OF DOPAMINE IN COGNITION 

Part 1a: The effect of dopamine enhancement on Cognit ion 

Chapter 1. Cognitive effects of methylphenidate in healthy volunteers: $A$ review

Submitted for publication

\begin{abstract}
Methylphenidate (MPH), a stimulant drug with dopamine and noradrenaline reuptake inhibition properties is mainly prescribed in attention deficit hyperactivity disorder, is increasingly used by the general population, intending to enhance their cognitive function. We aim to answer whether this is effective and present a novel way to determine the extent to which MPH enhances cognitive performance in a certain domain. Namely, we quantify this by a percentage that reflects the number of studies showing positive effects of MPH. To evaluate whether the dose-response relationship follows an inverted-U-shaped curve, MPH effects on cognition are also quantified for low, medium and high doses respectively. The studies reviewed here show that MPH improves cognitive performance in the healthy population in the domains of working memory (68\% of included studies) and speed of processing $(61 \%)$, and to a lesser extent may also improve verbal learning and memory $(34 \%)$, reasoning and problem solving $(18 \%)$ and attention and vigilance $(16 \%)$, but does not have an effect on visual learning and memory. MPH effects are dose dependent and the dose response relationship differs between cognitive domains. In conclusion, healthy individuals should be very careful when considering using MPH for cognition enhancing purposes; effects may be disappointing to abusers.
\end{abstract}




\section{Introduction}

Methylphenidate (MPH, also see Table 1 for an overview of abbreviations used in this article) is a stimulant drug that is mainly prescribed in attention deficit hyperactivity disorder (ADHD, (Leonard, McCartan, White, \& King, 2004)). In the past decades there has been a vast increase in the use of MPH (Diller, 1996). Not only by patients to whom MPH is prescribed, but also by the general population, who believe MPH enhances their cognitive functions (Maher, 2008). Especially the latter has raised ethical concern (Larriviere, Williams, Rizzo, \& Bonnie, 2009; Sahakian \& Morein-Zamir, 2007; Stix, 2009). Discussions in popular scientific literature consider safety, potential abuse and side effects (Stix, 2009; Swanson \& Volkow, 2008) of cognition enhancing drugs. However, even if these issues were no reason for concern, a critical question would still be: 'How effective are supposed cognition enhancing drugs actually?'. Although there are some indications that MPH may improve certain aspects of cognitive function (e.g. working memory (Mehta, et al., 2000)), there are also reports of impaired performance on cognitive tasks after MPH (e.g. planning (Elliott, et al., 1997)). While a reasonable amount of studies have examined cognitive effects of MPH in healthy volunteers, the evidence is undeniably mixed.

Cognitive effects of MPH in children with ADHD have been reviewed by Pietrzak et al. (2006). Their extensive review includes evidence on a broad range of cognitive functions and presents it classified into several cognitive domains. An equivalent review on cognitive effects of $\mathrm{MPH}$ in healthy volunteers is not available yet. Previous reviews on the cognition enhancing effects of drugs have focused on attention, learning, memory and executive function (Repantis, Schlattmann, Laisney, \& Heuser, 2010; M. E. Smith \& Farah, 2011). In the current review the full spectrum of cognitive function is considered. As Pietrzak et al. (2006) also argue, the differences between studies on cognitive effects of MPH, such as differences in methodology, MPH dose, neuropsychological instruments employed do not encourage conducting a meta-analysis. As we did not want to limit our review to one particular measure or cognitive domain, we used a similar approach as Pietrzak et al. (2006). However,because the review by Pietrzak et al. (2006) focused on studies involving children with ADHD, the reported tasks differ slightly from those in studies with healthy volunteers, since both clinical status (i.e. ADHD diagnosis) and age (children vs. adults) may call for a different selection of tasks. Inspection of the range of tasks used in studies with MPH in healthy volunteers, and the fact that the classification applied by Pietrzak et al. (2006) does not seem to be based on factor analysis or any other established way of classifying cognitive tasks (or is at least not reported to be) led us to 
look for a classification that was more appropriate for the available data (Pietrzak, Mollica, Maruff, \& Snyder, 2006).

Table 1

\begin{tabular}{ll} 
Abbreviations & \\
\hline ADHD & attention deficit hyperactivity disorder \\
A/V & attention/vigilance \\
CNV & contingent negative variation \\
CPT & continuous performance test \\
DSST & digit symbol substitution \\
ID/ED & intra-/extradimensional shift task \\
MPH & Methylphenidate \\
PAL & paired associates learning \\
PASAT & paced auditory serial addition test \\
RPS & reasoning and problem solving \\
RT & response time \\
RVIP & rapid visual information processing \\
SERS & stimulus evaluation/response selection task \\
SMS & Sternberg memory scanning task \\
SoP & speed of processing \\
TMT & trail making test \\
TOVA & test of variables of attention \\
VEM & Verbal learning and memory \\
VSM & Visual learning and memory \\
WLT & Word learning test \\
WM & Working memory \\
\hline
\end{tabular}

The categorization based on the Identification of separable cognitive factors in schizophrenia by a committee of the National Institute of Mental Health (NIMH) (Nuechterlein, et al., 2004) is based on a number of factor analytic studies and fine-tuned by a committee of field experts. Although the identification considered cognitive function in schizophrenia patients, it is generally accepted that cognitive domains are broadly the same in healthy controls (Dickinson, Ragland, Calkins, Gold, \& Gur, 2006; Genderson, et al., 2007). Since the cognitive domains (i.e. speed of processing, attention/vigilance, working memory, verbal learning and memory, visual learning and memory and reasoning and problem solving) fit well with the literature on healthy volunteers, we decided to employ this in the current review (Riedel, Mehta, \& Unema, 2006).

Considering the mixed nature of the literature, a descriptive statistic quantifying the evidence would facilitate its interpretation. Pietrzak et al. 
(2006) summarize the results within each domain as a percentage of studies reporting cognition enhancing effects of $\mathrm{MPH}$. This does, however, not take into account study characteristics such as the number of subjects tested or correction for multiple comparisons. The result of a study testing a high number of subjects has more statistical power than a study with a low number of subjects. Therefore, it is important to weigh studies according to the number of subjects (Kleijnen, Knipschild, \& Riet ter, 1991). Furthermore, a study including many tests is more likely to find a result than a study assessing performance on just one test. In order to take these factors into account, we established a weighed percentage reflecting the contribution of each task/measure in each study to the effect of MPH on cognitive function. Details on the calculation of this percentage can be found in the method section.

Effects of dopamine on cognition are often described to follow an inverted-U-shaped curve in which intermediate levels of neurotransmitter activity lead to optimal cognitive performance, but lower and higher levels may lead to suboptimal performance (Husain \& Mehta, 2011). Since MPH blocks the dopamine and noradrenaline transporters, thereby blocking reuptake of these neurotransmitters, MPH leads to increased levels of dopamine and noradrenaline availability (Hannestad, et al., 2010; Volkow, Wang, Fowler, Gatley, et al., 1998) and may demonstrate inverted-U properties. Moreover, dose-response relationships may vary between cognitive domains. To evaluate the dose-response relationship between MPH and its effects on the different cognitive domains we report a weighed percentage reflecting $\mathrm{MPH}$ effects, equivalent to the one described above for low, medium and high doses respectively (see method section for more details).

The aim of this review is to answer the question whether MPH can enhance cognitive function in healthy individuals. Unlike previous reviews on MPH effects of cognitive function in healthy individuals, this review specifically looks at the acute effects of MPH on healthy adults (i.e. excluding effects of other medications, effects on children or the elderly, effects after sleep deprivation and long term effects). Further improvements compared to earlier reviews include: (1) the results from the studies included in this review are carefully categorized in cognitive domains; and: (2) the extent to which MPH affects each domain is quantified in relation to the studies' quality. Although we are aware of the ethical issues involved, these are not further considered here. In this review we give an overview of the relevant literature and report the extent to which MPH enhances cognitive performance within each cognitive domain. 


\section{Method}

\section{Literature search}

A literature search was performed in PubMed and Psychinfo using the search term Methylphenidate combined with the terms cognition, neuropsychology, executive, working memory, vigilance and inhibition in separate searches. Reference lists of extracted articles were screened for omissions in our search. Studies that were included fulfilled the following criteria: used immediate release formulation of $\mathrm{MPH}$; assessed healthy human adults aged between 18 and 60 years of age (of studies including older participants, only data of participants aged between 18-60 were included in this review); assessed the effects using cognitive tasks; were written in English; and were published in a peer-reviewed journal. The earliest study included was published March 1978 and the last included study was published June 2012.

\section{Categorization}

The neuropsychological tests were categorized into 6 different cognitive domains: speed of processing, attention/vigilance, working memory, verbal learning and memory, visual learning and memory and reasoning and problem solving (Nuechterlein, et al., 2004). Tasks employed in the included articles were categorized following the classification by Nuechterlein et al. (2004). If more than one cognitive domain was applicable, tasks were still classified as measuring one domain only (i.e. the most salient domain). Tasks that could not be categorized in any of these 6 cognitive domains were not included in the analysis (for instance 'spatial bias' in (Dodds, Muller, \& Manly, 2008).

\section{Outcome measure}

For each domain a weighed percentage was calculated reflecting to what extent MPH affects task performance in that specific domain. It was calculated as follows:

Outcome measure $=(\Sigma$ (number of participants * outcome significance testing ${ }^{*}$ relative contribution $\left.)\right) /(\Sigma$ (number of participants * relative contribution)) ${ }^{*} 100 \%$

in which outcome significance testing was defined as: 1 = significantly improved task performance; -1 = significantly impaired task performance; $0=$ no significant effect; $0.5=$ trend towards improved task performance; $-0.5=$ trend towards impaired task performance and the factor to correct for multiple comparisons (relative contribution) was the defined as: $1 /$ number of tasks or measures within the study that are reported in Table 2 (i.e. if 3 measures were reported in the article, but only 2 were listed in Table 2, the factor was $1 / 2=0.5$ ). 


\section{Dose effects}

The outcome measure as detailed above was also calculated for low, medium and high doses separately. A low, medium or high dose was defined as follows: low: $<=10 \mathrm{mg}$ or $<=0.15 \mathrm{mg} / \mathrm{kg}$; medium: $>10 \mathrm{mg},<=20 \mathrm{mg}$ or $>0.15$ $\mathrm{mg} / \mathrm{kg},<=0.3 \mathrm{mg} / \mathrm{kg}$; high: $>20 \mathrm{mg}$ or $>0.3 \mathrm{mg} / \mathrm{kg}$. If it was not clearly stated which dose(s) led to significant effects, it was assumed that the reported effect was applicable to every administered dose.

\section{Results and discussion}

In total 45 studies met the inclusion criteria. These studies are listed in Table 2. The extent to which MPH enhances cognitive performance (quantified by a weighed percentage) is reported in Table 3. The results show that MPH most effectively enhances performance in the domain of working memory. Second most affected were tasks measuring speed of processing, followed by verbal learning and memory, reasoning and problem solving, attention/vigilance and visual learning and memory. In the next few paragraphs, the results per domain are discussed in more detail.

\section{Working memory}

Working memory involves the temporary storage and manipulation of information (Baddeley, 1992). For the purpose of this review, no distinction will be made between short term memory and working memory tasks. A common working memory task is the digit span, requiring subjects to repeat a sequence of digits either in the exact same or reversed order. More complex tasks include memory scanning tasks and N-back tasks. Memory scanning tasks present participants with probes that they have to compare to a memory set that may vary in size, and hence, memory load (Sternberg, 1966). N-back tasks require a response to stimuli that are the same as the stimulus that was presented $\mathrm{N}$-trials before. Other tasks specifically assess spatial working memory, which is often associated with dopaminergic activity (Mehta \& Riedel, 2006).

Although the number of MPH studies including a test of working memory is not very high, the proportion that shows enhancing effects in this cognitive domain is the highest of all reported domains, namely $68 \%$. This can not be attributed to MPH effects on a specific type of test, but reflects the general pattern across a variety of tests. For example, there are four reports of scanning tests, three of which observe improved performance after MPH (Brumaghim, Klorman, Strauss, Lewine, \& Goldstein, 1987; Fitzpatrick, Klorman, Brumaghim, \& Keefover, 1988; Halliday, Callaway, Naylor, Gratzinger, \& Prael, 1986). Similarly, three of the five spatial working 
memory assessments showed enhancing effects. Better performance was observed after 10, 20 and $40 \mathrm{mg}$ of $\mathrm{MPH}$ on the spatial span test (requiring subjects to reproduce a spatial sequence that is presented) and on a selfordered search task in which participants search for tokens in an array of boxes displayed on a computer screen (Elliott, et al., 1997; Mehta, et al., 2000). The latter task was also employed by Clathworthy et al. (2009) but performance was not improved in this study, in which $60 \mathrm{mg}$ of MPH was administered. An object relocation task, which assesses spatial working memory, was also not affected by 10,20 and $40 \mathrm{mg}$ of MPH (Linssen, Vuurman, Sambeth, \& Riedel, 2012).

Two studies, both using medium doses of $\mathrm{MPH}$, assessed digit span performance, one reporting improved performance and one reporting no drug effects (Agay, Yechiam, Carmel, \& Levkovitz, 2010; Oken, Kishiyama, \& Salinsky, 1995). A medium dose also lead to faster response times on an $\mathrm{N}$ back task, but did not improve accuracy on this task (Tomasi, et al., 2011).

Two other studies did not observe any effect of MPH on working memory task performance, using a medium dose (Kuypers \& Ramaekers, 2005; Studer, et al., 2010). The task used by Kuypers and Ramaekers (2005) involved deciding whether the presented sentences describing the order of two letters, A and B, corresponded to the presented letterpairs. Studer et al. (2010) used a task in which pictures were presented and participants were probed with the same pictures in the retention phase and had to indicate what position in the sequence the probe was in.

\section{Speed of Processing}

Tasks that are classified as a measure of speed of processing are relatively simple, involving fundamental processes such as perception and motor action. This category includes for example Digit symbol substitution (DSST) and Trail making tests (TMT; version A and B). In the former, participants are asked to complete as many digit/symbol pairings as they can within 90 seconds. The TMT test involves connecting letters and/or numbers in ascending order. A task employed by some older studies, the stimulus evaluation/response selection (SERS) task, requires fast responses to a target stimulus that is either presented alone or surrounded by distracter stimuli. Different trials may either require a simple key press or the selection of the correct key, depending on the location of the target stimulus.

The speed of processing domain was with $61 \%$ the second most affected domain in terms of performance enhancing effects of $\mathrm{MPH}$. This is consistent with the notion that $\mathrm{MPH}$ speeds response time in healthy volunteers (Elliott, et al., 1997), reflecting response readiness enhancing effects (Linssen, et al., 2011). Tasks requiring participants to press (a series 
Summary of studies on cognitive effects of MPH in healthy volunteers

\begin{tabular}{|c|c|c|c|c|c|}
\hline Study & $\begin{array}{l}\text { Nr. of pp M, F (age) } \\
\text { design }\end{array}$ & Dose & Task/measure & Domain & Effect \\
\hline Agay et al. (2010) & $18 \mathrm{M}, 14 \mathrm{~F}(32.7)$ & $15 \mathrm{mg}(1)$ & CPT (omission errors) & $\mathrm{A} / \mathrm{V}$ & ns \\
\hline \multirow[t]{2}{*}[1]{} & placebo controlled & & CPT (commission errors) & $A / V$ & ns \\
\hline & between groups & & Digit span & WM & sig \\
\hline Aman et al. (1984) & $5 M, 7 F(28.3)$ & $0.3 \mathrm{mg} / \mathrm{kg}$ & CPT (omission errors) & $\mathrm{A} / \mathrm{V}$ & ns \\
\hline \multirow[t]{2}{*}[2]{} & placebo controlled & & CPT (commission errors) & $\mathrm{A} / \mathrm{V}$ & sig \\
\hline & crossover & & CPT (RT) & $\mathrm{A} / \mathrm{V}$ & ns \\
\hline Anderer et al. (2002) & $10 \mathrm{M}, 10 \mathrm{~F}(23-34)$ & $20 \mathrm{mg}$ & Oddball (error rate) & $\mathrm{A} / \mathrm{V}$ & ns \\
\hline$[3]$ & $\begin{array}{l}\text { placebo controlled } \\
\text { crossover }\end{array}$ & & & & \\
\hline Bishop et al. (1997) & $6 M, 3 F(21-35)$ & $10 \mathrm{mg}(2 \mathrm{x} /$ day $)$ & divided attention & $\mathrm{A} / \mathrm{V}$ & ns \\
\hline$[4]$ & $\begin{array}{l}\text { placebo controlled } \\
\text { crossover }\end{array}$ & & auditory vigilance & $\mathrm{A} / \mathrm{V}$ & ns \\
\hline Brignell et al. (2006) & $30 \mathrm{HV}(23.5)$ & $40 \mathrm{mg}$ & fear conditioning & VSM & ns \\
\hline$[5]$ & $\begin{array}{l}\text { placebo controlled } \\
\text { between groups }\end{array}$ & & & & \\
\hline $\begin{array}{l}\text { Brignell et al. (2007) } \\
\text { [6] }\end{array}$ & $\begin{array}{l}32 \mathrm{HV}(22.8) \\
\text { placebo controlled } \\
\text { between groups }\end{array}$ & $40 \mathrm{mg}$ & story task & VEM & trend $(2)$ \\
\hline $\begin{array}{l}\text { Brumaghim et al. (1998) } \\
\text { [7] }\end{array}$ & $\begin{array}{l}12 \mathrm{M}, 20 \mathrm{~F}(20.9) \\
\text { placebo controlled } \\
\text { crossover }\end{array}$ & $0.3 \mathrm{mg} / \mathrm{kg}$ & PAL (CVC pairs) & VEM & ns \\
\hline Brumaghim et al. (1987) & $19 M(19.4)$ & $0.3 \mathrm{mg} / \mathrm{kg}$ & SMS & WM & sig \\
\hline
\end{tabular}


study 1

[8]

Brumaghim et al. (1987) study 2

[8]

Callaway (1984)

[9]

Camp-Bruno et al(1994)

[10]

Clark et al. (1986a)

[11]

Clark et al. (1986b)

[12] placebo controlled

crossover

$6 \mathrm{M}, 8 \mathrm{~F}(20.0)$

placebo controlled

crossover

8F (30-40)

placebo controlled

crossover

22M, 26F(23.0)

placebo controlled

between groups

$12 \mathrm{M}(18-30)$

placebo controlled

crossover

$0.65 \mathrm{mg} / \mathrm{kg}$

$20 \mathrm{mg}$

CPT (RT)

CPT (sensitivity)

CPT (In beta)

Word learning

Buschke selective reminding

Dichotic monitoring task

(target detection)

Dichotic monitoring task (error rate)

Dichotic monitoring task (RT)

Dichotic monitoring task

(signal detection)

$0.65 \mathrm{mg} / \mathrm{kg}$

$10 \mathrm{M}(18-30)$

placebo controlled

Dichotic monitoring task

(target detection)

Dichotic monitoring task (error rate)

Dichotic monitoring task (RT)

Dichotic monitoring task

(signal detection)
WM sig

SoP sig

A/V sig

A/V ns

A/V ns

VEM ns

VEM sig

A/V ns

$\mathrm{A} / \mathrm{V}$ ns

$\mathrm{A} / \mathrm{V}$ ns

A/V ns

A/V ns

A/V sig

A/V ns

A/V ns 


\begin{tabular}{|c|c|c|c|c|c|}
\hline Study & $\begin{array}{l}\text { Nr. of pp M, F (age) } \\
\text { design }\end{array}$ & Dose & Task/measure & Domain & Effect \\
\hline Clatworthy (2009) & $10 M(22-32)$ & $60 \mathrm{mg}$ & Reversal learning & VSM & ns \\
\hline$[13]$ & $\begin{array}{l}\text { placebo controlled } \\
\text { crossover }\end{array}$ & & Spatial WM & WM & ns \\
\hline Coons et al. (1981) & $13 \mathrm{M}(23.84)$ & $20 \mathrm{mg}$ & CPT X (omission errors) & $\mathrm{A} / \mathrm{V}$ & ns \\
\hline study 1 & placebo controlled & & CPT X (commission errors) & $\mathrm{A} / \mathrm{V}$ & ns \\
\hline \multirow[t]{2}{*}[14]{} & crossover & & CPT BX (omission errors) & $\mathrm{A} / \mathrm{V}$ & ns \\
\hline & & & CPT BX (commission errors) & $\mathrm{A} / \mathrm{V}$ & ns \\
\hline Coons et al. (1981) & $23 M(19.7)$ & $20 \mathrm{mg}$ & CPT X (omission errors) & $\mathrm{A} / \mathrm{V}$ & ns \\
\hline study 2 & placebo controlled & & CPT X (commission errors) & $\mathrm{A} / \mathrm{V}$ & ns \\
\hline \multirow[t]{4}{*}[14]{} & crossover & & CPT BX (omission errors) & $\mathrm{A} / \mathrm{V}$ & sig \\
\hline & & & CPT BX (commission errors) & $\mathrm{A} / \mathrm{V}$ & ns \\
\hline & & & CPT Double (omission errors) & $\mathrm{A} / \mathrm{V}$ & sig \\
\hline & & & $\begin{array}{l}\text { CPT Double (commission errors) } \\
\text { Oddball (omission errors) } \\
\text { Oddball (commission errors) } \\
\text { Choice RT (omission errors) } \\
\text { Choice RT (commission errors) } \\
\text { Choice RT (response time) }\end{array}$ & $\begin{array}{l}\text { A/V } \\
\text { A/V } \\
\text { A/V } \\
\text { SoP } \\
\text { SoP } \\
\text { SoP }\end{array}$ & $\begin{array}{l}\text { ns } \\
\text { ns } \\
\text { ns } \\
\text { sig } \\
\text { ns } \\
\text { ns }\end{array}$ \\
\hline Cooper et al. (2005) & $32 \mathrm{M}(22.3)$ & $5 / 15 / 45 \mathrm{mg}$ & CPT (omission errors) & $\mathrm{A} / \mathrm{V}$ & sig \\
\hline \multirow[t]{2}{*}{ [15] } & placebo controlled & & CPT (commission errors) & $\mathrm{A} / \mathrm{V}$ & ns \\
\hline & crossover & & CPT (RT)/N back & $\mathrm{A} / \mathrm{V}$ & sig \\
\hline $\begin{array}{l}\text { Dodds et al. (2008) } \\
{[16]}\end{array}$ & $\begin{array}{l}14 \mathrm{M}, 6 \mathrm{~F}(22.2) \\
\text { placebo controlled } \\
\text { crossover }\end{array}$ & $60 \mathrm{mg}$ & Probabilistic reversal learning & VSM & ns \\
\hline Elliott et al. (1997) & $28 M(21.3)$ & $20 / 40 \mathrm{mg}$ & Spatial WM & WM & $\operatorname{sig}(3)$ \\
\hline
\end{tabular}


Finke et al. (2010)

[18]

9M, 9F (20-35)

$40 \mathrm{mg}$

placebo controlled

crossover

Fitzpatrick et al. (1988)

[19]

20M (19.7)

placebo controlled

crossover

Halliday et al. (1986)

$\exp 1$

8F (30-40)

placebo controlled

crossover

[20]

Halliday et al. (1986)

$12 \mathrm{M} \mathrm{(26)}$

$\exp 2$

placebo controlled

[20]

crossover

Hermens et al. (2007)

[21]

Visual perceptual processing speed

A/V ns

Visual short-term memory storage

SoP sig

\section{capacity}

$0.3 \mathrm{mg} / \mathrm{kg}$

Memory scanning task

WM sig

\section{$5 / 10 / 20 \mathrm{mg}$}

SERS

SMS

SoP sig

WM ns

\section{SERS}

SoP sig

CPT (commission errors)

A/V sig

motor task

SoP sig

$5 / 15 / 45 \mathrm{mg}$

Oddball

CPT (RT)

CPT (omission errors)

A/V sig

A/V sig

A/V sig

CPT (commission errors)

A/V ns

CPT (total errors)

A/V sig

Maze

RPS ns 


\begin{tabular}{|c|c|c|c|c|c|}
\hline Study & $\begin{array}{l}\text { Nr. of pp M, F (age) } \\
\text { design }\end{array}$ & Dose & Task/measure & Domain & Effect \\
\hline & & & Mackworth clock (RT variability) & $\mathrm{A} / \mathrm{V}$ & sig \\
\hline & & & Mackworth clock (false negatives) & $\mathrm{A} / \mathrm{V}$ & sig \\
\hline & & & Mackworth clock (false positives) & $\mathrm{A} / \mathrm{V}$ & ns \\
\hline & & & Mackworth clock (total errors) & $\mathrm{A} / \mathrm{V}$ & sig \\
\hline & & & Verbal memory recall & VEM & ns \\
\hline & & & Choice reaction time & SoP & ns \\
\hline & & & Switching of attention (TMT A) & SoP & ns \\
\hline & & & Switching of attention (TMT B) & SoP & ns \\
\hline & & & PASAT (RT) & $\mathrm{A} / \mathrm{V}$ & sig \\
\hline & & & PAL (word pairs) & VEM & ns \\
\hline Hink et al. (1978) & $12 M(19-28)$ & $10 \mathrm{mg}$ & Target detection task & $A / V$ & ns \\
\hline$[22]$ & $\begin{array}{l}\text { placebo controlled } \\
\text { crossover }\end{array}$ & & & & \\
\hline Izquierdo et al. (2008) & $7 M, 5 F(40-74)$ & $10 \mathrm{mg}$ & Incidental memory task & VEM & sig \\
\hline$[23]$ & $\begin{array}{l}\text { placebo controlled } \\
\text { crossover }\end{array}$ & & & & \\
\hline Izquierdo et al. (2008) & $11 \mathrm{M}, 9 \mathrm{~F}(35-74)$ & $10 \mathrm{mg}$ & Formal memory task & VEM & sig \\
\hline$[23]$ & $\begin{array}{l}\text { placebo controlled } \\
\text { crossover }\end{array}$ & & & & \\
\hline Kollins et al. (1998) & $5 M, 5 F(30.7)$ & $20 / 40 \mathrm{mg}$ & DSST & SoP & ns \\
\hline$[24]$ & $\begin{array}{l}\text { placebo controlled } \\
\text { crossover }\end{array}$ & & circular lights task & SoP & sig \\
\hline Kratz et al. (2009) & $8 \mathrm{M}, 6 \mathrm{~F}(20-40)$ & $20 \mathrm{mg}$ & Go/No-go task (hit rate) & $\mathrm{A} / \mathrm{V}$ & ns \\
\hline$[25]$ & placebo controlled & & Go/No-go task (RT) & $\mathrm{A} / \mathrm{V}$ & sig \\
\hline
\end{tabular}


crossover

Kupietz et al. (1980)

[26]

Kuypers et al. (2005)

[27]

Kuypers et al.(2007)

[28]

Linssen et al. (2011)

[29]

Linssen et al. (2012)

[30]

Mehta et al. (2000)

[31]

Naylor et al.(1985)

[32]

Oken et al. (1995)

[33]

\section{M, 4F (28.7)}

placebo controlled crossover

9M, 9F (26.2)

placebo controlled crossover

9M, 9F (26.2)

placebo controlled crossover

19M (23.4)

placebo controlled crossover

19M (23.4)

placebo controlled crossover

$10 \mathrm{M}(34.8)$

$40 \mathrm{mg}$

placebo controlled

\section{crossover}

8F (30-39)

placebo controlled

crossover

$11 \mathrm{M}, 12 \mathrm{~F}$ (25)

placebo controlled
Impulsivity errors

A/V ns

\section{$5 / 10 \mathrm{mg}$}

Learning beginning reading

vocabulary (simultaneous method) VEM sig

(progressive method)

VEM ns

WLT

Syntactic resasoning task

VEM ns

DSST

WM ns

SoP ns

VSM ns

VSM ns

change blindness task

SoP sig

SoP sig

10/20/40 mg WLT

Spatial WM

Set shifting

Stop signal task

Tower of London

Spatial WM

VEM sig

WM ns

A/V sig

A/V sig

RPS ns

WM sig

$5 / 10 / 20 \mathrm{mg}$

SERS

SoP sig

$0.2 \mathrm{mg} / \mathrm{kg} \quad$ Covert orienting spatial attention

(RT) 


\begin{tabular}{|c|c|c|c|c|c|}
\hline Study & $\begin{array}{l}\text { Nr. of pp M, F (age) } \\
\text { design }\end{array}$ & Dose & Task/measure & Domain & Effect \\
\hline \multirow{13}{*}{$\begin{array}{l}\text { Roehrs et al. (1999) } \\
\text { [34] }\end{array}$} & \multirow[t]{6}{*}{ Crossover } & & (Errors) & $\mathrm{A} / \mathrm{V}$ & ns \\
\hline & & & Parallel visual search task (RT) & $\mathrm{A} / \mathrm{V}$ & ns \\
\hline & & & Parallel visual search task (Errors) & $\mathrm{A} / \mathrm{V}$ & ns \\
\hline & & & Serial visual search task (RT) & $\mathrm{A} / \mathrm{V}$ & ns \\
\hline & & & Serial visual search task (Errors) & $\mathrm{A} / \mathrm{V}$ & ns \\
\hline & & & Digit span & WM & ns \\
\hline & \multirow{7}{*}{$\begin{array}{l}2 \mathrm{M}, 4 \mathrm{~F}(21-30) \\
\text { placebo controlled } \\
\text { crossover }\end{array}$} & \multirow[t]{7}{*}{$10 \mathrm{mg}$} & \multicolumn{2}{|l|}{ Divided-attention task } & \\
\hline & & & (central RT) & $\mathrm{A} / \mathrm{V}$ & sig \\
\hline & & & (peripheral RT) & $\mathrm{A} / \mathrm{V}$ & ns \\
\hline & & & (tracking deviations) & $\mathrm{A} / \mathrm{V}$ & ns \\
\hline & & & \multicolumn{2}{|l|}{ auditory vigilance task } & \\
\hline & & & (mean RT) & $\mathrm{A} / \mathrm{V}$ & ns \\
\hline & & & (Errors) & $\mathrm{A} / \mathrm{V}$ & ns \\
\hline Rogers et al. (1999) & $32 M(20.5)$ & $40 \mathrm{mg}$ & ID/ED shift task & $\mathrm{A} / \mathrm{V}$ & sig (decreased \\
\hline$[35]$ & \multicolumn{2}{|l|}{$\begin{array}{l}\text { placebo controlled } \\
\text { between groups }\end{array}$} & & & performance) \\
\hline \multirow{3}{*}{$\begin{array}{l}\text { Rush et al. (2001) } \\
\text { [36] }\end{array}$} & $4 \mathrm{M}, 4 \mathrm{~F}(28)$ & \multirow[t]{3}{*}{$20 / 40 \mathrm{mg}$} & \multirow[t]{3}{*}{ DSST } & \multirow[t]{3}{*}{ SoP } & \multirow[t]{3}{*}{ ns } \\
\hline & placebo controlled & & & & \\
\hline & crossover & & & & \\
\hline Rush et al. (1998) & $2 M, 3 F(36)$ & \multirow[t]{3}{*}{$5 / 10 / 20 / 40 \mathrm{mg}$} & \multirow[t]{3}{*}{ DSST } & \multirow[t]{3}{*}{ SoP } & \multirow[t]{3}{*}{ ns } \\
\hline \multirow[t]{2}{*}[37]{} & placebo controlled & & & & \\
\hline & crossover & & & & \\
\hline Schroeder et al. (1987) & $10 M(18-40)$ & $0.15 / 0.30 \mathrm{mg} / \mathrm{kg}$ & Concurrent probability matching & & \\
\hline [38] & No placebo & & (hit rate) & RPS & ns \\
\hline
\end{tabular}


between groups

(changeover)

(strategy)

Stoops et al. (2005)

[39]

placebo controlled

crossover

Strauss et al. (1984)

[40]

22M (19.2)

placebo controlled

crossover

crossover

Studer et al. (2010)

[41]

Tomasi et al. (2011)

[42]

Unrug et al. (1997)

[43]

Volkow et al. (2008)

[44]

Wetzel et al. (1981)
5M, 6F (29.7)

placebo controlled

crossover

32M (34.5)

placebo controlled between groups

$6 \mathrm{M}, 6 \mathrm{~F}$

placebo controlled

crossover

12M, 11F (32)

placebo controlled

crossover

6M, 6F (27.5)
$10 / 20 / 40 \mathrm{mg}$

Arithmetic problems

CPT Double (omission errors)

CPT Double (commission errors)

CPT Double (sensitivity)

CPT Double (RT)

PAL (CVC pairs)

Serial visual WM task

N-back task (RT)

$\mathrm{N}$-back task (accuracy)

visual attention task

WLT

numerical problems

RPS

WM sig

WM ns

A/V ns

VEM ns

$20 \mathrm{mg}$

$0.5 \mathrm{mg} / \mathrm{kg}$

PAL (word pairs)

VEM sig (decreased performance) 


\begin{tabular}{|c|c|c|c|c|c|}
\hline Study & $\begin{array}{l}\text { Nr. of pp M, F (age) } \\
\text { design }\end{array}$ & Dose & Task/measure & Domain & Effect \\
\hline $\exp 1$ & placebo controlled & & picture recognition & VSM & ns \\
\hline [45] & crossover & & Story recall & VEM & $\begin{array}{l}\text { sig (decreased } \\
\text { perforance) }\end{array}$ \\
\hline Wetzel et al. (1981) & $6 \mathrm{M}, 6 \mathrm{~F}(26.6)$ & $0.1 / 0.25 \mathrm{mg} / \mathrm{kg}$ & PAL (word pairs) & VEM & ns \\
\hline $\exp 2$ & placebo controlled & & Picture recognition & VSM & ns \\
\hline [45] & crossover & & Story recall & VEM & ns \\
\hline
\end{tabular}

(1) Participants received $15 \mathrm{mg}$ MPH unless high or low in body weight in which case they received 20 or $10 \mathrm{mg}$ respectively

(2) MPH lessens effect of emotionally arousing material on memory, higher performance on neutral material compared to placebo

(3) Enhanced performance when MPH was taken on the first session

(4) MPH causing impairment when taken on second session

(5) Enhanced performance when MPH was taken on the first session, but impaired when taken on second session

Table 3

Percentages of studies showing cognition enhancing effects of methylphenidate in each of six cognitive domains (Total) and per dose level (Low, Medium, High). Study reference numbers refer to studies described in Table 2

\begin{tabular}{|c|c|c|c|c|c|c|}
\hline & Total & Low & Medium & High & $\begin{array}{r}\text { nr. of measures } \\
\text { (nr. of studies) }\end{array}$ & Study references \\
\hline Working memory & 68 & 0 & 65 & 65 & $15(12)$ & $1,7,13,17,19,20,27,30,31,33,41,42$ \\
\hline Speed of processing & 61 & 79 & 57 & 47 & $20(12)$ & $9,14,17,18,20,21,24,27,29,32,36,37$ \\
\hline Verbal learning and memory & 34 & 64 & 64 & 25 & $18(11)$ & $6,7,10,21,23,26,27,30,40,43,45$ \\
\hline Reasoning and Problem solving & 18 & 1 & 18 & 74 & $10(6)$ & $17,2130,38,39,44$ \\
\hline Attention and vigilance & 16 & 41 & 32 & -3 & $71(20)$ & $\begin{array}{l}1,2,3,4,10,11,12,14,15,17,20,21,22, \\
25,30,33,34,35,40,42\end{array}$ \\
\hline Visual learning and memory & 0 & 0 & 0 & 0 & $8(6)$ & $5,13,16,18,28,45$ \\
\hline
\end{tabular}


of) keys in response to certain stimuli showed improved performance after $\mathrm{MPH}$, including the SERS task, the circular lights task, two Contingent Negative Variation (CNV) tasks and a motor task (Callaway, 1984; Halliday, et al., 1986; Kollins, Rush, Pazzaglia, \& Ali, 1998; Linssen, et al., 2011; Naylor, Halliday, \& Callaway, 1985). DSST was employed in four studies, none of which found effects of MPH (Kollins, et al., 1998; Kuypers \& Ramaekers, 2005; Rush, Essman, Simpson, \& Baker, 2001; Rush, Kollins, \& Pazzaglia, 1998). One study employed a verbal fluency test, requiring participants to generate as many words as possible within one minute starting with a certain letter or from a certain semantic category, and found no MPH effect (Elliott, et al., 1997), while another study observed that participants could process more letters (that were presented on a computer screen and which the participants were asked to report after the trial) per second after $40 \mathrm{mg}$ of MPH (Finke, et al., 2010). The TMT was left unaffected by MPH (Hermens, et al., 2007).

\section{Reasoning and problem solving}

The next cognitive domain involves planning and decision making, aspects of cognition that are usually referred to as executive functioning. However, in order to allow a separate category on working memory, which is often included in executive functioning, this domain is named reasoning and problem solving. A typical task in this domain is the Tower of London, in which balls have to be arranged on pegs according to a predefined pattern in as little steps as possible. Performance on this task was observed to decrease with MPH when the drug was administered on the second session in a crossover study (Elliott, et al., 1997). However, in a non-motor version of this task, in which participants are required to work out how many steps are needed without actually performing them, improved performance was observed when MPH was administered on the first session but performance was impaired by MPH when administered on the second session (Elliott, et al., 1997). It is important to note though that there were no statistically significant main effects across the sessions. In a similar (non-motor) task, some minor performance enhancing effects of $\mathrm{MPH}$ were observed, but these were more likely spurious findings (Linssen, et al., 2012).

The study previously referred to also administered a task in which participants are asked to touch four red squares on a computer screen in as many different sequences as possible (Elliott, et al., 1997). Performance on this task was improved by MPH. A variety of other tasks is classified as assessing reasoning and problem solving skills, for example arithmetic problem solving and maze learning. Two studies tested MPH effects on the ability to solve arithmetic problems (Stoops, Lile, Fillmore, Glaser, \& Rush, 2005; Volkow, et al., 2008). Improved performance was observed in one of 
the studies, but not in the other. Maze learning was not affected by MPH (Hermens, et al., 2007). A more complex task is the arcade like game called Telekinesis Star Wars, described by Schroeder et al. (1987). It assesses problem solving, using three variables that reflect performance and the extent to which high level strategies are developed. MPH did not affect hit rate and decreased the rate at which adaptive strategies are developed (Schroeder, Mann-Koepke, Gualtieri, Eckerman, \& Breese, 1987).

Overall, $18 \%$ of the results reflected improved performance within this domain after MPH. Based on the limited number of studies that assessed effects on reasoning and problem solving tasks, conclusions on the doseresponse relationship within this category will not be drawn.

\section{Attention/vigilance}

As William James already stated in 1890 , 'everyone knows what attention is' (James, 1890). It is, however, difficult to give a clear and inclusive definition. It involves filtering information, focusing on certain aspects of what is perceived, while disregarding others. Many different forms of attention are distinguished (e.g. sustained attention, focused attention, divided attention). In this review, however, a collective domain attention and vigilance is employed.

The continuous performance test (CPT) is one of the most applied and most sensitive tests to assess sustained attention and vigilance (Riccio, Waldrop, Reynolds, \& Lowe, 2001). CPT requires participants to respond to relatively infrequent target stimuli that are embedded in a continuous presentation of stimuli. Different variables reflecting task performance may be reported, including omission errors (failing to respond to target stimuli), commission errors (responding to non-target stimuli), response time and sensitivity (measuring the ability to discriminate target stimuli from nontarget stimuli). CPT was employed in $8 \mathrm{MPH}$ studies, six of which showed improved performance on at least one of the reported measures (Agay, et al., 2010; Aman, Vamos, \& Werry, 1984; Camp-Bruno \& Herting, 1994; Coons, et al., 1981; Cooper, et al., 2005; Halliday, et al., 1986; Hermens, et al., 2007; Strauss, et al., 1984). Hence, CPT results have quite a significant share in the $16 \%$ of measures that are improved by MPH. A task similar to the CPT is the rapid visual information processing task applied by Elliott et al. (1997) in which participants have to respond to three different pre-specified sequences of digits within a stream of rapid presentations of digits. In this study no effect of MPH on response accuracy was observed, but MPH did speed the responses. Besides the CPT and the Rapid visual information processing (RVIP), a wide variety of similar tasks exists, that all entail detection of a target stimulus or event, and are used to assess sustained attention or 
vigilance. These include for example oddball tasks and the Mackworth clock task. The majority of these tasks were not affected by MPH (Anderer, Saletu, Semlitsch, \& Pascual-Marqui, 2002; Bishop, Roehrs, Rosenthal, \& Roth, 1997; Coons, et al., 1981; Hink, Fenton, Tinklenberg, Pfefferbaum, \& Kopell, 1978; Oken, et al., 1995; Roehrs, Papineau, Rosenthal, \& Roth, 1999; Tomasi, et al., 2011), but some showed improved performance after MPH (Hermens, et al., 2007). MPH speeded responses on the Paced auditory serial addition test, which is also considered a measure of sustained attention (Hermens, et al., 2007).

In another well known type of tasks, the Go/No-go tasks, stimulus response contingencies are exactly opposite to those in target detection tasks. That is, instead of only responding in target trials, Go/No-go tasks require participants to respond in the majority of trials (Go) but withhold their response to certain stimuli (No-go). One study reported to use this task, observing speeded response times but no effect on hit rate or impulsivity errors (Kratz, et al., 2009). Performance of the stop signal task, in which participants have to inhibit an initiated Go response was observed to improve on MPH (Linssen, et al., 2012).

Set shifting tasks require flexibility and are sometimes classified as divided attention tasks. In one example of such a test participants have to switch between attending to visual and auditory stimuli, responding only to target stimuli. MPH significantly enhanced performance on this task (Linssen, et al., 2012). A slightly more complex task is the intra-/extradimensional (ID/ED) shift task which requires participants to make visual discriminations while stimulus characteristics vary along different dimensions and the relevant dimension reverses between different task stages. Of the two studies employing this task, one found no effect of MPH whereas another study observed decreased performance on this task after MPH (Elliott, et al., 1997; Rogers, et al., 1999).

Other divided attention tasks have been used in several studies. A typical task requires participants to track a moving target with a joystick while simultaneously responding to the appearance of white circle. Using this task, one study found no effect of MPH (Bishop, et al., 1997), whereas another observed speeded response times to the white circle when it was presented in the center of the screen (Roehrs, et al., 1999). A task requiring both divided as well as focused attention is the dichotic monitoring task. Most variables measured in this task were not affected by $\mathrm{MPH}$, but the drug did reduce the error rate in one of the two studies (Clark, Geffen, \& Geffen, 1986a, 1986b). MPH also improved performance on the covert orienting of spatial attention task (Oken, et al., 1995). 


\section{Visual learning and memory}

The last cognitive domain to discuss is visual learning and memory. Tasks classified as assessing performance in this cognitive domain are for example picture recognition tests and other learning and memory tasks involving visual stimuli. None of the studies found an effect of MPH on visual learning and memory (Brignell \& Curran, 2006; Bullmore, et al., 2003; Clatworthy, et al., 2009; C. M. Dodds, et al., 2008; Finke, et al., 2010; Kuypers \& Ramaekers, 2007; Wetzel, et al., 1981).

\section{Conclusions}

In this review we present how effectively MPH affects performance in several cognitive domains. The extent to which MPH enhances cognitive performance was quantified by giving a percentage (weighed on the basis of specified criteria such as number of subjects) that reflects the number of studies showing positive effects of $\mathrm{MPH}$. This is a major improvement compared to earlier reviews on the effect of MPH on cognition in which: only some domains of cognition were considered; the categorization in domains was less well specified (Repantis, et al., 2010; M. E. Smith \& Farah, 2011); and/or the quality was either not taken into account or based on criteria that hardly differentiate between studies (e.g. double blind design, randomization (Jadad, et al., 1996)). Furthermore, it must be noted that this review compares 45 studies, which is considerably more than was done in previous reviews. The studies reviewed here show that MPH improves cognitive performance in the healthy population in the domains of working memory and speed of processing, and to a lesser extent may also improve verbal learning and memory, reasoning and problem solving and attention and vigilance but does not have an effect on visual learning and memory (see Table 3).

There were quite large differences between the domains with respect to the cognition enhancing effect of $\mathrm{MPH}$, the lowest percentage being 0 and the highest almost $70 \%$. These differences confirm that in studying cognitive performance it is important to distinguish between different domains of cognitive function. MPH may not globally improve cognitive performance, but it has potential to enhance certain aspects of cognitive performance at certain doses.

Considering the known involvement of dopamine in working memory, $\mathrm{MPH}$ may be expected to improve working memory. Indeed, working memory was the most affected cognitive domain. The proportion of the effect of MPH was similar across different types of tasks and medium and high doses. However, in the spatial working memory tests, it is possible that the MPHeffect follows an inverted $U$ shaped curve as a function of dose, since a high 
dose $(60 \mathrm{mg})$ exerted no effect on a spatial working memory task that was affected by lower doses in other studies ((Clatworthy, et al., 2009; Elliott, et al., 1997; Mehta, et al., 2000), but note that Elliott et al. (1997) found no main effects).

The second most affected domain was speed of processing. Most of the affected tasks involve simple motor responses, whereas the tasks that are not affected are generally slightly more difficult. Another striking observation is that the low dose exerts the highest proportion of effects on cognitive performance in this domain, followed by the medium dose and the highest dose showing the lowest proportion of effects. This suggests that within this domain the optimal dose is rather low and the higher the dose, the less healthy volunteers benefit from it.

Declarative memory is not typically associated with dopaminergic activity. It is therefore not surprising that the domain of verbal learning and memory is less affected by MPH compared to the first two domains. Positive effects of MPH on declarative memory fit well with accounts of related stimulant drugs affecting word list learning (Soetens, Casaer, D'Hooge, \& Hueting, 1995; Soetens, D'Hooge, \& Hueting, 1993; Zeeuws \& Soetens, 2007). The relationship between MPH dose and its effect on word list learning seems to be a positive and linear one. This contrasts with the striking finding that a high MPH dose can decrease performance on a PAL task and story recall. Across the domain of verbal learning and memory the low and medium doses exert more cognition enhancing effects than the high dose. Hence, even within one domain, different dose-response relationship may be observed for different tasks.

The domains of reasoning and problem solving and attention and vigilance were virtually equally affected by $\mathrm{MPH}$. Within the domain of reasoning and problem solving, MPH seems to mainly affect the Tower of London task. However the small amount of measures reported within in this domain restrict the conclusions regarding the effects of $\mathrm{MPH}$ on specific tasks or dose related effects.

Based on previous literature, associating attention with dopaminergic activity (Cools \& Robbins, 2004; Nieoullon, 2002; Nieoullon \& Coquerel, 2003) it was expected that attention enhancing effects of MPH would be revealed in this review. However, only a relatively small proportion of studies showed that attention was affected by MPH. With 71 measures reported in this domain, the conclusion that MPH has little effect on attention and vigilance in the healthy population should be a reliable one.

Visual learning and memory remained unaffected by MPH in all studies listed here. It is, however, important to note that the data reported within this domain only comprises 8 measures. This gives the conclusion regarding 
the absence of an MPH effect on visual learning and memory somewhat less weight.

The dose response relationship of the cognition modulating effect of MPH differs across different cognitive domains. Performance on working memory tasks is most effectively enhanced by medium and high doses. On the other hand, in the domains of speed of processing, verbal learning and memory and attention and vigilance, lower doses are more effective. An explanation for this may be that in healthy volunteers, the dopamine availability is already close to the optimal level. Enhancing dopamine activity may push the level of dopamine beyond the optimum without improving performance or even leading to suboptimal performance.

Factors limiting the conclusions in this review relate to the decisions that were made on how to categorize, list and present the data. As there were quite some tasks that were not described in the papers on which the categorization in dimensions was based, the authors had to assign those to the different domains. Although this was done very carefully, the choices may sometimes be arbitrary. Other decisions that were well thought through, but might still be somewhat arbitrary include: (1) some tasks were split into several measures whereas others were not; (2) a statistical trend was given a weight of 0.5 ; (3) if it was not clear which of the reported doses led to significant effects, it was assumed that the reported effect was applicable to every dose; (4) sometimes two low doses are reported, but they are only weighed once; (5) there was no correction for multiple comparisons for multiple dosing. Finally a major limitation of any review is that studies that did not show significant effects are generally underreported (i.e. publication bias). Therefore, any review is likely to present an overestimation of the reported effects.

In sum, the studies reviewed here showed improvement of working memory and speed of processing and, to a lesser extent of verbal learning and memory, reasoning and problem solving and attention and vigilance. As detailed above, the effects are not such that taking MPH guarantees better cognitive function. In fact, performance could even decrease after MPH intake. MPH effects are dose dependent and the dose-response relationship differs between the different cognitive domains. For speed of processing, verbal learning and memory and attention and vigilance there is a negative dose-response relationship whereas the results within the working memory domain are consistent with the inverted-U curve. It is important to note that this review only included studies on acute dose effects and we cannot comment on effects of long-term MPH use. In conclusion, healthy individuals should be cautious when considering using MPH. The actual effects may be smaller than expected by abusers 
Chapter 2. Methylphenidate produces selective enhancement of declarative memory consolidation in healthy volunteers

Linssen, A. M., Vuurman, E. F., Sambeth, A., \& Riedel, W. J. (2012)

Psychopharmacology, 221(4), 611-619

\section{Abstract}

Rationale: Methylphenidate inhibits the reuptake of dopamine and noradrenaline and is used to treat children with attention deficit hyperactivity disorder (ADHD). Besides reducing behavioral symptoms, it improves their cognitive function. There are also observations of methylphenidate-induced cognition enhancement in healthy adults, although studies in this area are relatively sparse. We assessed the possible memoryenhancing properties of methylphenidate.

Objective: In the current study, the possible enhancing effects of three doses of methylphenidate on declarative and working memory, attention, response inhibition and planning were investigated in healthy volunteers.

Methods: In a double blind placebo-controlled crossover study, 19 healthy young male volunteers were tested after a single dose of placebo or 10, 20 or $40 \mathrm{mg}$ of methylphenidate. Cognitive performance testing included a word learning test as a measure of declarative memory, a spatial working memory test, a set-shifting test, a stop signal test and a computerized version of the Tower of London planning test.

Results: Declarative memory consolidation was significantly improved relative to placebo after 20 and $40 \mathrm{mg}$ of methylphenidate. Methylphenidate also improved set shifting and stopped signal task performance but did not affect spatial working memory or planning.

Conclusions: To the best of our knowledge, this is the first study reporting enhanced declarative memory consolidation after methylphenidate in a doserelated fashion over a dose range that is presumed to reflect a wide range of dopamine reuptake inhibition. 


\section{Introduction}

Cognition enhancement has received much attention in recent scientific literature, due to our aging society and the increasing prevalence of Alzheimer's Disease. However, the healthy young population also engages in drug use to enhance cognition as is illustrated by the abundant illicit use of drugs that are normally prescribed for the treatment of attention deficit hyperactivity disorder (ADHD) including methylphenidate (Ritalin) and other amphetaminelike stimulants, which are believed to improve cognitive performance (Greely, et al., 2008; Maher, 2008). This has raised concern regarding the ethical and safety aspects of potential cognition-enhancing drugs (Greely, et al., 2008; Larriviere, et al., 2009; Sahakian \& Morein-Zamir, 2007). These issues aside, it is important to know if these drugs do actually have cognition-enhancing effects in healthy subjects.

Methylphenidate inhibits the reuptake of the catecholamines dopamine and noradrenaline by blocking the transporters and thus enhances catecholamine availability (Hannestad, et al., 2010; Volkow, Wang, Fowler, Gatley, et al., 1998). Methylphenidate treatment does not only reduce behavioural symptoms in children with ADHD but also improves their cognitive function (Pietrzak, et al., 2006). There are also observations of methylphenidate induced cognition enhancement in healthy adults (Elliott, et al., 1997; Mehta, et al., 2000), although studies in this area are relatively sparse.

Previous studies on the effects of methylphenidate on cognitive function in healthy volunteers have mainly focused on cognitive domains which are known to be affected in ADHD or to involve dopaminergic action. These include attention, response inhibition, planning and (working) memory (Cools \& Robbins, 2004; Luciana \& Collins, 1997; Luciana, Depue, Arbisi, \& Leon, 1992; Mehta, Sahakian, McKenna, \& Robbins, 1999; Pietrzak, et al., 2006). One aspect of attention studied in healthy volunteers is divided attention. Methylphenidate has been observed to improve performance on a divided attention task, in which participants have to track a moving target with a joystick and simultaneously press a button in response to the appearance of a visual stimulus (Bishop, et al., 1997; Roehrs, et al., 1999). These effects were most prominent when participants were sleep-deprived.

Attentional control has been assessed in healthy volunteers using an intradimensional/extradimensional shift task, which requires participants to make visual discriminations while stimulus characteristics vary along different dimensions and the relevant dimension reverses between different task stages (Rogers, et al., 1999). Although Elliott et al. (1997) found no effect of methylphenidate on this task, Rogers et al. (1999) observed an enhanced 
ability to reallocate attention towards newly relevant features of environmental stimuli after methylphenidate.

Response inhibition, as assessed in a Go-NoGo task has been shown to improve with methylphenidate in older adults (Ben-Itzhak, Giladi, Gruendlinger, \& Hausdorff, 2008). Planning is also observed to be affected by methylphenidate in healthy adults. In two versions of the Tower of London task, enhanced performance was observed when methylphenidate was administered in the first of two sessions as opposed to impaired performance when the drug was taken in the second session (and placebo in the first, (Elliott, et al., 1997)). Elliott et al. explain this result hypothesizing that methylphenidate enhances performance on novel tasks but impairs performance on familiar tasks.

There are several reports of improved performance on both nonspatial and spatial working memory tasks after methylphenidate administration in healthy adults. For example, methylphenidate enhances performance on the Sternberg memory scanning task, in which participants are asked to judge whether probe letters were in the memory set that was learned at the beginning of the task (Brumaghim \& Klorman, 1998; Fitzpatrick, et al., 1988). Improvement of spatial working memory has been observed within a selfordered search task in which participants search for tokens in an array of boxes displayed on a computer screen (Elliott, et al., 1997; Mehta, et al., 2000).

Besides the domains described above, few researchers have studied the influence of methylphenidate on immediate and delayed recall of word lists (Bray, et al., 2004; Camp-Bruno \& Herting, 1994; Hermens, et al., 2007; Kuypers \& Ramaekers, 2005), none of whom found a significant effect. This is surprising as methylphenidate is known to enhance delayed recall of word lists in ADHD (Evans, Gualtieri, \& Amara, 1986). The pharmacologically similar compound amphetamine has shown to improve consolidation and retention of word lists while leaving acquisition unaffected (Advokat, 2010). Experiments from Soetens and Zeeuws (Soetens, et al., 1995; Soetens, et al., 1993; Zeeuws \& Soetens, 2007) have shown that amphetamine facilitates verbal memory recall if it is active in the brain during the consolidation phase (Soetens, et al., 1995). Additional experiments showed that the effect increases with longer presentation time of the words, lasts at least three days, is independent of retrieval and generalizes to recognition (Soetens, et al., 1995; Zeeuws, Deroost, \& Soetens, 2010; Zeeuws \& Soetens, 2007).

Importantly, task procedures used by Soetens and Zeeuws were rather different from those used in the methylphenidate studies cited above. Hermens et al., Kuypers \& Ramaekers and Bray et al. used word lists containing only 12 or 15 items. In combination with repeated presentations 
(three or four times), this may have led to a ceiling effect in performance. In line with this notion, using lists of 24 easy nouns that were presented only once, Camp-Bruno \& Herting (1994) did observe improved performance in both immediate and delayed recall, although this effect was not significant.

The aim of the current study was to find out whether enhanced word recall could be observed after methylphenidate with a more optimal experimental design. In order to avoid possible ceiling effects suspected to have influenced the studies mentioned above, a 30-word learning test was used in the current study. Performance on the 30 word lists of the visual verbal learning task was tested in 19 healthy volunteers after 10, 20 or $40 \mathrm{mg}$ of methylphenidate and placebo. To get a more complete view of methylphenidate's cognitive effects, not only extending previous findings but also putting current effects of methylphenidate on word learning into perspective, additional cognitive tasks were administered. These additional tasks were carefully selected to represent the most relevant cognitive domains, both with respect to the most common clinical application of methylphenidate (i.e. treatment of $A D H D$ ) and dopaminergic involvement. Hence, we included tasks assessing attention, response inhibition, planning and working memory.

The design of the current study, using three different doses of methylphenidate in a placebo-controlled within-subjects experiment allows examination of a dose-response relationship for this drug and its effect on cognition. It addresses some methodological issues observed in other studies using only a single dose or comparing different doses in parallel groups (Elliott, et al., 1997; Mehta, et al., 2000). Dose related effects of moderate to high doses of methylphenidate on memory consolidation were expected to be reflected by better delayed recall. Performance on the other tasks was also expected to improve after moderate to high doses of methylphenidate. Based on the effect of methylphenidate on psychomotor function it was expected that improved performance on these tasks may mainly be reflected by faster response times.

\section{Method}

\section{Participants}

Nineteen healthy male volunteers (mean age $=23.4, \mathrm{SD}=5.4$, range $=19-37$ ) were selected and paid to participate. Participants were recruited by means of local advertisements. Prescreening occurred using a medical history questionnaire and was followed by medical examination.

The main inclusion criteria were: between 18 and 40 years of age, body mass index between 18 and $30 \mathrm{~kg} / \mathrm{m}^{2}$ and right-handedness. The main 
exclusion criteria were history or presence of mental or physical disorders consumption of more than 21 alcohol units per week or more than five caffeine containing drinks per day. In addition, volunteers with polymorphisms of the CES1 gene indicative of being a poor metabolizer of methylphenidate were excluded. One participant was excluded based on this criterion.

All subjects gave written informed consent. The study was carried out in accordance with the Declaration of Helsinki and approved by the medical ethical committee of Maastricht University.

\section{Design}

The study was conducted according to a double-blind, placebo-controlled, four-way crossover design. Between the testing days a period of at least $48 \mathrm{~h}$ elapsed, but generally testing days were scheduled approximately 1 week apart. Each participant received one of four single treatments including placebo (PLA) and 10, 20 and $40 \mathrm{mg}$ methylphenidate (M10, M20 and M40) on each testing day. The order of the treatments was randomized following a Williams Latin Square design resulting in four different sequences.

\section{Visual verbal learning test}

This task is an adapted version of Rey's Auditory Verbal Learning Test (Lezak, 1995). The VVLT was used to measure declarative memory. It included 30 words that were presented on a computer screen (stimulus presentation time and inter-stimulus interval were $1000 \mathrm{~ms}$, (Klaassen, Riedel, Deutz, \& Van Praag, 2002)). This presentation was repeated three times using the same sequence of words, each time followed by immediate free recall of all remembered words. Thirty minutes after immediate free recall of the final series participants were subjected to a delayed recall test and a recognition test. During the latter, 30 words were presented, 15 of which were previously presented and another 15 that were new. Outcome measures of the immediate and delayed recall tests were total number of correctly recalled words during immediate recall (summed over three trials) and number of correctly recalled words during delayed recall. The dependent measures of the recognition test were median reaction time, measured in milliseconds and sensitivity $\left(A^{\prime}\right)$. $A^{\prime}$ is calculated as follows: $A^{\prime}=1-1 / 4[f r / c r+(1-c r) /(1-$ $\mathrm{fr})$ ], with $\mathrm{fr}=$ falsely recognized words and $\mathrm{cr}=$ correctly recognized words.

\section{Spatial working memory task}

The 'Object relocation' program was used as a spatial working memory task (Kessels, Postma, \& de Haan, 1999; Kessels, Postma, Wester, \& de Haan, 2000). In this task participants were required to relocate ten visual stimuli (small drawings of objects such as a car or a flower) that were presented on a 
computer screen in a $15-\mathrm{cm}^{2}$ sized square to their original location. The task consisted of two parts. In the first part, called 'object to position' or OTP, participants had $15 \mathrm{~s}$ time to memorize the locations of the objects and were asked to drag the objects to the correct positions, which were cued with black dots. In the second part, the combined (COM) part, participants had $30 \mathrm{~s}$ to memorize the locations and this time, there were no cues to indicate the previous positions. Participants were instructed to place the objects in their original position as accurately as possible. In both parts, there was no time limit for the relocation of the stimuli. The dependent variables were percentage correct for OTP and percentage correct, absolute error and positional fit for COM. In the COM part, percentage correct was calculated such that relocation would be correct when the object was placed within 20 $\mathrm{mm}$ from the original location. Absolute error was the absolute distance in millimeters an object was relocated away from its original position. Positional fit measured the ability to remember the locations per se, independent from the ability to remember which object was in each location and computed as the best fit score yielding the smallest distance error for the stimulus as a whole.

\section{Set shifting task}

The set shifting task used in this study combined the methods of Müller and Townsend (J. Muller, et al., 2007; Townsend, Adamo, \& Haist, 2006). The task measured cognitive flexibility and the influence of reward. Participants were presented with a stream of auditory and visual stimuli, including light and dark blue squares on a computer screen and high and low tones through headphones (stimulus presentation time was $150 \mathrm{~ms}$ and inter-stimulus interval was $850 \mathrm{~ms}$ ). Assignments so as to respond to the tones ('Hear trials') or the squares ('Look' trials) were interspersed with the stimuli and presented for $1.000 \mathrm{~ms}$. Participants were told to respond only to the dark blue square and the high tones, while ignoring the light blue squares and low tones. In half of the trials, a ' $€$ ' sign on the computer screen indicated that if they would perform very well (correct and fast responses), they would earn an additional monetary reward (awarded to the best performer in the study). The experiment contained six blocks, of which three were 'reward blocks' and three were 'nonreward blocks'. Each block contained 56 stimuli and switching occurred after a variable number of trials, ranging between five and nine trials. The sequence of blocks was randomized between participants and sessions. After a block containing the reward stimuli, the participants received feedback on how well they performed. Participants were expected to respond faster in the reward than in the control conditions. Dependent measures were response times in correct trials and percentage of correct 
responses. These were calculated separately for the four different trial types: Look in the reward blocks; Look in the non-reward blocks; Hear in the reward blocks; and Hear in the nonreward blocks.

\section{Stop signal task}

The stop signal task was used to measure motor impulsivity and adapted from that used by Fillmore et al. (Fillmore \& Rush, 2002). In this task participants were required to respond to go signals and inhibit the response when a stop signal was presented. On a computer screen, the go signals were visually presented letters, A, B, C and D (stimuli were presented for $500 \mathrm{~ms}$, or until the participant responded; interstimulus interval was $1500 \mathrm{~ms}$ ). The stop signal was the visual presentation of $a$ '*'at one of the corners of the screen. Participants were instructed to respond as fast as possible with left (to $A$ and $C$ ) and right (to $B$ and $D$ ) index fingers. Stop signals were presented at delays of 50, 150, 250, and $350 \mathrm{~ms}$. A single test consisted of 176 trials in which each of the four-letter stimuli was presented equally often. A stop signal occurred in 48 trials during a test. Dependent variables were the proportion of commission errors on stop trials and the reaction times on go and stop-signal trials (i.e. stop reaction time). Reaction time to stop-signal trials represented the estimated mean time required to inhibit a response. The method for calculating stop reaction time was taken from the race model of inhibitory control (Logan, 1994). Stop reaction time was calculated by subtracting the stop-signal delay from the reaction time on go-trials associated with the $n$th percentile of the reaction time distribution. The $n$th percentile corresponds to the percentage of commission errors (Logan, 1994; Ramaekers, et al., In press).

\section{Tower of London}

Planning ability was assessed by a computerized version of the Tower of London task (Sobczak, et al., 2002). On a computer screen, two arrays of differently colored balls (red, yellow and blue) on sticks were presented until the subject responded. The subject was requested to indicate the minimal number of steps necessary to rearrange the balls on the lower configuration to match the arrangement presented on the top half of the screen. The subjects had to count the number of moves and then respond by pressing the appropriate response button (two to six steps) as quickly as possible. The complexity of the task was dependent on the minimal number of steps in which the rearrangement could be achieved: two, three, four and five steps and sometimes six steps, to avoid guessing (only two to five are analyzed). Performance was indicated by the slope coefficient of the linear regression of the median response time as a function of the number of steps. In addition, 
percentage correct and response time calculated per number of minimally required steps were taken into account.

\section{Procedure}

The current study was integrated in a larger study on the effects of methylphenidate on the Contingent Negative Variation (Linssen, et al., 2011). A training session in which the tasks were practiced took place within 2 weeks before the first testing day. Participants abstained from alcohol during the last $24 \mathrm{~h}$ prior to each testing day. On testing days, they were collected at their home and arrived at the lab in fasted condition at either 8.00 am or 8:30 am. Inclusion and exclusion criteria were checked. Next, participants were given a standardized meal for breakfast at 80 min pre dose $(t=-80)$. At $t=-$ 30, stop signal, set shifting and spatial working memory tests were administered to get a baseline measurement. At $t=0$ participants ingested four capsules with water, which contained either PLA, M10, M20 or M40. The stop signal, set shifting and spatial working memory tests were administered again at $\mathrm{t}=90$ and $\mathrm{t}=270$. The verbal word learning test and the Tower of London were administered at $t=150$, followed by delayed recall and recognition testing of the words approximately 30 min later. At the end of the testing day participants were returned to their home.

\section{Data analysis}

Data were analyzed using a repeated measures analysis of variance. Because one subject dropped out, there was a minimal deviation from complete balancing of treatment orders (order 1=PLA, M10, M20, M40 (five participants), order $2=M 10, M 40$, PLA, $M 20$ (five participants), order $3=M 20$, PLA, M40, M10 (four participants) and order $4=$ M40, M20, M10, PLA (five participants)). For this reason, treatment order was entered as a between subjects factor. Since treatment consisted of different doses of the same drug, main effects of the drug were not evaluated. Instead, a priori planned comparisons between each dose relative to placebo were tested for significance, at $\mathrm{p}<.05$, using LSD correction.

\section{Results}

\section{Visual verbal learning test}

Mean values of dependent measures of the visual verbal learning task are presented in Figure 1. Methylphenidate had no effect on immediate recall. However, delayed recall improved after $M 20\left(F_{1,14}=4.937, p<.05\right)$ and $M 40$ $\left(\mathrm{F}_{1,14}=6.084, \mathrm{p}<.03\right)$ compared to placebo. 
Median reaction time on the recognition test was slower after $M 40$ $\left(F_{1,14}=5.953, p<.05\right)$. Sensitivity was higher after $40 \mathrm{mg}$ of methylphenidate $\left(F_{1,14}=4.878, p<.05\right)$.
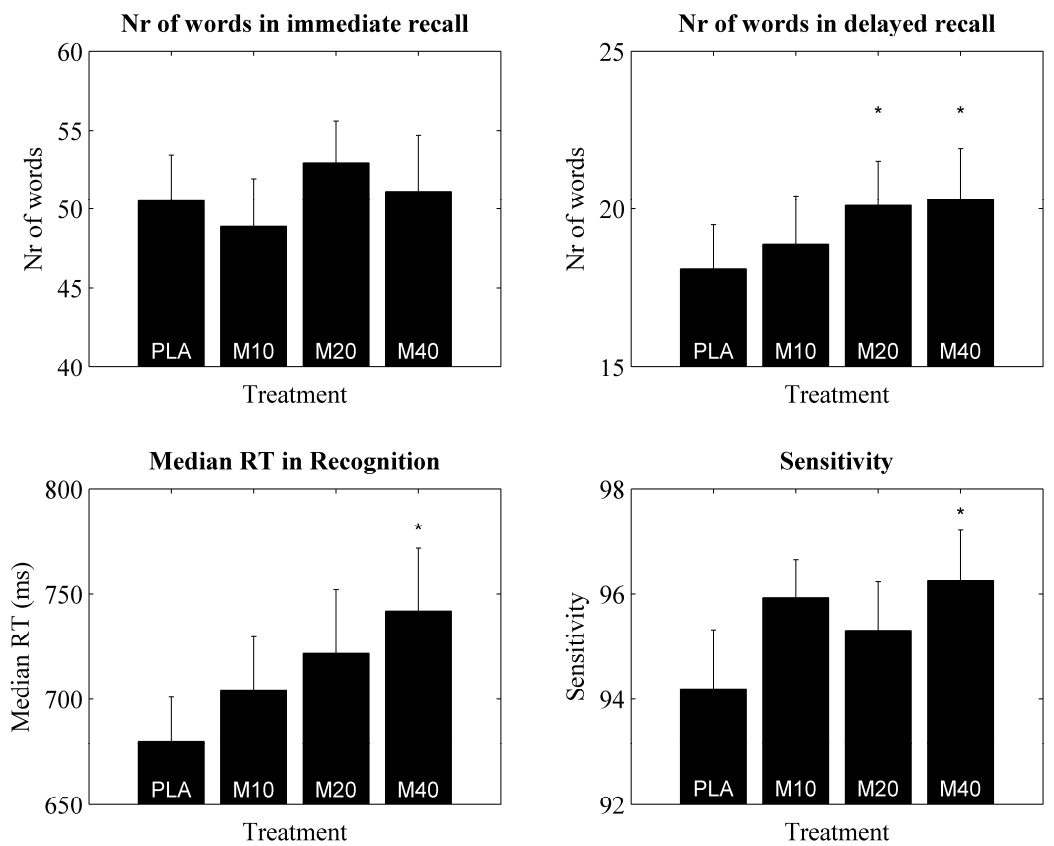

Figure 1. Means of dependent measures (a: Total number of correctly recalled words in immediate recall; b: Number of words recalled in delayed recall; c: Median response time in recognition test; d: Sensitivity of recognition test) of the word learning test after placebo and methylphenidate, 10, 20 and $40 \mathrm{mg}$ (PLA, M10, M20 and M40 respectively). Significant differences relative to placebo are indicated by *.

\section{Spatial working memory task}

Methylphenidate did not significantly affect spatial working memory task performance (Table 1).

\section{Set shifting task}

Methylphenidate did not affect response time and percentage correct in the 'look' trials in the reward blocks. In the nonreward blocks there was also no effect of the drug on percentage correct. However, methylphenidate led to faster responses on the 'look' trials of the nonreward blocks after M40 at T90 $\left(F_{1,15}=9.589, p<.01\right)$ and after $M 10$ and $M 40$ at T270 (M10: $F_{1,14}=5.363, p<.04$, M40: $\left.F_{1,14}=14.145, p<.01\right)$ 
Responses to 'hear' trials were faster in the non-reward parts of the

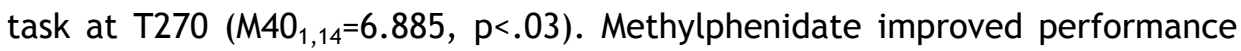
in the hear trials in both reward $\left(M 40: F_{1,15}=10.261, p<.01\right)$ and non-reward trials (M20: $\left.F_{1,15}=4.713, p<.05, M 40: F_{1,15}=5.809, p<.03\right)$ at T90 and reward trials at T270 (M40: $\left.F_{1,14}=6.764, p<.03\right)$.

\section{Stop signal task}

A dose of $20 \mathrm{mg}$ of methylphenidate led to faster go and stop responses at T270 (go: $\mathrm{F}_{1,14}=5.479, \mathrm{p}<.04$; stop: $\mathrm{F}_{1,14}=9.341, \mathrm{p}<.01$ ). Performance on this task, as measured by percentage correct was improved after $M 40$ in stop trials at T90 $\left(\mathrm{F}_{1,15}=10.698, \mathrm{p}<.01\right)$.

\section{T ower of London}

Methylphenidate only positively affected performance of trials that could be solved in three steps, when given at a dose of $20 \mathrm{mg}\left(\mathrm{F}_{1,15}=5.065, \mathrm{p}<.05\right)$. Participants responded faster after $\mathrm{M} 10$ and in the trials that could be solved in five steps (M10: $\left.F_{1,15}=7.616, p<.02\right)$ (Table 2 ).

\section{Discussion}

In this dose-response study, different doses of methylphenidate were tested for their effect on memory and other cognitive functions in healthy volunteers. Methylphenidate improved delayed recall of word lists. In addition, set shifting and stop signal test performance improved after methylphenidate. Methylphenidate had little effect on the Tower of London task performance and no effect on performance of the spatial working memory task.

Based on earlier findings of enhanced declarative memory consolidation after amphetamine, it was expected that methylphenidate would improve performance on the 30-word learning test. Results of the current study confirmed this expectation. Participants recalled more words in the delayed recall test after 20 and $40 \mathrm{mg}$ of methylphenidate, compared to placebo. Since no improvement was observed at immediate recall, enhanced delayed recall is likely caused by better consolidation. To the best of our knowledge we are the first to report better consolidation of word lists after methylphenidate in healthy adults. Since previous studies used similar doses (20 or $45 \mathrm{mg}$ ), suboptimal dosing is an unlikely explanation for the lack of performance-enhancing effects in those studies. An important factor may be word list length. When testing memory in healthy volunteers, a ceiling effect is easily reached if relatively short lists are presented repeatedly. Since it was hypothesized that enhanced dopamine activity induced by methylphenidate 
Table 1

Mean values (SEM) of dependent measures of the spatial working memory test (SWM), Set shifting (SSHT) and stop signal (SST) after placebo and methylphenidate 10, 20 and $40 \mathrm{mg}$ (PLA, M10, M20 and M40 respectively). (OTP=object to position; COM=combined; abs err=absolute error; pos fit=positional fit; Look $€=$ Look reward trials; Look=Look non-reward trials; Hear $€=$ Hear reward trials; Hear=Hear non-reward trials; $R T=$ response time)

\begin{tabular}{|c|c|c|c|c|c|c|c|c|c|c|c|c|c|c|c|c|c|}
\hline & & T60 & & & & & & & & T240 & & & & & & & \\
\hline & & PLA & & M10 & & M20 & & M40 & & PLA & & M10 & & M20 & & M40 & \\
\hline \multicolumn{18}{|l|}{ SWM } \\
\hline OTP & $\%$ correct & 5.6 & $(4.5)$ & $-9.6^{\wedge}$ & (5.5) & -5.3 & $(-5.5)$ & -6.9 & $(4.9)$ & 2.4 & $(5.0)$ & -5.3 & $(6.0)$ & -4.9 & $(6.0)$ & -1.0 & $(4.0)$ \\
\hline \multirow[t]{3}{*}{ COM } & \% correct & -7.5 & $(6.0)$ & $9.9^{\wedge}$ & (6.4) & -10.0 & $(3.0)$ & -5.0 & (6.3) & -8.5 & $(4.4)$ & 3.4 & (6.9) & -11.9 & $(5.5)$ & 2.6 & $(6.8)$ \\
\hline & abs err & -17.6 & $(27.6)$ & -58.6 & (33.8) & 21.7 & $(25.4)$ & 8.6 & $(21.7)$ & 25.2 & (19.4) & 16.9 & $(23.3)$ & 25.6 & $(20.6)$ & 6.0 & $(28.9)$ \\
\hline & pos fit & 3.9 & (10.8) & -15.7 & (13.6) & 9.0 & $(8.4)$ & -0.0 & (11.9) & 22.4 & (10.9) & $-15.5^{\wedge}$ & $(12.5)$ & 16.1 & $(11.8)$ & 3.9 & $(13.0)$ \\
\hline \multicolumn{18}{|l|}{ SSHT } \\
\hline \multirow[t]{2}{*}{ Look $€$} & RT & -1.3 & (2.3) & $-1,6$ & $(-2.4)$ & $-1,8$ & $(-2.8)$ & $-2,9$ & $(2.0)$ & 1.6 & $(2.6)$ & -2.6 & (2.3) & -5.4 & $(2.3)$ & -4.8 & $(2.2)$ \\
\hline & \% correct & 0.2 & $(0.8)$ & 0.6 & $(1.0)$ & 1.0 & $(0.7)$ & 2.1 & $(1.8)$ & -0.4 & (1.2) & -0.7 & (1.3) & 0.6 & $(0.9)$ & 0.4 & (1.6) \\
\hline \multirow[t]{2}{*}{ Look } & RT & 0.7 & (1.9) & -3.3 & (1.8) & -0.9 & $(1.8)$ & $-6.9^{*}$ & $(1.8)$ & 3.0 & $(2.8)$ & $-3.9^{*}$ & (1.6) & $-4.0^{\wedge}$ & (1.6) & $-9.6^{*}$ & $(1.7)$ \\
\hline & $\%$ correct & 2.1 & (1.3) & 0.2 & $(0.5)$ & 0.5 & $(1.2)$ & 0.4 & $(0.6)$ & 1.0 & $(1.3)$ & 1.3 & $(0.5)$ & 0.6 & (1.1) & -0.1 & $(0.4)$ \\
\hline \multirow[t]{2}{*}{ Hear $€$} & $\mathrm{RT}$ & -8.5 & $(2.5)$ & -5.8 & $(2.3)$ & $2.5^{\wedge}$ & (4.1) & -3.8 & $(2.2)$ & -6.9 & (2.5) & -4.7 & (1.5) & -4.3 & $(3.0)$ & -4.2 & $(3.4)$ \\
\hline & $\%$ correct & -1.5 & $(0.9)$ & 0.3 & $(0.4)$ & $0.96^{\wedge}$ & $(1.0)$ & $2.3^{*}$ & $(0.9)$ & -1.0 & $(0.5)$ & 0.3 & $(0.6)$ & 1.0 & $(0.9)$ & $2.1^{*}$ & $(1.1)$ \\
\hline \multirow[t]{2}{*}{ Hear } & RT & 0.3 & $(2.6)$ & -5.1 & (2.5) & -1.5 & (2.5) & $-6.9^{\wedge}$ & $(2.0)$ & -0.5 & (1.7) & $-7.2^{\wedge}$ & $(2.3)$ & -5.9 & $(2.1)$ & $-8.7^{*}$ & $(2.6)$ \\
\hline & \% correct & -1.3 & $(0.5)$ & -1.3 & $(0.5)$ & $1.3^{*}$ & $(1.0)$ & $0.4^{*}$ & $(0.3)$ & -1.1 & $(0.9)$ & -0.7 & $(0.5)$ & 0.9 & $(0.6)$ & -0.1 & $(0.3)$ \\
\hline \multicolumn{18}{|l|}{ SST } \\
\hline \multirow[t]{2}{*}{ Go } & RT & -9.3 & (5.4) & -20.1 & $(6.8)$ & -20.0 & $(7.6)$ & $-33.6^{\wedge}$ & $(11.8)$ & 2.4 & $(7.4)$ & $-14.9^{\wedge}$ & (7.3) & $-20.7^{*}$ & (6.7) & $-24.6^{\wedge}$ & $(9.8)$ \\
\hline & $\%$ correct & 0.7 & $(0.7)$ & 0.4 & $(0.6)$ & -1.0 & $(1.2)$ & 0.5 & $(0.8)$ & 0.4 & $(0.7)$ & -0.8 & $(0.5)$ & -1.1 & $(1.0)$ & -1.3 & $(1.0)$ \\
\hline \multirow[t]{2}{*}{ Stop } & RT & -9.4 & $(8.8)$ & -11.9 & (8.5) & -32.2 & $(13.5)$ & -3.1 & $(17.2)$ & 4.6 & $(11.6)$ & -3.2 & $(13.8)$ & $-44.2^{*}$ & $(19.0)$ & -21.0 & $(16.2)$ \\
\hline & $\%$ correct & -0.6 & (1.9) & 2.3 & (2.1) & 0.8 & $(2.3)$ & $6.3^{*}$ & $(1.6)$ & -0.1 & $(2.1)$ & 3.0 & $(2.8)$ & 1.6 & $(2.4)$ & 0.2 & $(1.9)$ \\
\hline
\end{tabular}

${ }^{*} p<0.05,{ }^{\wedge} p<.1$. 
Table 2

Mean values (SEM) of dependent measures of Tower of London (ToL) after placebo and methylphenidate 10, 20 and $40 \mathrm{mg}$ ( $P L A, M 10, M 20$ and M40 respectively). (RT=response time)

\begin{tabular}{|c|c|c|c|c|c|c|c|c|c|}
\hline & & T160 & & & & & & & \\
\hline & & PLA & & M10 & & M20 & & M40 & \\
\hline \multirow{3}{*}{$\begin{array}{l}\text { Slope } \\
2 \text { steps }\end{array}$} & & 0.4 & $(0.0)$ & 0.4 & $(0.0)$ & 0.4 & $(0.3)$ & .4 & $(0.0)$ \\
\hline & $\mathrm{RT}$ & 4277 & (300) & 4356 & (366) & 4458 & (418) & 4760 & (508) \\
\hline & $\%$ correct & 95.8 & (2.3) & 95.9 & (2.1) & 95.1 & $(1.6)$ & 94.9 & (2.3) \\
\hline \multirow[t]{2}{*}{3 steps } & $\mathrm{RT}$ & 6039 & (502) & 6461 & (333) & 5835 & (446) & 6128 & (583) \\
\hline & $\%$ correct & 95.5 & (1.4) & 95.1 & (1.9) & $98.9^{*}$ & $(0.7)$ & 94.5 & (1.5) \\
\hline \multirow[t]{2}{*}{4 steps } & $\mathrm{RT}$ & 9416 & (895) & 9197 & (861) & 8636 & (715) & 9892 & (1153) \\
\hline & $\%$ correct & 91.6 & $(2.3)$ & 89.6 & $(2.1)$ & 93.5 & $(2.2)$ & 94.3 & $(2.7)$ \\
\hline \multirow[t]{2}{*}{5 steps } & $\mathrm{RT}$ & 13458 & (1007) & 15770 & (1231) & 15076 & (1321) & 15441 & (1291) \\
\hline & $\%$ correct & 84.1 & $(3.3)$ & $85.3^{*}$ & $(3.0)$ & 79.8 & $(2.7)$ & $85.4^{\wedge}$ & (3.3) \\
\hline
\end{tabular}

${ }^{*} p<0.05,{ }^{\wedge} p<.1$.

may lead to improved word learning test performance, the use of lists of 12 to 15 words in previous studies (Bray, et al., 2004; Hermens, et al., 2007; Kuypers \& Ramaekers, 2007) may have left no room for improvement.

There are ample indications of dopamine involvement in (spatial) working memory (Ellis, et al., 2007; Landau, Lal, O'Neil, Baker, \& Jagust, 2008; Robbins, 2005). Indeed, methylphenidate has been shown to improve spatial working memory performance (Elliott, et al., 1997; Mehta, et al., 2000). This effect was, however, not replicated in the present study. A possibly important difference between the self-ordered search tasks used in previous studies and the spatial working memory task used here is that encoding of spatial locations occurs under time pressure in the current task. Previous research has suggested that methylphenidate may induce activity and arousal in healthy volunteers (Clark, et al., 1986b; Elliott, et al., 1997). Hence, participants may have had difficulties to encode information due to over-arousal.

Methylphenidate generally improved performance of the set shifting task, as was indicated by both faster responses and a higher percentage of correct responses. Most effects were observed on the hear trials, suggesting that methylphenidate affected auditory attention more than visual attention. It is unclear why this should be the case. Our expectation that the effect of methylphenidate would be more pronounced in the reward parts of this task was not confirmed. Participants may not have paid attention to the ' $E$ ' sign, which indicated that they were in the reward condition. 
Methylphenidate improved stop signal task performance as was indicated mainly by faster responses. Effects were most prominent in the go trials. Faster responses (both in the set shifting and the stop signal task) may partly reflect the response readiness-enhancing effect of methylphenidate (Linssen, et al., 2012) increasing the speed of performance (Elliott, et al., 1997). Furthermore, taking into account the relatively high number of statistical comparisons being made in this study, some of the effects reported here including those on the Tower of London task may not be genuine drug effects. Therefore, the overall pattern of results was considered when describing drug effects. Thus, generally, MPH improved measures of declarative memory, attention and response inhibition.

The effects of methylphenidate were observed within a rather wide time window, between 90 and 270 min after drug intake. This likely reflects a relatively long lasting enhancement of dopamine levels in the blood after methylphenidate. Blood dopamine levels cannot be measured directly, but changes in prolactin level in blood are a good surrogate marker, since the prolactin level is known to rise as the dopamine level decreases and vice versa (Ben-Jonathan, 1985). Indeed, prolactin levels were decreased between 60 and $240 \mathrm{~min}$ after methylphenidate administration in this group of participants (Linssen, et al., 2011).

Most studies into effects of dopaminergic drugs on aspects of cognitive performance describe results according to an inverted $U$ curve when performance is mapped as a function of degree of neurotransmitter activity (Levy, 2009; Mehta, Goodyer, \& Sahakian, 2004). The design of the present study, using three doses of methylphenidate, the highest of which corresponds to $75 \%$ blocking of dopamine reuptake sites (Volkow, Wang, Fowler, Gatley, et al., 1998), should allow to reveal the inverted-U curve, if present. This would be reflected by improved performance at low and intermediate dosing levels and a drop in performance enhancement at the higher dose. However, the current findings suggest that methylphenidate influences memory consolidation in a monotonic positive fashion. Although dose-response relationships may differ between cognitive domains, it has been shown that methylphenidate effects on response readiness can also not be described in terms of the inverted $U$ function (Linssen, et al., 2011). Methylphenidate's enhancing effects on response readiness were observed even after a dose as low as $10 \mathrm{mg}$ and grew more consistent and prominent with increasing doses. Hence, our data challenge the existence of the inverted $U$ relationship, at least with respect to declarative memory consolidation and response readiness.

In conclusion, this was the first study to find dose-related effects of methylphenidate on declarative memory consolidation. This result is in line 
with earlier described findings of amphetamine-induced enhancement of memory consolidation. Improved performance was also observed on stop signal and set shifting tasks.

\section{Acknowledgements}

This study was carried out at Maastricht University, Maastricht, The Netherlands and was sponsored by F. Hoffmann-LaRoche Ltd., Basel, Switzerland. WJR is an employee of F. Hoffmann-La Roche Ltd. and was involved in designing the study, analysis and monitoring of the data and approval of the report.

The authors have full control of all primary data and agree to allow review of the data if requested.

The authors also would like to thank the following people for contributing to the study:

Dr. Cees van Leeuwen, Irene Lelieveld, Conny Quaedflieg, Saskia Schneider, Natalie Valle and Lizzy Vuurman.

The experiment complies with the current Dutch law. 
Chapter 3. Effects of methylphenidate and levodopa on (working) memory performance and Event Related Potentials in healthy volunteers

Submitted for publication

\begin{abstract}
Previous research has shown that methylphenidate (MPH) improves cognitive function in ADHD. The evidence for cognition enhancing effects of $M P H$ in healthy individuals is inconsistent. The current study aimed to investigate effects of MPH on declarative and working memory, specifically focusing on robustness and specificity of previous results. To study the dopaminergic contribution to $\mathrm{MPH}$ effects, effects of $40 \mathrm{mg}$ of $\mathrm{MPH}$ on memory performance were compared to $100 \mathrm{mg}$ of levodopa (LEV) in a placebo-controlled crossover study with 30 healthy volunteers. Memory performance testing included a word learning test and several working memory tests. Simultaneously with the word learning test, event-related brain potentials (ERPs) were measured. Subjective and physiological recodings were included as control measures. MPH decreased memory decay over a period of 24 hours. MPH and LEV had opposite effects on memory task performance and electrophysiological correlates. It is suggested that MPH enhances cognitive function under challenging conditions and that the LEV manipulation in this study did not lead to enhanced dopaminergic activity.
\end{abstract}




\section{Introduction}

Methylphenidate has been shown to not only reduce behavioral symptoms of children with $A D H D$, but also improve cognitive function in this group (Pietrzak, et al., 2006). Lately, interest in methylphenidate as a possible cognitive enhancer for the healthy population has increased (Repantis, et al., 2010; Sahakian \& Morein-Zamir, 2007; M. E. Smith \& Farah, 2011). Evidence for a global cognition enhancing effect of methylphenidate is lacking, as the results are reported to be mixed or inconsistent (Advokat, 2010; Repantis, et al., 2010; M. E. Smith \& Farah, 2011). However, enhanced performance following methylphenidate administration has been observed within specific domains of cognitive function such as speed of processing and working memory (Agay, et al., 2010; Elliott, et al., 1997; Halliday, et al., 1986; Kollins, et al., 1998; Linssen, et al., 2011; Mehta, et al., 2000). Recently, methylphenidate has been shown to have a dose-related performance enhancing effect on a word learning task in healthy volunteers (Linssen, et al., 2012). With this being the first study to find dose-related effects of methylphenidate on declarative memory consolidation, the robustness and specificity of these findings should be further studied.

Methylphenidate blocks the reuptake of the catecholamines dopamine and noradrenaline by binding to the transporters, thereby increasing catecholamine availability (Hannestad, et al., 2010; Kuczenski \& Segal, 1997; Volkow, Wang, Fowler, Gatley, et al., 1998). Whether the effects of methylphenidate on cognition are mediated by dopamine or noradrenaline is unclear. It is suggested that methylphenidate's potency may be higher for the noradrenaline- than the dopamine transporter (Kuczenski \& Segal, 1997). But as dopamine is also transported by the noradrenaline transporter, it is not clear if noradrenaline or dopamine is more affected by methylphenidate (Hannestad, et al., 2010; Pacholczyk, Blakely, \& Amara, 1991).

Previous research suggests that dopamine mediates working memory function. Working memory is impaired in conditions in which dopamine levels are suboptimal such as in Parkinson's disease (Dujardin \& Laurent, 2003; Nieoullon, 2002; Owen, Iddon, Hodges, Summers, \& Robbins, 1997) and can be restored by dopaminergic treatment (Costa, et al., 2003; Lewis, Slabosz, Robbins, Barker, \& Owen, 2005; Moustafa, Sherman, \& Frank, 2008). In healthy volunteers dopamine agonists enhance working memory performance whereas dopamine antagonists impair it (Elliott, et al., 1997; Luciana, et al., 1992; Mehta, et al., 1999). Declarative learning and memory are also modulated by dopamine. Associative learning and retention of these associations is enhanced by levodopa, the precursor of dopamine (Breitenstein, Floel, et al., 2006; Knecht, et al., 2004). Recognition of 
emotional material is impaired by sulpiride, a dopamine antagonist (Mehta, Hinton, Montgomery, Bantick, \& Grasby, 2005).

Noradrenaline is also thought to play a role in memory (Chamberlain, Muller, Blackwell, Robbins, \& Sahakian, 2006; Kobayashi \& Yasoshima, 2001). Noradrenaline antagonists, such as the beta-blocker propranolol impair working memory and emotional memory (Chamberlain, et al., 2006; U. Muller, et al., 2005; Oei, Tollenaar, Elzinga, \& Spinhoven, 2010; van Stegeren, et al., 2005). However the exact nature of the role of noradrenaline in memory is relatively poorly understood.

Hence, both modulation of noradrenaline- and dopamine alters cognitive function and methylphenidate's effect on memory consolidation may be mediated through either dopamine, noradrenaline or both. To study the contribution of each of these neurotransmitters to methylphenidate's effects on cognitive function, methylphenidate should be contrasted with pure dopaminergic and/or noradrenergic drugs. As discussed above, catecholamine-transporter blockers, even if specifically blocking only either transporter type, will affect both neurotransmitters. In the current study the cognitive effects of methylphenidate were compared to those of levodopa. Levodopa is used to treat Parkinson's disease (Nutt, 2008) and is a dopamine precursor which is converted to dopamine both in the peripheral as well as the central nervous system (Julien, 2005), increasing dopamine availability in body and brain. While the mechanism of increasing dopamine levels in the brain is different from that of methylphenidate, levodopa administration allows the study of purely dopaminergic effects on cognition without interfering with noradrenaline levels.

In Parkinson's disease, levodopa's effects on cognitive function have been reported to differ between patients (Kulisevsky, 2000). Reported effects of levodopa on cognition range from impairment and no effect to better performance (Ghilardi, et al., 2007; Shohamy, Myers, Geghman, Sage, \& Gluck, 2006; Shohamy, Myers, Grossman, Sage, \& Gluck, 2005). In healthy volunteers levodopa has been shown to improve motor memory formation (Floel, et al., 2005; Floel, et al., 2008). Furthermore accelerated associative learning and better retention of pseudowords were observed in healthy volunteers who received levodopa over five training days (Breitenstein, Floel, et al., 2006; Knecht, et al., 2004).

The current study aimed to replicate and extend the previous finding of enhanced declarative memory consolidation in healthy volunteers after methylphenidate. In order to study the dopaminergic contribution to the methylphenidate effect on the word learning test, the effect of a $40 \mathrm{mg}$ dose of methylphenidate was compared to that of $100 \mathrm{mg}$ of levodopa in a placebo controlled crossover designed study with 30 healthy volunteers. It was 
hypothesised that the effect of levodopa would be similar to that of methylphenidate, as levodopa has been shown to have the potential to improve retention of verbal information (Breitenstein, Floel, et al., 2006; Knecht, et al., 2004). Such a result would suggest a major role for dopamine in the memory enhancing effect of methylphenidate. Besides a standard word learning test, additional memory tasks were administered including spatial and verbal (working) memory tasks to investigate the specificity of the memory enhancing effect. Heart rate, blood pressure and subjective measures were included as control measures.

\section{Method}

\section{Participants}

Thirty healthy volunteers (20 male, 10 female, mean age $=20.7, S D=2.3$, range $=18-28$ ) were selected and paid to participate. Participants were recruited by means of local advertisements. Prescreening occurred using a medical history questionnaire and was followed by medical examination.

The main inclusion criteria were: between 18 and 45 years of age, body mass index between 18 and $30 \mathrm{~kg} / \mathrm{m}^{2}$ and right-handedness. The main exclusion criteria were history or presence of mental or physical disorders, consumption of more than 21 alcohol units per week or more than five caffeine-containing drinks per day, pregnancy or lactation, use of medication other than oral contraceptives, use of recreational drugs from 2 weeks before until the end of the experiment.

All subjects gave written informed consent. The study was carried out in accordance with the Declaration of Helsinki and approved by the medical ethical committee of Maastricht University.

\section{Design}

The study was conducted according to a double-blind, placebo-controlled, three-way crossover design. Between the testing days, a period of at least 48 hours elapsed, but generally, testing days were scheduled approximately one week apart. Each participant received one of three single treatments including placebo (PLA), $40 \mathrm{mg}$ of methylphenidate (MPH) and $125 \mathrm{mg}$ of levodopa/carbidopa (LEV) on each testing day. LEV was given in combination with $10 \mathrm{mg}$ of domperidone (a peripheral dopamine antagonist) to prevent nausea. Six different treatment sequences were used, with each sequence occurring 3 or 4 times. 


\section{Dependent measures}

Visual verbal learning test

This task is an adapted version of Rey's Auditory Verbal Learning Test (Lezak, 1995). The VVLT was used to measure declarative memory. It included 30 words that were presented on a computer screen (stimulus presentation time and interstimulus interval were $1.000 \mathrm{~ms}$, (Klaassen, et al., 2002)). This presentation was repeated three times using the same sequence of words, each time followed by immediate free recall of all remembered words. Thirty minutes after immediate free recall of the final series, participants were subjected to a delayed recall test and a recognition test. During the latter, 30 words were presented, 15 of which were previously presented and another 15 that were new. The delay was filled with other tasks. Twenty-four hours later participants were again subjected to a delayed recall and recognition test. Outcome measures were total number of correctly recalled words during immediate recall (summed over three trials) and number of correctly recalled words during delayed recall tests. The electrophysiological dependent measures were amplitude and latency of the P3a, P3b and P600 ERP components, measured during encoding (averaged across three trials). P3a and $\mathrm{P} 3 \mathrm{~b}$ are two subcomponents of the $\mathrm{P} 300$, which is interpreted to brain activity related to updating the mental representation of incoming stimuli (Polich, 2007; Polich \& Criado, 2006). P3a is induced by novel or unexpected stimuli, while $\mathrm{P} 3 \mathrm{~b}$ is associated with context updating and subsequent memory storage (Coull, 1998; Polich, 2007). P600, sometimes referred to as late positive component or positive slow wave has been associated with several higher-level functions, including syntactic processing, item recognition and working memory capacity (Chan, Chan, Kwan, Ting, \& Chui, 2012; Friedman \& Johnson, 2000; Lefebvre, Marchand, Eskes, \& Connolly, 2005; Mecklinger, 2010; Swaab, Ledoux, Camblin, \& Boudewyn, 2012).

The behavioral dependent measures of the recognition test were median reaction time, measured in milliseconds and sensitivity $\left(A^{\prime}\right) . A^{\prime}$ is calculated as follows: $A^{\prime}=1-1 / 4[\mathrm{fr} / \mathrm{cr}+(1-\mathrm{cr} / 1-\mathrm{fr})]$ with $\mathrm{fr}=$ falsely recognized words and $\mathrm{cr}=$ correctly recognized words. The electrophysiological dependent measures of the recognition task were amplitude and latency of the P3a, P3b and P600 ERP components.

\section{Sternberg memory scanning task}

Sternberg's memory scanning test assesses the speed of scanning items maintained in working memory (Sternberg, 1966). In the current test, somewhat modified compared to the original, participants were briefly shown a set of 1, 2 or 4 unrelated consonants and were told to memorize them. This set is labeled the memory set. Then a series of 48 letters was sequentially 
displayed on a computer screen (stimulus presentation time was $1000 \mathrm{~ms}$ and interstimulus interval was $2000 \mathrm{~ms}$ ). The participants were required to indicate if the presented items matched those of the memory set by pressing either of two buttons. Half of the presented stimuli were part of the memory set. In this task reaction time increases linearly with the workload; that is, the number of items in the memory set. The slope of this function is an indication of the speed of scanning of short-term memory, whereas intercept is a measure of psychomotor speed. The dependent variables were slope, intercept, reaction time (in $\mathrm{ms}$ ), and accuracy (indicated by a number between 0 and 1 with 0 indicating 100\% incorrect and 1 indicating 100\% correct).

\section{Paired associates learning}

The visual paired associates learning test requires participants to learn the association between an object and it's location. On the first trial, two boxes were displayed on the screen and opened one by one. Each box contained a different object. The objects were then displayed in the middle of the screen, one at a time, and the subject was asked to click the box that contained the object. On each consecutive trial the number of boxes increased by one if the subject made no error (locations and objects differed between the trials) and decreased by one if the subject made an error, until a total of three errors was made, at which point the task was ended. The outcome measures of this test were maximum number of paired associates that was correctly recalled and mean number of paired associates that was correctly recalled across trials.

\section{Spatial working memory task}

The 'Object relocation' program was used as a spatial working memory task (Kessels, et al., 1999; Kessels, et al., 2000). In this task, participants were required to relocate ten visual stimuli (small drawings of objects such as a car or a flower) that were presented on a computer screen in a $15-\mathrm{cm}^{2}$ sized square to their original location. The task consisted of two parts. In the first part, called 'object to position' or OTP, participants had 15 seconds time to memorize the locations of the objects and were asked to drag the objects to the correct positions, which were cued with black dots. In the second part, the combined (COM) part, participants had 30 seconds to memorize the locations and this time, there were no cues to indicate the previous positions. Participants were instructed to place the objects in their original position as accurately as possible. In both parts, there was no time limit for the relocation of the stimuli. The dependent variables were percentage correct for OTP and percentage correct, absolute error and positional fit for COM. In 
the COM part, percentage correct was calculated such that relocation would be correct when the object was placed within $20 \mathrm{~mm}$ from the original location. Absolute error was the absolute distance in millimeters an object was relocated away from its original position. Positional fit measured the ability to remember the locations per se, independent from the ability to remember which object was in each location and computed as the best fit score yielding the smallest distance error for the stimulus as a whole.

\section{Subjective measures}

Participants completed two rating scales of subjective effects, the Profile of Mood States (POMS; (MCNair, Lorr, \& Droppleman, 1992)) and the Bond and Lader (B\&L) visual analogue scales (Bond \& Lader, 1974). These rating scales consisted of 16 (B\&L) or 32 (POMS) bipolar items. Each item consisted of two words describing mood states. Participants had to score their preference for either of the items in accordance with their mood by marking the line closer to one of the two words. Dependent measures were factor scores on the dimensions Alertness (B\&L), Depression, Anger, Fatigue, Vigour and Tension (POMS). Both rating scales were administered at four time points (T0, T50, T130 and T180) on each testing day.

\section{Physiological measures}

Physiological measures included systolic and diastolic blood pressure and heart rate and were taken at four time points (T0, T50, T130 and T180) on each testing day.

\section{Electroencephalography}

During the visual verbal learning task, EEG was recorded using 11 electrodes attached to a cap according to the international 10-20 system (Jasper, 1958) at the Fz, F3, F4, Cz, C3, C4, C3', C4', Pz, P3 and P4 electrode positions. The ground electrode was placed on the $\mathrm{AFz}$ location, on the forehead. Two electrodes placed on the mastoids served as reference channels. An electrooculogram (EOG) was measured bipolarly vertically above and below the right eye and horizontally next to the right and the left eye. Data were sampled at $250 \mathrm{~Hz}$ and filtered online between 0.05 and $100 \mathrm{~Hz}$. Offline, data were screened for artefacts and filtered between 1 and $30 \mathrm{~Hz}$. ERPs were extracted by stimulus-locked averaging of epochs from $100 \mathrm{~ms}$ before to $1000 \mathrm{~ms}$ after stimulus presentation. In the ERPs extracted from the electroencephalogram during encoding several peaks could be discerned. P3a and P3b were respectively determined as the highest amplitude within 200-350 and 280-420 ms after stimulus onset, while checking that two distinct peaks were picked by the program. The P600 was determined as the highest amplitude between 
450 and 650 ms after stimulus onset. In addition, the latencies of these peaks were included in the analysis. In the ERPs measured during the recognition task, the same components were determined, with P3a and P3b between 200$300 \mathrm{~ms}$ and $380-420 \mathrm{~ms}$ after stimulus onset and P600 within 450-650 ms. The latencies of these peaks were included in the analysis.

\section{Procedure}

The current study was integrated in a larger study on the effects of methylphenidate and levodopa on cognition and psychomotor function. A training session in which the tasks were practiced took place within 2 weeks before the first testing day. Participants abstained from alcohol during the last $24 \mathrm{~h}$ prior to each testing day. On testing days, participants were either collected at their home or came by public transport. They arrived at the lab in fasted condition at either $8.30 \mathrm{am}$ or 9:00 am. Due to the differences in Tmax of the medication, drug administration occurred at different time points, $M P H$ at $\mathrm{T}=0$ (in minutes) and $\mathrm{LEV}$ at $\mathrm{T}=60$. Domperidon was administered at $T=30$. To ensure double blinding, participants received identically appearing capsules at all time points on each testing day. Participants were given a standardized meal for breakfast. Then, the electrode cap was placed and participants could relax until actual testing started at $T=120$. First, a baseline EEG measurement was taken, followed by cognitive testing in the following order: Visual verbal learning test, Sternberg memory scanning task, delayed recall and recognition test of the visual verbal learning test, paired associates learning and spatial working memory task. In addition, two blood samples were taken at $T=120$ and $T=180$. Subjective and physiological measures were taken at four time points (T0, T50, T130 and T180). At the end of the testing day, participants were returned to their home by car.

\section{Data analysis}

The part of the data-analysis aimed at testing whether previous results were replicated consisted of one-sided paired t-tests between MPH and PLA.

Next, the LEV data were entered in the analysis. Data were analyzed using a repeated measures analysis of variance (ANOVA), with the within subjects factor treatment ( 3 levels: PLA, MPH and LEV). For the EEG data additional within subjects factors were electrode ( 3 levels: $\mathrm{Fz}, \mathrm{Cz}, \mathrm{Pz}$ ) and trial ( 3 levels for the three presentations of the word list).

Since treatment effects of MPH and LEV were expected to go into the same direction, the main effects of treatment, electrode and trial were not evaluated. Instead, a priori planned comparisons between each drug relative to PLA were tested for significance at alpha $<.05$ using LSD correction. 
Because of the different nature of the subjective and physiological measures, effects on these measures were assessed by paired $t$-tests between either drug vs. PLA on difference scores (relative to morning baseline).

\section{Results}

\section{Visual verbal learning test}

Task performance

Mean values of dependent measures of the VVLT and p-values of the planned comparisons are presented in Table 1.

First, replication of previous results was tested by means of a one sided paired t-test between MPH and PLA. MPH did not affect immediate or 30 minute delayed recall. However, when participants learned the words when under the influence of $\mathrm{MPH}$, they remembered significantly more words at 24 hour delayed recall, compared to PLA $\left(\mathrm{t}_{29}=-2.033, \mathrm{p}<.05\right.$, one-sided). None of the variables of the recognition tests was affected by $\mathrm{MPH}$.

Next, effects of PLA, MPH and LEV were tested in a repeated measures ANOVA with planned comparisons between the two drug conditions and PLA (see Table 1). This analysis revealed a near significant difference between PLA and MPH $(p<.052)$ in line with the previously mentioned t-test showing better memory recall at the $24 \mathrm{~h}$ delay after methylphenidate. Although comparisons between LEV and PLA did not reveal significant differences on any of the variables, inspection of the averages suggested that LEV and MPH effects may not go in the same direction. Post hoc analysis, comparing LEV to MPH revealed that performance on the 24h delayed recall was significantly better after MPH compared to LEV $(p=.049)$. Again, the recognition test was not affected by either MPH or LEV.

\section{ERP measures}

Grand average ERPs, averaged over the three learning trials after PLA, MPH and LEV are shown in Figure 1. A repeated measures ANOVA with treatment, electrode and trial as within subjects factors was run on these data. This analysis showed that P3b latency was delayed after LEV relative to PLA (PLA: mean $(\mathrm{SEM})=340.8 \mathrm{~ms}$ (3.4); LEV: mean $(\mathrm{SEM})=351.3 \mathrm{~ms}$ (3.5); PLA vs. LEV: $\mathrm{p}<.002$ ). P600 amplitude was larger after LEV compared to PLA (PLA: mean $(\mathrm{SEM})=4.47 \mu \mathrm{V}(.37)$; LEV: mean $(\mathrm{SEM})=5.09 \mu \mathrm{V}(.31)$; PLA vs. LEV: $\mathrm{p}<.018)$.

Figure 2 depicts grand average ERPs measured during recognition testing after PLA, MPH and LEV. The analysis did not reveal significant differences between any of the treatments. 
Mean scores (SEM) on the Visual Verbal Learning test

\begin{tabular}{|c|c|c|c|c|c|c|c|c|c|}
\hline & \multicolumn{6}{|c|}{ Mean (SEM) scores on the VVLT } & \multicolumn{3}{|l|}{ Contrasts } \\
\hline & PLA & & $\mathrm{MPH}$ & & LEV & & PLA vs. MPH & PLA vs. LEV & MPH vs. LEV \\
\hline Immediate recall & 43.7 & $(2.0)$ & 43.0 & $(2.0)$ & 42.6 & $(2.3)$ & .726 & .567 & .823 \\
\hline Delayed recall $30 \mathrm{~min}$ & 17.0 & $(1.1)$ & 17.2 & $(1.1)$ & 16.6 & $(1.1)$ & .855 & .566 & .504 \\
\hline Delayed recall $24 \mathrm{~h}$ & 13.0 & $(1.2)$ & 14.7 & $(1.2)$ & 13.1 & $(1.1)$ & .051 & .927 & $.049^{*}$ \\
\hline \multicolumn{10}{|l|}{ Recognition } \\
\hline Reaction time (ms) $30 \mathrm{~min}$ & 660 & $(1.5)$ & 664 & $(16)$ & 677 & $(18)$ & .803 & .320 & .413 \\
\hline Sensitivity $\left(A^{\prime}\right) 30 \mathrm{~min}$ & 2.7 & $(0.1)$ & 2.7 & $(0.1)$ & 2.6 & $(0.1)$ & .863 & .305 & .431 \\
\hline Reaction time $(\mathrm{ms}) 24 \mathrm{~h}$ & 693 & $(23)$ & 669 & (19) & 685 & $(22)$ & .194 & .736 & .447 \\
\hline Sensitivity $\left(\mathrm{A}^{\prime}\right) 24 \mathrm{~h}$ & 2.5 & $(0.1)$ & 2.5 & $(0.1)$ & 2.4 & $(0.1)$ & .777 & .064 & .095 \\
\hline
\end{tabular}

${ }^{*} p<.05$ 


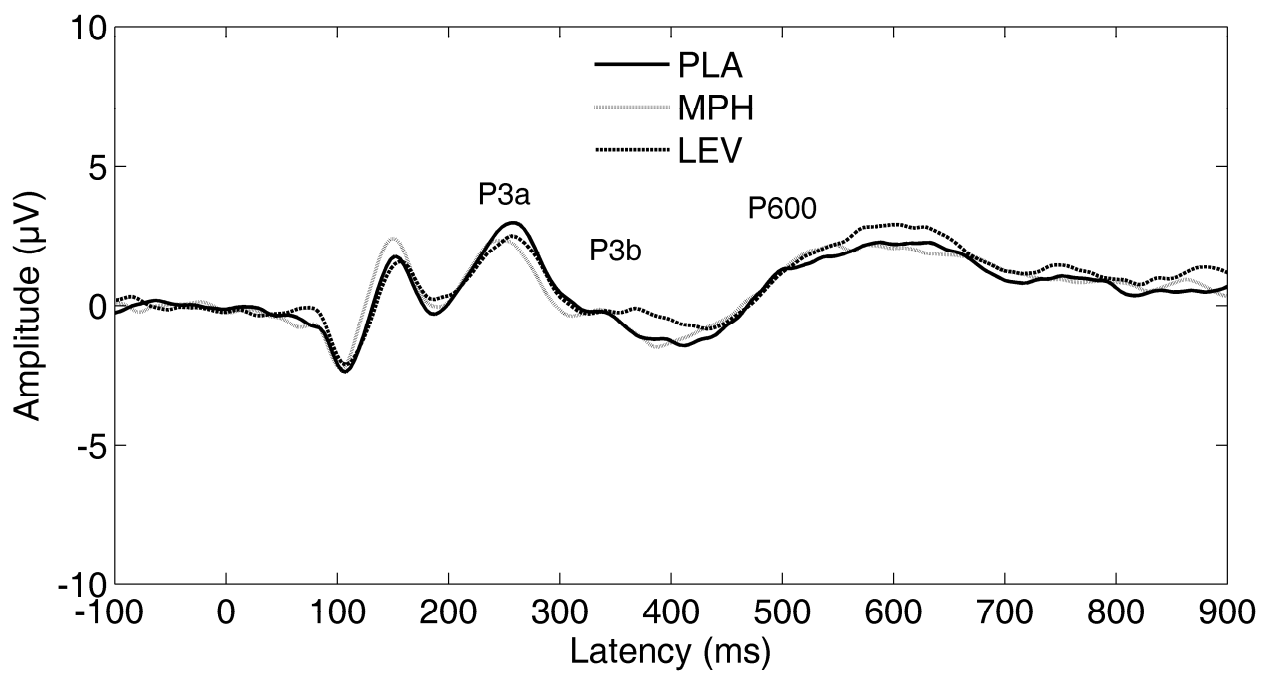

Figurure 1. Grand average ERPs showing the P3a, P3b and P600 during the encoding phase (averaged across three learning trials) of the visual verbal learning test. The ERPs depicted were recorded at $\mathrm{Cz}$.

Behavioral data revealed improved $24 \mathrm{~h}$ delayed recall performance after MPH relative to PLA and LEV, but this result was not reflected by the ERP results. It was therefore decided to do a further analysis only including the EEG of words that were recalled at the $24 \mathrm{~h}$ delayed recall to see if the differences in performance would be reflected by the ERPs at encoding (Grand averages are shown in Figure 3). This analysis again revealed delayed P3b latency after LEV compared to PLA (PLA: mean (SEM)=343.0 ms (5.4); LEV: mean (SEM) $=356.1$ ms (4.7); PLA vs. LEV: $p<.049)$. P600 amplitude was larger after LEV relative to $\mathrm{MPH}$ (MPH: mean (SEM) $=5.45 \mu \mathrm{V}(.51)$; LEV: mean (SEM) $=7.44 \mu \mathrm{V}(.54) ; \mathrm{MPH}$ vs. LEV: $\mathrm{p}<.006)$. P600 latency was delayed after LEV relative to MPH (MPH: mean $(\mathrm{SEM})=558.9 \mathrm{~ms}(8.5)$; LEV: mean $(\mathrm{SEM})=577.0 \mathrm{~ms}$ (6.3); MPH vs. LEV: $\mathrm{p}<.037)$.

\section{Other cognitive tasks}

Mean values of dependent measures of the Sternberg memory scanning task, Paired Associates learning and the spatial working memory task are presented in Table 2.

MPH and LEV influences on the Sternberg Memory Scanning task variables did not differ significantly from PLA. However, inspection of the averages again suggested a difference between the two drug conditions. The contrast MPH vs. LEV revealed that responses were significantly faster after $M P H$ relative to LEV (see Table 2 ). 
On the Paired Associates Learning task there was again no difference between either drug condition and PLA, while the two drug conditions did differ significantly from each other. Both max and mean values were higher for MPH compared to LEV.

- Performance on the spatial working memory task was similar in all conditions.

a

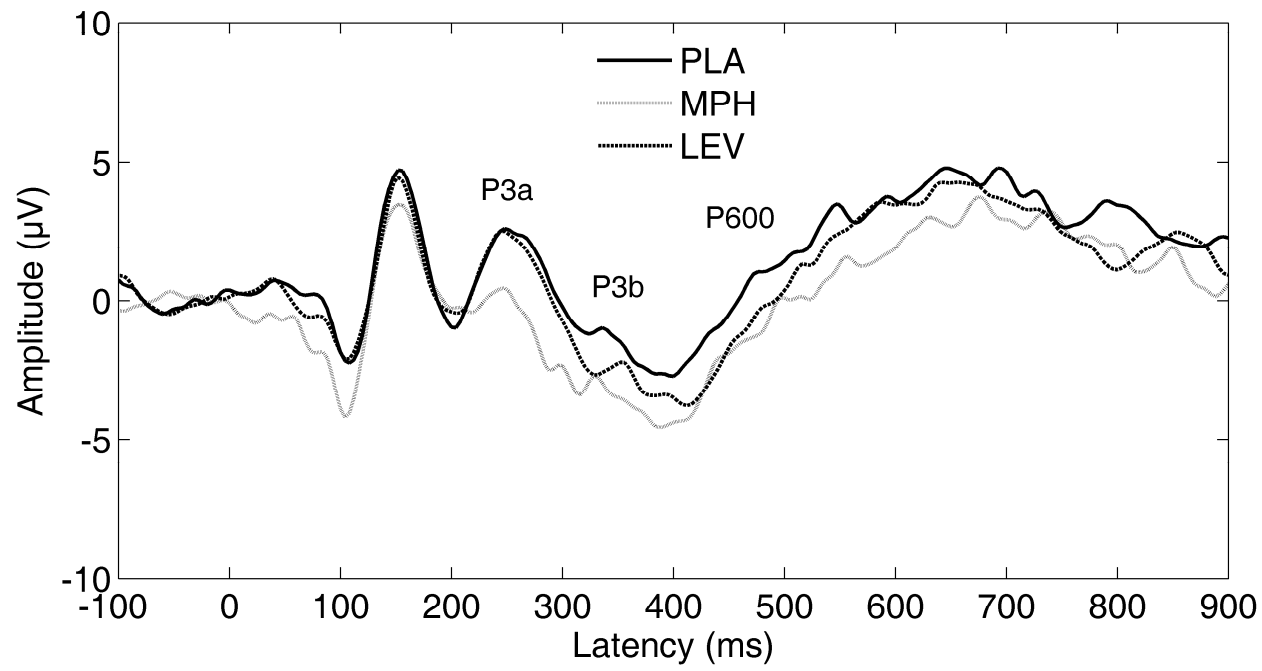

b

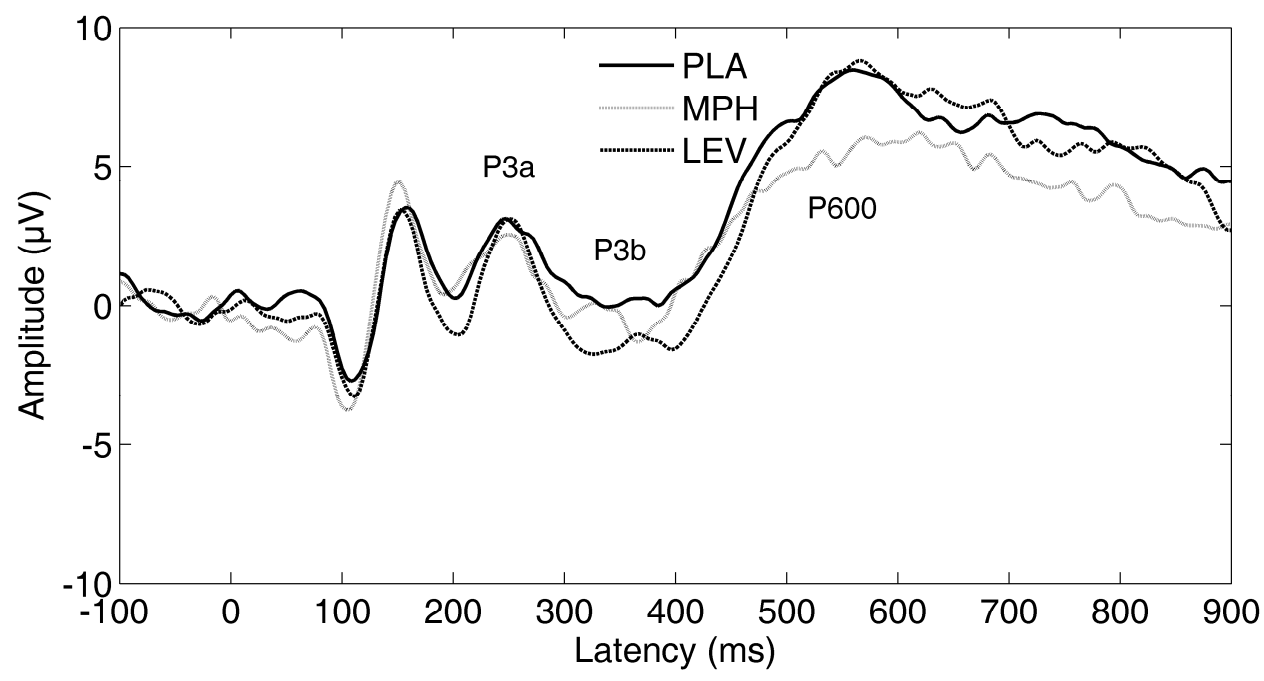

Figure 2. Grand average ERPs showing the P3a, P3b and P600 during the recognition phase of the visual verbal learning test for old (a) and new (b) items. The ERPs depicted were recorded at Cz. 


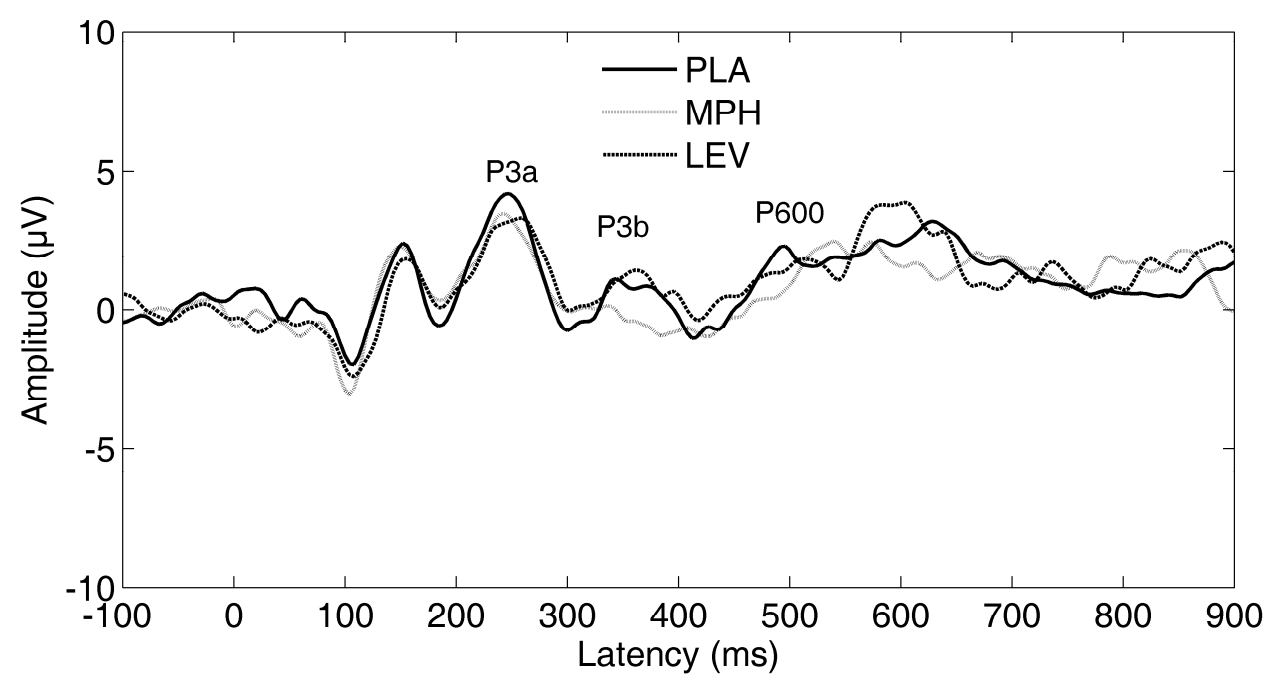

Figure 3. Grand average ERPs showing the P3a, P3b and P600 during encoding of words that were remembered at $24 \mathrm{~h}$ delayed recall. of the visual verbal learning test. The ERPs depicted were recorded at $\mathrm{Cz}$.

\section{Subjective measures}

Analysis of POMS factor scores indicated that after methylphenidate, participants experienced less fatigue at 1 and 3 hours after drug intake $(p<.015$ and $p<.020$ respectively). Methylphenidate also caused an increase in reported tension at 1 hour and 2 hours after administration $(\mathrm{p}<.041$ and $\mathrm{p}<.016$ respectively). Factor scores indicated less vigour at 1 and 3 hours after methylphenidate intake $(p<.004$ and $p<.006$ respectively). The Bond \& Lader factor scores indicated that participants felt more alert 3 hours after MPH intake $(p<.038)$. LEV did not affect subjective feelings as reported by participants.

\section{Physiological measures}

MPH significantly increased heart rate compared to PLA at all time points (T50: $\mathrm{p}<.001 ; \mathrm{T} 130: \mathrm{p}<.001 ; \mathrm{T} 180: \mathrm{p}<.001)$. Furthermore, compared to PLA, MPH also significantly increased systolic at all time points (T50: $p<.001 ; T 130$ : $\mathrm{p}<.001 ; \mathrm{T} 180: \mathrm{p}<.001)$ and diastolic blood pressure (T50: $\mathrm{p}<.001 ; \mathrm{T} 130$ : $\mathrm{p}<.003 ; \mathrm{T} 180: \mathrm{p}<.003)$. LEV did not affect heart rate and systolic blood pressure at $\mathrm{T} 130$, but significantly decreased diastolic blood pressure relative to PLA $(p<.048)$. In both PLA and LEV, heart rate decreased from T0 to T180, but this decrease was significantly smaller after LEV $(p<.036)$. At T180, there were no effects of LEV on blood pressure. Please note that changes in 
Mean scores (SEM) on the Sternberg Memory Scanning, Paired Associates Learning and Spatial working memory tests

\begin{tabular}{|c|c|c|c|c|c|c|c|c|c|}
\hline & \multicolumn{6}{|c|}{ Mean (SEM) scores } & \multicolumn{3}{|l|}{ Contrasts } \\
\hline & PLA & & $\mathrm{MPH}$ & & LEV & & PLA vs. MPH & PLA vs. LEV & MPH VS. LEV \\
\hline \multicolumn{10}{|c|}{ Sternberg Memory Scanning } \\
\hline slope & 40 & (3) & 37 & (3) & 40 & (3) & .163 & .923 & .224 \\
\hline intercept & 359 & (8) & 352 & $(10)$ & 361 & $(8)$ & .450 & .758 & .296 \\
\hline accuracy & 0.97 & $(0.00)$ & 0.97 & $(0.00)$ & 0.97 & $(0.00)$ & .252 & .488 & .191 \\
\hline reaction time (ms) & 471 & $(11)$ & 456 & $(11)$ & 476 & $(10)$ & .097 & .380 & $.029^{*}$ \\
\hline \multicolumn{10}{|c|}{ Paired Associates Learning } \\
\hline maximum & 5.7 & $(0.1)$ & 6.0 & $(0.2)$ & 5.6 & $(0.2)$ & .222 & .344 & $.037^{*}$ \\
\hline mean & 4.0 & $(0.1)$ & 4.2 & $(0.1)$ & 3.9 & $(0.1)$ & .165 & .379 & $.024^{*}$ \\
\hline \multicolumn{10}{|c|}{ Spatial working memory } \\
\hline OTP \% correct & 80.5 & $(2.5)$ & 83.5 & $(2.6)$ & 80.8 & $(2.7)$ & .359 & .912 & .462 \\
\hline COM \% correct & 80.7 & $(2.2)$ & 78.5 & $(2.6)$ & 80.8 & $(2.4)$ & .473 & .949 & .469 \\
\hline COM abs err & 154.9 & $(8.4)$ & 166.7 & $(13.1)$ & 156.4 & $(10.6)$ & .372 & .865 & .487 \\
\hline COM pos fit & 124.6 & $(4.9)$ & 125.0 & $(5.2)$ & 117.2 & $(4.3)$ & .946 & .117 & .156 \\
\hline
\end{tabular}


physiological measure reported here are relatively small and all values are still within a normal clinical range for healthy individuals.

\section{Discussion}

This study aimed to replicate and extend the previous finding of MPH induced enhancement of declarative memory consolidation, by testing memory performance of healthy volunteers under influence of PLA, MPH or LEV. MPH failed to improve 30 minute delayed recall of word lists. However, performance on the 24 hour delayed recall was better when words had been learned under the influence of MPH compared to PLA and LEV. The P3b and P600 latencies were delayed and P600 amplitude was larger after LEV compared to PLA and MPH. MPH speeded response times on the Sternberg Memory Scanning task and improved performance on the Paired Associates Learning task, relative to LEV. The Spatial working memory task was not affected by the treatments.

A recent study showed that MPH improved 30 minute delayed word recall. That finding was not replicated by the present study. The discrepancy between the current and previous findings may be explained by differences in the study procedures. Although timing of task performance relative to dosing was the same in both studies, the hours before administration of the word learning task were spent rather differently. While participants in the previous study had already been subjected to 3.5 hours of cognitive testing, the participants in the present study were well rested before word learning task performance. As MPH is known to reverse the performance disruptive effects of sleep deprivation (Bishop, et al., 1997), MPH may have similarly reduced the impact of fatigue, induced by 3.5 hours of computerized (cognitive) testing, on cognitive performance in the previous study.

The finding that when participants learned the word lists under the influence of MPH remembered more words during the second delayed recall after 24 hours, compared to the PLA condition, suggests that MPH led to less memory decay between 30 minutes and 24 hours after word learning. To remember the word lists over a 24 hour period could be considered rather challenging. Combined with the observations on the first delayed recall test in the present and previous study discussed above, this finding may suggest that $\mathrm{MPH}$ has potential to improve cognitive performance in challenging conditions. If this is true, it has implications for the use of MPH by healthy individuals who wish to enhance their cognitive performance. This may be effective, but only when one needs to perform under demanding circumstances, including fatigue or a particularly difficult task. 
It was hypothesized that LEV and MPH would both enhance memory consolidation compared to PLA, which would suggest that MPH effects would be primarily mediated by dopamine activity. However, like MPH, LEV failed to improve 30 minute delayed recall. In addition, LEV also failed to enhance 24 hour delayed recall. In fact the averages even suggest that MPH and LEV have opposite effects on memory, although statistical testing only confirmed that MPH improved performance at $24 \mathrm{~h}$ delayed recall relative to PLA and LEV.

Latencies of two ERP components, P3b and P600 were delayed after LEV relative to PLA and MPH. As P300 latency is associated with mental speed (Polich, 2012), and P300 and P600 are closely related (Swaab, et al., 2012), these effects suggest that LEV slows down memory processing. P600 amplitude was larger after LEV compared to PLA and MPH. Although P600 is usually related to syntactic processing (Swaab, et al., 2012), its similarity to the P300 with respect to sensitivity to stimulus salience, probability and task relevance suggest that an increase in amplitude may reflect increased effort to memorize items.

Based on previous findings of dopamine involvement in working memory (Ellis, et al., 2007; Landau, et al., 2008; Robbins, 2005) and better working memory performance after MPH (Elliott, et al., 1997; Mehta, et al., 2000), MPH and LEV were both hypothesized to improve performance on the Sternberg memory scanning, Paired associates learning and spatial working memory tasks relative to PLA. The present findings were not in agreement with these hypotheses as performance in the MPH condition nor the LEV condition differed from performance under PLA. Contrary to our expectations, $\mathrm{MPH}$ and LEV conditions differed from each other on several working memory related variables, suggesting that $\mathrm{MPH}$ slightly improved working memory while LEV may have minor deleterious effects on working memory performance.

The results from the current study thus suggest that MPH has minor though positive effects on cognitive function, which is in line with our expectations. The LEV effects, on the other hand, were not. In contrast with previous findings, LEV did not enhance performance. Rather, relative to MPH, LEV appeared to have disadvantageous effects on cognitive performance, partly reflected by general slowing after LEV. Hence, these findings do not support the hypothesis that dopamine plays a major role in cognition enhancing effects of MPH. However, based on this study, the role of dopamine in MPH effects can neither be declined. There are several other explanations for these results that need to be taken into account, and will be discussed below.

LEV has the potential to enhance presynaptic dopamine availability and thus may affect both tonic and phasic dopaminergic processes. This is a 
crucial difference between LEV vs. MPH and other dopamine agonists, which only affect tonic dopaminergic neurotransmission (Breitenstein, Korsukewitz, et al., 2006; Seeman \& Madras, 2002). Tonic and phasic activity differentially affect extracellular dopamine availability. Increased tonic dopamine activity on postsynaptic receptors and autoreceptors reduces phasic dopamine signals (Breitenstein, Korsukewitz, et al., 2006; Seeman \& Madras, 2002). Dopaminergic agents have biphasic effects, where low doses reduce phasic dopamine release while high doses induce widespread stimulation of postsynaptic receptors, prevailing over presynaptic inhibition (Seeman \& Madras, 2002). The LEV dose may thus have been too low, and have failed to enhance dopamine availability. The observed lack of subjective effects of LEV suggests there was a net null effect of LEV on dopaminergic activity. Unfortunately there are no prolactin data (prolactin levels decrease under the influence of increased dopamine availability) to support this suggestion as the concomitant treatment with domperidon, a peripheral dopamine antagonist, would compromise the blood test results.

If, in contrast with the previous suggestion, it would be assumed that LEV did enhance dopamine availability, another explanation of the current results could be that the crucial factor determining the cognition enhancing effects of MPH is the inhibition of both dopamine and noradrenaline reuptake. Due to the lack of availability of a pure noradrenergic drug, the MPH effects can only be compared to those of the purely dopaminergic treatment with LEV. The current design therefore leaves open two options: either the noradrenergic effects of MPH may be essential in performance enhancement or the combination of dopaminergic and noradrenergic effects are the key to enhanced performance on cognitive tasks. However, either explanation cannot account for the apparent detrimental effects of LEV relative to MPH. As discussed above, low doses of dopaminergic drugs may reduce phasic activitiy by stimulation of autoreceptors. It is therefore more likely that LEV dose was indeed too low and not only failed to enhanced dopamine activity but perhaps even decreased dopamine availability relative to $\mathrm{MPH}$, paralleling electrophysiological and behavioral results showing differential effects of MPH vs. LEV.

In sum, the current study showed that MPH led to decreased memory decay over a period of $24 \mathrm{~h}$ hours. Furthermore, MPH and LEV had opposite effects on memory task performance and electrophysiological correlates. It is suggested that MPH enhances cognitive function under challenging conditions and that the LEV manipulation in this study did not lead to enhanced dopaminergic activity. 
PART 1B: The effect Of Reduced dopamine LeVels ON COGNIT ION 

Chapter 4. Effects of tyrosine/phenylalanine depletion on

electrophysiological correlates of memory in healthy volunteers

Linssen, A. M., Riedel, W. J., \& Sambeth, A. (2011)

J Psychopharmacol, 25(2), 230-238

\section{Abstract}

Dopamine is well known for involvement in reinforcement, motor control and frontal lobe functions, such as attention and memory. Tyrosine/phenylalanine depletion (TPD) lowers dopamine synthesis and can therefore be used as a model to study the effects of low dopamine levels. This is the first study to assess the effect of TPD on memory performance and its electrophysiological correlates. In a double blind placebo (PLA)-controlled crossover design, 17 healthy volunteers (six males, 11 females) aged between 18 and 25 were tested after TPD and PLA. Working memory was assessed using a Sternberg memory scanning task (SMS) and episodic memory using the Visual Verbal Learning Test (VVLT). Simultaneously, event-related potentials (ERPs) were measured. The tyrosine and phenylalanine ratio was significantly reduced after TPD and increased after PLA. Working memory performance was not affected by TPD. However, ERP measures were affected by the treatment, indicating that TPD impaired stimulus processing during working memory performance. Episodic memory was not impaired after TPD. Again, alterations in ERP measures suggested adverse effects of TPD on memory-related processing. These results suggest that dopamine is involved in both working memory and episodic memory-related processing, although the effects are too small to be detected by performance measures. 


\section{Introduction}

Dopamine is one of the most thoroughly studied neurotransmitters. It is known to be involved in reinforcement and motor control (Nieoullon, 2002). In addition, evidence is accumulating that dopamine is likewise involved in cognitive function, including frontal lobe functions such as attention, planning and working memory (Cools \& Robbins, 2004). Previous research has shown that in diseases in which dopamine transmission is altered, such as Parkinson's Disease, working memory is disturbed (Nieoullon, 2002; Owen, et al., 1997). Dopaminergic treatment alleviates this problem (Costa, et al., 2003; Lewis, et al., 2005; Moustafa, et al., 2008). Similarly, dopamine elevating compounds improve working memory performance in healthy volunteers (Elliott, et al., 1997; Luciana \& Collins, 1997; Luciana, et al., 1992). Administration of dopamine lowering compounds, on the other hand, results in impairment of working memory (Luciana \& Collins, 1997; Mehta, et al., 1999).

A way to study dopamine function is to acutely lower dopamine levels in the brain by depleting its precursors, tyrosine and phenylalanine (Barrett $\&$ Leyton, 2004). Because these amino acids are transported into the brain by neutral amino acid transporters, amino acids have to compete with each other. Lowering the relative amount of one or two specific amino acids worsens their position in this competition, which thereby leads to decreased availability of these precursors in the brain (Barrett \& Leyton, 2004; McTavish, et al., 2001). Indeed, there is evidence that TPD reduces dopamine release, dopamine synthesis and attenuates induced elevation of dopamine levels (Bongiovanni, Newbould, \& Jaskiw, 2008; M. Le Masurier, Houston, Cowen, Grasby, \& Hume, 2004; Leyton, et al., 2004; McTavish, Cowen, \& Sharp, 1999; Montgomery, McTavish, Cowen, \& Grasby, 2003). The effect of TPD is anatomically selective and preferentially affects dopamine rich areas such as caudate and putamen while not affecting other regions such as frontal and cingulate cortex (M. Le Masurier, Cowen, \& Sharp, 2004).

The findings with respect to various cognitive functions have been very inconsistent; some studies found effects of TPD lowering scores on spatial recognition and spatial working memory tasks (Gijsman, et al., 2002; Harmer, McTavish, Clark, Goodwin, \& Cowen, 2001; Harrison, et al., 2004; Nathan, et al., 2002), whereas others did not (Ellis, et al., 2007; Lythe, Anderson, Deakin, Elliott, \& Strickland, 2005; Mehta, Gumaste, Montgomery, McTavish, \& Grasby, 2005), even though lower spatial working memory and planning accuracy scores were associated with decreased striatal dopamine levels (Mehta, Hinton, et al., 2005). Furthermore, TPD impairs vigilance and sustained attention when combined with serotonin depletion (Matrenza, et al., 2004), but TPD alone does not (Harrison, et al., 2004). Grevet et al. 
(2002) found impaired performance on delayed recall in Rey's auditory verbal learning test (RAVLT) after TPD (Grevet, et al., 2002), while Harrison et al. (2004) found no effect on a visual word learning task.

More recently, TPD has been used to study the relationship between dopamine function and mood. It has been suggested that TPD induces decreases in mood in healthy volunteers (Leyton, et al., 2000; McLean, Rubinsztein, Robbins, \& Sahakian, 2004; Ruhe, Mason, \& Schene, 2007). Furthermore, after TPD both healthy volunteers and recovered depressed patients display higher sensitivity to negative words and lower sensitivity to positive words in an affective Go/No-Go task (McLean, et al., 2004; Roiser, et al., 2005; Vrshek-Schallhorn, Wahlstrom, Benolkin, White, \& Luciana, 2006).

Research on the relationship between dopamine and ERP components is scarce. The available evidence, chiefly regarding P300, a component that is related to novelty processing and stimulus evaluation, is inconsistent (Coull, 1998; Farber, Beteleva, \& Ignat'eva, 2004; Prasher \& Findley, 1991; Stanzione, et al., 1991). For instance, $\mathrm{P} 300$ latency has been reported to be increased both in cases of enhanced as well as reduced dopamine transmission; delayed latency has been observed in PD and after administration of both dopamine antagonists as well as dopamine agonists (Prasher \& Findley, 1991; Stanzione, et al., 1991).

Because previous studies produced conflicting results with respect to the memory modulating effects of altered dopamine transmission, this study aimed to further explore the effects of TPD on memory function, including working memory and episodic memory. This was done by measuring performance using the Sternberg memory scanning task and a verbal learning task, and event-related potentials (ERPs) extracted from electroencephalography (EEG) recordings. As to our knowledge, no study currently exists that has combined TPD and EEG during cognitive processing. Because working memory deficits have been observed after depletion of dopamine in PD, the intake of dopamine antagonists and TPD (Gijsman, et al., 2002; Harmer, et al., 2001; Harrison, et al., 2004; Mehta, et al., 1999; Nathan, et al., 2002; Nieoullon, 2002), it was expected that depletion of tyrosine and phenylalanine would interfere with working memory function. Furthermore, it was hypothesized that TPD may disturb verbal learning (Grevet, et al., 2002) and possibly enhance sensitivity to negative words.

\section{Method}

\section{Participants}

Twenty-two healthy volunteers aged between 18 and 25 years were recruited by means of advertisement posters at Maastricht University. Preselection 
occurred on basis of pre-screening by telephone. Participants without any presence of psychiatric and neurologic diseases were subjected to a medical screening by means of a medical questionnaire. Inclusion criteria were normal binocular activity (corrected or uncorrected), a body mass index between 18.5 and 30 and signed informed consent.

Exclusion criteria were a history or presence of mental or physical disorders such as cardiac, hepatic, renal, pulmonary, neurological gastrointestinal, haematological or psychiatric illnesses, and sensory or motor deficits that could affect test performance. Furthermore, participants were excluded if pregnant or lactating, in case of drug abuse, when drinking more than 20 alcoholic beverages a week or more than five caffeine containing beverages a day.

Five participants did not complete the study because they had to vomit after intake of the amino-acid drink. The 17 participants ( 11 females, 6 males) who completed this study had a mean age of 21.1 years ( $S D=2.0$ years).

The study was approved by the ethical committee of Maastricht University and conducted in accordance with the ethical principles that have their origin in the Declaration of Helsinki 1975, revised Hong Kong 1989.

\section{Design}

The study was conducted according to a double-blind, placebo-controlled, three-way crossover design. The three testing sessions were spaced apart by a period of at least one week. On one occasion participants received a balanced amino acid drink (PLA). On the other two occasions they received an amino acid drink either lacking tyrosine and phenylalanine (TPD) or histidine (HID, as part of a larger research study (van Ruitenbeek, Sambeth, Vermeeren, Young, \& Riedel, 2009).

\section{Procedure}

The study was integrated in a larger study on the influence of TPD and HID on brain and behaviour. This section describes the procedure of the complete study.

Participants were not allowed to use drugs or medication, except oral contraceptives within two weeks before the first test-day until the end of the study. The participants were instructed to arrive at the lab in fasted condition. Furthermore they were requested to abstain from alcohol and caffeine from 9 p.m. the night before. Testing days started at either $8.30 \mathrm{am}$ or $10.30 \mathrm{am}$. A catheter was placed in order to collect blood samples, which were used to verify if depletion was successful. Blood samples were obtained at $0,2,4,6$ and 7 hours. After the first blood sample was collected, participants completed two questionnaires: Bond \& Lader and the complaint 
list. Subsequently participants received the amino acid drink, which had to be consumed within 15 minutes. This was followed by a break in which participants could consume water or caffeine free tea. After three hours participants had lunch. The lunch consisted of three slices of gluten free bread with honey, apple treacle or jam, an apple and a caffeine free soda drink.

Four and half hours after consumption of the drink, the EEG cap was placed. EEG recording started five hours after consumption of the drink with four minutes baseline measurement, two minutes with eyes open and two minutes with eyes closed. Thereafter participants were subjected to a test battery of approximately 90 minutes (with a 10 minute break in between), consisting of the following tests: Visual Verbal Learning Test, Critical Tracking Task, Sternberg Memory Scanning task, Visual Verbal Learning Test: delayed recall and delayed recognition, Choice Reaction Time task, Simple Reaction Time task, and an Oddball paradigm. In this study only the Sternberg Memory Scanning task and the Visual Verbal Learning Test are of interest. After completion of the test battery baseline EEG measurement was repeated. After the last blood sample was taken, the catheter was removed and participants completed the mood questionnaire and the complaint list a second time.

\section{Material}

\section{Drink composition}

The quantities of amino acids in the mixture, as given to male participants, were based on those used by Young et al. (Young, Smith, Pihl, \& Ervin, 1985). Female participants received $85 \%$ of this amount based on their average lower body weight (Ellenbogen, Young, Dean, Palmour, \& Benkelfat, 1996). The content of the TPD mixture was the same as the balanced drink except that it lacked tyrosine and phenylalanine. The amino acids were solved in $200 \mathrm{ml}$ (for males) or $170 \mathrm{ml}$ (for females) water.

\section{Blood samples}

Blood samples were collected via a catheter placed in the participants' dominant arm. After collection of the blood, the catheter was cleaned with heparin and blood samples were centrifuged for 5 minutes at $4{ }^{\circ} \mathrm{C}$ (at 4000 $\mathrm{rpm})$. Subsequently, $1 \mathrm{ml}$ of plasma was stored at $-20^{\circ} \mathrm{C}$ until performance of amino acid analysis (van Eijk, Rooyakkers, \& Deutz, 1993). Dependent measures were the percentage of change from T0 to T7 in both tyrosine and phenylalanine plasma levels and tyrosine and phenylalanine/ $\Sigma$ LNAA ratio. 


\section{Questionnaires}

Two questionnaires were used to asses mood; the Bond \& Lader Visual Analogue Scale (VAS) and the Complaint List. The VAS required participants to score bipolar items by marking a $100 \mathrm{~mm}$ line, with a normal state being indicated by marking the middle of the line. The dependent measures were total scores, measured in millimetres, on three dimensions: alertness, contentment and calmness. On the complaint list participants have to indicate, on a scale from 0 to 3 , to what extent they experienced the presented complaints, including for example nausea, headache and feeling cold. Dependent measure was the total score summed over the 31 items.

Tasks

Sternberg Memory Scanning task (SMS)

The memory scanning task (Sternberg, 1966) was used as a measure of working memory. In this task participants were required to memorize 1, 2 or 4 consonants. After memorization, 48 consonants were presented and participants were required to indicate if the presented items appeared in the memorized sequence. The stimuli were presented in six blocks of 48 items each. In the first three blocks workload increased, so participants had to memorize 1 letter in the first block, 2 letters in the second and 4 letters in the third block. In the fourth, fifth and sixth block this order was reversed. In this task reaction time increases linearly with the workload, that is, the number of items that has to be held in memory. The slope of this function is an indication of speed of scanning of short term memory, whereas the intercept is a measure of psychomotor speed. The behavioural dependent measures were slope, intercept, reaction time, measured in milliseconds, and accuracy indicated by a number between 0 and 1 , with 0 indicating that the answer was false and 1 indicating that the answer was right. The electrophysiological dependent measures were the amplitude and latency of the P150, N200, P3a and P3b ERP components.

\section{Visual verbal learning test (VVLT)}

This task is an adapted version of Rey's Auditory Verbal Learning Test (Lezak, 1995). The VVLT was used to measure episodic memory. It included 30 words, 12 of which are positive, 12 are negative and 6 are neutral, as was determined by valence ratings of word lists by a pool of healthy volunteers (Klaassen, et al., 2002). These words were presented on a computer screen three times in a row, each time followed by immediate free recall of all remembered words. After 30 minutes, participants were subjected to a delayed recall test and a recognition test. During the latter, 30 words were presented, 15 of which were previously presented and another 15 that were 
new. Outcome measures of the immediate and delayed recall tests were hits (correctly recalled words), false alarms (words named that were not on the (ist) and doubles (words named twice). In addition, performance was assessed taking into account the valence of the words, by taking the same measures for each of the valence categories separately.

The behavioural dependent measures of the recognition test were reaction time, measured in milliseconds, accuracy, indicated by a number between 0 and 1 , with 0 indicating that the answer was false and 1 indicating that the answer was right and sensitivity ( $\left.\mathrm{A}^{\prime}\right)$. $\mathrm{A}^{\prime}$ is calculated as follows: $A^{\prime}=$ $1-1 / 4[f r / c r+(1-c r) /(1-f r)]$, with $\mathrm{fr}=$ falsely recognized words and $\mathrm{cr}=$ correctly recognized words. The electrophysiological dependent measures were the amplitude and latency of the P150, N200, P3a and P3b ERP components.

\section{Electroencephalography}

The electroencephalogram (EEG) was recorded using 32 electrodes attached to a cap according to the international 10-20 system (Jasper, 1958). The ground electrode was placed on the AFz location, on the forehead. An electrode placed at the left mastoid served as reference. An electrooculogram (EOG) was measured bipolarly vertically above and below the right eye and horizontally next to the right and the left eye.

Data were sampled at $1000 \mathrm{~Hz}$ and filtered online between 0.05 and $100 \mathrm{~Hz}$. Offline, data were screened for artefacts and filtered between 1 and $30 \mathrm{~Hz}$. Event-related potentials were extracted by stimulus locked averaging of epochs from 100 milliseconds before to 1000 milliseconds after stimulus presentation.

For both tasks, ERPs were analysed at $\mathrm{Fz}, \mathrm{FCz}, \mathrm{Cz}, \mathrm{CPz}, \mathrm{Pz}, \mathrm{P} 3$ and $\mathrm{P} 4$. In the ERPs extracted from the EEG during the SMS task, five peaks could be discerned. P150 was determined as the highest amplitude within 110-210 ms after stimulus onset and N200 as the lowest amplitude within 180-300 ms after stimulus onset. P3a and P3b were determined as the highest amplitude within windows individually specified for each participant. The windows for P3a and P3b were not overlapping and had to be between 260 and 430 ms after stimulus onset. In addition, the latencies of these four peaks were included in the analysis.

In the VVLT, the same components were observed. P150 was determined as the highest amplitude within a window of 130-210 ms after stimulus onset, N200 as the lowest amplitude within 180-260 ms after stimulus onset and P3a and P3b were again determined as the highest amplitude within windows individually specified for each participant. The windows for P3a and P3b were chosen such that they would not overlap and fell between 230 and 
$420 \mathrm{~ms}$ after stimulus onset. Latencies of these four peaks were included in the analysis.

\section{Statistical analysis}

All behavioural and ERP data were analyzed by means of repeated measures analysis of variance (ANOVA) with the within subjects factor treatment, which had two levels: PLA and TPD. Additional within subject factors were electrode position (five levels), workload (three levels: 1, 2 and 4 letters memorized) and stimulus type (2 levels: old and new) for the SMS task. For the VVLT additional variables were electrode position (five levels), trial (three levels: trial 1, 2 and 3) and valence (three levels: positive, negative and neutral) for the immediate recall part and stimulus type (two levels: old and new) for the recognition part. Behavioural data, including reaction time and accuracy, and ERP data, including amplitudes and latencies, were tested for main effects and all possible interaction effects. The effects were evaluated using the Greenhouse-Geisser correction and were considered significant at $\mathrm{p}<.05$. Where necessary the Bonferroni correction was applied. In case of a significant main effect, posthoc pairwise comparisons were conducted. Interaction effects were analyzed post hoc by conducting additional repeated measures ANOVAs, including only those factors that were involved in the interaction.

\section{Results}

\section{Blood samples}

After TPD, plasma tyrosine and phenylalanine level was significantly reduced by $57 \%$ at $T 7$, compared to $\mathrm{T0}\left(\mathrm{F}_{1,13}=131.161, \mathrm{p}<.001\right)$ and the tyrosine and phenylalanine/ $\Sigma$ LNAA ratio was reduced by $62 \%$ at $T 7\left(F_{1,13}=108.134, p<.001\right)$. Tyrosine and phenylalanine level was increased by $61 \%$ at $\mathrm{T} 7$ compared to baseline $\left(\mathrm{F}_{1,15}=24.150, \mathrm{p}<.001\right)$ in the placebo condition and the tyrosine and phenylalanine/ $\Sigma$ LNAA ratio was increased by $40 \%$ at $T 7\left(\mathrm{~F}_{1,15}=21.992, \mathrm{p}<.001\right)$.

\section{Questionnaires}

Neither the Bond \& Lader Visual Analogue Scale nor the complaint list showed significant differences between the PLA and TPD conditions $\left(F_{s}<1.391\right)$.

\section{Sternberg Memory scanning task}

Task performance

The behavioural measures slope, intercept, reaction time and accuracy, were not affected by the treatments (see Table 1). However, TPD appeared to 
speed up reaction time $\left(F_{1,16}=3.995, p=.063\right)$, although this effect did not reach significance.

Table 1

Mean scores for dependent variables of the SMS task after PLA and TPD

\begin{tabular}{lll}
\hline & Mean (SEM) scores \\
\hline & PLA & TPD \\
Reaction time (ms) & $472(12)$ & $460(11)$ \\
Accuracy & $0.96(.01)$ & $0.96(.01)$ \\
Slope (ms/letter) & $41(3)$ & $37(3)$ \\
Intercept (ms) & $417(11)$ & $410(9)$ \\
\hline
\end{tabular}

To evaluate whether the task worked appropriately, the effects of workload and stimulus type on reaction time and accuracy were analyzed. Reaction time increased as workload increased $\left(F_{1.35,21.64}=202.805, p<.001\right)$ and reaction time was longer for new as compared to old items $\left(F_{1,16}=71.148, p<.001\right)$.

\section{ERP measures}

Figure 1 depicts the grand average ERPs per treatment condition and task manipulation at $\mathrm{Cz}$. The ERP latencies and amplitudes were analyzed using repeated measures ANOVA with treatment, electrode position, workload and stimulus type as within subjects factors. Inspection of the main effects of workload and stimulus type learned that the task produced the expected effects on the ERPs. For instance, P3a amplitude decreased with increasing workload $\left(F_{1.56,24.94}=20.790, p<.001\right)$.
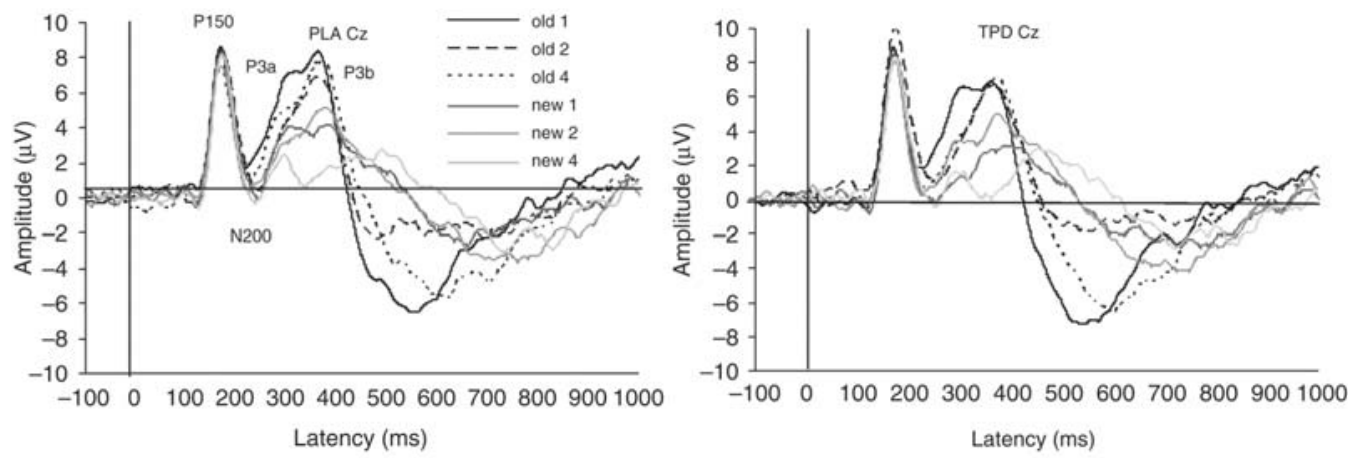

Figure 1. Grand average ERPs during the SMS task, corresponding to the six combinations of workload and stimulus types (workload 1, 2 and 4 and old versus new stimuli), for Cz. Treatment conditions are depicted in separate plots (left: PLA, right: TPD). 
The relationship between treatment and amplitude and latency of the ERP components is plotted in Figure 2. Treatment affected amplitude and latency of several components. There was no significant main effect of the treatment on P150 amplitude or latency. N200 was delayed after TPD $\left(F_{1,16}=4.911\right.$, $\mathrm{p}<.05)$, as compared to PLA. TPD decreased P3a amplitude, as compared to $\operatorname{PLA}\left(\mathrm{F}_{1,16}=5.715, \mathrm{p}<.05\right)$. Furthermore analysis of the P3a latency revealed an interaction between treatment, workload and stimulus type $\left(F_{1.97,31.56}=6.473\right.$, $\mathrm{p}<.01$ ). Post hoc analysis did not reveal any significant effects of treatment. For P3b latency, an interaction between treatment and electrode position was found $\left(F_{2.41,38.53}=3.398, p<.05\right)$. Post hoc analysis revealed that at the $F z$ electrode, P3b latency was increased by TPD $\left(F_{1,16}=5.229, p<.05\right)$.

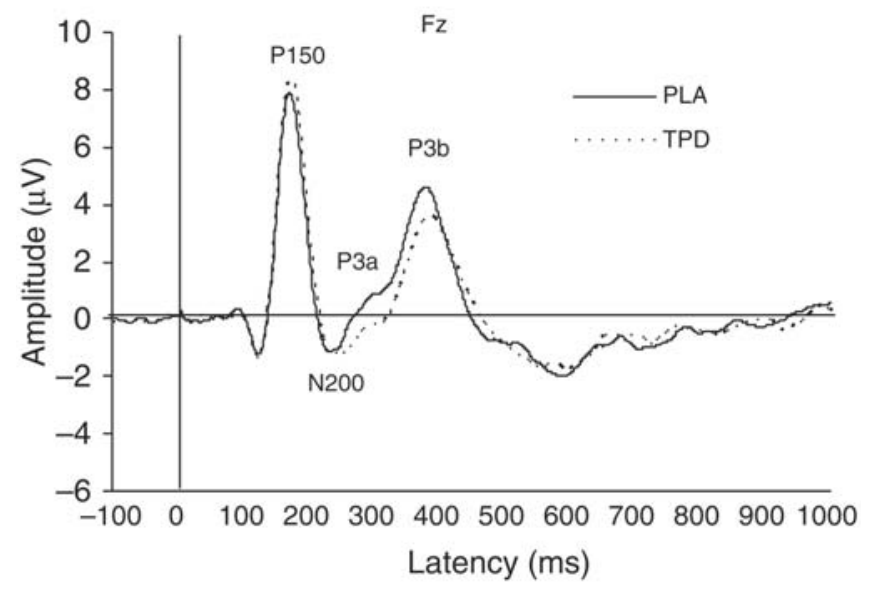

Figure 2. Grand average ERPs during the SMS task after PLA and TPD at Fz.

\section{Visual Verbal Learning Test}

\section{Task performance}

Immediate recall scores and delayed recall scores are listed in Table 2.

The hit rate in the immediate recall phase of the VVLT was not affected by the treatment. However, TPD reduced false alarms and doubles (false alarm: $F_{1,16}=6.272, p<.05 ;$ doubles: $F_{1,16}=7.385, p<.05$ ). Hit rate significantly increased over trials $\left(F_{1.67,26.75}=170.913, p<.01\right)$, as should be expected. Neither delayed recall (see Table 2, $F_{s}<1.595$ for hits, false alarms and doubles) nor recognition (see Table 3, $F_{s}<1.358$ for accuracy, RT and A') were affected by the treatment. However, accuracy was lower when an old item was presented compared to when a new item was presented in the recognition test $\left(F_{1,16}=7.264, p<.05\right)$. Valence of the words did not affect memory performance. 
Means of percentages of hits, false alarms and doubles of the VVLT after PLA and TPD

\begin{tabular}{|c|c|c|c|c|c|c|}
\hline & \multicolumn{6}{|c|}{ Mean (SEM) of percentages of words recalled } \\
\hline & PLA & & & TPD & & \\
\hline & Hits & False alarms & Doubles & Hits & False alarms & Doubles \\
\hline immediate recall & $55.6(2.4)$ & $1.7(.5)$ & $4.6(.8)$ & $52.9(2.9)$ & $0.7(.2)$ & $2.5(.4)$ \\
\hline delayed recall & $61.6(3.7)$ & $2.4(.8)$ & $2.9(.7)$ & $57.3(4.3)$ & $1.8(.6)$ & $1.8(.6)$ \\
\hline Immediate recall-postive words & $56.9(3.2)$ & & $3.8(1.1)$ & $53.6(2.8)$ & & $1.8(.5)$ \\
\hline immediate recall-negative words & $53.8(3.1)$ & & $4.1(.8)$ & $52.6(3.7)$ & & $3.1(.7)$ \\
\hline immediate recall-neutral words & $57.2(3.6)$ & & $5.6(1.3)$ & $52.0(4.2)$ & & $2.6(1.4)$ \\
\hline delayed recall-postive words & $20.3(1.6)$ & & $.7(.4)$ & $19.6(1.3)$ & & $1.1(.3)$ \\
\hline delayed recall-negative words & $19.8(1.3)$ & & $1.1(.4)$ & $18.3(1.8)$ & & $.3(.2)$ \\
\hline delayed recall-neutral words & $22.5(1.7)$ & & $1.0(.7)$ & $19.6(2.0)$ & & $.0(.0)$ \\
\hline
\end{tabular}

Table 3

Mean reaction time, accuracy and sensitivity of the VVLT, recognition test after PLA and TPD

\begin{tabular}{lll}
\hline & \multicolumn{2}{l}{ Mean (SEM) scores } \\
\hline & PLA & TPD \\
Reaction time (ms) & $749(23)$ & $738(21)$ \\
Accuracy & $0.91(.02)$ & $0.89(.02)$ \\
Sensitivity (A') & $0.95(.01)$ & $0.94(.01)$ \\
\hline
\end{tabular}




\section{ERP measures}

Grand average ERPs, averaged over trials, after PLA and TPD are shown in Figure 3. A repeated measures ANOVA with treatment and electrode position as within subjects factors was run on these data. P150 latency was increased after TPD compared to PLA $\left(\mathrm{F}_{1,16}=6.610, \mathrm{p}<.05\right)$. For $\mathrm{N} 200$ amplitude an interaction between treatment and electrode position was found $\left(F_{1.92,30.66}=5.448, p<.05\right)$. Post hoc analysis revealed that the amplitude was different over different electrode positions for the placebo condition, but there was no clear pattern. P3b latency was delayed after TPD as compared to PLA $\left(F_{1,16}=6.398, p<.05\right)$. Furthermore, there was an interaction between treatment and electrode position $\left(\mathrm{F}_{2.32,40.37}=2.788, \mathrm{p}<.05\right)$. Post hoc analysis showed that the latency increasing effect of TPD was significant on $\mathrm{FCz}$ and $\mathrm{Cz}$ electrode positions.

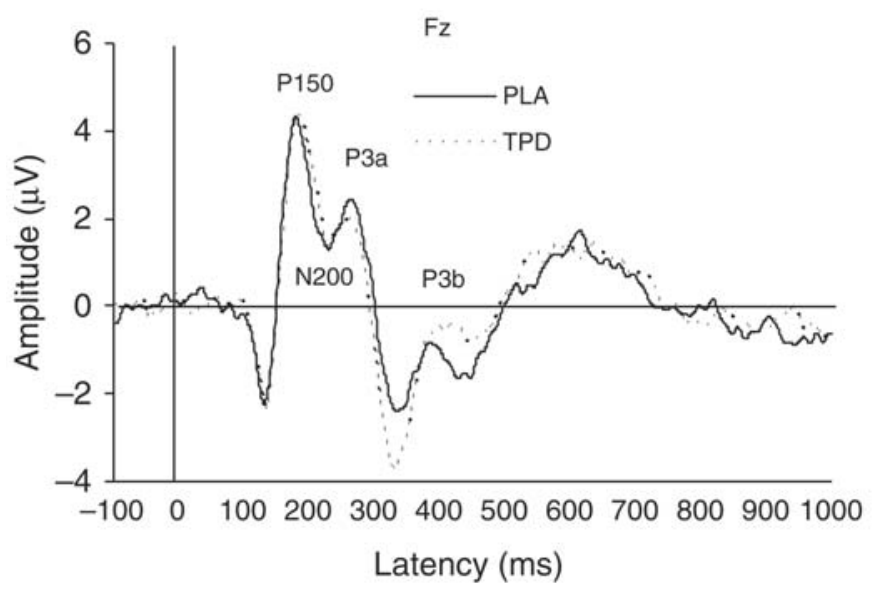

Figure 3. Grand averages during the encoding phase of the VVLT after PLA and TPD at Fz.

Figure 4 depicts grand average ERPs measured during recognition testing after PLA and TPD. ERP amplitudes and latencies were again analyzed using a repeated measures ANOVA with treatment (PLA and TPD) and stimulus type (old and new) as within subjects factor. This analysis revealed that P150 was not altered by TPD. Nor were any main effects of treatment observed for the N200 component. As for the latency data, however, an interaction between treatment and stimulus type was found $\left(F_{1,16}=8.278, p<.05\right)$, which indicated that N200 latency decreased after TPD when an old item was presented $\mathrm{P} 3 a$ latency was decreased after TPD as compared to PLA $\left(\mathrm{F}_{1,16}=6.868, \mathrm{p}<.05\right)$. 


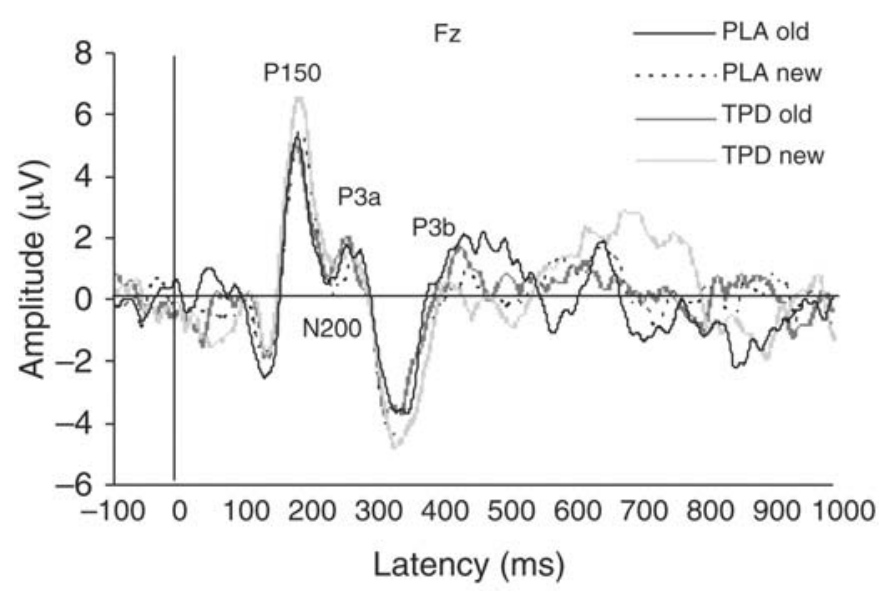

Figure 4. Grand average ERPs during the recognition test of the VVLT after PLA and TPD at presentation of old and new items at Fz,.

\section{Discussion}

This is the first study that investigated the effects of dopamine depletion on working memory and episodic memory, as well as their electrophysiological correlates. After TPD, the tyrosine and phenylalanine/ $L$ LNAA ratio was decreased by $62 \%$. This is a significant level of depletion and similar to previous studies using the same method (Grevet, et al., 2002; Harrison, et al., 2004).

TPD did not significantly affect working memory performance but did have some effects on episodic memory, as was indicated by a decreased false alarm rate and a decrease in the double mentioning of words. During the performance of a working memory task, TPD delayed N200 latency, decreased P3a amplitude and increased P3b latency.

After TPD, both P150 and P3b latency were increased during episodic memory encoding. During the recognition phase, TPD decreased P3a latency. Accordingly, the hypothesis that TPD would interfere with working memory was not confirmed by the behavioural data. The hypothesis that TPD would negatively affect episodic memory was even disconfirmed by the behavioural data, since fewer false alarms and doubles were observed after TPD. On the other hand effects observed on ERPs suggest that there was a memory modulating effect of TPD. It is suggested that these effects were too small to be detected in the behavioural data.

Previous studies showed conflicting results with respect to the effect of TPD on working memory (Ellis, et al., 2007; Gijsman, et al., 2002; Harmer, et al., 2001; Harrison, et al., 2004; Lythe, et al., 2005; Mehta, Gumaste, et 
al., 2005; Nathan, et al., 2002). A satisfying explanation for this inconsistency has not yet been given. In this study, the lack of behavioural effects could not be attributed to a lack of depletion. Furthermore, both behavioral and ERP measures of the working memory task showed the expected variations between the different task conditions (Pelosi, Hayward, \& Blumhardt, 1995). It is important to note that the placebo condition in this study involves the administration of a balanced amino acid mixture, which led to a $40 \%$ increase in the tyrosine and phenylalanine/ LLNAA ratio. It is not clear how this may have affected the results, but for future studies inclusion of baseline testing may be considered.

Furthermore, it is important to note that previous studies used other working memory tests than the one used in the present study. It is highly likely that dopamine has differential effects on different forms of working memory. However, even among studies using comparable tasks, there were some that found an effect of TPD, whereas others did not. It has therefore been proposed that although TPD does alter dopaminergic transmission, its effect may not be large enough to consistently alter neuropsychological function (Lythe, et al., 2005). With respect to the present data, this may be a valid suggestion, since we found no effects on behavioural measures, even though changes in the ERPs were observed.

The effects of TPD on ERP measures indicate that TPD altered brain processes, which are possibly related to memory function. TPD delayed N200 latency. N200 amplitude has been linked to response inhibition or -conflict (Bokura, Yamaguchi, \& Kobayashi, 2001; Falkenstein, Koshlykova, Kiroj, Hoormann, \& Hohnsbein, 1995; Forster \& Pavone, 2008). Increased latency possibly reflects prolonged duration or delayed onset of response conflict. Alternatively, the current finding may indicate increased response selection time after depletion of tyrosine and phenylalanine, since N200 latency has also previously been related to the timing of response selection (Gajewski, Stoerig, \& Falkenstein, 2008).

TPD decreased P3a amplitude. P3a is considered a novelty related peak, where an increased amplitude most likely reflects an alerting effect of the novel stimulus (Coull, 1998). Decreased P3a amplitude, therefore, may indicate decreased alertness. This may be attributed either to a decrease in dopamine levels after TPD or an increase of dopamine levels after the placebo condition. Indeed, Grevet et al. (2002) reported increased subjective alertness ratings after consumption of the balanced amino acid mixture as compared to baseline. P3b latency was increased by TPD. Since P3b latency is known to increase when categorization of the stimulus is made more difficult by degrading the stimulus or when memory set size increases (Garcia-Larrea \& 
Cezanne-Bert, 1998; Smulders, Kok, Kenemans, \& Bashore, 1995), it can be inferred that the stimulus categorization effect was increased by TPD.

Together, the described effects suggest that TPD affected stimulus processing during working memory. However, since no effects were observed on behavioural measures it is suggested that effects of TPD are not large enough to affect performance. This idea is supported by findings from other studies (Ellis, et al., 2007; Mehta, Gumaste, et al., 2005).

Episodic memory was not impaired by TPD. However, after TPD fewer false alarms and doubles were observed in the immediate recall phase. A decrease in false alarms has also been observed after 5-HT suppletion, whereas false alarms have been shown to increase after serotonin depletion (Meeter, Talamini, Schmitt, \& Riedel, 2006). These contrasting effects are in line with the idea that dopamine and serotonin have opposing influences on working memory (Luciana, Collins, \& Depue, 1998). Hit rate and false alarm rate (taking together false alarms and doubles) were combined post hoc in a sensitivity measure ( $\left.A^{\prime}\right)$ using the same formula as used in the recognition test. This measure indicated an increase in sensitivity after TPD $\left(A^{\prime}=.572\right.$ in the placebo condition and $A^{\prime}=.753$ after TPD). This further supports the idea that dopamine and serotonin have opposing influences, since sensitivity has been observed to decrease after serotonin depletion (Schmitt et al., 2000).

The finding that TPD did not negatively affect performance on the word learning test is at odds with the findings of Grevet et al. (2002), who found decreased performance on delayed recall as assessed in Rey's Auditory Verbal Learning Test (RAVLT) after TPD. However, our findings are consistent with those of Harrison et al. (2004), who found no effect of TPD on a 15 word visual verbal learning test. Although this has not been tested explicitly it may be possible that TPD exerts larger effects on auditory word learning compared to visual word learning.

On the recognition task there was no effect of TPD. The lack of memory impairing effects of TPD could not be explained by ineffectiveness of the task, since, as expected, hit rate in the immediate recall test increased over trials, and accuracy on the recognition task was lower for old, as compared to new items.

It was expected that TPD and placebo would differentially affect responses to positive and negative words (McLean, et al., 2004; VrshekSchallhorn, et al., 2006). However, this was not the case. Perhaps differences between positive and negative words can only be observed when a response has to be made immediately after appearance of the stimulus (as is the case in the Go/No-Go task (McLean, et al., 2004) and not when there is a delay between stimulus presentation and testing. 
ERPs recorded during the encoding phase of this episodic memory task revealed that TPD delayed the latency of both P150 and P3b ERP components. $\mathrm{P} 150$ is, in addition to working memory, also thought to be related to stimulus categorization (Schendan, Ganis, \& Kutas, 1998). Although in the VVLT no categorization is necessary, encoding may be regarded similarly to categorization. Delayed P150 latency may reflect a delay in this process. This is in line with the effect on P3b latency, since P3b is known to be dependent on stimulus categorization time (Luck, 2005), or in this case, encoding time. A delay in encoding therefore may lead to a delay in P3b latency.

During recognition testing, P3a latency was decreased by TPD. As explained above, P3a amplitude is thought to be related to alertness or novelty (Coull, 1998). An effect on latency is therefore more difficult to explain.

In summary, TPD did not affect episodic memory performance. Participants even produced fewer false alarms and reported fewer words two times in the immediate recall phase. On the ERPs, however, some effects were observed that were indicative of adverse effects of TPD on memory. Since an effect of TPD on word learning was found previously (Grevet, et al., 2002) and electrophysiological correlates of memory were affected by TPD in the present study, it is suggested that effects of TPD did affect memory processing but effects on memory performance remained sub threshold.

In conclusion, the current findings suggest that TPD, which lowers dopamine levels, affects memory related processing. Dopamine is likely to be involved both in working memory as well as episodic memory. This has implications for the study of memory. Where previously the focus has been on other neurotransmitters such as acetylcholine and serotonin, it has now become clear that dopamine also deserves attention. Future studies may further improve the study design in order to produce more sizeable effects. 
Part 2: The role of dopamine in Psychomotor function 

Chapter 5. Higher, faster stronger: The effect of dynamic stimuli on response preparation and CNV amplitude

In revision

\section{Abstract}

The Contingent Negative Variation (CNV) is a slow negative shift in the electroencephalogram (EEG), observed during response preparation. To optimalize the CNV paradigm, this study developed a task using dynamic stimuli and next combined this task with a Go/No-go test. In the first experiment, 19 healthy volunteers were subjected to the classic Traffic light (TL) task and the new dynamic Lines task. In the Lines task, response time was faster and CNV amplitude was larger compared to the TL task. In the second experiment, 20 healthy participants were tested on a Go/No-go version of the Lines task. Response times increased as the probability of response requirement decreased. CNV amplitude was larger when probability of response requirement was higher. In conclusion, the dynamic task promotes response preparation. The new tasks may be especially valuable in groups with attention difficulties (i.e. elderly or ADHD patients). 


\section{Introduction}

The Contingent Negative Variation (CNV) is a slow negative shift in the electroencephalogram (EEG) which can be observed during response preparation to an anticipated stimulus (Bares, Rektor, Kanovsky, \& Streitova, 2003; Tecce, 1972; van Boxtel \& Brunia, 1994). Since its' discovery by Walter in 1964 it has been studied extensively (Walter, Cooper, Aldridge, McCallum, \& Winter, 1964). The amplitude of the CNV has been shown to increase with increased motivation and attention (McCallum, Cooper, \& Pocock, 1988; Tecce, 1972). The diversity of tasks that are employed to evoke the CNV reflects the variety of applications of the CNV, ranging from fundamental research to studies in different clinical groups. The CNV may be affected in patients with attention deficit/hyperactivity disorder (ADHD), Alzheimer's Disease (AD) and Parkinson's disease (PD) and can also be used to evaluate drug effects (Amabile, et al., 1986; Kratz, et al., 2009; Linssen, et al., 2011; Oishi, et al., 1995; van Deursen, Vuurman, Smits, Verhey, \& Riedel, 2009). Hence, the CNV could potentially be used to screen for normalization of CNV amplitude in neuropsychiatric patients in response to treatment. The current paper describes the development of instruments that could eventually be applied as a screening tool, seeking to implement previously acquired knowledge about the CNV to optimize the CNV paradigm and reliably produce large CNVs. To this end, new response preparation tasks were designed to promote motivation and attention during task performance and induce larger CNVs and faster responses compared to conventional response preparation tasks.

Two components can be distinguished in the $\mathrm{CNV}$, an early and a late component or wave, or the 'O-wave' and ' $\mathrm{E}$-wave', referring to orientation and expectancy (Klorman \& Bentsen, 1975; Loveless \& Sanford, 1974b). The early wave increases with stimulus intensity, is enhanced when auditory warning stimuli are used, and habituates over trials, all of which support that it reflects an orienting response (Gaillard, 1976; Loveless \& Sanford, 1975; Weerts \& Lang, 1973). It has been proposed that the late CNV is a composite of a readiness potential and stimulus preceding negativity (Brunia, 1988; van Boxtel \& Brunia, 1994), reflecting motor preparation and stimulus anticipation (Brunia \& van Boxtel, 2001).

The early CNV has its cortical origin in prefrontal supplementary sensorimotor areas; the late wave in prefrontal, supplementary sensorimotor, primary somatosensory, primary motor, temporal and occipital areas (Babiloni, et al., 2005). Hence, early CNV has been found to be least prominent at the $\mathrm{Pz}$ electrode location whereas the late wave was hardly visible at the Fz electrode (Haagh \& Brunia, 1985; Klorman \& Bentsen, 1975). 
The classic CNV task constitutes a warning stimulus (S1) followed by an imperative stimulus (S2) after a fixed and known interval. Stimulus manipulations differentially affect the early and late CNV waves (Y. Nagai, et al., 2004). For example, the amplitude of the early CNV wave increases with increased warning signal duration (Klorman \& Bentsen, 1975), whereas the amplitude of the late wave increases if the imperative stimulus contains information about the required response (van Boxtel \& Brunia, 1994). In a Go/No-go paradigm, in which the warning stimulus gives information about the required response, the warning stimulus may affect both early and late CNV (Babiloni, et al., 2004; J. L. Smith, Johnstone, \& Barry, 2006, 2007).

In order for the response preparation task to be used as a screening tool, it needs to reliably evoke a CNV. Since CNV amplitude has been reported to be decreased in clinical groups such as PD and ADHD patients (Banaschewski \& Brandeis, 2007; Oishi, et al., 1995), a successful task should induce large CNV waves. To acquire an optimal CNV, a substantial number of trials have to be recorded in which the subjects have to produce speeded responses. Furthermore, the interval between $\mathrm{S} 1$ and $\mathrm{S} 2$ needs to be rather long ( 3 to 4 seconds) in order to observe both an early and a late wave (Fan, et al., 2007). This is potentially problematic, as especially the elderly, children, $A D$ and $A D H D$ patients may suffer from loss of focused attention and possibly fail to show an anticipatory response (Loveless \& Sanford, 1974a; van Deursen, et al., 2009). In this study, an attempt was made to overcome these problems by developing new tasks using stimuli that promote response anticipation.

In a conventional response preparation task, using static stimuli, there is a 4 second window in which nothing happens and attention is easily diverted away from the task. Therefore, it was decided a new task should employ moving stimuli. We designed a task in which participants view two lines moving towards each other that intersect after 4 seconds, to which the participant has to respond by a button press. In this 'Lines task' participants can continuously track the lines during the interval between S1 (the appearance of the lines) and S2 (the crossing of the lines). A major advantage of this task as opposed to static tasks or dynamic tasks in which the timing of S2 is varied (Jaskowski \& Verleger, 1993) is that the movement of the lines allows very precise estimation of the occurrence of the imperative stimulus which was expected to enhance CNV amplitude and speed responses. An additional advantage of the Lines task could be that participants may feel more encouraged to provide a quick response and hence, maintain focused attention. In experiment 1 , we tested the hypothesis that the 'Lines task' would lead to improved response preparation as indicated by an increase in 
late CNV amplitude and faster response times when compared to a standard CNV task using static stimuli.

Once efficacy of this task was established, the task was further adapted, creating a Go/No-go task. Within a Go/NoGo task, it is uncertain whether a response will be required. We were interested to see how this would affect the CNV. Moreover, in contrast with the S2 in the Lines task, the imperative stimulus in our Go/NoGo task carries information as to whether a response has to be given or not, an aspect that may affect not only the late, but also the early CNV wave. Both early and late CNV wave, as induced by the Go/No-go task were studied in experiment 2.

\section{Experiment 1}

\section{Method}

\section{Participants}

Nineteen healthy male volunteers (mean age $=23.4, \mathrm{SD}=5.4$ ) were selected and paid to participate. They were all right handed. Participants were recruited by means of advertisement posters in university buildings and advertisements in the university newspaper. All subjects gave written informed consent. The study was part of a larger study which was approved by the ethical committee of Maastricht University and conducted in accordance with the Declaration of Helsinki 1975, revised Seoul, 2008.

\section{Procedure}

Participants were invited into the lab at either 8:00 or 8:30 AM. EEG was measured using eleven electrodes placed on the scalp, according to the 10-20 system (Jasper, 1958)at the Fz, F3, F4, Cz, C3, C4, Pz, P3, P4, 01 and 02 electrode positions. Electrodes placed on the left and right mastoid served as reference channels. The ground electrode was placed at the AFz location on the forehead. An electro-oculogram (EOG) was measured bipolarly above and below the right eye and horizontally next the left and right eye. Impedances were kept below $5 \mathrm{kOhm}$. Data were sampled at $1000 \mathrm{~Hz}$ and filtered online between 0.05 and $100 \mathrm{~Hz}$. Participants received task instructions both orally and on the computer screen. They performed two different tasks: first a standard response preparation task, the 'TL task' (static task) immediately followed by the 'Lines task' (dynamic task).

\section{CNV Traffic light task}

In the CNV Traffic light (TL) task, participants watched the computer screen and were presented with a filled red circle (S1) on a black background. After 4 seconds, the red circle turned green (S2). Subjects were instructed to 
respond to the green circle by pressing a button with the index finger of the dominant (right) hand on a table mounted response box as fast as they could. The test consisted of 32 trials.

\section{CNV Lines task}

In the CNV Lines task, S1 was the appearance of two red line drawings. The Lines were oriented at a 90 degree angle of each other and after appearing both moved diagonally upwards to the centre of the screen (forming and inverted V). The full movement of the lines spanned approximately $10 \mathrm{~cm}^{2}$. Participants were instructed to fixate on the center of the screen between trials. This was indicated by a fixation cross, which disappeared at the start of the trial. After 4 seconds, the Lines crossed and turned green (S2). At this moment, participants pressed a response button with the index finger of the dominant (right) hand as fast as possible. The visual angle between the fixation cross and the location where the lines crossed was $0.026^{\circ}$. The test consisted of 25 trials.

\section{Data analysis}

EEG data were screened for artefacts offline, corrected for eye movements (Semlitsch, Anderer, Schuster, \& Presslich, 1986) and a low pass filter of 10 $\mathrm{Hz}$ was applied. Event-related potentials were extracted by stimulus locked averaging of epochs starting 100 milliseconds before S1 and ending 1000 milliseconds after S2. A baseline correction was applied on the whole epoch, using the $100 \mathrm{~ms}$ interval preceding $\mathrm{S} 1$ as reference. Late CNV amplitude was calculated by averaging the amplitude over the last 100 milliseconds before S2. Since the CNV amplitude was expected to be maximal at the central electrodes analyses was restricted to $\mathrm{Cz}, \mathrm{C} 3$ and $\mathrm{C} 4$ electrodes.

Reaction time and EEG data were analyzed using repeated measures analysis of variance (ANOVA) with the within subjects factor task which had two levels, namely the TL task and the Lines task. For the amplitude data there was an additional within subjects factor electrode which had 3 levels $(\mathrm{C} 3, \mathrm{Cz}, \mathrm{C} 4)$. The behavioural dependent variable was response time (in ms) of correct responses (i.e. excluding responses before the lines changed color). The effects were evaluated using Greenhouse-Geisser correction and were considered significant at $p<0.05$. Where necessary, the Bonferroni correction was applied. In the case of a significant main effect, post hoc pairwise comparisons were conducted. Interaction effects were analyzed post hoc by conducting additional repeated measures ANOVAs, including only those factors that were involved in the interaction. Only significant effects are reported. 


\section{Results}

Average response time in the Lines task was faster than in the standard TL task $\left(M_{\mathrm{TL}}=247 \mathrm{~ms}, M_{\text {Lines }}=59 \mathrm{~ms} ; \mathrm{F}_{1.0,0,18.00}=932.752, \mathrm{p}<.001\right)$.

Averaged $\mathrm{CNV}$ waves recorded at the $\mathrm{Cz}$ electrode during performance of the TL task and the Lines task are shown in Figure 1. Mean CNV amplitudes at all electrodes are presented in Table 1. Amplitude of the CNV was significantly affected by both task $\left(\mathrm{F}_{1.00,18.00}=8.653, \mathrm{p}<.05\right)$ and electrode position $\left(\mathrm{F}_{1.77,31.84}=8.648, \mathrm{p}<.001\right)$. Amplitude was larger in the Lines task than in the TL task. Pairwise comparisons for electrode positions indicated that CNV amplitude was largest at $\mathrm{Cz}$, smallest at $\mathrm{C4}$, and intermediate at $\mathrm{C3}$.

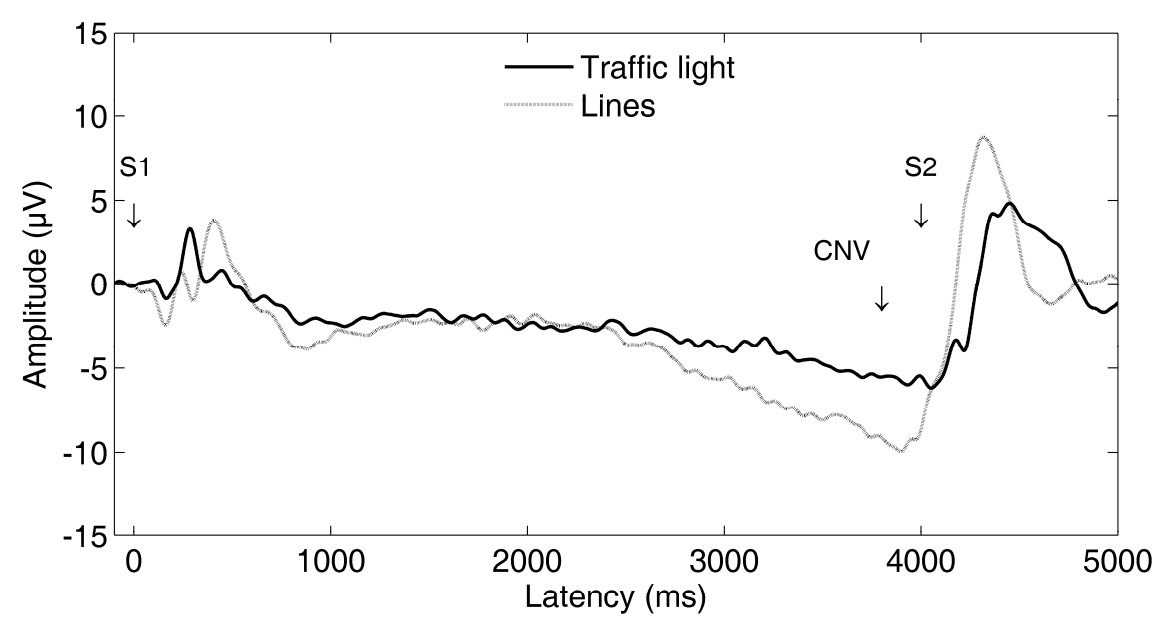

Figure 1. Grand ERPs showing CNV waves measured at Cz during the TL and Lines tasks.

Table 1

Mean amplitudes (SEM) of the CNV for 3 electrode positions of Experiment

\begin{tabular}{lllll}
\hline Electrode & Stoplight & & \multicolumn{2}{l}{ Lines } \\
\hline C3 & -5.12 & $(0.60)$ & -9.29 & $(1.39)$ \\
Cz & -5.79 & $(0.81)$ & -11.07 & $(1.63)$ \\
C4 & -4.62 & $(0.54)$ & -8.60 & $(1.41)$ \\
\hline
\end{tabular}




\section{Experiment 2}

\section{Method}

\section{Participants}

Twenty healthy participants ( 10 male and 10 female, mean age=23.0, SD=1.2) were selected and paid to participate. All but one were right handed. They were recruited by means of advertisement posters at Maastricht University. This study was conducted as a pilot of a larger study which was approved by the ethical committee of Maastricht University and conducted in accordance with the Declaration of Helsinki 1975, revised Seoul, 2008.

\section{Procedure}

Participants were invited into the lab at either 9:00 or 11:00 AM. They signed informed consent and filled out a brief questionnaire assessing demographic variables. Next, they were prepared for EEG measurement. An electrode cap was placed which contained 32 electrodes at locations in accordance with the international 10-20 system (Jasper, 1958). Otherwise, EEG measurement procedures were identical to those in experiment 1.

Participants received task instructions both orally and on the computer screen. The task was carried out in two identical parts each containing 144 trials and lasting approximately 18 minutes, with a 5 minute break in between. The warning stimulus, S1, was the appearance of two red line drawings at the bottom left and right hand corner. The Lines were oriented at a 90 degree angle of each other and after appearing both moved diagonally upwards to the centre of the screen (forming and inverted V). The full movement of the lines spanned approximately $10 \mathrm{~cm}^{2}$. Participants were instructed to focus on the fixation cross at the center of the screen. After 4 seconds, the Lines crossed (S2). At this moment, participants pressed a response button with the index finger of the dominant (right) hand as fast as possible, but only if the Lines turned green (Go trial) at the moment they crossed. If the Lines remained red, participants should withhold the response (No-go trial). The chance that a trial was a Go trial was indicated at S1 by a percentage and could be either 20,50 or $100 \%$ (randomized per block). For each of these percentages, there were 36 Go trials. Consequently, a total of $36100 \%$ trials was presented, whereas for the $50 \%$ trials, there were 72 trials ( 36 go and 36 No-go) and for the $20 \%$ trials, 180 trials were present ( 36 go and 144 No-go). 


\section{Data analysis}

EEG data were screened for artefacts offline corrected for eye movements (Semlitsch, et al., 1986) and a low pass filter of $10 \mathrm{~Hz}$ was applied. Eventrelated potentials were extracted by stimulus locked averaging of epochs starting 100 milliseconds before S1 and ending 4800 milliseconds after S2. A baseline correction was applied on the whole epoch, using the $100 \mathrm{~ms}$ interval preceding S1 as reference. Early CNV was determined as the average amplitude between 750 and $1200 \mathrm{~ms}$ after S1 relative to baseline. Late CNV was determined as the average amplitude between 3600 and $4000 \mathrm{~ms}$ after S1. For both waves, mean amplitudes were analysed for F3, Fz, F4, FC3, FCz, $\mathrm{FC} 4, \mathrm{C} 3, \mathrm{Cz}$ and $\mathrm{C} 4$. Only correct trials were taken into account.

Behavioural and EEG data were analyzed using repeated measures analysis of variance (ANOVA) with the within subjects factor trial type which had three levels, namely the 20,50 and $100 \%$ trials. For the amplitude data there was an additional within subjects factor electrode which had 9 levels (F3, Fz, F4, FC3, FCz, FC4, C3, Cz, C4). Behavioural dependent variables were response time (in $\mathrm{ms}$ ) of correct responses (i.e. excluding prepotent responses and responses to No-go trials), hit rate, and false alarm rate. As in experiment 1, the effects were evaluated using Greenhouse-Geisser correction and were considered significant at $p<0.05$. Where necessary, the Bonferroni correction was applied. In the case of a significant main effect, post hoc pairwise comparisons were conducted. Interaction effects were analyzed post hoc by conducting additional repeated measures ANOVAs, including only those factors that were involved in the interaction. Only significant effects are reported.

\section{Results}

Task performance

Means and standard errors of measurement for the main outcome variables including response time, hit rate and false alarm rate for each trial type are presented in Table 2. There was a significant main effect of trial type on response time $\left(\mathrm{F}_{1.11,21.24}=357.243, \mathrm{p}<.001\right)$. Pairwise comparisons revealed that response times increased as the probability of response requirement decreased. In other words, the more unsure participants were about whether they would have to respond, the slower they responded. Hit rate was smaller in the $100 \%$ Go trials compared to the 20 and $50 \%$ Go trials, due to premature responding in the $100 \%$ Go trials $\left(F_{2,38}=35.119, p<.001\right)$. False alarm rate was larger for the $50 \%$ No-go trials than for $20 \%$ No-go trials $\left(F_{1,19}=9.824, p<.01\right)$. 
Table 2

Mean (SEM) response times, hit rate and false alarm rate for Experiment 2

\begin{tabular}{llllllllll}
\hline & $100 \%$ Go & $50 \%$ Go & $50 \%$ No-go & $20 \%$ Go & $20 \%$ No-go \\
\hline Response time & $103^{*}$ & $(10)$ & $286^{*}$ & $(4)$ & & & $301^{*}$ & $(5)$ & \\
Hit rate & $0.93^{*}$ & $(0.01)$ & 1.00 & $(0.00)$ & & 1.00 & $(0.00)$ & & \\
False alarm rate & & & & & $0.024^{*}$ & $(0.006)$ & & $0.007^{*}$ & $(0.002)$ \\
\hline
\end{tabular}

Amplitude data

Averaged CNV waves recorded at the $\mathrm{Cz}$ electrode during performance of the Go/ No-go task are shown in Figure 2. Mean early and late CNV amplitudes at all electrodes are presented in Tables 3 and 4 respectively.

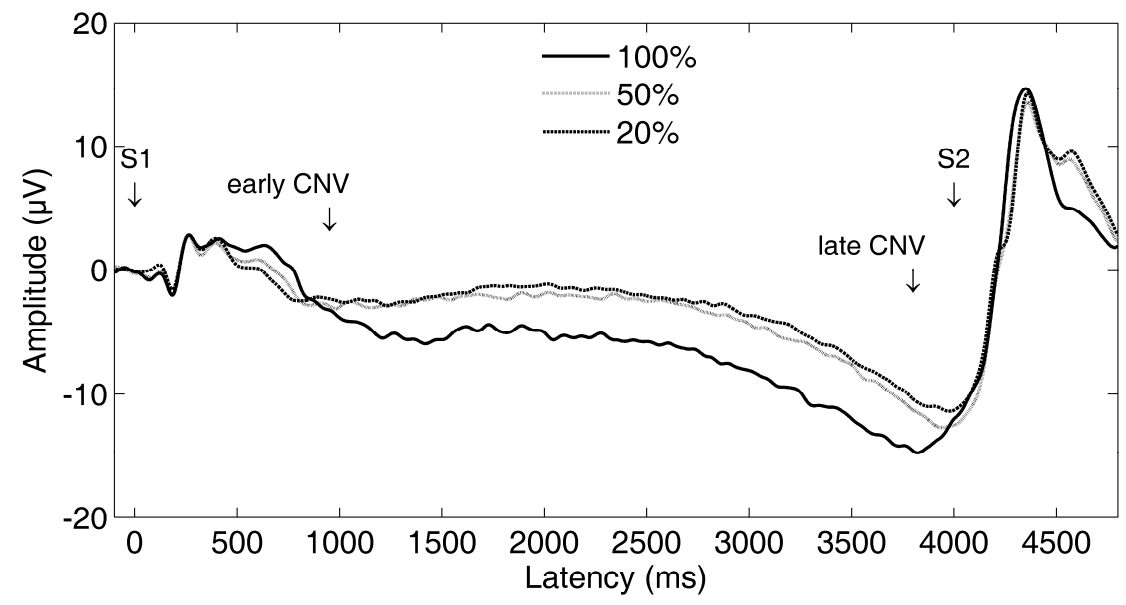

Figure 2. Grand ERPs showing CNV waves measured at $\mathrm{Cz}$ during each of the three trial types with $100 \%, 50 \%$ and $20 \%$ chance on a go trial.

\section{Early CNV wave}

A main effect of electrode position $\left(F_{1.52,28.88}=4.685, p<.05\right)$ indicated that the amplitude of the early CNV differed between electrode positions. Pairwise comparisons revealed that amplitude was larger at $\mathrm{FCz}$ compared to $\mathrm{F} 4$ and $\mathrm{C} 4$, and larger at $\mathrm{Cz}$ than at $\mathrm{C} 4$. When amplitudes were analyzed for each electrode separately, a main effect of trial type was found for the frontal electrodes $\left(F 3: F_{2,38}=4.801, p<.05, F z: F_{2,38}=7.720, p<.01, F 4: F_{1.33,25.19}=4.617\right.$, $\mathrm{p}<.05)$ and $\mathrm{FC} 3\left(\mathrm{~F}_{1.53,29.13}=3.801, \mathrm{p}<.05\right)$ but not for the other 5 electrodes. Pairwise comparisons showed that the amplitude was larger when requirement of response probability was $100 \%$ compared to $50 \%$ at $\mathrm{F} 3, \mathrm{Fz}$ and 
F4 and compared to $20 \%$ at Fz. For FC3 pairwise comparisons revealed no significant results.

Table 3

Mean amplitudes (SEM) of the early CNV for the different trial types in the 9 electrode positions

\begin{tabular}{lllllllll}
\hline Electrode & $\mathrm{F}$ & $\mathrm{df}$ & $100 \%$ & & $50 \%$ & \multicolumn{3}{c}{$20 \%$} \\
\hline F3 & $4.801^{*}$ & $1.78,33.75$ & -2.80 & $(1.03)$ & -1.35 & $(1.01)$ & -1.45 & $(0.75)$ \\
Fz & $7.720^{*}$ & $1.56,29.62$ & -3.94 & $(0.78)$ & -2.28 & $(0.73)$ & -1.89 & $(0.68)$ \\
F4 & $4.617^{*}$ & $1.33,26.19$ & -1.64 & $(0.73)$ & -0.07 & $(0.72)$ & -0.01 & $(0.84)$ \\
FC3 & $3.801^{*}$ & $1.53,29.13$ & -3.61 & $(1.01)$ & -2.33 & $(0.88)$ & -2.21 & $(0.66)$ \\
FCz & 2.206 & $1.35,25.67$ & -1.93 & $(0.82)$ & -0.99 & $(0.75)$ & -0.57 & $(0.80)$ \\
FC4 & 3.375 & $1.55,29.40$ & -4.29 & $(0.95)$ & -3.07 & $(0.86)$ & -2.56 & $(0.71)$ \\
C3 & 1.126 & $1.46,27.65$ & -2.77 & $(0.98)$ & -1.96 & $(0.78)$ & -1.93 & $(0.54)$ \\
Cz & 1.180 & $1.52,28.90$ & -3.54 & $(0.95)$ & -2.83 & $(0.82)$ & -2.42 & $(0.63)$ \\
C4 & 0.512 & $1.42,26.89$ & -1.31 & $(0.81)$ & -0.89 & $(0.68)$ & -0.61 & $(0.67)$ \\
\hline
\end{tabular}

${ }^{*} p<.05$

\section{Late CNV wave}

Amplitude of the late CNV was significantly affected by both trial type ( $F$ $1.49,28.25=4.638, \mathrm{p}<.05)$ and electrode position $\left(\mathrm{F}_{2.64,50.15}=21.012, \mathrm{p}<.001\right)$. In addition, an interaction between these two factors was observed $\left(F_{4.79,90.95}=2.604, \quad p<05\right)$. Pairwise comparisons revealed no significant differences for the trial types. For the electrode positions, pairwise comparisons indicated that the late CNV amplitude generally was smallest for the most frontal electrodes and increased as the position was more central. However, not all pairwise comparisons were significant.

To further explore the interaction between electrode position and trial type, the effect of trial type was analyzed for each electrode position separately (see Table 4). It was shown that amplitude increased with increasing probability of response requirement at $\mathrm{Cz}, \mathrm{C} 3, \mathrm{C} 4, \mathrm{FCz}, \mathrm{FC} 3$ and FC4 and F4 (although this was only significant for the $100 \%$ vs. the $20 \%$ trials). This effect was most significant at the central electrode positions, less significant for the fronto-central positions and non-significant for two out of three frontal positions.

In order to see if the late CNV was lateralized, a comparison was made between electrode positions that were contralateral vs. ipsilateral to the response finger. This revealed a trend suggesting that the late CNV was slightly larger at the central electrode contralateral to the response finger (C3 
in most participants) compared to ipsilateral central electrode (C4 in most participants, $\left.\mathrm{F}_{1,19}=3.409, \mathrm{p}<.10\right)$.

Table 4

Mean amplitudes (SEM) of the late CNV for the different trial types in the 9 electrode positions

\begin{tabular}{lllllllll}
\hline Electrode & $\mathrm{F}$ & $\mathrm{df}$ & $100 \%$ & \multicolumn{2}{c}{$50 \%$} & \multicolumn{3}{c}{$20 \%$} \\
\hline F3 & .992 & $1.39,26.35$ & -7.39 & $(1.05)$ & -6.39 & $(1.03)$ & -6.20 & $(0.91)$ \\
$\mathrm{Fz}$ & 2.576 & $1.60,30.39$ & -8.14 & $(0.97)$ & -7.15 & $(1.07)$ & -6.18 & $(1.08)$ \\
F4 & $5.365^{*}$ & $1.53,29.09$ & -6.34 & $(1.17)$ & -4.34 & $(0.97)$ & -3.80 & $(0.99)$ \\
FC3 & $3.844^{*}$ & $1.51,28.64$ & -9.73 & $(1.14)$ & -7.88 & $(1.01)$ & -7.58 & $(0.84)$ \\
FCz & $3.736^{*}$ & $1.44,27.27$ & -11.91 & $(1.26)$ & -10.41 & $(1.31)$ & -9.15 & $(1.17)$ \\
FC4 & $5.188^{*}$ & $1.58,30.04$ & -8.37 & $(1.30)$ & -6.75 & $(1.11)$ & -5.63 & $(1.08)$ \\
C3 & $4.486^{*}$ & $1.38,26.28$ & -11.16 & $(1.37)$ & -8.72 & $(1.17)$ & -8.57 & $(0.97)$ \\
Cz & $5.956^{*}$ & $1.35,25.63$ & -13.54 & $(1.51)$ & -11.10 & $(1.21)$ & -9.93 & $(1.16)$ \\
C4 & $7.026^{*}$ & $1.62,30.77$ & -10.06 & $(1.32)$ & -7.84 & $(1.17)$ & -6.97 & $(1.06)$ \\
\hline
\end{tabular}

${ }^{*} \mathrm{p}<.05$

\section{Discussion}

The current study aimed to develop response preparation tasks producing CNVs that could be potentially useful as a screening tool. We showed enhanced response preparation in a newly developed task applying dynamic stimuli in relation to a task with static stimuli, as was indicated by larger CNV amplitude and faster response times. In a second experiment we tested whether this effect persisted if the task was adjusted to a Go/No-go format. The probability that participants had to respond (Go trials) differed between the trials. As predicted this manipulation differentially affected amplitudes of the early and late CNV waves and response times. The smaller the probability of response requirement, the smaller the CNV amplitudes and the slower participants responded.

The finding that participants responded faster in the new task, using dynamic stimuli, compared to the standard task with static stimuli, is in line with our hypothesis. Because the stimuli were steadily and continuously moving towards each other during the trial, timing of the response could be monitored very accurately. It is likely that this extra stimulus feature increased the participants' motivation to respond as quickly as possible and maintain focused attention. This is supported by the increase in CNV amplitude, which has previously been associated with motivation and attention (McCallum, et al., 1988; Tecce, 1972). 
Since the participants in experiment 2 were not the same as those in experiment 1 , reaction times cannot be directly compared between the two experiments. However, it is still striking that although the same stimuli were used in the Go/No-go task, responses were much slower compared to the Lines task. Responses to the 50 and $20 \%$ trials were rather close to those in the TL task. In these 'uncertain' trials additional processing related to the decision to make a response can account for this result. Response times in the $100 \%$ trials were faster compared to the TL task, but slower compared to the Lines task. This is surprising, since the $100 \%$ trials in the Go/No-go task are actually identical to the Lines task. Although participants can be sure that they will have to respond unconditionally, they are approximately twice as slow compared to the Lines task. This may partly be explained by the fact that this was a different group of participants. However, other explanations may be that inhibition in the uncertain trials spreads to the $100 \%$ Go trials or the participants did not trust the instructions. This latter explanation was given by Smith, Johnstone \& Barry (2007) for the fact that participants seemed to prepare to give a response on specific No-go trials.

Within the Go/No-go task, the most prominent differences are observed between 'certain' versus 'uncertain' trials. Nevertheless, results of the $50 \%$ and $20 \%$ No-go trials do indicate that they were processed differently. Responses were slower to $20 \%$ trials compared to the $50 \%$ trials, perhaps indicative of stronger inhibition. In support of this idea, relatively more false alarms were produced in the $50 \%$ trials than in the $20 \%$ trials.

The size of the early CNV was positively related to the probability participants had to produce a response. As expected the task manipulation exerted the largest effect on the early CNV at frontal electrodes (Haagh \& Brunia, 1985; Klorman \& Bentsen, 1975). The late CNV on the other hand was maximal over central electrode positions, which is also in line with previous findings (Haagh \& Brunia, 1985; Klorman \& Bentsen, 1975). The effect of the manipulation was largest at these electrodes. The late CNV seemed to be lateralized to the side contralateral to the response hand, which was expected based on previous findings (Butler \& Glass, 1974; Verleger, Paehge, Kolev, Yordanova, \& Jaskowski, 2006).

In sum, in this study, two new tasks were developed to induce a more optimal CNV wave that could possibly be used as a screening tool in future clinical-/pharmacological studies. The adaptations to the regular task proved to be successful in enhancing response preparation as indicated by higher amplitude and faster response times. The tasks' properties are thought to promote motivation and effort of subjects to optimally prepare for each trial and this makes the task especially valuable in participants who have problems sustaining attention such as elderly participants or ADHD patients. 
Furthermore, varying the probability that a response has to be given affects stimulus processing, response preparation and execution as reflected by the effect on the early CNV wave, the late CNV wave and response times respectively. The Go/No-go CNV task presented here may lead to new insights regarding response preparation and inhibition in both clinical and non-clinical groups on or off medication.

\section{Acknowledgements}

The authors would like to thank Conny Quaedflieg for contributing to the study. 
Chapter 6. Contingent Negative Variation as a dopaminergic biomarker: Evidence from dose-related effects of methylphenidate

Linssen, A. M., Vuurman, E. F., Sambeth, A., Nave, S., Spooren, W., Vargas, G., et al. (2011) Psychopharmacology, 218(3), 533-542

\section{Abstract}

Rationale: The basal ganglia play an important role in motor control, which is dependent on dopaminergic input. Preparation of a motor response has been associated with dopamine release in the basal ganglia, and response readiness may therefore serve as a pharmacodynamic marker of dopamine activity.

Methods: We measured response readiness using the amplitude of the contingent negative variation (CNV), a slow negative shift in the electroencephalogram. The CNV is evoked in a paradigm in which a warning stimulus (S1) signals the occurrence of the imperative stimulus (S2) $4 \mathrm{~s}$ later, to which the participant has to respond. CNV was assessed in healthy volunteers after administration of placebo or 10,20 or $40 \mathrm{mg}$ of methylphenidate, a catecholamine re-uptake blocker which primarily enhances the synaptic concentration of dopamine and to a lesser extent also noradrenaline. In addition, participants filled out two visual analogue scales measuring subjective ratings of mood and alertness: Profile of Mood States and Bond and Lader.

Results: Methylphenidate dose dependently increased CNV amplitude and decreased reaction times. Furthermore, participants reported improved mood, feeling more alert, vigorous and content and less angry and tired after methylphenidate.

Conclusions: These results indicate that dopamine availability increases response readiness as measured by the CNV paradigm. The CNV appears to be a good candidate biomarker for assessing changes in dopaminergic function by treatments that either directly or indirectly target the dopaminergic system. 


\section{Introduction}

Dopamine abnormalities are implicated in neuropsychiatric diseases such as Parkinson's disease, schizophrenia and attention deficit hyperactivity disorder (ADHD; (Iversen \& Iversen, 2007; Nieoullon, 2002), but the precise nature of these disturbances and their treatments remains to be understood. This drives the relevance of dopamine-related research and the value of new tools aiding this research. In the present study, a candidate biomarker of dopamine activity is proposed and validated.

The contingent negative variation (CNV) is a slow negative shift in the EEG that can be observed between a warning signal and an imperative stimulus during a reaction time task. The occurrence of this wave has been associated with expectation, anticipation, orientation, attention, intention to act, motivation and response readiness (Bares, et al., 2003; Tecce, 1972; van Boxtel \& Brunia, 1994; Walter, et al., 1964).

Evidence from studies with Parkinson's disease patients using intracranial electrodes and studies combining EEG/fMRI techniques suggests that the CNV is generated in the basal ganglia (Bares \& Rektor, 2001; Fan, et al., 2007; T. Nagai, et al., 2007; Oishi, et al., 1995; Rektor, et al., 2004; Rektor, et al., 2003). An important neuromodulator within the basal ganglia is dopamine which, consequently, has been considered a possible biochemical mechanism underlying the CNV (Amabile, et al., 1986). Converging evidence suggests that CNV may indeed be modulated by dopamine availability (Amabile, et al., 1986; Kopell, Wittner, Lunde, Wolcott, \& Tinklenberg, 1974; Oishi, et al., 1995; Tecce, 1991; Tecce \& Cole, 1974; Tecce, Cole, \& Savignano-Bowman, 1975). Consequently, changes in CNV may signal alterations of dopamine levels in the brain, and the CNV may thus be a basal ganglia originated marker of dopamine activity.

Research with agents affecting other neurotransmitters such as serotonin, noradrenaline and acetylcholine showed that the influence on the CNV is indirect (through interactions with the dopamine system), absent or dose dependent (Ashton, et al., 1980; Ashton, Millman, Telford, \& Thompson, 1976; Ashton \& Rawlins, 1978; Hansenne, Pitchot, Pinto, Papart, \& Ansseau, 2000; Mulder, Linssen, \& de Geus, 2002). Benzodiazepines decrease CNV amplitude (Ashton, et al., 1976; Papart, Ansseau, \& Timsit-Berthier, 1997; Rockstroh, Elbert, Lutzenberger, \& Altenmuller, 1991).

An early and a late CNV component or 'wave' can be distinguished (Klorman \& Bentsen, 1975). The early wave is thought of as an orientation response (Klorman \& Bentsen, 1975) whereas the late CNV consists of a readiness potential and stimulus preceding negativity (Brunia, 1988; van Boxtel \& Brunia, 1994). The late CNV reflects motor preparation and stimulus 
anticipation (Brunia \& van Boxtel, 2001) and is the wave targeted here. To observe both waves, an interval of $4 \mathrm{~s}$ is used in the current study.

Before the CNV can be successfully applied as a dopaminergic biomarker, its sensitivity needs to be established. It is essential to know both the minimal change in dopamine levels that can be detected by means of the CNV and the dose-response curve of the paradigm. Therefore, this study measured CNV amplitude across a range of doses of a dopamine-enhancing drug. The drug chosen was methylphenidate. Methylphenidate inhibits dopamine re-uptake by blocking the dopamine transporter (DAT) and thus enhances dopamine availability (Volkow, Wang, Fowler, Gatley, et al., 1998), has a wide safe dose range, is generally well tolerated and has been used for similar purposes in previous studies (Volkow, et al., 2008; Volkow, Wang, Fowler, Hitzemann, et al., 1998). Methylphenidate is used to treat ADHD and narcolepsy. The maximum dose in children with ADHD is $60 \mathrm{mg} /$ day. PET research has shown that methylphenidate leads to dose-dependent DAT blockade with a dose of $5 \mathrm{mg}$ producing a $12 \%$ blockade, $10 \mathrm{mg} 40 \%, 20 \mathrm{mg}$ 54\%, $40 \mathrm{mg} \mathrm{72 \%} \mathrm{and} 60 \mathrm{mg} 74 \%$ (Volkow, Wang, Fowler, Gatley, et al., 1998). Hence, therapeutic doses of methylphenidate are likely to block more than $50 \%$ of DAT. $T_{\max }$ is observed $1-3 \mathrm{~h}$ after intake, with a half life of approximately $3 \mathrm{~h}$ (Leonard, et al., 2004; Spencer, et al., 2006).

Participants were treated with 10, 20 and $40 \mathrm{mg}$ of methylphenidate, and in addition to CNV amplitude, task performance and subjective drug effects were also measured. It was expected that CNV amplitude would show a dose-related increase and that reaction time would dose dependently decrease. Based on the PET research cited above and other previous studies with methylphenidate (Cooper, et al., 2005; Hermens, et al., 2007), it was expected that a dose of $10 \mathrm{mg}$ would lead to minimal effects. The largest effects were expected with $40 \mathrm{mg}$ of methylphenidate, whereas $20 \mathrm{mg}$ was expected to induce intermediate effects.

\section{Materials and methods}

\section{Participants}

Nineteen healthy male volunteers (mean age $=23.4, \mathrm{SD}=5.4$, range $=19-37$ ) were selected and paid to participate. Participants were recruited by means of advertisement posters in university buildings and advertisements in the university newspaper. Prescreening occurred using a medical history questionnaire and was followed by medical examination.

The main inclusion criteria were between 18 and 40 years of age, body mass index between 18 and $30 \mathrm{~kg} / \mathrm{m}^{2}$ and right-handed. The main exclusion criteria were history or presence of mental or physical disorders as 
assessed by medical history and anamnesis at inclusion, reported presence of psychiatric diseases in first degree relatives, previous drug dependence, selfreported regular drug use, smoking more than five cigarettes per day and consumption of more than 21 alcohol units per week or more than five caffeine containing drinks per day (average reported daily use <2). In addition, volunteers with polymorphisms of the CES1 gene indicative of being a poor metaboliser of methylphenidate were excluded. One participant was excluded based on this criterion.

All subjects gave written informed consent. The study was carried out in accordance with the declaration of Helsinki and approved by the medical ethical committee of Maastricht University.

\section{Design}

The study was conducted according to a double-blind, placebo-controlled, four-way crossover design. Between the testing days, a period of at least $48 \mathrm{~h}$ elapsed, but generally testing days were scheduled approximately 1 week apart. Each participant received one of four single treatments including placebo (PLA) and 10, 20 and $40 \mathrm{mg}$ methylphenidate (M10, M20 and M40) on each testing day. The order of the treatments was randomized following a Williams Latin square design resulting in four different sequences constructed using SAS programme.

\section{Dependent measures}

\section{EEG measurement}

During two response preparation tasks, EEG was measured using 11 electrodes placed on the scalp, according to the 10-20 system at the Fz, F3, F4, Cz, C3, $\mathrm{C} 4, \mathrm{Pz}, \mathrm{P} 3, \mathrm{P} 4, \mathrm{O} 1$ and $\mathrm{O} 2$ electrode positions. The remaining electrodes consisted of four to register vertical and horizontal eye movements, two on the mastoids for reference and one on the forehead as ground. Data were recorded with a $1,000-\mathrm{Hz}$ sample rate and filtered online between 0.05 and $100 \mathrm{~Hz}$. Offline, data were first screened for artefacts and a low pass filter of $10 \mathrm{~Hz}$ was applied on all data. Next, epochs were extracted from the continuous EEG. Each epoch lasted 5,100 ms, starting $100 \mathrm{~ms}$ before S1 and ending $1,000 \mathrm{~ms}$ after S2. A baseline correction was applied to the whole epoch, using the $100 \mathrm{~ms}$ interval preceding $\mathrm{S} 1$ as reference. Averaging the sequential epochs yielded a S1 stimulus locked event-related potential (ERP). CNV amplitude was calculated by averaging the amplitude over the last $100 \mathrm{~ms}$ before S2. Since the CNV amplitude was expected to be maximal at the central electrodes (Brunia, 1988; Klorman \& Bentsen, 1975; van Boxtel \& Brunia, 1994), Cz, C3 and C4 were of main interest and are reported in the Results section. 
Response preparation tasks

CNV stoplight task

In the CNV stoplight task, participants watched the computer screen and were presented with a filled red circle (S1) on a black background. After $4 \mathrm{~s}$, the red circle turned green (S2). Subjects were instructed to respond to the green circle by pressing a button on a table mounted response box as fast as they could. Each test consisted of 32 trials. Reaction time to the onset of S2 was measured in each trial and averaged over the test.

\section{CNV lines task}

A CNV task with a long preparation time can be boring, and participants may become less motivated to produce speeded responses to the imperative stimulus. This may have a detrimental effect on CNV amplitude. Therefore, a new task involving dynamic stimuli was designed. In the CNV lines task, S1 was the appearance of two red line drawings at the bottom left- and right-hand corner. The lines were oriented at a $90^{\circ}$ angle to each other and after appearing both moved towards the centre of the screen. After $4 \mathrm{~s}$, the lines crossed and turned green (S2). At this moment, participants pressed a response button as fast as possible. In two thirds of the trials, a horizontal yellow bar partially blocked the view of the lines as they moved towards the centre of the screen, but the lines were always visible at the moment of crossing. The yellow bar could be small or large, blocking less or more of the trajectory of the lines as they moved towards the centre. The trials with the small bar are collectively called 'lines 1', those with the large bar 'lines 2' and those without a bar 'lines 3'. Of each type, 25 trials were presented. Reaction time was measured and averaged per trial type. These three variations of the new paradigm and the standard paradigm were compared in order to find out which task led to optimal CNV amplitude.

\section{Subjective measures}

Participants completed two rating scales of subjective effects, the Profile of Mood States (POMS; (MCNair, et al., 1992)) and the Bond and Lader (B\&L) visual analogue scales (Bond \& Lader, 1974). These rating scales involved the presentation of 16 (B\&L) or 32 (POMS) bipolar items presented at the ends of a horizontal line on a computer touch screen. Each item consisted of two words describing mood states. Participants had to score their preference for either of the items in accordance with their mood by marking the line closer to one of the two words. Dependent measures were factor scores on the dimensions Alertness, Contentedness and Calmness (B\&L) and Depression, Anger, Fatigue, Vigour and Tension (POMS). 
Blood plasma values and physiological measures

Blood samples were drawn in order to measure blood levels of the drug and neuroendocrine responses. Dependent variables were changes in levels of methylphenidate, prolactin and cortisol in blood during the testing days. Physiological measures including systolic and diastolic blood pressure and heart rate were also taken.

\section{Procedure}

A training session in which all behavioural tasks were practiced took place within 2 weeks before the first testing day. Participants abstained from alcohol during the last $24 \mathrm{~h}$ prior to each testing day and from caffeine containing drinks during the testing days. On testing days, they were collected at their home and arrived at the lab in fasted condition at either $8.00 \mathrm{am}$ or 8:30 am. Inclusion exclusion criteria were checked, including a drug screen (which tested presence of the following substances in urine: cannabis, opiates, cocaine, methamphetamine, benzodiazepines, amphetamines) and blood alcohol concentration measurement. Next, EEG electrodes and a catheter were placed, and participants were given a standardized meal for breakfast at $80 \mathrm{~min}$ per dose $(t=-80)$. At $t=0$, participants ingested four identically appearing capsules with water that all contained either PLA, M10, M20 or M40. Participants went through seven cycles of testing at $t=-60,30$, $60,120,180,240$ and 300 min relative to dosing. At $t=220$, a standard light lunch was served. Each cycle included a fixed order of the CNV stoplight task, the CNV lines task, POMS, B\&L, registration of heart rate and blood pressure and the drawing of blood samples. After collection of the blood samples, the catheter was cleaned with heparin dissolved in isotonic saline, and blood samples were centrifuged for $10 \mathrm{~min}$ at $4,000 \mathrm{rpm}$. Plasma was stored at $-20^{\circ} \mathrm{C}$ and frozen PK samples were moved to a $-80^{\circ} \mathrm{C}$ freezer. After the last measurement, the EEG electrodes and catheter were removed and participants were transported home by taxi.

\section{Data analysis}

Data were analysed using repeated measures analysis of covariance analysis. In this mixed model, the repeated measures of drug (independent variable) on the dependent variables (mean CNV amplitude, reaction time, scores on POMS and Bond \& Lader, heart rate, blood pressure, cortisol and prolactin levels in blood, $T_{\max }$ and $C_{\max }$ ) were tested using baseline (measurement of the dependent variable at $t-60$ ) as a covariate and period (first, second, third or fourth testing day) as between subjects variable. Since treatment consisted of different doses of the same drug and the lower doses may have no or little effect on amplitude, response time and subjective measures, the likelihood of 
finding a main effect on these measures may be rather small, even though higher doses may have a significant effect. Therefore, main effects of drug were not evaluated for the amplitude and reaction time data and the subjective measures. Instead, a priori planned comparisons between each dose relative to placebo were tested for significance, at $p<0.05$, using LSD correction. This is not the case for blood plasma values and physiological measures, where even small doses of the drug were expected to exert significant effects on heart rate, blood pressure, cortisol and prolactin levels and $T_{\max }$ and $C_{\max }$. Therefore, main effects are reported for these measures at a significance level of $p<0.05$. Post hoc testing using LSD correction was done to find out which doses had an effect.

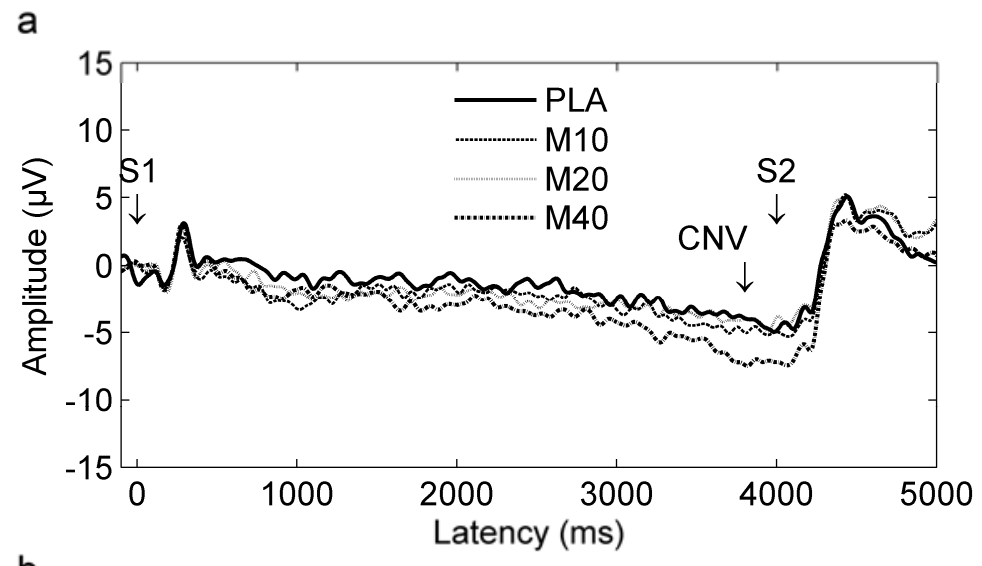

b

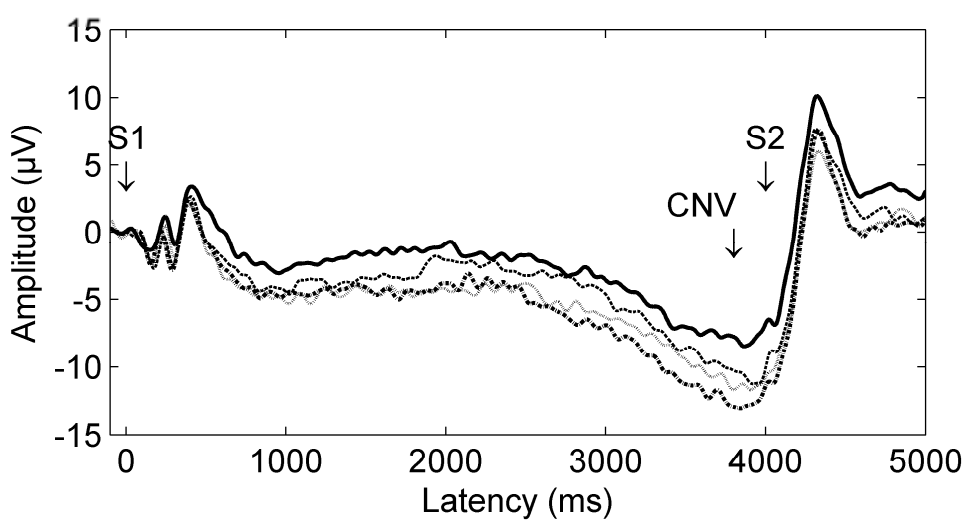

Figure 1. Grand average ERPs showing a clear CNV wave during the Stoplight (a) and Lines 2 (b) response preparation tasks. The ERPs depicted were recorded at $\mathrm{Cz}$, at $\mathrm{t} 240$ relative to dosing. 


\section{Results}

\section{CNV amplitude}

Averaged CNV waves recorded during performance of response preparation tasks are shown in Figure 1. Mean differences on CNV amplitude relative to morning baseline are depicted in Figure 2 for stoplight and lines 2 at $\mathrm{Cz}$. Mean differences on CNV amplitude relative to morning baseline and results of significant difference testing are presented in Table 1.

a

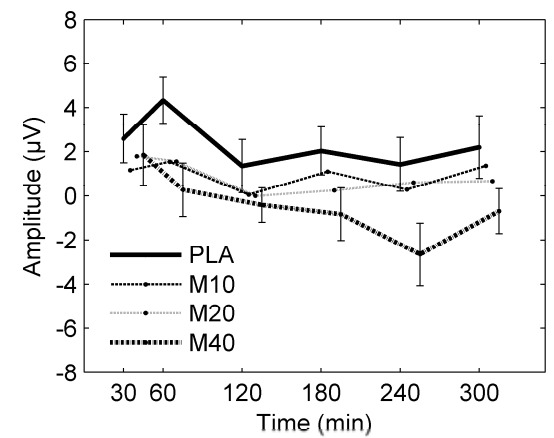

C

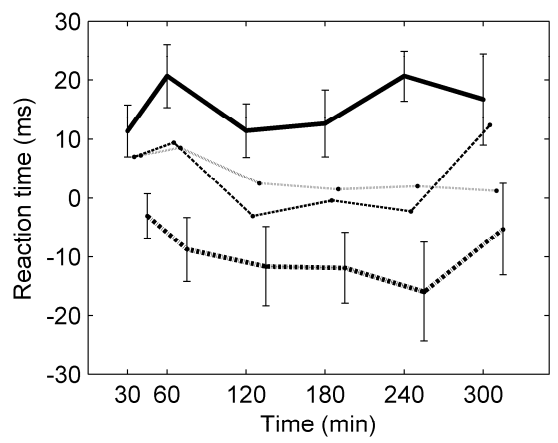

b

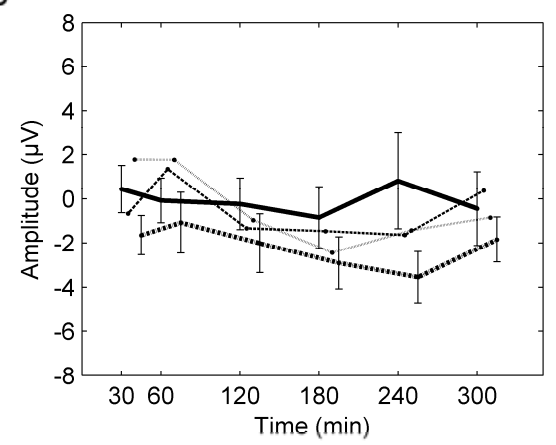

d

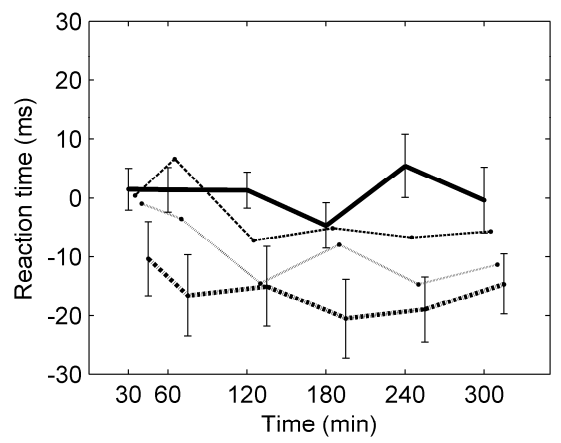

Figure 2. Mean difference CNV amplitudes and reaction times relative to morning baseline at $\mathrm{t} 30$, $60,120,180,240$ and 300 minutes relative to dosing in the Stoplight (amplitudes at Cz, a; reaction times, c) and Lines 2 (amplitudes at $\mathrm{Cz}, \mathbf{b}$; reaction times, d) response preparation tasks. Error bars are only shown for PLA (placebo) and M40 (methylphenidate, $40 \mathrm{mg}$ ). 
Increases in CNV amplitude after methylphenidate measured at $t 60$ and $t 240$ account for, respectively, $27 \%$ and $46 \%$ of the total amount of significant differences observed in all paradigms and at all electrodes. More specifically, the effects at 1 and $4 \mathrm{~h}$ post-dosing were as follows (for a complete overview, see Table 1): Significant increases in CNV amplitude after methylphenidate during performance of the stoplight task were observed at $t 60$ after intake of $40 \mathrm{mg}$ of the drug at $\mathrm{Cz}, \mathrm{C} 3$ and $\mathrm{C} 4$ (Cz: $p<0.013$, C3: $p<0.035, \mathrm{C4}: p<$ $0.049)$. At $t 60$, the amplitude was also larger after $M 10$ at $C z(p<0.045)$. At $t 240, M 40$ lead to a significantly increased amplitude at all three electrodes (Cz: $p<0.032$, C3: $p<0.028$, C4: $p<0.017$ ).

During performance of the lines task version 1, amplitude was significantly larger after $40 \mathrm{mg}$ of methylphenidate compared to placebo at t240 at the $\mathrm{Cz}$ electrode $(p<0.025)$. Analysis of the lines task version 2 showed significantly higher amplitudes after $M 40$ at $t 60$ at the $\mathrm{C} 3$ electrode position $(p<0.035)$ and at $t 240$ at $C 4(p<0.014)$. At $C z$, all doses led to higher CNV amplitude at $t 240$ (M10: $p<0.025, M 20: p<0.014$, M40: $p<0.006$ ).

\section{Reaction time data}

Mean differences on reaction times relative to morning baseline are depicted in Figure 2 for stoplight and lines 2. Mean differences on reaction time relative to morning baseline and results of significant difference testing are presented in Table 2.

Reaction times to the imperative stimulus in the stoplight task were shorter after $40 \mathrm{mg}$ of methylphenidate compared to placebo at all time points. At $t 240$, reaction times were also shorter after 10 and $20 \mathrm{mg}$ of methylphenidate.

On the lines task, version 1 , reaction times were also shorter after $40 \mathrm{mg}$ of methylphenidate at $t 60, t 120, t 240$ and $t 300$. On version 2 of the lines task, reaction times were shorter after $40 \mathrm{mg}$ from $t 60$ to $t 300$ and after all doses at $t 120$. Reaction times were also shorter after the two lower doses at $t 120$ and $t 240$. Finally, M40 decreased reaction times on the lines 3 task on all time points. In addition, reaction times were shorter after $10 \mathrm{mg}$ of methylphenidate at $t 120$ and $t 240$.

\section{Subjective measures}

The Bond \& Lader factor scores indicated that participants felt more alert between 1 and $2 \mathrm{~h}$ after 20 and $40 \mathrm{mg}$ drug intake and more content from 1 to $3 \mathrm{~h}$ after $20 \mathrm{mg}$ of methylphenidate. Furthermore, analysis showed increased scores for calmness after $40 \mathrm{mg}$ of methylphenidate at $t 120$ but decreased scores after $10 \mathrm{mg}$ at $t 180$ and $t 300$. 
Table 1

Mean differences (SEM) on CNV amplitude relative to morning baseline for Stoplight and Lines

\begin{tabular}{|c|c|c|c|c|c|c|c|c|c|c|c|c|}
\hline & \multicolumn{8}{|l|}{$\mathrm{C} 3$} & \multicolumn{4}{|l|}{$\mathrm{Cz}$} \\
\hline & \multicolumn{2}{|c|}{ Stoplight } & \multicolumn{2}{|l|}{ Lines 1} & \multicolumn{2}{|l|}{ Lines 2} & \multicolumn{2}{|l|}{ Lines 3} & \multicolumn{2}{|c|}{ Stoplight } & \multicolumn{2}{|l|}{ Lines 1} \\
\hline & Mean & (SEM) & Mean & (SEM) & Mean & (SEM) & Mean & (SEM) & Mean & (SEM) & Mean & (SEM) \\
\hline \multicolumn{13}{|l|}{ T30 } \\
\hline PLA & 2.21 & $(-0.89)$ & -0.50 & (1.09) & 1.78 & (1.03) & 2.79 & (1.09) & 2.59 & (1.10) & -0.73 & (1.12) \\
\hline M10 & 1.43 & $(0.87)$ & 0.66 & $(0.87)$ & -0.18 & $(0.67)$ & -0.33 & $(0.98)$ & 1.17 & $(0.79)$ & 0.62 & $(0.87)$ \\
\hline M20 & 3.50 & $(1.46)$ & 0.53 & $(0.84)$ & 0.79 & (1.27) & 1.60 & $(0.91)$ & 1.79 & (1.41) & 0.41 & $(0.90)$ \\
\hline M40 & 2.71 & (1.71) & -2.21 & (1.11) & $-1.91^{* *}$ & $(1.03)$ & 1.76 & $(0.96)$ & 1.85 & (1.38) & -1.79 & $(0.94)$ \\
\hline \multicolumn{13}{|l|}{ T60 } \\
\hline PLA & 3.22 & (1.03) & 0.65 & $(0.92)$ & 1.49 & $(0.97)$ & 3.03 & (1.19) & 4.32 & (1.04) & -0.22 & (1.21) \\
\hline M10 & 2.00 & $(1.02)$ & 1.29 & $(1.05)$ & 1.11 & $(1.00)$ & 0.27 & $(0.84)$ & $1.55^{*}$ & $(0.96)$ & 1.24 & (1.11) \\
\hline M20 & 1.81 & $(0.77)$ & -0.02 & (1.08) & 0.22 & $(0.95)$ & $0.35^{\wedge}$ & $(0.79)$ & $1.55^{\wedge}$ & (1.17) & 0.28 & (1.06) \\
\hline M40 & $0.27^{*}$ & $(1.06)$ & -0.50 & $(0.94)$ & $-1.80^{*}$ & $(1.45)$ & 0.52 & $(0.62)$ & $0.27^{*}$ & $(1.22)$ & 0.15 & $(0.93)$ \\
\hline \multicolumn{13}{|l|}{ T120 } \\
\hline PLA & 0.42 & (1.07) & -0.01 & $(1.21)$ & 0.00 & $(1.03)$ & 2.89 & $(1.54)$ & 1.35 & $(1.20)$ & -0.22 & (1.16) \\
\hline M10 & 0.05 & $(0.82)$ & 0.27 & (1.17) & $-1.87^{\wedge}$ & $(0.84)$ & $-1.48^{\wedge}$ & $(0.92)$ & 0.05 & $(0.68)$ & -0.27 & (1.11) \\
\hline M20 & 1.62 & (1.15) & -1.62 & $(1.03)$ & -1.57 & $(0.98)$ & 0.11 & $(1.20)$ & -0.01 & (1.21) & -2.33 & (1.34) \\
\hline M40 & -0.02 & $(0.78)$ & -2.42 & $(0.88)$ & -1.72 & $(1.18)$ & $-0.55^{\wedge}$ & $(0.86)$ & -0.42 & $(0.78)$ & -1.42 & (1.01) \\
\hline \multicolumn{13}{|l|}{ T180 } \\
\hline PLA & 2.37 & $(0.92)$ & -0.32 & $(1.14)$ & -0.07 & $(1.02)$ & 0.20 & $(0.87)$ & 2.03 & (1.09) & -0.60 & (1.05) \\
\hline M10 & 1.28 & $(0.79)$ & -0.20 & $(1.35)$ & -1.08 & $(0.91)$ & -2.73 & $(0.96)$ & 1.10 & $(0.71)$ & 0.06 & (1.02) \\
\hline M20 & $0.74^{\wedge}$ & $(0.89)$ & -1.52 & $(1.08)$ & $-1.61^{\wedge}$ & (1.14) & 0.04 & (1.09) & 0.24 & $(0.98)$ & -2.27 & (1.48) \\
\hline M40 & 0.12 & (1.15) & -2.14 & (1.44) & $-2.89^{*}$ & (1.09) & -1.84 & $(0.77)$ & -0.84 & (1.19) & $-2.96^{\wedge}$ & (1.52) \\
\hline \multicolumn{13}{|l|}{ T240 } \\
\hline PLA & 1.35 & $(1.06)$ & 0.45 & $(0.82)$ & 0.84 & $(1.72)$ & 1.89 & $(1.24)$ & 1.42 & $(1.22)$ & -0.13 & $(0.94)$ \\
\hline M10 & 0.89 & (1.01) & -1.03 & $(0.99)$ & $-1.34^{\wedge}$ & $(0.94)$ & $-2.12^{\wedge}$ & (1.15) & 0.28 & $(0.97)$ & -1.06 & (1.12) \\
\hline M20 & 1.61 & $(1.05)$ & -1.17 & $(0.87)$ & $-1.09^{\wedge}$ & (1.07) & $-1.17^{\wedge}$ & $(0.91)$ & 0.61 & (1.01) & -1.42 & (1.19) \\
\hline M40 & $-2.35^{*}$ & $(1.22)$ & $-2.05^{\wedge}$ & (1.27) & $-1.84^{\wedge}$ & $(1.03)$ & -0.31 & $(0.78)$ & $-2.65^{*}$ & $(1.46)$ & $-3.14^{*}$ & (1.45) \\
\hline \multicolumn{13}{|l|}{ T300 } \\
\hline PLA & 1.21 & (1.15) & 0.50 & $(0.97)$ & 0.12 & $(1.45)$ & 1.60 & (1.18) & 2.20 & (1.41) & -1.26 & $(0.89)$ \\
\hline M10 & 0.75 & (1.01) & -0.34 & (1.15) & -0.22 & (1.07) & -1.12 & $(1.01)$ & 1.37 & $(0.99)$ & -0.42 & (1.34) \\
\hline M20 & 1.82 & $(1.04)$ & -0.71 & $(0.96)$ & -0.60 & $(0.79)$ & -0.62 & $(1.07)$ & 0.67 & (1.16) & -1.24 & (1.14) \\
\hline M40 & -0.63 & $(0.99)$ & -0.44 & $(0.98)$ & -0.03 & $(0.77)$ & -0.93 & $(1.10)$ & -0.70 & $(1.05)$ & -2.66 & $(1.04)$ \\
\hline
\end{tabular}

${ }^{\wedge} p<.1 ;{ }^{*} p<0.05{ }^{* *} ; p<.01 ;{ }^{* * *} p<.001$ (signigicant effects and trends). 
tasks at $\mathrm{C} 3, \mathrm{Cz}$, and $\mathrm{C} 4$ at all time points

\begin{tabular}{|c|c|c|c|c|c|c|c|c|c|c|c|}
\hline \multirow[b]{2}{*}{ Lines 2} & \multirow[b]{3}{*}{ (SEM) } & \multirow{2}{*}{\multicolumn{2}{|c|}{ Lines 3}} & \multicolumn{8}{|l|}{$\mathrm{C} 4$} \\
\hline & & & & Stoplig & & Lines 1 & & Lines 2 & & Lines & \\
\hline Mean & & Mean & (SEM) & Mean & (SEM) & Mean & (SEM) & Mean & (SEM) & Mean & (SEM) \\
\hline 0.44 & (1.07) & 2.74 & (1.13) & 2.61 & $(0.86)$ & -0.32 & $(0.94)$ & 0.99 & (1.12) & 2.69 & (1.10) \\
\hline-0.67 & $(0.83)$ & $-0.90^{\wedge}$ & $(1.03)$ & 1.01 & $(0.59)$ & 0.29 & $(0.76)$ & -0.14 & $(0.82)$ & -0.65 & $(0.82)$ \\
\hline 1.78 & (1.57) & 0.97 & (1.08) & 2.32 & $(0.96)$ & -0.12 & $(0.78)$ & 2.01 & (1.26) & 1.96 & $(0.87)$ \\
\hline$-1.65^{\wedge}$ & $(0.89)$ & 1.16 & (1.34) & 2.10 & (1.20) & -1.61 & $(0.87)$ & $-1.46^{*}$ & $(0.98)$ & 0.79 & (1.29) \\
\hline-0.08 & (1.01) & 1.85 & $(1.40)$ & 3.63 & $(0.78)$ & -0.11 & $(0.98)$ & 0.65 & (1.01) & 1.42 & (1.17) \\
\hline 1.35 & (1.25) & 0.66 & (1.14) & 1.49 & $(0.91)$ & 1.67 & $(0.96)$ & 1.52 & $(0.97)$ & 0.77 & (1.22) \\
\hline 1.77 & $(1.34)$ & 0.68 & $(1.14)$ & $1.01^{\wedge}$ & $(0.97)$ & 0.93 & (1.04) & 1.39 & $(0.98)$ & 0.59 & $(0.90)$ \\
\hline-1.08 & $(1.37)$ & 1.00 & $(0.88)$ & $0.52^{*}$ & $(1.14)$ & 0.18 & $(0.93)$ & $-1.40^{\wedge}$ & (1.21) & 0.23 & $(0.75)$ \\
\hline-0.23 & (1.17) & 2.21 & (1.51) & 1.28 & $(0.98)$ & -0.33 & $(1.22)$ & 0.62 & $(0.98)$ & 1.77 & (1.32) \\
\hline-1.34 & $(0.860$ & -1.40 & $(0.99)$ & 0.26 & $(0.64)$ & 0.41 & $(0.94)$ & $-1.30^{\wedge}$ & $(0.79)$ & -1.01 & $(0.75)$ \\
\hline-0.97 & $(0.82)$ & -0.16 & (1.28) & 0.67 & $(1.06)$ & -1.38 & (1.29) & -0.01 & $(0.98)$ & 0.68 & (1.10) \\
\hline$-2.02^{\wedge}$ & (1.33) & $-0.95^{\wedge}$ & (1.13) & -0.50 & $(0.65)$ & -1.15 & $(0.83)$ & $-1.64^{\wedge}$ & (1.16) & 0.09 & $(0.90)$ \\
\hline-0.85 & $(1.40)$ & -0.27 & $(0.99)$ & 2.57 & $(0.89)$ & -0.19 & $(0.90)$ & 0.05 & (1.15) & 0.32 & $(0.88)$ \\
\hline-1.46 & (1.11) & -2.30 & (1.03) & 1.19 & $(0.62)$ & 0.36 & (1.14) & -1.46 & (1.13) & -1.59 & $(0.76)$ \\
\hline$-2.44^{\wedge}$ & $(1.34)$ & -1.01 & (1.49) & 0.67 & $(0.81)$ & -1.00 & (1.14) & -1.61 & $(1.22)$ & 0.21 & (1.25) \\
\hline$-2.91^{\wedge}$ & (1.17) & -1.97 & $(0.83)$ & 0.64 & $(1.25)$ & $-1.94^{*}$ & (1.29) & -1.53 & $(1.20)$ & -1.55 & $(0.60)$ \\
\hline 0.81 & $(2.17)$ & 0.66 & (1.41) & 1.96 & $(0.85)$ & 0.22 & $(0.75)$ & 0.65 & (1.61) & 1.00 & (1.35) \\
\hline$-1.64^{*}$ & (1.13) & -2.43 & (1.17) & 0.29 & $(0.73)$ & 0.03 & $(1.02)$ & -0.55 & (1.04) & -1.28 & $(0.99)$ \\
\hline$-1.43^{*}$ & (1.38) & $-2.14^{\wedge}$ & (1.18) & 0.47 & (1.04) & -0.66 & (1.10) & $-0.40^{\wedge}$ & (1.17) & -0.08 & (1.07) \\
\hline$-3.55^{* *}$ & (1.19) & -1.62 & $(0.89)$ & $-2.15^{*}$ & $(1.72)$ & $-1.82^{\wedge}$ & (1.39) & $-2.58^{*}$ & $(1.00)$ & -0.83 & (0.99) \\
\hline-0.45 & (1.67) & -0.01 & (1.11) & 2.34 & $(0.98)$ & 0.01 & $(0.97)$ & 1.00 & (1.64) & 0.58 & $(0.87)$ \\
\hline 0.37 & (1.21) & -1.47 & (1.14) & 2.22 & $(0.99)$ & -0.04 & (1.01) & 0.64 & $(0.80)$ & -0.78 & $(0.96)$ \\
\hline-0.85 & $(0.96)$ & -1.91 & (1.35) & 1.27 & $(0.99)$ & -0.07 & $(0.95)$ & 0.02 & $(1.02)$ & -0.47 & (1.13) \\
\hline-1.85 & $(1.04)$ & $-2.65^{\wedge}$ & $(0.82)$ & $-0.84^{*}$ & $(0.89)$ & $-1.24^{\wedge}$ & $(0.73)$ & -0.73 & $(0.90)$ & -0.82 & $(0.70)$ \\
\hline
\end{tabular}


Analysis of POMS factor scores indicated that after methylphenidate, participants experienced improved mood (all doses) and less anger (20 mg) compared to placebo between 1 and $3 \mathrm{~h}$ after drug intake. Factor scores indicated less fatigue at $t 60, t 120$ and $t 240$ after 20 and $40 \mathrm{mg}$ of methylphenidate, more vigour from 1 to $4 \mathrm{~h}$ after methylphenidate intake (all doses) and less tension after $40 \mathrm{mg}$ of methylphenidate at $t 30$ and $t 300$.

Table 2

Mean differences (SEM) on reaction times relative to morning baseline for Stoplight and Lines task

\begin{tabular}{|c|c|c|c|c|c|c|c|c|c|}
\hline & & Stoplight & & Lines 1 & & Lines 2 & & Lines 3 & \\
\hline & & Mean & (SEM) & Mean & (SEM) & Mean & (SEM) & Mean & (SEM) \\
\hline \multirow[t]{4}{*}{ T30 } & PLA & 11.4 & (4.4) & 5.4 & (4.4) & 1.5 & (3.5) & -0.1 & (4.3) \\
\hline & M10 & 7.0 & (6.3) & 4.0 & (3.3) & 0.4 & $(4.6)$ & -5.5 & (2.6) \\
\hline & M20 & 7.3 & $(4.9)$ & 6.2 & $(4.0)$ & -1.0 & (3.1) & 1.2 & $(4.1)$ \\
\hline & M40 & $-3.1^{*}$ & (3.8) & -3.6 & (5.6) & -10.3 & (6.3) & $-12.6^{*}$ & (5.4) \\
\hline \multirow[t]{4}{*}{ T60 } & PLA & 20.7 & (5.4) & 5.9 & (4.6) & 1.4 & (3.8) & 2.4 & $(4.2)$ \\
\hline & M10 & 9.4 & (7.1) & 5.7 & (3.7) & 6.6 & $(6.0)$ & 0.7 & (5.4) \\
\hline & M20 & 8.5 & $(6.0)$ & 1.7 & (3.7) & -3.5 & $(4.8)$ & -6.3 & (4.4) \\
\hline & M40 & $-8.7^{* *}$ & (5.4) & $-9.4^{*}$ & (5.9) & $-16.5^{*}$ & $(6.9)$ & $-14.8^{*}$ & (6.5) \\
\hline \multirow[t]{4}{*}{ T120 } & PLA & 11.4 & (4.5) & 6.0 & (4.6) & 1.3 & (3.0) & -3.7 & $(4.5)$ \\
\hline & M10 & -3.1 & (6.4) & -2.0 & (2.7) & $-7.2^{*}$ & (3.5) & $-11.7^{* *}$ & (2.7) \\
\hline & M20 & 2.5 & $(4.4)$ & -4.4 & (3.1) & $-14.5^{*}$ & (3.4) & -11.9 & (3.9) \\
\hline & M40 & $-11.6^{* *}$ & $(6.8)$ & $-9.5^{*}$ & (5.5) & $-15.0^{*}$ & $(6.8)$ & $-20.2^{* *}$ & $(6.0)$ \\
\hline \multirow[t]{4}{*}{ T180 } & PLA & 12.6 & (5.7) & 0.6 & $(4.6)$ & -4.7 & (3.9) & -6.3 & $(5.2)$ \\
\hline & M10 & -0.4 & (9.3) & 1.2 & (2.9) & -5.1 & $(4.2)$ & -8.7 & (3.0) \\
\hline & M20 & 1.5 & (5.9) & -1.5 & (2.7) & -8.0 & $(3.2)$ & -10.2 & $(5.2)$ \\
\hline & M40 & $-11.9^{*}$ & (6.1) & $-11.2^{\wedge}$ & (5.8) & $-20.5^{*}$ & (6.7) & $-22.0^{* *}$ & $(6.2)$ \\
\hline \multirow[t]{4}{*}{ T240 } & PLA & 20.7 & (4.3) & 3.5 & (3.8) & 5.4 & (5.4) & -4.3 & (5.2) \\
\hline & M10 & $-2.3^{*}$ & (9.1) & -3.0 & (2.7) & $-6.7^{* *}$ & (4.1) & $-11.6^{*}$ & (2.2) \\
\hline & M20 & $2.0^{*}$ & (3.9) & -1.6 & (4.9) & $-14.6^{* *}$ & (3.8) & -10.8 & (5.1) \\
\hline & M40 & $-15.9^{* * *}$ & $(8.6)$ & $-10.2^{*}$ & (5.7) & $-18.9^{* * *}$ & (5.7) & $-19.1^{* *}$ & $(5.8)$ \\
\hline \multirow[t]{4}{*}{ T300 } & PLA & 16.7 & (7.8) & 4.4 & (4.5) & -0.4 & (5.6) & -5.8 & (5.4) \\
\hline & M10 & 12.4 & (8.1) & 2.2 & (3.4) & -5.6 & (4.7) & -8.5 & (2.6) \\
\hline & M20 & $1.2^{\wedge}$ & $(5.0)$ & -2.9 & (3.1) & -11.3 & (3.5) & -14.2 & (4.9) \\
\hline & M40 & $-5.3^{*}$ & (8.0) & $-7.0^{* *}$ & (4.7) & $-14.6^{*}$ & (5.2) & $-16.8^{*}$ & $(5.8)$ \\
\hline
\end{tabular}

${ }^{\wedge} \mathrm{p}<.1 ;{ }^{*} \mathrm{p}<0.05 ;{ }^{* *} \mathrm{p}<.01 ;{ }^{* * *} \mathrm{p}<.001$ (significant effects and trends). 
Table 3

Means (SEM) of cortisol and prolactin levels in blood, absolute baseline values and mean differences (SEM) relative to morning baseline for heart rate, systolic and diastolic blood pressure (BP) at all time points

\begin{tabular}{|c|c|c|c|c|c|c|c|c|c|c|c|}
\hline & & \multicolumn{2}{|c|}{ Cortisol } & \multicolumn{2}{|c|}{ Prolactin } & \multicolumn{2}{|c|}{ Heart Rate } & \multicolumn{2}{|c|}{ BP systolic } & \multicolumn{2}{|c|}{ BP diastolic } \\
\hline & & Mean & (SEM) & Mean & (SEM) & Mean & (SEM) & Mean & (SEM) & Mean & (SEM) \\
\hline \multirow[t]{4}{*}{$\mathrm{BL}$} & PLA & 491 & (27) & 11.4 & $(0.9)$ & 66.3 & $(2.2)$ & 133.9 & (3.3) & 73.8 & $(3.8)$ \\
\hline & M10 & 504 & $(28)$ & 11.6 & $(1.2)$ & 66.7 & (2.7) & 130.6 & $(2.5)$ & 71.8 & $(2.8)$ \\
\hline & M20 & 576 & $(27)$ & 13.6 & (1.3) & 67.7 & $(2.3)$ & 133.1 & (3.0) & 70.7 & $(1.7)$ \\
\hline & M40 & 512 & $(43)$ & 11.8 & $(1.2)$ & 66.3 & (2.5) & 133.2 & (2.7) & 72.7 & (1.7) \\
\hline \multirow[t]{4}{*}{ T30 } & PLA & & & & & -2.1 & (1.4) & -0.2 & $(1.8)$ & -0.5 & $(1.6)$ \\
\hline & M10 & & & & & -3.1 & (1.4) & -1.0 & (2.1) & -0.8 & $(3.0)$ \\
\hline & M20 & & & & & -2.5 & (1.1) & -1.8 & (2.0) & 0.8 & (1.3) \\
\hline & M40 & & & & & -2.9 & $(0.9)$ & -1.8 & (2.0) & 1.1 & (1.7) \\
\hline \multirow[t]{4}{*}{ T60 } & PLA & 325 & (21) & 9.0 & $(0.6)$ & -6.8 & $(1.2)$ & -6.8 & (2.7) & -2.7 & $(3.2)$ \\
\hline & M10 & $405^{*}$ & (35) & $7.6^{* *}$ & $(1.0)$ & -3.6 & $(2.0)$ & -1.5 & $(1.6)$ & 2.2 & (3.9) \\
\hline & M20 & $454^{* *}$ & (31) & $7.0^{* * *}$ & $(0.6)$ & $-0.5^{* *}$ & (1.4) & $0.9^{* *}$ & (2.3) & $6.4^{* *}$ & $(1.5)$ \\
\hline & M40 & $510^{* * *}$ & $(32)$ & $6.4^{* * *}$ & $(0.9)$ & $2.9^{* * *}$ & (2.1) & $2.9^{* * *}$ & (2.4) & $5.7^{* *}$ & (2.3) \\
\hline \multirow[t]{4}{*}{ T120 } & PLA & 300 & (18) & 8.5 & $(0.7)$ & -9.6 & (1.1) & -5.5 & $(1.8)$ & -0.6 & (2.9) \\
\hline & M10 & 332 & (30) & $6.4^{* * *}$ & $(0.9)$ & $-5.8^{*}$ & (1.4) & $2.3^{*}$ & (2.4) & 2.2 & (3.3) \\
\hline & M20 & $409^{* *}$ & (31) & $5.8^{* * *}$ & $(0.7)$ & $-2.4^{* * *}$ & $(1.4)$ & $0.8^{*}$ & (2.6) & $6.6^{*}$ & (1.7) \\
\hline & M40 & $481^{* * *}$ & (35) & $4.4^{* * *}$ & $(0.4)$ & $0.9^{* * *}$ & $(2.2)$ & $3.7^{* * *}$ & (2.3) & $5.6^{* *}$ & (2.2) \\
\hline \multirow[t]{4}{*}{ T180 } & PLA & 313 & (21) & 7.9 & $(0.7)$ & -9.8 & (1.1) & -4.6 & (2.3) & -1.7 & (3.8) \\
\hline & M10 & 347 & (25) & $6.4^{* * *}$ & $(0.8)$ & $-5.5^{*}$ & (1.6) & $2.0^{*}$ & (1.5) & 0.4 & (3.3) \\
\hline & M20 & $411^{* *}$ & (28) & $5.8^{* * *}$ & $(0.7)$ & $-2.6^{* * *}$ & (1.6) & $0.7^{*}$ & (2.1) & $6.1^{* *}$ & (1.9) \\
\hline & M40 & $411^{* * *}$ & (30) & $4.9^{* * *}$ & $(0.7)$ & $1.3^{* * *}$ & (1.8) & -0.2 & (2.5) & $3.3^{*}$ & (1.6) \\
\hline \multirow[t]{4}{*}{ T240 } & PLA & 400 & (31) & 10.1 & (1.1) & 0.2 & (1.6) & -0.4 & (2.5) & -5.0 & (3.0) \\
\hline & M10 & 419 & (24) & 9.1 & $(0.8)$ & 1.3 & (1.6) & $5.0^{*}$ & (2.1) & -0.1 & (3.9) \\
\hline & $M 20$ & 423 & (28) & $8.0^{* *}$ & $(0.8)$ & $4.9^{* *}$ & $(2.0)$ & $5.3^{*}$ & $(2.0)$ & $2.9^{*}$ & (1.5) \\
\hline & M40 & 457 & (24) & $6.8^{* *}$ & $(0.8)$ & $7.9^{* * *}$ & (1.8) & 2.9 & (1.9) & $2.5^{* *}$ & (1.6) \\
\hline \multirow[t]{4}{*}{ T300 } & PLA & 281 & (16) & 9.8 & $(0.8)$ & 1.0 & (1.6) & -3.0 & (2.4) & -3.1 & (3.0) \\
\hline & M10 & 312 & (21) & 9.3 & $(1.0)$ & 0.5 & (1.6) & $3.7^{*}$ & $(1.8)$ & 0.3 & (3.2) \\
\hline & M20 & $354^{* *}$ & (18) & 9.6 & $(1.0)$ & $4.4^{*}$ & (1.3) & 1.2 & (2.3) & 2.2 & (1.3) \\
\hline & M40 & $369^{* * *}$ & (19) & 8.0 & $(0.7)$ & $6.6^{* *}$ & $(1.6)$ & -1.9 & (2.2) & 0.4 & (1.9) \\
\hline
\end{tabular}

${ }^{\wedge} \mathrm{p}<.1 ;{ }^{*} \mathrm{p}<0.05 ;{ }^{* *} \mathrm{p}<.01 ;{ }^{* * *} \mathrm{p}<.001$ (significant effects and trends). 


\section{Blood plasma values}

Mean levels of cortisol and prolactin in blood and results of significant difference testing are presented in Table 3. Methylphenidate significantly increased cortisol levels. At $t 60$, cortisol levels were significantly higher after all doses of methylphenidate when compared to placebo $\left(F_{3,41.43}=9.207, p<\right.$ $0.001)$. At $t 120\left(F_{3,40.71}=11.964, p<0.001\right), t 180\left(F_{3,41.86}=8.238, p<0.001\right)$ and $t 300\left(F_{3,39.02}=8.663, p<0.001\right)$ cortisol levels were significantly higher after $M 20$ and $M 40$ but not $M 10$. At $t 240$, there was no effect of drug on cortisol levels $\left(F_{3,39.24}=0.647, p>0.05\right)$.

Prolactin levels were lower after all doses of methylphenidate in comparison to placebo from $t 60$ to $t 180$ ( $t 60: F_{3,36.32}=13.247, p<0.001 ; t 120$ : $\left.F_{3,38.18}=32.293, p<0.001, t 180: F_{3,39.97}=29.558, p<0.001\right)$ and after $M 20$ and $M 40$ at $t 240\left(F_{3,37.11}=4.702, p<0.01\right)$ At $t 300$, the effect was no longer significant $\left(F_{3,37.52}=0.945, p>0.05\right)$.

Maximum methylphenidate concentration in blood differed significantly between treatment conditions $\left(C_{\max }: M 10\right.$ mean $(\mathrm{SEM})=4.9(0.4)$; M20 mean $(\mathrm{SEM})=9.3(0.7)$; M40 mean $(\mathrm{SEM})=17.2(1.2), F_{2,30}=140.130, p<$ $0.001)$. $T_{\max }$ was equal for all treatments (mean $(\mathrm{SEM})=2.9(0.2), F_{2,30}=$ $0.134, p>0.05)$.

\section{Physiological measures}

Absolute baseline values of physiological measures and mean differences relative to morning baseline and results of significant difference testing are presented in Table 3. Methylphenidate $(20$ and $40 \mathrm{mg}$ ) significantly increased heart rate compared to placebo from 1 up to $5 \mathrm{~h}$ after drug administration $\left(t 60: F_{3,43.88}=10.194, p<0.001 ; t 120: F_{3,44.04}=14.395, p<0.001 ; t 180: F_{3,42.78}\right.$ $=13.325, p<0.001 ; t 240: F_{3,38.97}=9.195, p<0.001 ; t 300: F_{3,41.84}=7.271, p<$ 0.001 ). At $t 120$ and $t 180$, all doses significantly increased heart rate. Systolic blood pressure was significantly higher after $M 20$ and M40 compared to placebo at $t 60\left(F_{3,40.13}=5.433, p<0.01\right)$ and after all doses at $t 120\left(F_{3,40.46}=\right.$ $5.178, p<0.01$ ). At $t 180, t 240$ and $t 300$, there was no main effect of drug on systolic blood pressure, but systolic blood pressure was higher compared to placebo after $M 10$ ( $t 180: p<0.05 ; t 240: p<0.05 ; t 300: p<0.05$ ) and $M 20$ $(t 180: p<0.05$; t240: $p<0.05)$ as was revealed by the planned contrasts. Diastolic blood pressure significantly increased $1 \mathrm{~h}$ after intake of 20 and $40 \mathrm{mg}$ of methylphenidate compared to placebo (t60: $F_{3,42.39}=5.279, p<$ $0.01)$. This effect remained significant until $4 \mathrm{~h}$ after drug intake $\left(t 120: F_{3}\right.$, $43.39=4.808, p<0.01 ; t 180: F_{3,43.73}=4.468, p<0.01 ; t 240: F_{3,42.04}=3.796, p<$ $0.05)$. 


\section{Discussion}

In the current study, the effects of different doses of methylphenidate on the late CNV wave were assessed in order to explore the utility of the CNV amplitude as a marker of dopamine activity. In line with the expectations, methylphenidate dose dependently increased CNV amplitude and decreased reaction time in both response preparation paradigms. Furthermore, subjective measures indicated that participants experienced improved mood, felt more alert and vigorous and less angry and tired after methylphenidate. Physiological measures including blood pressure and heart rate increased, confirming previous findings (Volkow, et al., 1996). These results indicate that dopamine activity increases response readiness as measured by the CNV paradigm.

CNV has been previously reported to be sensitive to dopaminergic modulation (Amabile, et al., 1986; Kopell, et al., 1974; Oishi, et al., 1995; Tecce, 1991; Tecce \& Cole, 1974; Tecce, et al., 1975). However, little was known about dose-related effects. In the present study, the effects on reaction time and CNV amplitude could be detected after a dose of methylphenidate as low as $10 \mathrm{mg}$, demonstrating the sensitivity of the CNV paradigm to changes in dopamine availability. In support of a dose-response relationship, the effects were most prominently and consistently observed after the highest dose of methylphenidate. Importantly, the current effects are measured on the late CNV wave. Previous studies, using shorter intervals between S1 and S2 (Luthringer, et al., 1999), may possibly have measured a composite of the early and late wave. An internal validation of the paradigms used in this study is provided by correlation analysis, which revealed consistent correlations between the difference scores for response time and CNV amplitude at $\mathrm{Cz}$ in the $40 \mathrm{mg}$ of methylphenidate condition in all paradigms at all time points, ranging between 0.46 and 0.96 with average of 0.78. This correlation also emerged for the lower doses but a little less consistent, which is again indicative of a dose-response relationship. The lower doses may generate more variance which may less often lead to significant correlations.

Because methylphenidate inhibits re-uptake of dopamine, but also of noradrenaline (Arnsten \& Dudley, 2005), it cannot be stated with absolute certainty whether the current findings should be ascribed solely to increased dopamine levels. Dopamine levels in the brain cannot be measured directly, but changes in prolactin level in blood are a good surrogate marker since the prolactin level is known to rise as the dopamine level decreases and vice versa (Ben-Jonathan, 1985). The decrease in prolactin levels observed here suggests that methylphenidate did successfully increase central dopamine levels. 
Methylphenidate not only enhanced CNV amplitude but also increased response speed. As Fan et al., (2007) suggested, it seems that anticipation of the response, as reflected by CNV amplitude, improves response preparation and execution. Between S1 and S2, two waves can be observed, the early and the late CNV wave (Klorman \& Bentsen, 1975). In this study, the late CNV wave was investigated. The late wave is thought to be a mixture of a readiness potential and stimulus preceding negativity (Brunia \& van Boxtel, 2001). Improved performance may be the result of better motor preparation, increased anticipatory attention or a combination of both.

Based on the literature, it was expected that methylphenidate levels in the blood would peak $2 \mathrm{~h}$ after drug intake (Volkow, Fowler, Wang, Ding, \& Gatley, 2002). In the current study, $T_{\max }$ was observed somewhat later, almost $3 \mathrm{~h}$ after intake. Although methylphenidate reaches a plateau between 2 and $3 \mathrm{~h}$, a delay in reaching $T_{\max }$ may possibly be related to the composition of the breakfast (Patrick, et al., 2007). Behavioural effects already appeared at 30 min after drug intake and were still present after $5 \mathrm{~h}$. The effects on the EEG measures were more dispersed in time. Moreover, the effects clustered mainly around two time points, at approximately 1 and $4 \mathrm{~h}$ after drug intake. In other words, the effects are observed relatively shortly after drug intake and $1 \mathrm{~h}$ after $T_{\max }$.

The current method does not allow source localization. However, previous research suggests that the CNV is generated in the basal ganglia (Fan, et al., 2007; Y. Nagai, et al., 2004). Taking into account the neuroanatomic projections of the dopaminergic pathways, the effect observed $1 \mathrm{~h}$ after drug intake may be mediated by a fast response of the nigrostriatal pathway, being responsible for an early attention enhancing effect. This idea is supported by our observation that participants felt more alert this time point. The late effect, on the other hand, is more likely to be mediated by the mesocortical pathway, which may be responsible for the top-down control of anticipatory processes affecting response readiness (Brunia \& van Boxtel, 2001; Fan, et al., 2007). Alternatively, the schedule at testing days may have played a role in the timing of the effects. Whereas participants were probably rather energetic at $t 60$ and $t 240$, before which they just had a lunch break, they may have been less focused at $t 120$ and $t 180$. However, this only partly fits with the subjective effects reported by the participants.

The pattern of effects was quite similar for behavioural and EEG data. Both measures were modulated the most in the stoplight and lines 2 version of the task. There were only subtle differences between these tasks with respect to the methylphenidate effect on both response time and amplitude. However, since the lines 2 version is a little more attractive to participants, this task may be preferred for future use. 
Generalization of the results may be limited by the fact that only male participants were included. Furthermore, taking into account the number of tasks administered, the testing days may have been rather demanding for the participants. This may have caused detrimental effects on performance or decrease of motivation. A limitation of the Bond and Lader visual analogue scale is that the calmness scale is based on very few (two) items. Therefore, interpretation of this scale warrants caution.

In conclusion, the current findings indicate that methylphenidate dose dependently increases response readiness. In sum, the CNV appears to be a good candidate biomarker for assessing changes in the dopaminergic function by treatments that either directly or indirectly target the dopamine system. Future research should aim to dissect the pharmacological selectivity of the CNV. 
Chapter 7. Effects of methylphenidate and levodopa on the Contingent Negative Variation

Submitted for publication

\section{Abstract}

The Contingent Negative Variation (CNV) is a slow negative shift in the EEG and is thought to be correlate of attention and psychomotor function. Previous CNV research suggests that there is potential for the CNV to be used as a biomarker for dopamine activity. However, selectivity of the CNV for dopamine activity remains to be determined. To investigate the specific dopaminergic influence on the CNV amplitude the effects of $40 \mathrm{mg}$ methylphenidate (MPH) on CNV were compared to those of $100 \mathrm{mg}$ of the dopamine precursor levodopa (LEV) in a placebo-controlled crossover study testing 30 healthy volunteers. In addition, participants completed two visual analogue scales and heart rate and blood pressure were monitored. MPH and LEV had opposite effects on CNV amplitude and reaction time. MPH increased CNV amplitude and decreased reaction time in comparison with LEV. It is suggested that combined dopamine- and noradrenaline reuptake inhibition may be essential to enhance response readiness and that MPH effects may be more pronounced if conditions are more demanding. 


\section{Introduction}

As an electrophysiological correlate of attention and psychomotor function, the Contingent Negative Variation (CNV) is studied extensively in clinical as well as psychopharmacological experiments. The CNV is a slow negative shift in the electroencephalogram (EEG) that appears between a warning and imperative stimulus in a reaction time task. CNV amplitude is reduced in Parkinson Disease patients (lkeda, et al., 1997; Pulvermuller, et al., 1996; Verleger, et al., 1999; Wascher, et al., 1997) and may also be affected in patients with schizophrenia, ADHD, depression and Alzheimer's Disease (Golob \& Starr, 2000; Kratz, et al., 2009; Timsit-Berthier, 1991; van Deursen, et al., 2009; Verleger, et al., 1999). Therapeutic response to drug treatment in these patient groups may be reflected by normalization of CNV amplitude.

The underlying mechanism for the observed alterations in the CNV in neuropsychiatric patients is likely to be controlled by influences from GABAergic, cholinergic, serotonergic, dopaminergic, and noradrenergic neurotransmitter systems (Timsit-Berthier, 1991). The exact nature of some of these influences remains to be fully understood but previous research has shown that benzodiazepines, including diazepam, nitrazepam and clonazepam reduce CNV amplitude (Ashton, Millman, Telford, \& Thompson, 1974; Ashton, et al., 1976; Papart, et al., 1997; Rockstroh, et al., 1991). Increases in acetylcholine levels induced by nicotine intake show inconsistent, possibly biphasic effects on the CNV (Ashton, et al., 1980; Ashton, et al., 1974; Knott \& Venables, 1980; Rockstroh, et al., 1991). Conflicting results are reported for serotonin (Hansenne, et al., 2000; Zank, Strehl, Larbig, \& Kotchoubey, 2008). It has been proposed that serotonin may affect CNV through its effects on the catecholaminergic system (Mulder, et al., 2002).

The catecholamines noradrenaline and dopamine are thought to play an important role in the generation of the CNV (Timsit-Berthier, et al., 1986) and underlie alterations in the CNV amplitude in migraine patients (Mulder, et al., 2002). Furthermore, methylphenidate, a catecholamine reuptake inhibitor, showed dose-related effects on response readiness, reflected by increased CNV amplitude and faster responses to the imperative stimulus (Linssen, et al., 2011). Noradrenaline involvement in CNV is also suggested by the effect of clonidine, a noradrenergic drug that acts through the alphaadrenergic receptors and has a biphasic effect on CNV amplitude, showing an initial increase and later decrease of CNV amplitude (Ashton \& Rawlins, 1978). However, propranolol, a noradrenergic drug that acts through the betaadrenergic receptors did not affect CNV amplitude (Ashton, et al., 1976).

Evidence of dopaminergic effects on CNV includes the observation that reduced CNV amplitude can be restored by levodopa treatment in 
Parkinson's patients (Amabile, et al., 1986; Oishi, et al., 1995). In healthy individuals, manipulation of dopamine levels may also induce changes in CNV amplitude (Tecce, 1991). CNV amplitude has been shown to reduce with dopamine antagonists, such as chlorpromazine (Tecce 1975). In sum, previous research on pharmacological influences on the CNV shows that several neurotransmitters can mediate changes in CNV amplitude, but a major role is played by the catecholamines.

Previous CNV research suggests that there is potential for the CNV to be used as a biomarker (Linssen, et al., 2011; Tecce \& Cole, 1974). Besides normalization of CNV amplitude in neuropsychiatric patients in response to treatment, CNV may also predict therapeutic response to pharmacological treatment of migraine (Schoenen, Maertens de Noordhout, Timsit-Berthier, \& Timsit, 1986). Specifically, CNV is proposed as a marker for dopaminergic neurotransmission (Luthringer, et al., 1999). Support for the CNV as a suitable biomarker is provided by a recent study demonstrating the sensitivity of the CNV amplitude; it was affected even by a low drug dose and its response was dose dependent (Linssen, et al., 2011). These results were obtained with methylphenidate, which inhibits the reuptake of both dopamine and noradrenaline transporters. Therefore, it is not clear to what extent dopamine and noradrenaline individually contribute to methylphenidate's effect on response readiness. It is suggested that methylphenidate's potency may be higher for the noradrenaline- than the dopamine transporter (Kuczenski \& Segal, 1997), but as dopamine is also transported by the noradrenaline transporter, it is not clear if noradrenaline or dopamine is more affected by methylphenidate (Hannestad, et al., 2010; Pacholczyk, et al., 1991). Hence, this study does not give conclusive support for the CNV as a biomarker that is selective for dopamine activity.

Considering the number of neuropsychiatric diseases that involve dopamine abnormalities including Parkinson's Disease, Schizophrenia and $A D H D$ and the amount of literature that supports a role for dopamine in modulation of the CNV amplitude, the validation of CNV as a marker for dopamine activity is both valuable and promising. Therefore, the current study investigates the specific dopaminergic influence on the CNV amplitude by comparing the effects of methylphenidate on CNV to those of the DA precursor levodopa. This enables us to compare the effects of a selectively dopaminergically acting drug to that of a mixed dopamine-noradrenaline acting drug.

On separate occasions, participants were treated with $40 \mathrm{mg}$ of methylphenidate, $125 \mathrm{mg}$ of levodopa/carbidopa and placebo. The latter drug consists of $100 \mathrm{mg}$ of levodopa and $25 \mathrm{mg}$ of carbidopa. Levodopa is transformed into dopamine both in the brain as well as in the body. Carbidopa 
does not cross the blood brain barrier and peripherally inhibits the enzyme dopa decarboxylase which converts levodopa to dopamine (Julien, 2005). Consequently, more levodopa can be transported to the brain and less side effects will develop.

In addition to CNV amplitude, task performance and subjective drug effects were also measured. Based on research cited above, noradrenergic and dopaminergic influences might both be expected to affect CNV amplitude and this may be reflected by differential effects of methylphenidate versus levodopa. However, as the evidence for dopamine involvement in the CNV is more substantial, it was hypothesised that CNV amplitude would be similarly positively affected by levodopa and methylphenidate.

\section{Method}

\section{Participants}

Twenty healthy male volunteers and 10 healthy female volunteers (mean age $=20.7, \mathrm{SD}=2.3$, range=18-28) were selected and paid to participate. Participants were recruited by means of local advertisements. Prescreening occurred using a medical history questionnaire and was followed by medical examination.

The main inclusion criteria were: between 18 and 45 years of age, body mass index between 18 and $30 \mathrm{~kg} / \mathrm{m}^{2}$ and right-handedness. The main exclusion criteria were history or presence of mental or physical disorders, consumption of more than 21 alcohol units per week or more than five caffeine-containing drinks per day, pregnancy or lactation, use of medication other than oral contraceptives, use of recreational drugs from 2 weeks before until the end of the experiment. All subjects gave written informed consent. The study was carried out in accordance with the Declaration of Helsinki and approved by the medical ethical committee of Maastricht University.

\section{Design}

The study was conducted according to a double-blind, placebo-controlled, three-way crossover design. Between the testing days, a period of at least 48 $\mathrm{h}$ elapsed, but generally, testing days were scheduled approximately 1 week apart. Each participant received one of three single treatments including placebo (PLA), $40 \mathrm{mg}$ of methylphenidate (MPH) and $125 \mathrm{mg}$ of levodopa/carbidopa (LEV) on each testing day. $T_{\max }$ is approximately 2 hours for MPH and 1 hour for LEV(Chana, Fierro, Reyes-Parada, \& Saez-Briones, 2003; Leonard, et al., 2004). LEV was given in combination with $10 \mathrm{mg}$ of domperidone (a peripherally acting dopamine antagonist) to prevent nausea. Six different treatment sequences were used, with each sequence occurring 
4, 5 or 6 times. MPH was administered upon arrival at 8.30 or 9.00 (T0). Due to the different half life, LEV was administered an hour later (T60).

\section{Dependent measures}

\section{EEG measurement}

During two response preparation tasks, EEG was measured using 11 electrodes placed on the scalp, according to the 10-20 system at the Fz, F3, F4, Cz, C3, C4, C3', C4', Pz, P3 and P4 electrode positions. The ground electrode was placed on the AFz location, on the forehead. Two electrodes placed on the mastoids served as reference channels. An electro-oculogram (EOG) was measured bipolarly vertically above and below the right eye and horizontally next to the right and the left eye. Data were sampled at $250 \mathrm{~Hz}$ and filtered online between 0.05 and $100 \mathrm{~Hz}$. Offline, data were first screened for artefacts and a low pass filter of $10 \mathrm{~Hz}$ was applied on all data. Next, epochs were extracted from the continuous EEG. Each epoch lasted $5100 \mathrm{~ms}$, starting $100 \mathrm{~ms}$ before the warning stimulus ( $\mathrm{S} 1$ ) and ending $1000 \mathrm{~ms}$ after the imperative stimulus (S2) of the reaction time tasks. A baseline correction was applied to the whole epoch, using the $100 \mathrm{~ms}$ interval preceding $\mathrm{S} 1$ as reference. Averaging the sequential epochs yielded a S1 stimulus locked event-related potential (ERP). CNV amplitude was calculated by averaging the amplitude over the last $100 \mathrm{~ms}$ before S2. Since the CNV amplitude was expected to be maximal at the central electrodes (Brunia, 1988; Klorman \& Bentsen, 1975; van Boxtel \& Brunia, 1994), C3, C3', Cz, C4, C4, P3, Pz and P4 were of main interest and are reported in the Results section. In the results section, the lateral electrodes will be referred to as $C_{\text {same, }} C_{\text {opposite, }} C^{\prime}$ same, $\mathrm{C}^{\prime}$ opposite, $\mathrm{P}_{\text {same }}$ and $\mathrm{P}_{\text {opposite, }}$, depending on their position relative to the response finger (e.g. $C_{\text {same }}$ includes results of $\mathrm{C} 3$ for right finger responses and $\mathrm{C} 4$ for left finger responses).

\section{Response preparation tasks \\ CNV traffic light task}

In the CNV traffic light task, participants watched the computer screen and were presented with a filled red circle (S1) on a black background. After $4 \mathrm{~s}$, the red circle turned green (S2). Subjects were instructed to respond to the green circle by pressing a button on a table mounted response box as fast as they could. The test consisted of 32 trials. Reaction time to the onset of S2 was measured in each trial and averaged over the test.

CNV Lines task

In the CNV lines task, S1 was the appearance of two red line drawings at the bottom left- and right-hand corner. The lines were oriented at a $90^{\circ}$ angle to each other and after appearing both moved towards the centre of the screen. 
After $4 \mathrm{~s}$, the lines crossed and turned green (S2). At this moment, participants pressed a response button as fast as possible. As movement of the lines allows better estimation of the occurrence of the imperative stimulus, participants' responses are faster compared to the traffic light task. To introduce different levels of difficulty, in half of the trials, a horizontal yellow bar partially blocked the view of the lines as they moved towards the centre of the screen, but the lines were always visible at the moment of crossing. Participants were cued to either respond with the left or right index finger by the presentation of an arrow pointing in either direction throughout the trial in order to balance lateralization effects. Participants responded with the left and right index finger equally often; numbers were balanced across bar and no bar trials and the order was randomized. The test consisted of 100 trials. Reaction time was measured and averaged per trial type (bar vs no bar).

\section{Subjective measures}

Participants completed two rating scales of subjective effects, the Profile of Mood States (POMS; (MCNair, et al., 1992)) and the Bond and Lader (B\&L) visual analogue scales (Bond \& Lader, 1974). These rating scales consisted of 16 (B\&L) or 32 (POMS) bipolar items. Each item consisted of two words describing mood states. Participants had to score their preference for either of the items in accordance with their mood by marking the line closer to one of the two words. Dependent measures were factor scores on the dimensions Alertness (B\&L), Depression, Anger, Fatigue, Vigour and Tension (POMS). Both rating scales were administered at four time points (T0, T50, T130 and T180) on each testing day.

\section{Physiological measures}

Physiological measures included systolic and diastolic blood pressure and heart rate and were taken at four time points (T0, T50, T130 and T180) on each testing day.

\section{Procedure}

The current study was integrated in a larger study on the effects of methylphenidate and levodopa on cognition and psychomotor function. A training session in which the tasks were practiced took place within 2 weeks before the first testing day. Participants abstained from alcohol during the last $24 \mathrm{~h}$ prior to each testing day. On testing days, participants were either collected at their home or came by public transport. They arrived at the lab in fasted condition at either 8.30 am or 9:00 am. Due to the different half lives of the medication, drug administration occurred at different time points, 
MPH at $T=0$ and LEV at $T=60$. To ensure double blinding, participants received identically appearing capsules at all time points on each testing day. Participants were given a standardized meal for breakfast. Then, the electrode cap was placed and participants could relax until actual testing started at $T=120$. First, a baseline EEG measurement was taken, followed by cognitive testing in the following order: Visual verbal learning test, Sternberg memory scanning task, delayed recall and recognition test of the visual verbal learning test, paired associates learning and spatial working memory task. In addition, two blood samples were taken at $T=120$ and $T=180$. Subjective and physiological measures were taken at four time points (T0, T50, T130 and T180). At the end of the testing day, participants were returned to their home by car.

\section{Data analysis}

Data were analyzed using a repeated measures analysis of variance with the within-subjects factor treatment, which had three levels: PLA, MPH and LEV. Since the effects of MPH and LEV were anticipated to go into the same direction, main effects of the drugs were not evaluated. Instead, a priori planned comparisons between each dose relative to placebo were tested for significance at $p<0.05$, using LSD correction.

\section{Results}

\section{CNV amplitude}

Averaged CNV waves recorded during performance of response preparation tasks are shown in Figure 1. Mean values of the CNV amplitude and p-values for the planned comparisons are presented in Table 1. Neither MPH nor LEV affected the CNV amplitude during performance of the traffic light task (Table 1). During performance of the CNV Lines task, CNV amplitude increased significantly after MPH at the lateral P-electrode opposite $\left(\mathrm{P}_{\mathrm{opp}}\right)$ to the response finger ( $\mathrm{P} 3$ in case of right finger response and $\mathrm{P} 4$ in case of left finger response) on trials with a yellow bar partially blocking the view on the lines. In trials where there was no bar, MPH significantly increased CNV amplitude at $\mathrm{Cz}$ and Pz electrodes. Pairwise comparisons for LEV vs. PLA were not significant, but CNV amplitude was significantly smaller after LEV compared to $\mathrm{MPH}$ for bar-trials at $\mathrm{Cz}, \mathrm{C}_{\text {opposite, }} \mathrm{C}^{\prime}$ same, $\mathrm{C}_{\text {opposite, }} \mathrm{P}_{\text {same, }} \mathrm{Pz}$ and $\mathrm{P}_{\text {opposite }}$ electrodes and for trials without a bar at $\mathrm{Cz}, \mathrm{C}_{\text {opposite, }} \mathrm{C}^{\prime}$ same, $\mathrm{C}^{\prime}$ opposite and $\mathrm{P}_{\text {opposite }}$ electrodes. 
a

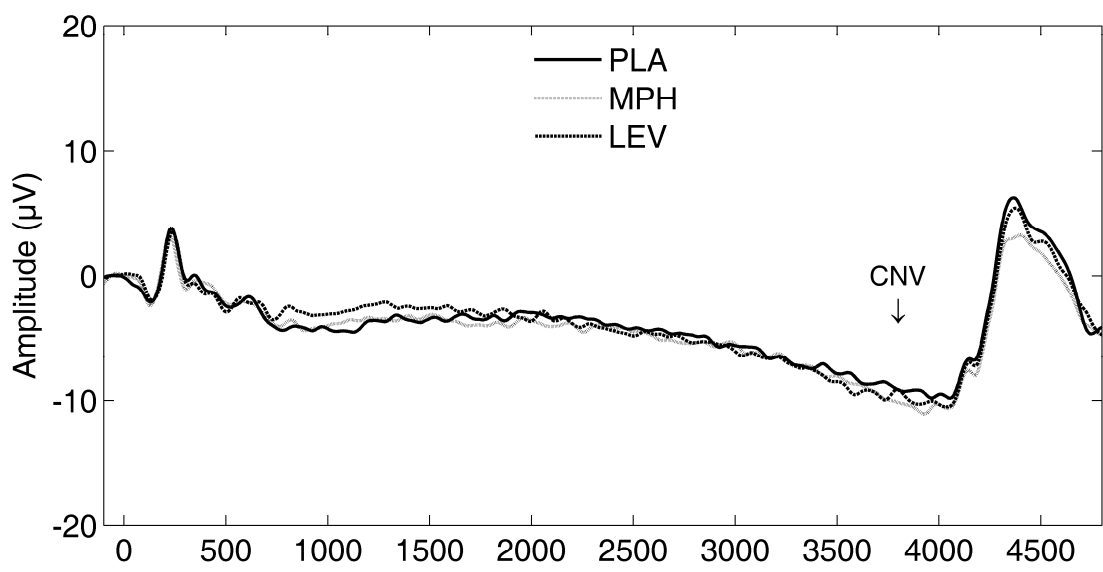

b

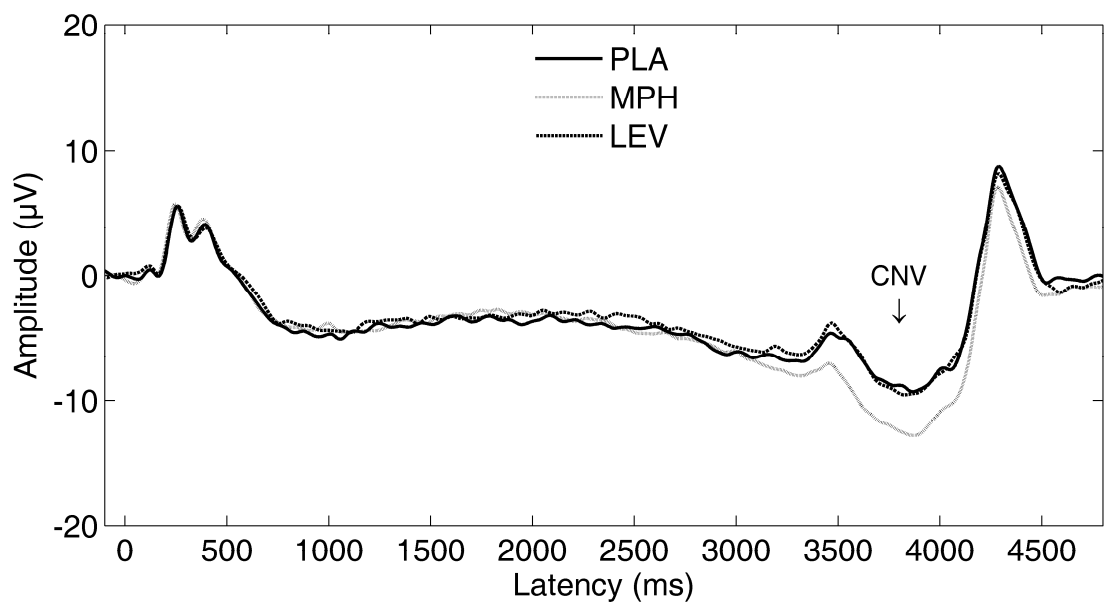

Figure 1. Grand average ERPs showing a CNV wave during the CNV traffic light task (a) and CNV Lines (No bar) task (b). The ERPs depicted were recorded at $\mathrm{Cz}$.

\section{Reaction time data}

Mean reaction times for CNV traffic light and Lines tasks are presented in Table 2. Response time was faster after MPH compared to PLA on the trials with a bar in the CNV Lines tasks. Furthermore, contrasts show (Table 2) that for the CNV traffic light task and both CNV Lines tasks, response times were significantly faster after MPH compared to LEV 
Table 1

Mean values (SEM) of CNV amplitudes after PLA, MPH and LEV and p-values of contrasts PLA vs. $M P H, P L A$ vs. LEV and MPH vs. LEV for the three CNV tasks (TL=CNV Traffic Light).

\begin{tabular}{|c|c|c|c|c|c|c|c|c|c|}
\hline & \multicolumn{6}{|c|}{ Mean amplitude (SEM) per treatment } & \multicolumn{3}{|c|}{ Contrasts (p) } \\
\hline & PLA & & $\mathrm{MPH}$ & & LEV & & PLA vs. & PLA vs. & $\mathrm{MPH}$ vs. \\
\hline TL & & & & & & & $\mathrm{MPH}$ & LEV & LEV \\
\hline Copposite & -7.50 & $(0.85)$ & -8.12 & $(0.90)$ & -7.22 & $(0.94)$ & 0.526 & 0.784 & 0.267 \\
\hline C'opposite & -9.09 & $(0.99)$ & -10.18 & $(1.21)$ & -8.74 & $(1.30)$ & 0.392 & 0.774 & 0.193 \\
\hline $\mathrm{Cz}$ & -9.85 & $(1.05)$ & -11.07 & $(1.30)$ & -10.33 & (1.17) & 0.368 & 0.678 & 0.493 \\
\hline C'same & -8.50 & $(0.99)$ & -8.85 & $(1.23)$ & -9.12 & $(1.26)$ & 0.770 & 0.512 & 0.815 \\
\hline Csame & -6.40 & $(0.71)$ & -7.52 & $(1.11)$ & -6.86 & $(0.79)$ & 0.261 & 0.516 & 0.484 \\
\hline Popposite & -5.64 & $(0.97)$ & -6.65 & (1.07) & -6.24 & $(1.28)$ & 0.354 & 0.645 & 0.716 \\
\hline $\mathrm{Pz}$ & -7.05 & (1.05) & -7.79 & $(1.11)$ & -7.57 & $(1.10)$ & 0.572 & 0.666 & 0.820 \\
\hline Psame & -5.58 & $(0.91)$ & -6.66 & $(0.97)$ & -5.60 & $(0.89)$ & 0.292 & 0.985 & 0.157 \\
\hline \multicolumn{10}{|l|}{ Lines bar } \\
\hline Copposite & -8.09 & $(0.88)$ & -8.87 & $(0.82)$ & -7.59 & $(0.65)$ & 0.220 & 0.466 & $0.033^{*}$ \\
\hline C'opposite & -9.55 & $(1.00)$ & -10.66 & $(0.99)$ & -8.94 & $(0.79)$ & 0.163 & 0.568 & $0.023^{*}$ \\
\hline $\mathrm{Cz}$ & -7.41 & $(0.91)$ & -8.03 & $(0.68)$ & -6.31 & $(0.66)$ & 0.489 & 0.243 & $0.014^{*}$ \\
\hline C'same & -8.89 & $(0.94)$ & -9.60 & $(0.91)$ & -7.98 & $(0.82)$ & 0.430 & 0.284 & $0.012^{*}$ \\
\hline Csame & -6.97 & $(0.78)$ & -7.35 & $(0.78)$ & -6.35 & $(0.66)$ & 0.587 & 0.368 & 0.101 \\
\hline Popposite & -6.75 & $(1.06)$ & -8.21 & $(0.94)$ & -5.98 & $(0.64)$ & $0.026^{*}$ & 0.309 & $0.001^{*}$ \\
\hline $\mathrm{Pz}$ & -8.26 & $(1.03)$ & -8.96 & $(0.94)$ & -7.16 & $(0.86)$ & 0.381 & 0.232 & $0.016^{*}$ \\
\hline Psame & -6.41 & $(0.94)$ & -7.07 & $(0.90)$ & -5.53 & $(0.63)$ & 0.256 & 0.199 & $0.016^{*}$ \\
\hline \multicolumn{10}{|c|}{ Lines no bar } \\
\hline Copposite & -8.38 & (1.01) & -9.69 & $(0.90)$ & -8.06 & $(1.02)$ & 0.105 & 0.697 & $0.010^{*}$ \\
\hline C'opposite & -10.19 & $(1.10)$ & -11.12 & $(1.03)$ & -8.94 & (1.13) & 0.321 & 0.163 & $0.012^{*}$ \\
\hline $\mathrm{Cz}$ & -10.38 & $(1.16)$ & -12.40 & $(1.07)$ & -9.98 & (1.27) & 0.027 & 0.703 & $0.009^{*}$ \\
\hline C'same & -8.57 & $(1.02)$ & -10.19 & $(0.90)$ & -8.37 & $(1.13)$ & 0.071 & 0.812 & $0.045^{*}$ \\
\hline Csame & -6.94 & $(0.90)$ & -7.48 & $(0.75)$ & -6.40 & $(1.12)$ & 0.487 & 0.518 & 0.178 \\
\hline Popposite & -5.85 & $(1.04)$ & -7.04 & $(0.92)$ & -5.51 & (1.17) & 0.070 & 0.680 & $0.049^{*}$ \\
\hline $\mathrm{Pz}$ & -5.25 & $(1.17)$ & -6.92 & $(0.97)$ & -5.14 & $(1.22)$ & 0.040 & 0.913 & 0.060 \\
\hline Psame & -5.22 & $(0.92)$ & -6.12 & $(0.84)$ & -4.55 & (1.11) & 0.199 & 0.447 & 0.059 \\
\hline
\end{tabular}

${ }^{*} \mathrm{p}<.05$

\section{Subjective measures}

Analysis of POMS factor scores indicated that after methylphenidate, participants experienced less fatigue at 1 and 3 hours after drug intake $(p=.014$ and $p=.019$ respectively). Methylphenidate also caused an increase in reported tension at 1 hour and 2 hours after administration $(p=.040$ and $\mathrm{p}=.015$ respectively). Factor scores indicated less vigour at 1 and 3 hours after 
methylphenidate intake $(p=.003$ and $p=.005$ respectively). The Bond \& Lader factor scores indicated that participants felt more alert 3 hours after MPH intake $(\mathrm{p}=.037)$.

Table 2

Mean values (SEM) of response times after PLA, MPH and LEV and p-values of contrasts PLA vs. $M P H, P L A$ vs. LEV and MPH vs. LEV

\begin{tabular}{lllllllllll}
\hline & \multicolumn{4}{l}{ Mean response times (SEM) } & \multicolumn{4}{c}{ Contrasts (p) } \\
\hline & PLA & \multicolumn{3}{c}{ MPH } & LEV & & PLA vs. & PLA vs. & MPH vs. \\
& & & & & & & MPH & LEV & LEV \\
TL & 223.2 & $(6.4)$ & 215.9 & $(5.4)$ & 226.4 & $(5.4)$ & 0.095 & 0.525 & $0.008^{*}$ \\
Lines bar & 61.9 & $(3.5)$ & 53.3 & $(3.8)$ & 63.4 & $(3.9)$ & $0.010^{*}$ & 0.603 & $0.006^{*}$ \\
Lines no bar & 47.5 & $(3.4)$ & 44.4 & $(3.3)$ & 49.5 & $(3.4)$ & 0.205 & 0.346 & $0.043^{*}$ \\
\hline
\end{tabular}

${ }^{*} \mathrm{p}<.05$.

\section{Physiological measures}

MPH significantly increased heart rate compared to PLA at all time points (T50: $\mathrm{p}<.001 ; \mathrm{T} 130: \mathrm{p}<.001 ; \mathrm{T} 180: \mathrm{p}<.001$ ). Furthermore, compared to PLA, MPH also significantly increased systolic at all time points (T50: $p<.001 ;$ T130: $\mathrm{p}<.001 ; \mathrm{T} 180: \mathrm{p}<.001)$ and diastolic blood pressure (T50: $\mathrm{p}<.001 ; \mathrm{T} 130$ : $\mathrm{p}=.002 ; \mathrm{T} 180: \mathrm{p}=.002$ ). LEV did not affect heart rate and systolic blood pressure at $T 130$, but significantly decreased diastolic blood pressure relative to PLA $(p=.047)$. In both PLA and LEV, heart rate decreased from T0 to T180, but this decrease was significantly smaller after LEV $(p=.035)$. At T180, there were no effects of LEV on blood pressure.

\section{Discussion}

In the current study, the effects of $\mathrm{MPH}$-induced dopamine and noradrenaline reuptake inhibition on the CNV were compared to those of a selectively dopaminergic drug, LEV. Contrary to our expectations, the effects of MPH on CNV amplitude and reaction time were opposite to those of LEV. MPH increased CNV amplitude in the Lines task relative to LEV and, to a lesser extent also relative to PLA. Similarly, MPH speeded responses compared to LEV in all tasks, and in the CNV Lines-bar task participants were also faster after MPH compared to PLA. Subjective measures indicated that participants experienced less fatigue, more tension, and less vigour after MPH and felt more alert after $\mathrm{MPH}$. These results do not support the hypothesis that the effect of $\mathrm{MPH}$ on CNV amplitude and response time is specifically related to its dopamine enhancing effect. 
Previous research has shown that catecholamines modulate CNV amplitude (Timsit-Berthier, et al., 1986). Most studies have focused on dopaminergic influences on the CNV and it has been suggested that the CNV may be a good candidate biomarker of dopamine activity (Linssen, et al., 2011; Luthringer, et al., 1999; Tecce, 1991), also since a recent study showed dose-dependent effects of MPH on CNV amplitude and response time. The current study aimed to determine the extent to which dopamine, rather than noradrenaline, enhancement accounts for the MPH effects on the CNV by comparing $\mathrm{MPH}$ to a selective dopaminergic drug. The present findings do not support the hypothesis that MPH induced CNV enhancement is primarily mediated by dopamine. However, it is important to consider the differences between the mechanisms of the drugs used. MPH tonically increases extracellular dopamine in the striatum and prefrontal cortex (Leonard, et al., 2004), while LEV increases presynaptic dopamine availability, affecting both tonic as well as phasic dopaminergic neurotransmission (Breitenstein, Korsukewitz, et al., 2006).

Intuitively, one might expect that the impact of dopamine enhancement by LEV may thus be relatively larger than that of $\mathrm{MPH}$. Dopaminergic effects on performance are often described by an inverted $U$ curve in which intermediate dopamine levels are associated with an optimal state and a higher dose may cause adverse effects, i.e. in this study, decreased readiness to respond, reflected by smaller CNV amplitude and slower responses (Levy, 2009; Mehta, et al., 2004). However, to achieve such high levels of dopamine activity, a relatively high dose of a dopaminergic agent is needed. Increased tonic dopamine activity reduces the phasic release of dopamine by binding to postsynaptic- and autoreceptors (Breitenstein, Floel, et al., 2006; Seeman \& Madras, 2002). Low doses of a dopamine agonist or LEV thus result in less dopamine activity at the post-synapse (Seeman \& Madras, 2002). Higher doses overcome this effect and cause widespread stimulation (Seeman \& Madras, 2002). Although LEV has the potential to enhance tonic as well as phasic dopamine activity, it is possible that the combined actions resulted in a state with no net change in dopamine levels, as the LEV dose used in the current study was relatively low $(100 \mathrm{mg})$. This dose was used to prevent sedation. Sedation is seen with doses of $200 \mathrm{mg}$ (Andreu, et al., 1999; Micallef-Roll, Rihet, Hasbroucq, Possamai, \& Blin, 2001) while behavioural effects have been observed with $100 \mathrm{mg}$ (Breitenstein, Floel, et al., 2006; Knecht, et al., 2004).

Hence, it is possible that the LEV dose was too low to enhance CNV amplitude or speed responses. However, that does not explain why the LEV effects go in the opposite direction compared to MPH. Although the LEV effects did not significantly differ from PLA, the averages show a consistent 
pattern in which MPH leads to the highest CNV amplitude and the fastest responses; LEV induces the lowest CNV amplitude and slowest responses; and intermediate values are observed with PLA.

It is more likely that the MPH effect on CNV amplitude and response time is not primarily dopamine mediated. This does not necessarily mean that the MPH effect is purely noradrenergic. The combined dopamine and noradrenaline reuptake inhibition may be essential to enhance response readiness. The MPH effect might be explained by the additive effects of enhanced motor function mediated by dopamine enhancement (Nieoullon, 2002) and noradrenergically mediated effects on attention, alertness (Breitenstein, Floel, et al., 2006; Coull, Frith, Dolan, Frackowiak, \& Grasby, 1997). Indeed, subjects reported to experience less fatigue after MPH and felt more alert after $\mathrm{MPH}$. Although these subjective effects cannot completely account for the current results, they may affect participants' motivation to perform the task (Gevins, Smith, \& McEvoy, 2002). In studies testing high performing healthy volunteers, subjective feelings and motivational effects may be a source of variability in an otherwise homogeneous group.

Ideally this study would have disentangled dopaminergic and noradrenergic effects by including a purely noradrenergic substance. Based on previous studies, commonly used noradrenergic drugs were regarded unsuitable for this aim because of non-specificity, lack of safety or adverse side effects that would interfere with task performance. For example, as dopamine is also transported by the noradrenaline transporter, a noradrenaline reuptake inhibitor would not reveal purely noradrenergic effects (Hannestad, et al., 2010; Pacholczyk, et al., 1991).

Regardless of the dopaminergic contribution to the MPH effect on CNV, LEV was expected to enhance CNV amplitude, based on previous findings in Parkinson's Disease patients. These patients show reduced CNV amplitude, which can be restored by LEV treatment (Amabile, et al., 1986; Oishi, et al., 1995). The absence of a CNV increasing effect in healthy volunteers suggests that pure dopamine enhancement only enhances CNV amplitude in individuals with suboptimal dopamine levels in the brain. Performance of CNV tasks by healthy volunteers may not benefit from additional dopaminergic activity alone because their dopamine levels are already close to optimal.

In our previous study, MPH enhanced CNV amplitude and decreased reaction time compared to PLA (Linssen, et al., 2011). In the current study, only few significant differences were observed between MPH and PLA. There may be several reasons for the apparent dissimilarity between these results. First of all, the timing of CNV task performance relative to dosing was different from the previous study. In the previous study CNV tasks were administered at T30, T60, T120, T180, T240 and T300 while in the current 
study CNV measurement occurred at T150. As an example, the previous study observed CNV enhancing effects in the CNV traffic light task of MPH at T60 and T240 (i.e. not at T120 or T180). The current study only administered this task at T150, a time point at which no effect was observed in the previous study. The current result is thus in line with the previous study. The MPH effect on CNV is time dependent and does not occur at all time points after dosing.

Secondly, the study procedure of the current study was much less demanding than that of the previous study. In the previous study, participants were subjected to seven test cycles spanning approximately 8 hours with few short breaks in between. It is possible that the stimulating effects of MPH were more pronounced in the previous study due to fatigue induced by continuous computer task performance, leading to a relative reduction in performance in the PLA condition. This reasoning is in line with a previous study that showed performance enhancing effects of MPH after sleep deprivation but not after normal sleep (Bishop, et al., 1997).

In conclusion, the current findings indicate that MPH and LEV, in the doses given in this study, have opposite effects on CNV amplitude and reaction time. $\mathrm{MPH}$ increased $\mathrm{CNV}$ amplitude and decreased reaction time in comparison with LEV. It is suggested that combined dopamine- and noradrenaline reuptake inhibition may be essential to enhance response readiness and that $\mathrm{MPH}$ effects may be more pronounced if task conditions are more demanding. 
GENERAL DISCUSSION 
The present dissertation aimed to investigate the role of dopamine in cognitive function (part 1) and study the CNV as a potential dopaminergic biomarker (part 2). In the first part, dopaminergic involvement in cognitive function in healthy volunteers was studied by means of a literature review and three experimental studies. The second part started with the evaluation of a new CNV paradigm under normal conditions which was subsequently studied under the influence of dopamine enhancing drug treatment. In this general discussion, the results are linked to provide a coherent view of the role of dopamine in cognition and to evaluate the CNV as a dopaminergic biomarker.

\section{The role of dopamine in cognition in the healthy population}

To gain more knowledge about the role of dopamine in cognitive function, this dissertation describes the effects of pharmacological manipulations that alter dopamine activity in the healthy brain. Modulation of dopamine activity may involve either an increase or a decrease in dopamine activity. Dopamine activity can be enhanced by drugs such as methylphenidate and levodopa. According to a current belief among the general population, methylphenidate may enhance cognitive performance in healthy individuals. In order to test this idea, a literature review was performed on the effects of methylphenidate on cognitive function in healthy volunteers. In two experimental studies the methylphenidate effect on several cognitive tasks was further tested and compared to the effect of levodopa. A third experiment tested the cognitive effects of reduced dopamine activity induced by tyrosine/phenylalanine depletion.

\section{Verbal learning and memory}

Results from the literature review in chapter 1 showed that methylphenidate improved verbal learning and memory in healthy volunteers in more than one third of all previous studies. Enhanced performance was mainly observed on word list learning tasks. Strikingly, low and intermediate doses of methylphenidate were more effective than higher doses. In a minority of cases high doses even led to decreased memory performance on short-term verbal memory tasks.

The experimental studies described in part 1, studied the effects of dopaminergic manipulations on word list learning. As previous studies that failed to detect performance modulating effects of methylphenidate used relatively short lists of 15 words, a ceiling effect may have occurred. Therefore, the studies in this dissertation presented participants with lists of 30 words, decreasing the likelihood that participants reached ceiling performance under placebo conditions. This left room for methylphenidate to 
improve memory performance, which was expected based on previous studies reviewed in chapter 1.

The studies in chapter 2 and 3 demonstrated that this expectation was only confirmed under specific circumstances. Chapter 2 showed that when participants were subjected to demanding study procedures, methylphenidate enhanced memory consolidation within 30 minutes after word learning in a dose-related fashion. If participants, on the other hand, relaxed before task performance, performance was not affected by methylphenidate at the 30 minute delay (chapter 3). However, the latter study showed that methylphenidate did improve memory consolidation over a 24 hour delay. Together, the results of the first two studies may thus suggest that memory enhancing effects of methylphenidate are potentially more pronounced when the circumstances are more challenging. While the first study was very demanding for participants because they continuously performed computer tasks over a period of 8 hours, the study procedures of the second study were much shorter, but required to remember words over a 24 hour delay (chapter $3)$. It is possible that under these circumstances, healthy volunteers may benefit more from the stimulant effects of methylphenidate than under normal circumstances.

The study in chapter 3 aimed to determine the dopaminergic contribution to the effects of methylphenidate, which inhibits the reuptake of both noradrenaline and dopamine. Since levodopa, a pure dopaminergic drug, failed to affect memory performance, this study did not appear to provide evidence in favor of the idea that the methylphenidate effect on cognitive function was primarily mediated by dopamine. Based on these behavioral results, one might be tempted to conclude that the memory enhancing effect of methylphenidate was mediated by noradrenaline or that the combination of noradrenaline and dopamine reuptake inhibition led to better memory. However, the ERP results suggested that levodopa slowed down memory processing and led to increased effort. As the levodopa dose was relatively low it is not unlikely that levodopa failed to enhance dopamine activity. In fact, levodopa may even have reduced phasic dopamine activity relative to the placebo condition (Breitenstein, Korsukewitz, et al., 2006; Paulus \& Trenkwalder, 2006; Seeman \& Madras, 2002), an idea supported by the ERP results. Hence, the results in this dissertation do not dismiss the idea of a dopaminergic contribution to methylphenidate's effects on memory. In this context it is important to note that although levodopa and methylphenidate both affect dopamine activity, they differ in mechanisms by which they do so. This issue is discussed in more depth in the 'Methodological considerations'.

The effect of a reduction of dopamine activity on word learning and recall as achieved by depletion of the dopamine precursors tyrosine and 
phenylalanine was discussed in chapter 4. It was hypothesized that dopamine depletion would disturb verbal learning. However performance on the word learning test was not affected by this treatment. Results from the simultaneous EEG measurement, though, suggested that tyrosine/phenylalanine depletion did have adverse effects on memory processing. EEG may be more sensitive than behavioral measures, and hence, small effects may be detected in the EEG, without being expressed in behavior.

While there is some support for the idea that dopamine may be involved in essential memory processing and contributes to optimal memory performance, the limited robustness of memory enhancing effects of methylphenidate, combined with a lack of behavioral effects of levodopa and dopamine depletion suggest that dopamine plays only a restricted role in verbal learning and memory. Furthermore, it is important to note that conclusions from the studies in this dissertation regarding the dopaminergic contribution to cognitive function are limited by the fact that methylphenidate is not a pure dopaminergic drug and that the levodopa manipulation likely did not lead to enhanced dopamine activity.

\section{Working memory}

Previous research has shown that dopamine is necessary for working memory and that both too little as well as too much dopamine can have deleterious effects on working memory performance (Cools \& Robbins, 2004). The effects of several dopamine modulating manipulations were described in part 1 of this dissertation. From the literature review in chapter 1, it was concluded that of all cognitive domains, working memory was most affected by methylphenidate. Nearly $70 \%$ of published results revealed performance enhancing effects of methylphenidate on working memory tasks. Based on the positive association between dopamine and working memory described in the literature, methylphenidate effects on working memory were thought to be primarily mediated by dopamine.

Therefore, when comparing the effects of methylphenidate to those of the pure dopaminergic drug levodopa in the study described in chapter 3 , we expected to observe working memory enhancing effects of both drugs. In contrast with this expectation, methylphenidate led to minor improvement of working memory and levodopa to a minor decrease in working memory performance. These results were unexpected in two respects. First, the drug effects were smaller than expected, as working memory performance only differed between the two drug conditions and not relative to placebo. Second, the drug effects went in opposite directions. As suggested above, levodopa may have failed to enhance dopamine activity, but this does not 
explain how levodopa could lead to a reduction in performance. Neither can it be explained by a noradrenergic component in the methylphenidate effect. The minor decrease in performance once again supported the idea that levodopa decreased the impact of phasic dopamine activity relative to placebo. When we view these results in the light of previous literature on the positive association between dopamine and working memory it is most likely that the levodopa manipulation did not have the intended enhancing effect on dopamine activity.

While working memory enhancement was expected after dopamine enhancing drugs, dopamine depletion was hypothesized to have a negative impact on working memory. This hypothesis was tested in chapter 4. Although tyrosine/phenylalanine depletion failed to alter working memory task performance, results from the simultaneous EEG measurement suggested that tyrosine/phenylalanine depletion had adverse effects on brain processing related to working memory, which remained sub threshold on the behavioral level.

Overall, the results from the research in this thesis suggest that methylphenidate may have a small but positive effect on working memory. We could not prove that the methylphenidate effect on working memory was dopamine dependent, but the suggestion that levodopa reduced phasic dopamine signals, would support a positive association between working memory performance and dopamine activity.

\section{Other cognitive processes}

Dopamine neurotransmitter activity has been associated with various cognitive functions, but most evidence is available for its involvement in motor control and motivated behavior (reward and punishment). In the literature review on methylphenidate effects (chapter 1 ) the full spectrum of cognitive functions was considered. The results showed that, after working memory, speed of processing was the cognitive domain that was most enhanced by methylphenidate. Low doses of methylphenidate were most successful, revealing a pattern consistent with an inverted $\mathrm{U}$ curve describing the relationship between dopamine activity and performance.

Contrary to our expectations, the reviewed literature revealed only a minor influence of methylphenidate on attention and vigilance. Reasoning and problem solving were also hardly affected by methylphenidate, while visual learning and memory showed a complete lack of performance enhancing effects of methylphenidate. The experimental study described in chapter 2 included two attention tasks that were both positively affected by methylphenidate. However, we found no effect on problem solving. 
In sum, the results from the review and the experimental studies demonstrate performance enhancing effects of methylphenidate of varying degrees in several cognitive subdomains. It is important to note that methylphenidate effects on speed of processing may obscure performance enhancing effects on cognitive tasks. In our studies, performance was not impaired by methylphenidate due to a speed-accuracy trade-off. However, in cognitive tasks requiring a motor response, improved performance may partly reflect increased speed of processing.

\section{CNV as a dopaminergic biomarker}

The CNV is a slow negative shift in the EEG that can be observed during response preparation to an anticipated stimulus. A first step in the validation of the CNV as a dopaminergic biomarker was the optimization of the paradigm to induce a CNV wave. While a CNV can be induced by a simple reaction time task called the Traffic light task in which participants press a button when a red dot turns green, the task could be improved to make it more attractive and thereby improve response preparation. The experiments described in chapter 5 evaluated two new tasks that were designed to induce optimal levels of motivation and attention during task performance and induce a larger CNV wave. Results showed that the new tasks induced larger CNV waves and faster responses compared to the Traffic light task.

Next, sensitivity of the CNV to dopamine activity was studied. The study described in chapter 6 showed that methylphenidate dose dependently increased CNV amplitude and decreased response times. From these results it was concluded that CNV was a good candidate biomarker for dopamine activity. However, because methylphenidate affects not only dopamine but also noradrenaline neurotransmitter activity, pharmacological selectivity of the CNV still needed to be determined. To do this, the study in chapter 7 was conducted. CNV was measured in healthy volunteers under the influence of levodopa, a drug that selectively affects dopamine activity, and compared to a methylphenidate condition. This study showed that methylphenidate and levodopa had opposite effects on CNV amplitude and response time.

The results of the latter study seemed to challenge the conclusion of the study in chapter 6. CNV possesses characteristics that are important for a biomarker, including for example sensitivity, which was demonstrated by the fact that a low dose of methylphenidate evoked a measureable change in CNV amplitude and CNV results differentiated between doses. However, the studies described in this dissertation do not support the idea that the CNV is a biomarker which is selective for dopamine activity. On the other hand, taking into account that the levodopa dose in the final experiment was rather low, the results do not allow the conclusion that dopamine is not involved in CNV. 
Considering the literature on dopamine and CNV (e.g. (Amabile, et al., 1986; Oishi, et al., 1995; Tecce, 1991; Tecce, et al., 1975)), it is likely that dopamine does play a role in CNV. However, the findings described in the present dissertation provide insufficient support to claim a role of the CNV as a dopaminergic biomarker because the evidence was limited by the fact that methylphenidate is not a pure dopaminergic drug and that the attempt to show that the effects were specifically dopamine dependent failed. This issue may be addressed in future research.

\section{Methodological considerations}

A crucial aspect of our studies on dopaminergic influences on cognitive function and the CNV wave was the choice of the dopaminergic manipulations. In order to study a dose-response relationship we needed to find a drug that had a wide safe dose range. Methylphenidate fulfilled this requirement and is generally well tolerated. A drawback of employing methylphenidate in our studies was that it blocks the reuptake of both dopamine and noradrenaline (Hannestad, et al., 2010; Kuczenski \& Segal, 1997; Volkow, Wang, Fowler, Gatley, et al., 1998). Methylphenidate's potency may be higher for the noradrenaline- than the dopamine transporter (Kuczenski \& Segal, 1997), but dopamine is also transported by the noradrenaline transporter (Hannestad, et al., 2010; Pacholczyk, et al., 1991). Therefore it could not be deduced whether effects of methylphenidate on cognition and the CNV wave were mediated by dopamine, noradrenaline or a combination of the two.

In order to disentangle dopaminergic and noradrenergic effects, methylphenidate should have been contrasted with a pure dopaminergic- and a pure noradrenergic manipulation, ideally with the same mechanism. Due to the lack of respective specificity of dopamine and noradrenaline to the dopamine- and noradrenaline transporters (Hannestad, et al., 2010; Pacholczyk, et al., 1991), reuptake inhibiting drugs were not an option and other drugs had to be considered. Available noradrenergic drugs, including for example clonidine, guanfacine, idazoxan and propranolol were disregarded for various reasons such as lack of specificity or safety, or adverse side effects that would interfere with task performance (Currie, Lewis, McDevitt, Nicholson, \& Wright, 1988; Jakala, et al., 1999; Middleton, Sharma, Agouzoul, Sahakian, \& Robbins, 1999). Due to lack of availability of an appropriate purely noradrenergic drug, methylphenidate effects were thus only compared to a dopaminergic manipulation. As the precursor of dopamine, levodopa was the most purely dopaminergic drug available. However, even levodopa might not only affect dopamine but also noradrenaline, as noradrenaline is synthesized from dopamine (Breitenstein, Floel, et al., 2006; Julien, 2011). In 
fact, it might not be feasible to completely disentangle dopamine- from noradrenaline mediated effects. Given that these two neurotransmitters originate from common precursors and the close interactions between dopamine and noradrenaline, the practical relevance of disentangling their effects might actually be limited. In future research, investigators may consider if their research should focus on 'catecholamines' rather than dopamine or noradrenaline alone.

Another issue related to the choice of levodopa as a dopaminergic drug is that the mechanism by which it affects dopamine activity differs from that of methylphenidate. While levodopa has the potential to enhance presynaptic dopamine availability and thus may affect both tonic and phasic dopaminergic processes, methylphenidate does not have a direct releasing action (Breitenstein, Korsukewitz, et al., 2006; Seeman \& Madras, 2002). Low doses of dopamine agonists may even reduce phasic dopamine signals since phasic dopamine activity is inhibited by tonic dopamine activity on postsynaptic receptors and autoreceptors (Breitenstein, Korsukewitz, et al., 2006; Seeman \& Madras, 2002). A higher dose overcomes such an effect and causes generalized stimulation of the nervous system (Seeman \& Madras, 2002).

As the levodopa dose used in the studies in this dissertation was relatively low in order to avoid sedation, dopamine activity at the autoreceptors may have reduced the impact of phasic dopamine signals in our experiments. Combined influences of levodopa on tonic and phasic dopamine activity may have thus resulted in a net null effect of levodopa on dopamine availability. Such a supposition could be tested by assessment of prolactin in blood as prolactin levels decrease under the influence of increased dopamine availability. However, in our studies we administered domperidon, a peripheral dopamine antagonist, along with the levodopa treatment to prevent nausea. An increase in prolactin level induced by levodopa would thus be counteracted by domperidon (Brouwers, Assies, Wiersinga, Huizing, \& Tytgat, 1980).

Besides choice of drug, there was another methodological issue that was raised in this dissertation. The study procedures described in chapters 2 and 6 involved 8 hours of almost continuous performance of cognitive tasks on a computer. In contrast, participants in the studies described in chapters 3 and 7 were only involved in 1.5 hours of cognitive testing and could relax for approximately two hours before testing started. These circumstances may have contributed to performance on the cognitive tests. Participants subjected to 8 hours of testing may have been more tired than participants involved in 1.5 hours of testing. In the former situation, it is not unlikely that fatigue was induced in the participants and we may have unintentionally 
tested the influence of methylphenidate on cognitive performance under fatigue. This may be especially true for tests scheduled later on the day, including the word learning test. The differences between the designs of these studies limit the comparability of the results. On the other hand, these differences may have provided us with the key factor determining the cognitive enhancing potential of methylphenidate.

\section{Conclusions and implications}

The cognition enhancing effects of methylphenidate suggest that dopamine plays a role in cognitive functions such as verbal learning and memory, working memory, attention and speed of processing. However, the evidence is limited by the fact that methylphenidate is not a pure dopaminergic drug and that the attempt to show that the effects were specifically dopamine dependent failed. This issue also played a role in the validation of the CNV as a dopaminergic biomarker. CNV possesses characteristics that are important for a biomarker, but the findings described in the present dissertation provide insufficient support to claim a role of the CNV as a biomarker that is specific for dopaminergic activity.

The research described in this thesis highlights the impact of the experimental design and study procedures on the outcome of a study. Together, the results of the different studies employing different designs and procedures, but testing the same substance, suggest that healthy individuals may particularly benefit from methylphenidate's enhancing effects under challenging circumstances. Based on these results and considering that the use of methylphenidate to enhance study performance is currently common practice among university students (Greely, et al., 2008), future research may target practical applications of methylphenidate. For example, future studies may investigate if methylphenidate affects studying under pressure of an important exam, if it affects exam score and the time frame of such effects. 


\section{References}

Advokat, C. (2010). What are the cognitive effects of stimulants medications? Emphasis on adults with attention-deficit/hyperactivity disorder (ADHD). Neurosci Biobehav Rev, 34, 1256-1266.

Agay, N., Yechiam, E., Carmel, Z., \& Levkovitz, Y. (2010). Non-specific effects of methylphenidate (Ritalin) on cognitive ability and decisionmaking of ADHD and healthy adults. Psychopharmacology (Berl), 210(4), 511-519.

Amabile, G., Fattapposta, F., Pozzessere, G., Albani, G., Sanarelli, L., Rizzo, P. A., et al. (1986). Parkinson disease: electrophysiological (CNV) analysis related to pharmacological treatment. Electroencephalogr Clin Neurophysiol, 64(6), 521-524.

Aman, M. G., Vamos, M., \& Werry, J. S. (1984). Effects of methylphenidate in normal adults with reference to drug action in hyperactivity. Aust N Z J Psychiatry, 18(1), 86-88.

Anderer, P., Saletu, B., Semlitsch, H. V., \& Pascual-Marqui, R. D. (2002). Perceptual and cognitive event-related potentials in neuropsychopharmacology: methodological aspects and clinical applications (pharmaco-ERP topography and tomography). Methods Find Exp Clin Pharmacol, 24 Suppl C, 121-137.

Andreu, N., Chale, J. J., Senard, J. M., Thalamas, C., Montastruc, J. L., \& Rascol, O. (1999). L-Dopa-induced sedation: a double-blind cross-over controlled study versus triazolam and placebo in healthy volunteers. Clinical neuropharmacology, 22(1), 15-23.

Arnsten, A. F., \& Dudley, A. G. (2005). Methylphenidate improves prefrontal cortical cognitive function through alpha2 adrenoceptor and dopamine D1 receptor actions: Relevance to therapeutic effects in Attention Deficit Hyperactivity Disorder. Behav Brain Funct, 1(1), 2.

Ashton, H., Marsh, V. R., Millman, J. E., Rawlins, M. D., Telford, R., \& Thompson, J. W. (1980). Biphasic dose-related responses of the CNV (contingent negative variation) to I.V. nicotine in man. $\mathrm{Br} \mathrm{J} \mathrm{Clin}$ Pharmacol, 10(6), 579-589.

Ashton, H., Millman, J. E., Telford, R., \& Thompson, J. W. (1974). The effect of caffeine, nitrazepam and cigarette smoking on the contingent negative variation in man. Electroencephalogr Clin Neurophysiol, 37(1), 59-71.

Ashton, H., Millman, J. E., Telford, R., \& Thompson, J. W. (1976). A comparison of some physiological and psychological effects of 
propranolol and diazepam in normal subjects. Br J Clin Pharmacol(3), 551-559.

Ashton, H., \& Rawlins, M. D. (1978). Central nervous system depressant actions of clonidine and UK-14,304: partial dissociation of EEG and behavioural effects. Br J Clin Pharmacol, 5(2), 135-140.

Azuma, T., Cruz, R. F., Bayles, K. A., Tomoeda, C. K., \& Montgomery, E. B., Jr. (2003). A longitudinal study of neuropsychological change in individuals with Parkinson's disease. Int J Geriatr Psychiatry, 18(11), 1043-1049.

Babiloni, C., Brancucci, A., Arendt-Nielsen, L., Del Percio, C., Babiloni, F., Pascual-Marqui, R. D., et al. (2004). Cortical sensorimotor interactions during the expectancy of a go/no-go task: effects of painful stimuli. Behav Neurosci, 118(5), 925-935.

Babiloni, C., Brancucci, A., Capotosto, P., Romani, G. L., Arendt-Nielsen, L., Chen, A. C., et al. (2005). Slow cortical potential shifts preceding sensorimotor interactions. Brain Res Bull, 65(4), 309-316.

Baddeley, A. (1992). Working memory. Science, 255(5044), 556-559.

Banaschewski, T., \& Brandeis, D. (2007). Annotation: what electrical brain activity tells us about brain function that other techniques cannot tell us - a child psychiatric perspective. J Child Psychol Psychiatry, 48(5), 415-435.

Bares, M., \& Rektor, I. (2001). Basal ganglia involvement in sensory and cognitive processing. A depth electrode CNV study in human subjects. Clin Neurophysiol, 112(11), 2022-2030.

Bares, M., Rektor, I., Kanovsky, P., \& Streitova, H. (2003). Cortical and subcortical distribution of middle and long latency auditory and visual evoked potentials in a cognitive (CNV) paradigm. Clin Neurophysiol, 114(12), 2447-2460.

Barrett, S. P., \& Leyton, M. (2004). Acute phenylalanine/tyrosine depletion: A new method to study the role of catecholamines in psychiatric disorders. Primary Psychiatry, 11, 37-41.

Ben-Itzhak, R., Giladi, N., Gruendlinger, L., \& Hausdorff, J. M. (2008). Can methylphenidate reduce fall risk in community-living older adults? A double-blind, single-dose cross-over study. J Am Geriatr Soc, 56(4), 695-700.

Ben-Jonathan, N. (1985). Dopamine: a prolactin-inhibiting hormone. Endocr Rev, 6(4), 564-589.

Bishop, C., Roehrs, T., Rosenthal, L., \& Roth, T. (1997). Alerting effects of methylphenidate under basal and sleep-deprived conditions. Exp Clin Psychopharmacol, 5(4), 344-352. 
Bokura, H., Yamaguchi, S., \& Kobayashi, S. (2001). Electrophysiological correlates for response inhibition in a Go/NoGo task. Clin Neurophysiol, 112(12), 2224-2232.

Bond, A., \& Lader, M. (1974). The use of analogue scales in rating subjective feelings. British Journal of Medical Psychology, 47, 211-218.

Bongiovanni, R., Newbould, E., \& Jaskiw, G. E. (2008). Tyrosine depletion lowers dopamine synthesis and desipramine-induced prefrontal cortex catecholamine levels. Brain Res, 1190, 39-48.

Bray, C. L., Cahill, K. S., Oshier, J. T., Peden, C. S., Theriaque, D. W., Flotte, T. R., et al. (2004). Methylphenidate does not improve cognitive function in healthy sleep-deprived young adults. J Investig Med, 52(3), 192-201.

Breitenstein, C., Floel, A., Korsukewitz, C., Wailke, S., Bushuven, S., \& Knecht, S. (2006). A shift of paradigm: from noradrenergic to dopaminergic modulation of learning? Journal of the neurological sciences, 248(1-2), 42-47.

Breitenstein, C., Korsukewitz, C., Floel, A., Kretzschmar, T., Diederich, K., \& Knecht, S. (2006). Tonic dopaminergic stimulation impairs associative learning in healthy subjects. Neuropsychopharmacology : official publication of the American College of Neuropsychopharmacology, 31(11), 2552-2564.

Brignell, C. M., \& Curran, H. V. (2006). Drugs, sweat, and fears: a comparison of the effects of diazepam and methylphenidate on fear conditioning. Psychopharmacology (Berl), 186(4), 504-516.

Brignell, C. M., Rosenthal, J., \& Curran, H. V. (2007). Pharmacological manipulations of arousal and memory for emotional material: effects of a single dose of methylphenidate or lorazepam. J Psychopharmacol, 21(7), 673-683.

Brouwers, J. R., Assies, J., Wiersinga, W. M., Huizing, G., \& Tytgat, G. N. (1980). Plasma prolactin levels after acute and subchronic oral administration of domperidone and of metoclopramide: a cross-over study in healthy volunteers. Clinical endocrinology, 12(5), 435-440.

Brumaghim, J. T., \& Klorman, R. (1998). Methylphenidate's effects on pairedassociate learning and event-related potentials of young adults. Psychophysiology, 35(1), 73-85.

Brumaghim, J. T., Klorman, R., Strauss, J., Lewine, J. D., \& Goldstein, M. G. (1987). Does methylphenidate affect information processing? Findings from two studies on performance and P3b latency. Psychophysiology, 24(3), 361-373.

Brunia, C. H. (1988). Movement and stimulus preceding negativity. Biol Psychol, 26(1-3), 165-178. 
Brunia, C. H., \& van Boxtel, G. J. (2001). Wait and see. Int J Psychophysiol, 43(1), 59-75.

Bullmore, E., Suckling, J., Zelaya, F., Long, C., Honey, G., Reed, L., et al. (2003). Practice and difficulty evoke anatomically and pharmacologically dissociable brain activation dynamics. Cereb Cortex, 13(2), 144-154.

Butler, S. R., \& Glass, A. (1974). Asymmetries in the CNV over left and right hemispheres while subjects await numeric information. Biol Psychol, 2(1), 1-16.

Callaway, E. (1984). Human information-processing: some effects of methylphenidate, age, and scopolamine. Biol Psychiatry, 19(5), 649662.

Camp-Bruno, J. A., \& Herting, R. L. (1994). Cognitive effects of milacemide and methylphenidate in healthy young adults. Psychopharmacology (Berl), 115(1-2), 46-52.

Chamberlain, S. R., Muller, U., Blackwell, A. D., Robbins, T. W., \& Sahakian, B. J. (2006). Noradrenergic modulation of working memory and emotional memory in humans. Psychopharmacology, 188(4), 397-407.

Chan, S. C., Chan, C. C., Kwan, A. S., Ting, K. H., \& Chui, T. Y. (2012). Orienting attention modulates pain perception: an ERP study. PLoS ONE, 7(6), e40215.

Chana, P., Fierro, A., Reyes-Parada, M., \& Saez-Briones, P. (2003). Pharmacokinetic comparison of Sinemet and Grifoparkin (levodopa/carbidopa 250/25 mg) in Parkinson s disease: a single dose study. Revista medica de Chile, 131(6), 623-631.

Clark, C. R., Geffen, G. M., \& Geffen, L. B. (1986a). Role of monoamine pathways in attention and effort: effects of clonidine and methylphenidate in normal adult humans. Psychopharmacology (Berl), 90(1), 35-39.

Clark, C. R., Geffen, G. M., \& Geffen, L. B. (1986b). Role of monoamine pathways in the control of attention: effects of droperidol and methylphenidate in normal adult humans. Psychopharmacology (Berl), 90(1), 28-34.

Clatworthy, P. L., Lewis, S. J., Brichard, L., Hong, Y. T., Izquierdo, D., Clark, L., et al. (2009). Dopamine release in dissociable striatal subregions predicts the different effects of oral methylphenidate on reversal learning and spatial working memory. J Neurosci, 29(15), 4690-4696.

Cools, R. (2006). Dopaminergic modulation of cognitive function-implications for L-DOPA treatment in Parkinson's disease. Neuroscience and biobehavioral reviews, 30(1), 1-23. 
Cools, R. (2008). Role of dopamine in the motivational and cognitive control of behavior. The Neuroscientist : a review journal bringing neurobiology, neurology and psychiatry, 14(4), 381-395.

Cools, R., Barker, R. A., Sahakian, B. J., \& Robbins, T. W. (2001). Enhanced or impaired cognitive function in Parkinson's disease as a function of dopaminergic medication and task demands. Cerebral cortex, 11(12), 1136-1143.

Cools, R., \& Robbins, T. W. (2004). Chemistry of the adaptive mind. Philos Transact A Math Phys Eng Sci, 362(1825), 2871-2888.

Coons, H. W., Peloquin, L. J., Klorman, R., Bauer, L. O., Ryan, R. M., Perlmutter, R. A., et al. (1981). Effect of methylphenidate on young adult's vigilance and event-related potentials. Electroencephalogr Clin Neurophysiol, 51(4), 373-387.

Cooper, N. J., Keage, H., Hermens, D., Williams, L. M., Debrota, D., Clark, C. R., et al. (2005). The dose-dependent effect of methylphenidate on performance, cognition and psychophysiology. J Integr Neurosci, 4(1), 123-144.

Costa, A., Peppe, A., Dell'Agnello, G., Carlesimo, G. A., Murri, L., Bonuccelli, U., et al. (2003). Dopaminergic modulation of visual-spatial working memory in Parkinson's disease. Dement Geriatr Cogn Disord, 15(2), 55-66.

Coull, J. T. (1998). Neural correlates of attention and arousal: insights from electrophysiology, functional neuroimaging and psychopharmacology. Prog Neurobiol, 55(4), 343-361.

Coull, J. T., Frith, C. D., Dolan, R. J., Frackowiak, R. S., \& Grasby, P. M. (1997). The neural correlates of the noradrenergic modulation of human attention, arousal and learning. The European journal of neuroscience, 9(3), 589-598.

Currie, D., Lewis, R. V., McDevitt, D. G., Nicholson, A. N., \& Wright, N. A. (1988). Central effects of beta-adrenoceptor antagonists. I-Performance and subjective assessments of mood. British journal of clinical pharmacology, 26(2), 121-128.

Dickinson, D., Ragland, J. D., Calkins, M. E., Gold, J. M., \& Gur, R. C. (2006). A comparison of cognitive structure in schizophrenia patients and healthy controls using confirmatory factor analysis. Schizophr Res, 85(1-3), 20-29.

Diller, L. H. (1996). The run on Ritalin. Attention deficit disorder and stimulant treatment in the 1990s. Hastings Cent Rep, 26(2), 12-18.

Dodds, C., Muller, U., \& Manly, T. (2008). Effects of Psychostimulants on Alertness and Spatial Bias in Healthy Participants. J Cogn Neurosci. 
Dodds, C. M., Muller, U., Clark, L., van Loon, A., Cools, R., \& Robbins, T. W. (2008). Methylphenidate has differential effects on blood oxygenation level-dependent signal related to cognitive subprocesses of reversal learning. J Neurosci, 28(23), 5976-5982.

Dujardin, K., \& Laurent, B. (2003). Dysfunction of the human memory systems: role of the dopaminergic transmission. Curr Opin Neurol, 16 Suppl 2, S11-16.

Ellenbogen, M. A., Young, S. N., Dean, P., Palmour, R. M., \& Benkelfat, C. (1996). Mood response to acute tryptophan depletion in healthy volunteers: sex differences and temporal stability. Neuropsychopharmacology, 15(5), 465-474.

Elliott, R., Sahakian, B. J., Matthews, K., Bannerjea, A., Rimmer, J., \& Robbins, T. W. (1997). Effects of methylphenidate on spatial working memory and planning in healthy young adults. Psychopharmacology (Berl), 131(2), 196-206.

Ellis, K. A., Mehta, M. A., Naga Venkatesha Murthy, P. J., McTavish, S. F., Nathan, P. J., \& Grasby, P. M. (2007). Tyrosine depletion alters cortical and limbic blood flow but does not modulate spatial working memory performance or task-related blood flow in humans. Hum Brain Mapp, 28(11), 1136-1149.

Evans, R. W., Gualtieri, C. T., \& Amara, I. (1986). Methylphenidate and memory: dissociated effects in hyperactive children. Psychopharmacology (Berl), 90(2), 211-216.

Falkenstein, M., Koshlykova, N. A., Kiroj, V. N., Hoormann, J., \& Hohnsbein, J. (1995). Late ERP components in visual and auditory Go/Nogo tasks. Electroencephalogr Clin Neurophysiol, 96(1), 36-43.

Fan, J., Kolster, R., Ghajar, J., Suh, M., Knight, R. T., Sarkar, R., et al. (2007). Response anticipation and response conflict: an event-related potential and functional magnetic resonance imaging study. $J$ Neurosci, 27(9), 2272-2282.

Farber, D. A., Beteleva, T. G., \& Ignat'eva, I. S. (2004). Functional organization of the brain during the operation of the working memory. Fiziol Cheloveka, 30(2), 5-12.

Fillmore, M. T., \& Rush, C. R. (2002). Impaired inhibitory control of behavior in chronic cocaine users. Drug Alcohol Depend, 66(3), 265-273.

Finke, K., Dodds, C. M., Bublak, P., Regenthal, R., Baumann, F., Manly, T., et al. (2010). Effects of modafinil and methylphenidate on visual attention capacity: a TVA-based study. Psychopharmacology, 210(3), 317-329.

Fitzpatrick, P., Klorman, R., Brumaghim, J. T., \& Keefover, R. W. (1988). Effects of methylphenidate on stimulus evaluation and response 
processes: evidence from performance and event-related potentials. Psychophysiology, 25(3), 292-304.

Floel, A., Breitenstein, C., Hummel, F., Celnik, P., Gingert, C., Sawaki, L., et al. (2005). Dopaminergic influences on formation of a motor memory. Annals of neurology, 58(1), 121-130.

Floel, A., Garraux, G., Xu, B., Breitenstein, C., Knecht, S., Herscovitch, P., et al. (2008). Levodopa increases memory encoding and dopamine release in the striatum in the elderly. Neurobiology of aging, 29(2), 267-279.

Forster, B., \& Pavone, E. F. (2008). Electrophysiological correlates of crossmodal visual distractor congruency effects: evidence for response conflict. Cogn Affect Behav Neurosci, 8(1), 65-73.

Friedman, D., \& Johnson, R., Jr. (2000). Event-related potential (ERP) studies of memory encoding and retrieval: a selective review. Microsc Res Tech, 51(1), 6-28.

Gaillard, A. W. (1976). Effects of warning-signal modality on the contingent negative variation (CNV). Biological psychology, 4(2), 139-154.

Gajewski, P. D., Stoerig, P., \& Falkenstein, M. (2008). ERP--correlates of response selection in a response conflict paradigm. Brain Res, 1189, 127-134.

Garcia-Larrea, L., \& Cezanne-Bert, G. (1998). P3, positive slow wave and working memory load: a study on the functional correlates of slow wave activity. Electroencephalogr Clin Neurophysiol, 108(3), 260-273.

Genderson, M. R., Dickinson, D., Diaz-Asper, C. M., Egan, M. F., Weinberger, D. R., \& Goldberg, T. E. (2007). Factor analysis of neurocognitive tests in a large sample of schizophrenic probands, their siblings, and healthy controls. Schizophr Res, 94(1-3), 231-239.

Gevins, A., Smith, M. E., \& McEvoy, L. K. (2002). Tracking the cognitive pharmacodynamics of psychoactive substances with combinations of behavioral and neurophysiological measures. Neuropsychopharmacology : official publication of the American College of Neuropsychopharmacology, 26(1), 27-39.

Ghilardi, M. F., Feigin, A. S., Battaglia, F., Silvestri, G., Mattis, P., Eidelberg, D., et al. (2007). L-Dopa infusion does not improve explicit sequence learning in Parkinson's disease. Parkinsonism \& related disorders, 13(3), 146-151.

Gijsman, H. J., Scarna, A., Harmer, C. J., McTavish, S. B., Odontiadis, J., Cowen, P. J., et al. (2002). A dose-finding study on the effects of branch chain amino acids on surrogate markers of brain dopamine function. Psychopharmacology (Berl), 160(2), 192-197. 
Golob, E. J., \& Starr, A. (2000). Effects of stimulus sequence on event-related potentials and reaction time during target detection in Alzheimer's disease. Clinical neurophysiology: official journal of the International Federation of Clinical Neurophysiology, 111(8), 1438-1449.

Greely, H., Sahakian, B., Harris, J., Kessler, R. C., Gazzaniga, M., Campbell, P., et al. (2008). Towards responsible use of cognitive-enhancing drugs by the healthy. Nature, 456(7223), 702-705.

Grevet, E. H., Tietzmann, M. R., Shansis, F. M., Hastenpflugl, C., Santana, L. C., Forster, L., et al. (2002). Behavioural effects of acute phenylalanine and tyrosine depletion in healthy male volunteers. $J$ Psychopharmacol, 16(1), 51-55.

Haagh, S. A., \& Brunia, C. H. (1985). Anticipatory response-relevant muscle activity, CNV amplitude and simple reaction time. Electroencephalogr Clin Neurophysiol, 61(1), 30-39.

Halliday, R., Callaway, E., Naylor, H., Gratzinger, P., \& Prael, R. (1986). The effects of stimulant drugs on information processing in elderly adults. J Gerontol, 41(6), 748-757.

Hannestad, J., Gallezot, J. D., Planeta-Wilson, B., Lin, S. F., Williams, W. A., van Dyck, C. H., et al. (2010). Clinically relevant doses of methylphenidate significantly occupy norepinephrine transporters in humans in vivo. Biol Psychiatry, 68(9), 854-860.

Hansenne, M., Pitchot, W., Pinto, E., Papart, P., \& Ansseau, M. (2000). Serotonergic-1a activity and contingent negative variation. Biol Psychol, 52(3), 259-265.

Harmer, C. J., McTavish, S. F., Clark, L., Goodwin, G. M., \& Cowen, P. J. (2001). Tyrosine depletion attenuates dopamine function in healthy volunteers. Psychopharmacology (Berl), 154(1), 105-111.

Harrison, B. J., Olver, J. S., Norman, T. R., Burrows, G. D., Wesnes, K. A., \& Nathan, P. J. (2004). Selective effects of acute serotonin and catecholamine depletion on memory in healthy women. $J$ Psychopharmacol, 18(1), 32-40.

Hermens, D. F., Cooper, N. J., Clark, C. R., Debrota, D., Clarke, S. D., \& Williams, L. M. (2007). An integrative approach to determine the best behavioral and biological markers of methylphenidate. $J$ Integr Neurosci, 6(1), 105-140.

Hink, R. F., Fenton, W. H., Jr., Tinklenberg, J. R., Pfefferbaum, A., \& Kopell, B. S. (1978). Vigilance and human attention under conditions of methylphenidate and secobarbital intoxication: an assessment using brain potentials. Psychophysiology, 15(2), 116-125.

Husain, M., \& Mehta, M. A. (2011). Cognitive enhancement by drugs in health and disease. Trends Cogn Sci, 15(1), 28-36. 
Ikeda, A., Shibasaki, H., Kaji, R., Terada, K., Nagamine, T., Honda, M., et al. (1997). Dissociation between contingent negative variation (CNV) and Bereitschaftspotential (BP) in patients with parkinsonism. Electroencephalography and clinical neurophysiology, 102(2), 142151.

Iversen, S. D., \& Iversen, L. L. (2007). Dopamine: 50 years in perspective. Trends Neurosci, 30(5), 188-193.

Izquierdo, I., Bevilaqua, L. R., Rossato, J. I., Lima, R. H., Medina, J. H., \& Cammarota, M. (2008). Age-dependent and age-independent human memory persistence is enhanced by delayed posttraining methylphenidate administration. Proc Natl Acad Sci U S A, 105(49), 19504-19507.

Jadad, A. R., Moore, R. A., Carroll, D., Jenkinson, C., Reynolds, D. J., Gavaghan, D. J., et al. (1996). Assessing the quality of reports of randomized clinical trials: is blinding necessary? Control Clin Trials, 17(1), 1-12.

Jakala, P., Riekkinen, M., Sirvio, J., Koivisto, E., Kejonen, K., Vanhanen, M., et al. (1999). Guanfacine, but not clonidine, improves planning and working memory performance in humans. Neuropsychopharmacology : official publication of the American College of Neuropsychopharmacology, 20(5), 460-470.

James, W. (1890). The Principles of Psychology New York Henry Holt.

Jaskowski, P., \& Verleger, R. (1993). A clock paradigm to study the relationship between expectancy and response force. Perceptual and motor skills, 77(1), 163-174.

Jasper, H. H. (1958). The ten-twenty electrode system of the International Federation. Electroencephalogr. Clin. Neurophysiol., 10, 370-375.

Julien, R. M. (2005). A primer of drug addiction. A comprehensive guide to the actions, uses and side effects of psychoactive drugs (10th ed.). New York: Worth Publishers.

Julien, R. M. (2011). A primer of drug addiction. A comprehensive guide to the actions, uses, and side effects of psychoactive drugs (12th ed.). New York: Worth Publishers.

Kessels, R. P., Postma, A., \& de Haan, E. H. (1999). Object Relocation: a program for setting up, running, and analyzing experiments on memory for object locations. Behav Res Methods Instrum Comput, 31(3), 423-428.

Kessels, R. P., Postma, A., Wester, A. J., \& de Haan, E. H. (2000). Memory for object locations in Korsakoff's amnesia. Cortex, 36(1), 47-57. 
Klaassen, T., Riedel, W. J., Deutz, N. E., \& Van Praag, H. M. (2002). Mood congruent memory bias induced by tryptophan depletion. Psychol Med, 32(1), 167-172.

Kleijnen, J., Knipschild, P., \& Riet ter, G. (1991). Clinical trials of homeopathy. BMJ, 302, 316-323.

Klorman, R., \& Bentsen, E. (1975). Effects of warning-signal duration on the early and late components of the contingent negative variation. Biol Psychol, 3(4), 263-275.

Knecht, S., Breitenstein, C., Bushuven, S., Wailke, S., Kamping, S., Floel, A., et al. (2004). Levodopa: faster and better word learning in normal humans. Annals of neurology, 56(1), 20-26.

Knott, V. J., \& Venables, P. H. (1980). Separate and combined effects of alcohol and tobacco on the amplitude of the contingent negative variation. Psychopharmacology, 70(2), 167-172.

Kobayashi, K., \& Yasoshima, Y. (2001). The central noradrenaline system and memory consolidation. The Neuroscientist : a review journal bringing neurobiology, neurology and psychiatry, 7(5), 371-376.

Kollins, S. H., Rush, C. R., Pazzaglia, P. J., \& Ali, J. A. (1998). Comparison of acute behavioral effects of sustained-release and immediate-release methylphenidate. Exp Clin Psychopharmacol, 6(4), 367-374.

Kopell, B. S., Wittner, W. K., Lunde, D. T., Wolcott, L. J., \& Tinklenberg, J. R. (1974). The effects of methamphetamine and secobarbital on the contingent negative variation amplitude. Psychopharmacologia, 34(1), $55-62$.

Kratz, O., Diruf, M. S., Studer, P., Gierow, W., Buchmann, J., Moll, G. H., et al. (2009). Effects of methylphenidate on motor system excitability in a response inhibition task. Behav Brain Funct, 5, 12.

Kuczenski, R., \& Segal, D. S. (1997). Effects of methylphenidate on extracellular dopamine, serotonin, and norepinephrine: comparison with amphetamine. Journal of neurochemistry, 68(5), 2032-2037.

Kulisevsky, J. (2000). Role of dopamine in learning and memory: implications for the treatment of cognitive dysfunction in patients with Parkinson's disease. Drugs \& aging, 16(5), 365-379.

Kupietz, S. S., Richardson, E., Gadow, K. D., \& Winsberg, B. G. (1980). Effects of methylphenidate on learning a 'beginning reading vocabulary' by normal adults. Psychopharmacology (Berl), 69(1), 69-72.

Kuypers, K. P., \& Ramaekers, J. G. (2005). Transient memory impairment after acute dose of $75 \mathrm{mg}$ 3.4-Methylene-dioxymethamphetamine. $J$ Psychopharmacol, 19(6), 633-639.

Kuypers, K. P., \& Ramaekers, J. G. (2007). Acute dose of MDMA (75 mg) impairs spatial memory for location but leaves contextual processing 
of visuospatial information unaffected. Psychopharmacology (Berl), 189(4), 557-563.

Landau, S. M., Lal, R., O'Neil, J. P., Baker, S., \& Jagust, W. J. (2008). Striatal Dopamine and Working Memory. Cereb Cortex.

Larriviere, D., Williams, M. A., Rizzo, M., \& Bonnie, R. J. (2009). Responding to requests from adult patients for neuroenhancements: guidance of the Ethics, Law and Humanities Committee. Neurology, 73(17), 14061412.

Le Masurier, M., Cowen, P. J., \& Sharp, T. (2004). Fos immunocytochemical studies on the neuroanatomical sites of action of acute tyrosine depletion in the rat brain. Psychopharmacology (Berl), 171(4), 435440.

Le Masurier, M., Houston, G., Cowen, P., Grasby, T. S., \& Hume, S. (2004). Tyrosine-Free Amino Acid Mixture Attenuates Amphetamine-Induced Displacement of [11C]Raclopride in Striatum In Vivo: A Rat PET Study. Synapse, 51, 151-157.

Lefebvre, C. D., Marchand, Y., Eskes, G. A., \& Connolly, J. F. (2005). Assessment of working memory abilities using an event-related brain potential (ERP)-compatible digit span backward task. Clin Neurophysiol, 116(7), 1665-1680.

Leonard, B. E., McCartan, D., White, J., \& King, D. J. (2004). Methylphenidate: a review of its neuropharmacological, neuropsychological and adverse clinical effects. Hum Psychopharmacol, 19(3), 151-180.

Levy, F. (2009). Dopamine vs noradrenaline: inverted-U effects and ADHD theories. Aust N Z J Psychiatry, 43(2), 101-108.

Lewis, S. J., Slabosz, A., Robbins, T. W., Barker, R. A., \& Owen, A. M. (2005). Dopaminergic basis for deficits in working memory but not attentional set-shifting in Parkinson's disease. Neuropsychologia, 43(6), 823-832.

Leyton, M., Dagher, A., Boileau, I., Casey, K., Baker, G. B., Diksic, M., et al. (2004). Decreasing amphetamine-induced dopamine release by acute phenylalanine/tyrosine depletion: A PET/[11C]raclopride study in healthy men. Neuropsychopharmacology, 29(2), 427-432.

Leyton, M., Young, S. N., Pihl, R. O., Etezadi, S., Lauze, C., Blier, P., et al. (2000). Effects on mood of acute phenylalanine/tyrosine depletion in healthy women. Neuropsychopharmacology, 22(1), 52-63.

Lezak, M. D. (1995). Neuropsychological Assessment (3rd ed.). New York: Oxford University Press.

Linssen, A. M., Vuurman, E. F., Sambeth, A., Nave, S., Spooren, W., Vargas, G., et al. (2011). Contingent negative variation as a dopaminergic 
biomarker: evidence from dose-related effects of methylphenidate. Psychopharmacology, 218(3), 533-542.

Linssen, A. M., Vuurman, E. F., Sambeth, A., \& Riedel, W. J. (2012). Methylphenidate produces selective enhancement of declarative memory consolidation in healthy volunteers. Psychopharmacology, 221(4), 611-619.

Logan, G. D. (1994). On the ability to control thought and action: A user's guide to the stop signal paradigm. In D. Dagenbach \& T. H. Carr (Eds.), Inhibitory processes in attention, memory, and language. San Diego: Academic Press.

Loveless, N. E., \& Sanford, A. J. (1974a). Effects of age on the contingent negative variation and preparatory set in a reaction-time task. $J$ Gerontol, 29(1), 52-63.

Loveless, N. E., \& Sanford, A. J. (1974b). Slow potential correlates of preparatory set. Biol Psychol, 1(4), 303-314.

Loveless, N. E., \& Sanford, A. J. (1975). The impact of warning signal intensity on reaction time and components of the contingent negative variation. Biological psychology, 2(3), 217-226.

Luciana, M., \& Collins, P. F. (1997). Dopaminergic Modulation of Working Memory for Spatial but Not Object Cues in Normal Humans. Journal of Cognitive Neuroscience, 9(3), 330-347

Luciana, M., Collins, P. F., \& Depue, R. A. (1998). Opposing roles for dopamine and serotonin in the modulation of human spatial working memory functions. Cereb Cortex, 8(3), 218-226.

Luciana, M., Depue, R. A., Arbisi, P., \& Leon, A. (1992). Facilitation of Working Memory in Humans by a D2 Dopamine Receptor Agonist. Journal of Cognitive Neuroscience, 4(1), 58-68.

Luck, S. J. (2005). An introduction to the event-related potential technique. Cambridge: The MIT Press.

Luthringer, R., Rinaudo, G., Toussaint, M., Bailey, P., Muller, G., Muzet, A., et al. (1999). Electroencephalographic characterization of brain dopaminergic stimulation by apomorphine in healthy volunteers. Neuropsychobiology, 39(1), 49-56.

Lythe, K. E., Anderson, I. M., Deakin, J. F., Elliott, R., \& Strickland, P. L. (2005). Lack of behavioural effects after acute tyrosine depletion in healthy volunteers. J Psychopharmacol, 19(1), 5-11.

Maher, B. (2008). Poll results: look who's doping. Nature, 452(7188), 674-675.

Markou, A., Chiamulera, C., Geyer, M. A., Tricklebank, M., \& Steckler, T. (2009). Removing obstacles in neuroscience drug discovery: the future path for animal models. Neuropsychopharmacology : official 
publication of the American College of Neuropsychopharmacology, 34(1), 74-89.

Marsden, C. D., \& Parkes, J. D. (1977). Success and problems of long-term levodopa therapy in Parkinson's disease. Lancet, 1(8007), 345-349.

Matrenza, C., Hughes, J. M., Kemp, A. H., Wesnes, K. A., Harrison, B. J., \& Nathan, P. J. (2004). Simultaneous depletion of serotonin and catecholamines impairs sustained attention in healthy female subjects without affecting learning and memory. J Psychopharmacol, 18(1), 2131.

McCallum, W. C., Cooper, R., \& Pocock, P. V. (1988). Brain slow potential and ERP changes associated with operator load in a visual tracking task. Electroencephalogr Clin Neurophysiol, 69(5), 453-468.

McLean, A., Rubinsztein, J. S., Robbins, T. W., \& Sahakian, B. J. (2004). The effects of tyrosine depletion in normal healthy volunteers: implications for unipolar depression. Psychopharmacology (Berl), 171(3), 286-297.

McNair, D., Lorr, M., \& Droppleman, L. (1992). EdITS Manual for the Profile of Mood States. San Diego: Educational and Industrial Testing Service.

McTavish, S. F., Cowen, P. J., \& Sharp, T. (1999). Effect of a tyrosine-free amino acid mixture on regional brain catecholamine synthesis and release. Psychopharmacology (Berl), 141(2), 182-188.

McTavish, S. F., McPherson, M. H., Harmer, C. J., Clark, L., Sharp, T., Goodwin, G. M., et al. (2001). Antidopaminergic effects of dietary tyrosine depletion in healthy subjects and patients with manic illness. Br J Psychiatry, 179, 356-360.

Mecklinger, A. (2010). The control of long-term memory: brain systems and cognitive processes. Neuroscience and biobehavioral reviews, 34(7), 1055-1065.

Meeter, M., Talamini, L., Schmitt, J. A., \& Riedel, W. J. (2006). Effects of 5HT on memory and the hippocampus: model and data. Neuropsychopharmacology, 31(4), 712-720.

Mehta, M. A., Goodyer, I. M., \& Sahakian, B. J. (2004). Methylphenidate improves working memory and set-shifting in AD/HD: relationships to baseline memory capacity. J Child Psychol Psychiatry, 45(2), 293-305.

Mehta, M. A., Gumaste, D., Montgomery, A. J., McTavish, S. F., \& Grasby, P. M. (2005). The effects of acute tyrosine and phenylalanine depletion on spatial working memory and planning in healthy volunteers are predicted by changes in striatal dopamine levels. Psychopharmacology (Berl), 180(4), 654-663.

Mehta, M. A., Hinton, E. C., Montgomery, A. J., Bantick, R. A., \& Grasby, P. M. (2005). Sulpiride and mnemonic function: effects of a dopamine D2 
receptor antagonist on working memory, emotional memory and longterm memory in healthy volunteers. J Psychopharmacol, 19(1), 29-38.

Mehta, M. A., Owen, A. M., Sahakian, B. J., Mavaddat, N., Pickard, J. D., \& Robbins, T. W. (2000). Methylphenidate enhances working memory by modulating discrete frontal and parietal lobe regions in the human brain. J Neurosci, 20(6), RC65.

Mehta, M. A., \& Riedel, W. J. (2006). Dopaminergic enhancement of cognitive function. Curr Pharm Des, 12(20), 2487-2500.

Mehta, M. A., Sahakian, B. J., McKenna, P. J., \& Robbins, T. W. (1999). Systemic sulpiride in young adult volunteers simulates the profile of cognitive deficits in Parkinson's disease. Psychopharmacology (Berl), $146(2), 162-174$.

Micallef-Roll, J., Rihet, P., Hasbroucq, T., Possamai, C., \& Blin, O. (2001). Levodopa-induced drowsiness in healthy volunteers: results of a choice reaction time test combined with a subjective evaluation of sedation. Clinical neuropharmacology, 24(2), 91-94.

Middleton, H. C., Sharma, A., Agouzoul, D., Sahakian, B. J., \& Robbins, T. W. (1999). Idazoxan potentiates rather than antagonizes some of the cognitive effects of clonidine. Psychopharmacology, 145(4), 401-411.

Missale, C., Nash, S. R., Robinson, S. W., Jaber, M., \& Caron, M. G. (1998). Dopamine receptors: from structure to function. Physiological reviews, 78(1), 189-225.

Montgomery, A. J., McTavish, S. F., Cowen, P. J., \& Grasby, P. M. (2003). Reduction of brain dopamine concentration with dietary tyrosine plus phenylalanine depletion: an [11C]raclopride PET study. The American journal of psychiatry, 160(10), 1887-1889.

Moustafa, A. A., Sherman, S. J., \& Frank, M. J. (2008). A dopaminergic basis for working memory, learning and attentional shifting in Parkinsonism. Neuropsychologia, 46(13), 3144-3156.

Mulder, E. J., Linssen, W. H., \& de Geus, E. I. (2002). Reduced sensory anticipation in migraine. Psychophysiology, 39(2), 166-174.

Muller, J., Dreisbach, G., Goschke, T., Hensch, T., Lesch, K. P., \& Brocke, B. (2007). Dopamine and cognitive control: the prospect of monetary gains influences the balance between flexibility and stability in a setshifting paradigm. Eur J Neurosci, 26(12), 3661-3668.

Muller, U., Suckling, J., Zelaya, F., Honey, G., Faessel, H., Williams, S. C., et al. (2005). Plasma level-dependent effects of methylphenidate on task-related functional magnetic resonance imaging signal changes. Psychopharmacology (Berl), 180(4), 624-633.

Nagai, T., Takuma, K., Kamei, H., Ito, Y., Nakamichi, N., Ibi, D., et al. (2007). Dopamine D1 receptors regulate protein synthesis-dependent 
long-term recognition memory via extracellular signal-regulated kinase 1/2 in the prefrontal cortex. Learn Mem, 14(3), 117-125.

Nagai, Y., Critchley, H. D., Featherstone, E., Fenwick, P. B., Trimble, M. R., \& Dolan, R. J. (2004). Brain activity relating to the contingent negative variation: an fMRI investigation. Neuroimage, 21(4), 12321241.

Nathan, P. J., Harrison, B. J., Olver, J. S., Norman, T. R., Burrows, G. D., \& Stough, C. (2002). Depletion of Serotonin versus Dopamine Produces Double Dissociation on Tests of Mnemonic Function in Healthy Volunteers. Int J Neuropsychopharmacol, 5 (Suppl 1), S191.

Naylor, H., Halliday, R., \& Callaway, E. (1985). The effect of methylphenidate on information processing. Psychopharmacology (Berl), 86(1-2), 90-95.

Nieoullon, A. (2002). Dopamine and the regulation of cognition and attention. Prog Neurobiol, 67(1), 53-83.

Nieoullon, A., \& Coquerel, A. (2003). Dopamine: a key regulator to adapt action, emotion, motivation and cognition. Curr Opin Neurol, 16 Suppl 2, S3-9.

Nuechterlein, K. H., Barch, D. M., Gold, J. M., Goldberg, T. E., Green, M. F., \& Heaton, R. K. (2004). Identification of separable cognitive factors in schizophrenia. Schizophr Res, 72(1), 29-39.

Nutt, J. G. (2008). Pharmacokinetics and pharmacodynamics of levodopa. Movement disorders : official journal of the Movement Disorder Society, 23 Suppl 3, S580-584.

Oei, N. Y., Tollenaar, M. S., Elzinga, B. M., \& Spinhoven, P. (2010). Propranolol reduces emotional distraction in working memory: a partial mediating role of propranolol-induced cortisol increases? Neurobiology of learning and memory, 93(3), 388-395.

Oishi, M., Mochizuki, Y., Du, C., \& Takasu, T. (1995). Contingent negative variation and movement-related cortical potentials in parkinsonism. Electroencephalogr Clin Neurophysiol, 95(5), 346-349.

Oken, B. S., Kishiyama, S. S., \& Salinsky, M. C. (1995). Pharmacologically induced changes in arousal: effects on behavioral and electrophysiologic measures of alertness and attention. Electroencephalogr Clin Neurophysiol, 95(5), 359-371.

Owen, A. M., Iddon, J. L., Hodges, J. R., Summers, B. A., \& Robbins, T. W. (1997). Spatial and non-spatial working memory at different stages of Parkinson's disease. Neuropsychologia, 35(4), 519-532.

Pacholczyk, T., Blakely, R. D., \& Amara, S. G. (1991). Expression cloning of a cocaine- and antidepressant-sensitive human noradrenaline transporter. Nature, 350(6316), 350-354. 
Papart, P., Ansseau, M., \& Timsit-Berthier, M. (1997). Influence of diazepam on Contingent Negative Variation. Hum Psychopharmacol(12), 95-98.

Patrick, K. S., Straughn, A. B., Minhinnett, R. R., Yeatts, S. D., Herrin, A. E., DeVane, C. L., et al. (2007). Influence of ethanol and gender on methylphenidate pharmacokinetics and pharmacodynamics. Clin Pharmacol Ther, 81(3), 346-353.

Paulus, W., \& Trenkwalder, C. (2006). Less is more: pathophysiology of dopaminergic-therapy-related augmentation in restless legs syndrome. Lancet neurology, 5(10), 878-886.

Pelosi, L., Hayward, M., \& Blumhardt, L. D. (1995). Is "memory-scanning" time in the Sternberg paradigm reflected in the latency of event-related potentials? Electroencephalogr Clin Neurophysiol, 96(1), 44-55.

Pietrzak, R. H., Mollica, C. M., Maruff, P., \& Snyder, P. J. (2006). Cognitive effects of immediate-release methylphenidate in children with attention-deficit/hyperactivity disorder. Neurosci Biobehav Rev, 30(8), 1225-1245.

Polich, J. (2007). Updating P300: an integrative theory of P3a and P3b. Clinical neurophysiology : official journal of the International Federation of Clinical Neurophysiology, 118(10), 2128-2148.

Polich, J. (2012). Neuropsychology of P300. In S. J. Luck \& E. S. Kappenman (Eds.), The Oxford Handbook of Event-Related Potential Components. Oxford: Oxford University Press.

Polich, J., \& Criado, J. R. (2006). Neuropsychology and neuropharmacology of $\mathrm{P} 3 \mathrm{a}$ and P3b. International journal of psychophysiology : official journal of the International Organization of Psychophysiology, 60(2), 172-185.

Prasher, D., \& Findley, L. (1991). Dopaminergic induced changes in cognitive and motor processing in Parkinson's disease: an electrophysiological investigation. J Neurol Neurosurg Psychiatry, 54(7), 603-609.

Pulvermuller, F., Lutzenberger, W., Muller, V., Mohr, B., Dichgans, J., \& Birbaumer, N. (1996). P3 and contingent negative variation in Parkinson's disease. Electroencephalogr Clin Neurophysiol, 98(6), 456467.

Ramaekers, J. G., Theunissen, E. L., de Brouwer, M., Toennes, S. W., Moeller, M. R., \& Kauert, G. (In press). Tolerance and cross-tolerance to neurocognitive effects of THC and alcohol in heavy cannabis users. Psychopharmacology (Berl), 214(2), 391-401.

Rektor, I., Bares, M., Kanovsky, P., Brazdil, M., Klajblova, I., Streitova, H., et al. (2004). Cognitive potentials in the basal ganglia-frontocortical circuits. An intracerebral recording study. Exp Brain Res, 158(3), 289301. 
Rektor, I., Kaiiovsky, P., Bares, M., Brazdil, M., Streitova, H., Klajblova, H., et al. (2003). A SEEG study of ERP in motor and premotor cortices and in the basal ganglia. Clin Neurophysiol, 114(3), 463-471.

Repantis, D., Schlattmann, P., Laisney, O., \& Heuser, I. (2010). Modafinil and methylphenidate for neuroenhancement in healthy individuals: A systematic review. Pharmacological research : the official journal of the Italian Pharmacological Society, 62(3), 187-206.

Riccio, C. A., Waldrop, J. J., Reynolds, C. R., \& Lowe, P. (2001). Effects of stimulants on the continuous performance test (CPT): implications for CPT use and interpretation. J Neuropsychiatry Clin Neurosci, 13(3), 326-335.

Riedel, W. J., Mehta, M. A., \& Unema, P. J. (2006). Human cognition assessment in drug research. Curr Pharm Des, 12(20), 2525-2539.

Robbins, T. W. (2005). Chemistry of the mind: neurochemical modulation of prefrontal cortical function. J Comp Neurol, 493(1), 140-146.

Rockstroh, B., Elbert, T., Lutzenberger, W., \& Altenmuller, E. (1991). Effects of the anticonvulsant benzodiazepine clonazepam on event-related brain potentials in humans. Electroencephalogr Clin Neurophysiol, 78(2), 142-149.

Roehrs, T., Papineau, K., Rosenthal, L., \& Roth, T. (1999). Sleepiness and the reinforcing and subjective effects of methylphenidate. Exp Clin Psychopharmacol, 7(2), 145-150.

Rogers, R. D., Blackshaw, A. J., Middleton, H. C., Matthews, K., Hawtin, K., Crowley, C., et al. (1999). Tryptophan depletion impairs stimulusreward learning while methylphenidate disrupts attentional control in healthy young adults: implications for the monoaminergic basis of impulsive behaviour. Psychopharmacology (Berl), 146(4), 482-491.

Roiser, J. P., McLean, A., Ogilvie, A. D., Blackwell, A. D., Bamber, D. J., Goodyer, I., et al. (2005). The subjective and cognitive effects of acute phenylalanine and tyrosine depletion in patients recovered from depression. Neuropsychopharmacology, 30(4), 775-785.

Ruhe, H. G., Mason, N. S., \& Schene, A. H. (2007). Mood is indirectly related to serotonin, norepinephrine and dopamine levels in humans: a metaanalysis of monoamine depletion studies. Mol Psychiatry, 12(4), 331359.

Rush, C. R., Essman, W. D., Simpson, C. A., \& Baker, R. W. (2001). Reinforcing and subject-rated effects of methylphenidate and $d$ amphetamine in non-drug-abusing humans. J Clin Psychopharmacol, 21(3), 273-286.

Rush, C. R., Kollins, S. H., \& Pazzaglia, P. J. (1998). Discriminative-stimulus and participant-rated effects of methylphenidate, bupropion, and 
triazolam in d-amphetamine-trained humans. Exp Clin Psychopharmacol, 6(1), 32-44.

Sahakian, B., \& Morein-Zamir, S. (2007). Professor's little helper. Nature, 450(7173), 1157-1159.

Sams-Dodd, F. (2005). Target-based drug discovery: is something wrong? Drug discovery today, 10(2), 139-147.

Schendan, H. E., Ganis, G., \& Kutas, M. (1998). Neurophysiological evidence for visual perceptual categorization of words and faces within $150 \mathrm{~ms}$. Psychophysiology, 35(3), 240-251.

Schoenen, J., Maertens de Noordhout, A., Timsit-Berthier, M., \& Timsit, M. (1986). Contingent negative variation and efficacy of beta-blocking agents in migraine. Cephalalgia : an international journal of headache, 6(4), 229-233.

Schroeder, S. R., Mann-Koepke, K., Gualtieri, C. T., Eckerman, D. A., \& Breese, G. R. (1987). Methylphenidate affects strategic choice behavior in normal adult humans. Pharmacol Biochem Behav, 28(2), 213-217.

Seeman, P., \& Madras, B. (2002). Methylphenidate elevates resting dopamine which lowers the impulse-triggered release of dopamine: a hypothesis. Behavioural brain research, 130(1-2), 79-83.

Semlitsch, H. V., Anderer, P., Schuster, P., \& Presslich, O. (1986). A solution for reliable and valid reduction of ocular artifacts, applied to the P300 ERP. Psychophysiology, 23(6), 695-703.

Shohamy, D., Myers, C. E., Geghman, K. D., Sage, J., \& Gluck, M. A. (2006). L-dopa impairs learning, but spares generalization, in Parkinson's disease. Neuropsychologia, 44(5), 774-784.

Shohamy, D., Myers, C. E., Grossman, S., Sage, J., \& Gluck, M. A. (2005). The role of dopamine in cognitive sequence learning: evidence from Parkinson's disease. Behavioural brain research, 156(2), 191-199.

Smith, J. L., Johnstone, S. J., \& Barry, R. J. (2006). Effects of pre-stimulus processing on subsequent events in a warned Go/NoGo paradigm: response preparation, execution and inhibition. Int J Psychophysiol, 61(2), 121-133.

Smith, J. L., Johnstone, S. J., \& Barry, R. J. (2007). Response priming in the Go/NoGo task: the N2 reflects neither inhibition nor conflict. Clin Neurophysiol, 118(2), 343-355.

Smith, M. E., \& Farah, M. J. (2011). Are prescription stimulants "smart pills"? The epidemiology and cognitive neuroscience of prescription stimulant use by normal healthy individuals. Psychological bulletin, 137(5), 717-741. 
Smulders, F. T., Kok, A., Kenemans, J. L., \& Bashore, T. R. (1995). The temporal selectivity of additive factor effects on the reaction process revealed in ERP component latencies. Acta Psychol (Amst), 90(1-3), 97-109.

Sobczak, S., Riedel, W. J., Booij, I., Aan Het Rot, M., Deutz, N. E., \& Honig, A. (2002). Cognition following acute tryptophan depletion: difference between first-degree relatives of bipolar disorder patients and matched healthy control volunteers. Psychol Med, 32(3), 503-515.

Soetens, E., Casaer, S., D'Hooge, R., \& Hueting, J. E. (1995). Effect of amphetamine on long-term retention of verbal material. Psychopharmacology (Berl), 119(2), 155-162.

Soetens, E., D'Hooge, R., \& Hueting, J. E. (1993). Amphetamine enhances human-memory consolidation. Neurosci Lett, 161(1), 9-12.

Spencer, T. J., Biederman, J., Ciccone, P. E., Madras, B. K., Dougherty, D. D., Bonab, A. A., et al. (2006). PET study examining pharmacokinetics, detection and likeability, and dopamine transporter receptor occupancy of short- and long-acting oral methylphenidate. Am J Psychiatry, 163(3), 387-395.

Stanzione, P., Fattapposta, F., Giunti, P., D'Alessio, C., Tagliati, M., Affricano, C., et al. (1991). P300 variations in parkinsonian patients before and during dopaminergic monotherapy: a suggested dopamine component in P300. Electroencephalogr Clin Neurophysiol, 80(5), 446453.

Sternberg, S. (1966). High-speed scanning in human memory. Science, 153(736), 652-654.

Stix, G. (2009). Turbocharging the brain. Scientific American Mind.

Stoops, W. W., Lile, J. A., Fillmore, M. T., Glaser, P. E., \& Rush, C. R. (2005). Reinforcing effects of methylphenidate: influence of dose and behavioral demands following drug administration. Psychopharmacology (Berl), 177(3), 349-355.

Strauss, J., Lewis, J. L., Klorman, R., Peloquin, L. J., Perlmutter, R. A., \& Salzman, L. F. (1984). Effects of methylphenidate on young adults' performance and event-related potentials in a vigilance and a pairedassociates learning test. Psychophysiology, 21(6), 609-621.

Studer, P., Wangler, S., Diruf, M. S., Kratz, O., Moll, G. H., \& Heinrich, H. (2010). ERP effects of methylphenidate and working memory load in healthy adults during a serial visual working memory task. Neurosci Lett, 482(2), 172-176.

Swaab, T. Y., Ledoux, K., Camblin, C. C., \& Boudewyn, M. A. (2012). Language-Related ERP Components. In S. J. Luck \& E. S. Kappenman 
(Eds.), The Oxford Handbook of Event-Related Potential Components. Oxford: Oxford University Press.

Swanson, J. M., \& Volkow, N. D. (2008). Increasing use of stimulants warns of potential abuse. Nature, 453, 586.

Tecce, J. J. (1972). Contingent negative variation (CNV) and psychological processes in man. Psychol Bull, 77(2), 73-108.

Tecce, J. J. (1991). Dopamine and CNV: studies of drugs, disease and nutrition. Electroencephalogr Clin Neurophysiol Suppl, 42, 153-164.

Tecce, J. J., \& Cole, J. O. (1974). Amphetamine effects in man: paradoxical drowsiness and lowered electrical brain acitivity (CNV). Science, 185(149), 451-453.

Tecce, J. J., Cole, J. O., \& Savignano-Bowman, J. (1975). Chlorpromazine effects on brain activity (contingent negative variation) and reaction time in normal women. Psychopharmacologia, 43(3), 293-295.

Timsit-Berthier, M. (1991). Contingent negative variation (CNV) in psychopharmacology. Electroencephalogr Clin Neurophysiol Suppl, 42, 142-152.

Timsit-Berthier, M., Mantanus, H., Marissiaux, P., Ansseau, M., Doumont, A., Geenen, V., et al. (1986). CNV and dopamine receptor reactivity: correlations with the apomorphine test. Electroencephalography and clinical neurophysiology. Supplement, 38, 403-405.

Tomasi, D., Volkow, N. D., Wang, G. J., Wang, R., Telang, F., Caparelli, E. C., et al. (2011). Methylphenidate enhances brain activation and deactivation responses to visual attention and working memory tasks in healthy controls. Neuroimage, 54(4), 3101-3110.

Townsend, J., Adamo, M., \& Haist, F. (2006). Changing channels: an fMRI study of aging and cross-modal attention shifts. Neuroimage, 31(4), 1682-1692.

Unrug, A., Coenen, A., \& van Luijtelaar, G. (1997). Effects of the tranquillizer diazepam and the stimulant methylphenidate on alertness and memory. Neuropsychobiology, 36(1), 42-48.

van Boxtel, G. J., \& Brunia, C. H. (1994). Motor and non-motor aspects of slow brain potentials. Biol Psychol, 38(1), 37-51.

van Deursen, J. A., Vuurman, E. F., Smits, L. L., Verhey, F. R., \& Riedel, W. J. (2009). Response speed, contingent negative variation and P300 in Alzheimer's disease and $\mathrm{MCl}$. Brain and cognition, 69(3), 592-599.

van Eijk, H. M., Rooyakkers, D. R., \& Deutz, N. E. (1993). Rapid routine determination of amino acids in plasma by high-performance liquid chromatography with a 2-3 microns Spherisorb ODS II column. J Chromatogr, 620(1), 143-148. 
van Ruitenbeek, P., Sambeth, A., Vermeeren, A., Young, S. N., \& Riedel, W. J. (2009). Effects of L-histidine depletion and L-tyrosine/Lphenylalanine depletion on sensory and motor processes in healthy volunteers. British journal of pharmacology, 157(1), 92-103.

van Stegeren, A. H., Goekoop, R., Everaerd, W., Scheltens, P., Barkhof, F., Kuijer, J. P., et al. (2005). Noradrenaline mediates amygdala activation in men and women during encoding of emotional material. Neuroimage, 24(3), 898-909.

Verleger, R., Paehge, T., Kolev, V., Yordanova, J., \& Jaskowski, P. (2006). On the relation of movement-related potentials to the go/no-go effect on P3. Biol Psychol, 73(3), 298-313.

Verleger, R., Wascher, E., Arolt, V., Daase, C., Strohm, A., \& Kompf, D. (1999). Slow EEG potentials (contingent negative variation and postimperative negative variation) in schizophrenia: their association to the present state and to Parkinsonian medication effects. Clin Neurophysiol, 110(7), 1175-1192.

Volkow, N. D., Fowler, J. S., Wang, G. J., Ding, Y. S., \& Gatley, S. J. (2002). Role of dopamine in the therapeutic and reinforcing effects of methylphenidate in humans: results from imaging studies. Eur Neuropsychopharmacol, 12(6), 557-566.

Volkow, N. D., Fowler, J. S., Wang, G. J., Telang, F., Logan, J., Wong, C., et al. (2008). Methylphenidate decreased the amount of glucose needed by the brain to perform a cognitive task. PLoS ONE, 3(4), e2017.

Volkow, N. D., Wang, G. J., Fowler, J. S., Gatley, S. J., Logan, J., Ding, Y. S., et al. (1998). Dopamine transporter occupancies in the human brain induced by therapeutic doses of oral methylphenidate. $A m \mathrm{~J}$ Psychiatry, 155(10), 1325-1331.

Volkow, N. D., Wang, G. J., Fowler, J. S., Hitzemann, R., Gatley, J., Ding, Y. S., et al. (1998). Differences in regional brain metabolic responses between single and repeated doses of methylphenidate. Psychiatry Res, 83(1), 29-36.

Volkow, N. D., Wang, G. J., Gatley, S. J., Fowler, J. S., Ding, Y. S., Logan, J., et al. (1996). Temporal relationships between the pharmacokinetics of methylphenidate in the human brain and its behavioral and cardiovascular effects. Psychopharmacology (Berl), 123(1), 26-33.

Vrshek-Schallhorn, S., Wahlstrom, D., Benolkin, K., White, T., \& Luciana, M. (2006). Affective bias and response modulation following tyrosine depletion in healthy adults. Neuropsychopharmacology, 31(11), 25232536.

Walter, W. G., Cooper, R., Aldridge, V. J., McCallum, W. C., \& Winter, A. L. (1964). Contingent Negative Variation: An Electric Sign of 
Sensorimotor Association and Expectancy in the Human Brain. Nature, 203, 380-384.

Wascher, E., Verleger, R., Vieregge, P., Jaskowski, P., Koch, S., \& Kompf, D. (1997). Responses to cued signals in Parkinson's disease. Distinguishing between disorders of cognition and of activation. Brain : a journal of neurology, 120 ( Pt 8), 1355-1375.

Weerts, T. C., \& Lang, P. J. (1973). The effects of eye fixation and stimulus and response location on the contingent negative variation (CNV). Biological psychology, 1(1), 1-19.

Wetzel, C. D., Squire, L. R., \& Janowsky, D. S. (1981). Methylphenidate impairs learning and memory in normal adults. Behav Neural Biol, 31(4), 413-424.

Young, S. N., Smith, S. E., Pihl, R. O., \& Ervin, F. R. (1985). Tryptophan depletion causes a rapid lowering of mood in normal males. Psychopharmacology (Berl), 87(2), 173-177.

Zank, R., Strehl, U., Larbig, W., \& Kotchoubey, B. (2008). Effects of rizatriptan on the contingent negative variation in healthy women. Cephalalgia, 28(9), 922-932.

Zeeuws, I., Deroost, N., \& Soetens, E. (2010). Verbal memory improved by Damphetamine: influence of the testing effect. Hum Psychopharmacol, 25(5), 377-387.

Zeeuws, I., \& Soetens, E. (2007). Verbal memory performance improved via an acute administration of D-amphetamine. Hum Psychopharmacol, 22(5), 279-287. 
SUMMARY 
Dopamine is a neurotransmitter that is involved in several neuropsychiatric conditions with cognitive impairment including Parkinson's Disease, schizophrenia and ADHD. As recent advances in pharmacological treatment of these conditions have been disappointing, psychopharmacological research in this area continues to be highly relevant. A first step in the improvement of treatment of dopamine-associated diseases is to increase our knowledge about the dopamine system. Therefore, the first part of this dissertation aimed to determine the role of dopamine in cognitive function in the healthy population, primarily focusing on (working) memory.

Furthermore, research aimed at advancing pharmacological treatment of diseases in which dopamine is involved would be aided by a biomarker for dopamine activity. This dissertation proposed the use of contingent negative variation (CNV) amplitude as a marker of dopamine activity in the basal ganglia. Such a marker could be used to quickly verify whether interventions that are thought to elevate dopamine in that region actually do so. Therefore, the second part of this dissertation aimed to validate the CNV as a dopaminergic biomarker reflecting changed activity in the midbrain. To this end experiments were designed in which dopamine was deliberately manipulated

In the experimental studies described in this dissertation, dopamine activity and availability were experimentally elevated by administration of methylphenidate and levodopa respectively. Methylphenidate blocks the reuptake of dopamine and noradrenaline. Levodopa is the precursor of dopamine. A reduction of dopamine activity was achieved by depleting the brain of the dopamine precursors tyrosine and phenylalanine.

Part 1 discussed the role of dopamine in cognition. In chapter 1 the available literature on cognitive effects of methylphenidate in healthy volunteers was reviewed. In this review the extent to which methylphenidate enhanced cognitive performance in a certain domain was quantified by a percentage that reflected the number of studies showing positive effects of methylphenidate. To evaluate whether the dose-response relationship followed an inverted-U-shaped curve, methylphenidate effects on cognition were also quantified for low, medium and high doses respectively. The studies reviewed showed that methylphenidate improved cognitive performance in the healthy population in the domains of working memory (68\%) and speed of processing (57\%), and to a lesser extent also improved verbal learning and memory $(34 \%)$, reasoning and problem solving $(18 \%)$ and attention and vigilance (15\%), but did not have an effect on visual learning and memory. Methylphenidate effects were shown to be dose dependent and the dose response relationship differed between cognitive domains. 
Chapter 2 described a study in which dose related effects of methylphenidate on declarative memory, working memory, attention and executive function were investigated in healthy young volunteers. In a placebo-controlled crossover design 19 male participants (mean age: 23.4) were tested after a single dose of 10,20 or $40 \mathrm{mg}$ of methylphenidate. Performance on the 30 minute delayed recall test of a word learning task improved by 20 and $40 \mathrm{mg}$ of methylphenidate. Methylphenidate also improved set shifting and stop signal task performance but did not affect spatial working memory or planning task performance. As this was the first study to report dose-related effects of methylphenidate on declarative memory consolidation in healthy volunteers, we aimed to replicate these findings in the study described in the next chapter.

Besides replicating the previous results from chapter 2, chapter 3 aimed to study the dopaminergic contribution to the methylphenidate effects, as methylphenidate inhibits the reuptake of both dopamine and noradrenaline. To this end, effects of $40 \mathrm{mg}$ of methylphenidate on memory performance were compared to $100 \mathrm{mg}$ of levodopa in a placebo-controlled crossover study with 30 healthy volunteers ( 20 males, 10 females, mean age: 20.7). Methylphenidate did not improve 30 minute delayed recall. However, performance on the 24 hour delayed recall was better when words were learned under influence of methylphenidate compared to placebo and levodopa. Methylphenidate and levodopa had opposite effects on (working) memory task performance and electrophysiological correlates. Together the results of chapter 2 and 3 suggested that memory enhancing effects of methylphenidate were more pronounced when the circumstances were more challenging. Furthermore, it is likely that the levodopa manipulation in chapter 3 did not lead to enhanced dopaminergic activity.

The last chapter of part 1 of this dissertation, Chapter $\mathbf{4}$, described a study on the effects of a reduction of dopamine activity achieved by tyrosine/phenylalanine depletion (TPD). In a double blind placebo-controlled crossover design, the effect of TPD on memory performance and its electrophysiological correlates was studied in 17 healthy volunteers (6 males, 11 females, mean age: 21.1$)$. Working memory performance was not affected by TPD. However, event-related potential (ERP) measures were affected by the treatment, indicating that TPD impaired stimulus processing during working memory performance. Performance on the word learning test was not impaired after TPD but alterations in ERP measures suggested adverse effects of TPD on memory-related processing.

Part 2 focused on psychomotor control and how it is mediated by dopamine. As a first step in the validation of the CNV paradigm, differences between several paradigms that elicit a CNV were studied in chapter 5. The 
CNV is a slow negative shift in the electroencephalogram and is observed during response preparation. In an effort to optimize the CNV paradigm, a task using dynamic stimuli was developed. In the first experiment, 19 healthy male volunteers (mean age: 23.4 ) were subjected to the classic Traffic light (TL) task and the new dynamic Lines task. In the Lines task, response time was faster and CNV amplitude was larger compared to the TL task. In the second experiment, 20 healthy participants (10 males, 10 females, mean age: 23.0) were tested on a Go/No-go version of the Lines task. Response times increased as the probability of response requirement decreased. CNV amplitude was larger when probability of response requirement was higher. Thus the Lines task promoted response preparation and was employed in subsequent pharmacological studies alongside the Traffic Light task.

To determine sensitivity of CNV amplitude to dopamine activity the study described in chapter 6 assessed CNV amplitude in 19 healthy male volunteers (mean age: 23.4) after administration of placebo or 10,20 or $40 \mathrm{mg}$ of methylphenidate. Methylphenidate dose-dependently increased CNV amplitude and decreased reaction times. These results indicated that dopamine availability increased response readiness as measured by the CNV paradigm. The CNV appeared to be a good candidate biomarker for assessing changes in dopaminergic function. However, because methylphenidate inhibits reuptake of dopamine but also of noradrenaline, it could not be stated with certainty whether these findings could be ascribed solely to increased dopamine levels.

Therefore, Chapter 7 aimed to determine selectivity of the CNV for dopamine activity. To investigate the specific dopaminergic influence on the CNV amplitude the effects of $40 \mathrm{mg}$ methylphenidate on CNV were compared to those of $100 \mathrm{mg}$ of the dopamine precursor levodopa in a placebocontrolled crossover study testing 30 healthy volunteers (20 males, 10 females, mean age: 20.7). Methylphenidate and levodopa had opposite effects on CNV amplitude and reaction time: methylphenidate increased CNV amplitude and decreased reaction time in comparison with levodopa. It was suggested that levodopa might not have led to enhanced dopamine activity or that combined dopamine- and noradrenaline reuptake inhibition may be essential to enhance response readiness and that methylphenidate effects may be more pronounced if conditions are more demanding.

In the general discussion the findings from the studies in this dissertation were reviewed to form a comprehensive view with respect to the two central aims of this thesis. The cognition enhancing effects of methylphenidate suggested that dopamine plays a role in cognitive functions such as verbal learning and memory, working memory, attention and speed of processing. Healthy individuals may particularly benefit from 
methylphenidate's enhancing effects under challenging circumstances. However, the evidence is limited by the fact that methylphenidate is not a pure dopaminergic drug and that the attempt to show that the effects were specifically dopamine dependent failed. This issue also played a role in the validation of the CNV as a dopaminergic biomarker. CNV possesses characteristics that are important for a biomarker, but the findings described in the present dissertation provide insufficient support to claim a role of the CNV as a biomarker that is specific for dopaminergic activity. 


\section{SAMENVATTING}


Dopamine is een neurotransmitter die betrokken is bij verschillende neuropsychiatrische aandoeningen waarbij sprake is van cognitieve beperkingen zoals de ziekte van Parkinson, schizofrenie en ADHD. Aangezien de mate van vooruitgang in farmacologische behandeling van deze aandoeningen de laatste tijd teleurstellend is geweest, blijft psychofarmacologisch onderzoek van groot belang. Een eerste stap in de verbetering van behandeling van dopamine gerelateerde ziekten is het vergroten van onze kennis van het dopamine systeem. Daarom heeft het eerste deel van dit proefschrift als doel te bepalen wat de rol van dopamine is in het cognitief functioneren van gezonde mensen, waarbij de focus ligt op het (werk)geheugen.

Daarnaast zou onderzoek met als doel farmacologische behandeling van ziekten waarbij dopamine betrokken is te verbeteren ook gebaat zijn bij het vinden van een biomarker van dopamine activiteit. In dit proefschrift wordt de amplitude van de Contingent Negative Variation (CNV) voorgesteld als marker van dopamine activiteit in de basale ganglia. Een marker zou gebruikt kunnen worden om sneller vast stellen of interventies die geacht worden het dopamine niveau te verhogen dit ook daadwerkelijk doen. Daarom is het doel van het tweede deel van dit proefschrift om de CNV te valideren als biomarker die veranderde dopamine activiteit in de midden-hersenen aangeeft. Met dit doel werden experimenten ontworpen waarin het dopamine niveau gemanipuleerd werd.

In de experimentele studies die in dit proefschrift beschreven worden, werd dopamine activiteit experimenteel verhoogd door toediening van metylfenidaat en levodopa. Metylfenidaat blokkeert de heropname van dopamine en noradrenaline. Levodopa is de precursor van dopamine. Een verlaging van dopamine activiteit werd bereikt door de beschikbaarheid van de dopamine grondstoffen tyrosine en fenylalanine te verminderen.

Deel 1 beschrijft de rol van dopamine in cognitie. In hoofdstuk 1 wordt de beschikbare literatuur over de cognitieve effecten van metylfenidaat in gezonde vrijwilligers beschreven. In dit hoofdstuk wordt de mate waarin metylfenidaat cognitieve prestaties binnen specifieke domeinen verbetert, uitgedrukt in een percentage dat weergeeft hoeveel studies positieve effecten van metylfenidaat lieten zien. Om na te gaan of de dosisresponse curve een omgekeerde $U$ vorm had, werden metylfenidaat effecten op cognitie ook gekwantificeerd voor respectievelijk lage, middelhoge en hoge doseringen. De studies lieten zien dat metylfenidaat cognitieve prestaties van de gezonde populatie verbeterde binnen de domeinen werkgeheugen $(68 \%)$, en verwerkingssnelheid $(57 \%)$, en in mindere mate ook verbaal leren en geheugen (34\%), redeneren en probleem oplossen (18\%) en aandacht en vigilantie (15\%), maar geen effect had op visueel leren en 
geheugen. Metylfenidaat effecten waren afhankelijk van de dosering en de vorm van de dosis-respons curve verschilde per cognitief domein.

Hoofdstuk 2 beschreef een experiment waarin dosis gerelateerde effecten van metylfenidaat op declaratief geheugen, werkheugen, aandacht en executief functioneren werden onderzocht in gezonde jonge vrijwilligers. In een placebo gecontroleerd experiment werden 19 mannelijke proefpersonen (gemiddelde leeftijd: 23.4) getest na een enkele dosering van 10, 20 of $40 \mathrm{mg}$ metylfenidaat. Prestatie op een geheugentest 30 minuten na een woordenleertaak verbeterde na inname van 20 en $40 \mathrm{mg}$ metylfenidaat. Metylfenidaat verbeterde ook de prestatie op de zogenaamde set-shifting en stop signal taken maar had geen effect op spatieel werkgeheugen of planning. Omdat dit de eerste studie was die dosis-gerelateerde effecten van metylfenidaat op consolidatie in het declaratief geheugen van gezonde vrijwilligers gerapporteerd heeft, stelden we ons als doel deze bevindingen te repliceren in een studie die in het volgende hoofdstuk werd besproken.

Behalve het repliceren van eerdere resultaten uit hoofdstuk 2 , had hoofdstuk 3 als doel om te bestuderen in welke mate dopamine bijdraagt aan de metylfenidaat effecten, aangezien metylfenidaat heropname van zowel dopamine als noradrenaline remt. Daarom werden de effecten van $40 \mathrm{mg}$ metylfenidaat op geheugenprestaties vergeleken met $100 \mathrm{mg}$ levodopa in een placebo gecontroleerde gebalanceerde studie met 30 gezonde vrijwilligers (20 mannen, 10 vrouwen, gemiddelde leeftijd: 20.7). Metylfenidaat leidde niet tot verbetering van prestatie op de geheugentest die 30 minuten na het leren werd afgenomen. Echter, na 24 uur was het geheugen voor woorden die onder invloed van metylfenidaat werden geleerd wel beter dan bij placebo en levodopa. Metylfenidaat en levodopa hadden tegengestelde effecten op (werk)geheugen en hun electrofysiologische correlaten. Samen wijzen de resultaten uit de hoofdstukken 2 en 3 erop dat geheugen verbeterende effecten van metylfenidaat duidelijker aanwezig zijn wanneer de omstandigheden uitdagender zijn. Het is bovendien waarschijnlijk dat de levodopa manipulatie uit hoofdstuk 3 niet heeft geleid tot verhoogde dopamine activiteit.

Het laatste hoofdstuk van deel 1 van dit proefschrift, hoofdstuk 4, beschreef een studie naar de effecten van een reductie van dopamine activiteit die bereikt werd door tyrosine/fenylalanine depletie (TFD). In een dubbelblind placebo gecontroleerd gebalanceerd design, werd het effect van TFD op geheugen en de electrofysiologische correlaten bestudeerd in 17 gezonde vrijwilligers (6 mannen, 11 vrouwen, gemiddelde leeftijd: 21.1). Werkgeheugen prestatie werd niet beïnvloed door TFD. Echter, Event-related Potentials (ERPs) werden wel beïnvloed door de behandeling, wat er op wees dat TFD stimulus verwerking tijdens werkgeheugen taken verslechterde. 
Prestatie op de woordenleertaak was niet aangetast na TFD maar veranderingen in de ERP maten suggereerden wel nadelige effecten van TFD op geheugen gerelateerde verwerkingsprocessen.

Deel 2 focuste op psychomotore controle en hoe deze gemedieerd wordt door dopamine. Als een eerste stap in de validatie van de CNV werden verschillen tussen paradigma's die een CNV oproepen bestudeerd in hoofdstuk 5. De CNV is een langzame negatieve golf in het electroencefalogram die geobserveerd wordt tijdens respons voorbereiding. In een poging om het CNV paradigma te optimaliseren werd er een taak met dynamische stimuli ontwikkeld. In het eerste experiment werden 19 gezonde mannelijke vrijwilligers (gemiddelde leeftijd 23.4) onderworpen aan de klassieke Verkeerslichttaak en de nieuwe dynamische Lijnentaak. In de Lijnentaak waren de responstijd en de CNV amplitude groter in vergelijking met de Verkeerslichttaak. In het tweede experiment werden 20 gezonde proefpersonen (10 mannen, 10 vrouwen, gemiddelde leeftijd: 23.0) getest op een Go/No-go versie van de lijnentaak. Responstijden werden langer naarmate de kans dat men moest reageren afnam. De lijnentaak bevorderde dus respons voorbereiding en zal in vervolg studies naast de Verkeerslichttaak gebruikt worden.

Om de gevoeligheid van de CNV amplitude voor dopamine activiteit te bepalen werden in de studie beschreven in hoofdstuk 6, 19 gezonde mannelijke vrijwilligers (gemiddelde leeftijd: 23.4) getest na toediening van placebo of 10,20 of $40 \mathrm{mg}$ metylfenidaat. Metylfenidaat vergrootte de CNV amplitude en verkortte reactie tijden op een dosisafhankelijke wijze. Deze resultaten wijzen erop dat dopamine beschikbaarheid de bereidheid te reageren, zoals gemeten met de CNV taak, verbetert. De CNV leek een goede kandidaat biomarker te zijn voor het meten van veranderingen in dopminerge functies. Echter, omdat metylfenidaat heropname van zowel dopamine als noradrenaline remt kan niet met zekerheid worden gezegd of deze bevindingen enkel en alleen aan verhoogde dopamine niveaus kunnen worden toegeschreven.

Daarom had hoofdstuk 7 als doel om de gevoeligheid van de CNV voor dopamine activiteit te bepalen. Om de specifieke dopaminerge invloed op de CNV amplitude te bepalen werden de effecten van $40 \mathrm{mg}$ metylfenidaat op de CNV vergeleken met die van $100 \mathrm{mg}$ van de dopamine precursor levodopa in een placebo gecontroleerde gekruiste studie waarin 30 gezonde vrijwilligers (20 mannen, 10 vrouwen, gemiddelde leeftijd: 20.7) werden getest. Metylfenidaat en levodopa hadden tegengestelde effecten op de CNV amplitude en reactietijd: metylfenidaat verhoogde de CNV amplitude en verkortte de reactietijd in vergelijking met levodopa. Er werd gesuggereerd dat levodopa ofwel niet tot een verhoging in dopamine activiteit had geleid 
ofwel dat gecombineerde dopamine- en noradrenaline heropname remming essentieel zou kunnen zijn voor het verbeteren van bereidheid te reageren. Daarnaast werd gesuggereerd dat metylfenidaat effecten duidelijker aanwezig zijn als de condities uitdagender zijn.

In de algemene discussie werden de bevindingen van dit proefschrift nader bekeken om zo een samenhangend beeld te krijgen van twee centrale doelstellingen van dit proefschrift. De cognitie verbeterende effecten van metylfenidaat suggereren dat dopamine een rol speelt in cognitieve functies als verbaal leren en geheugen, werkgeheugen, aandacht en verwerkingssnelheid. Gezonde individuen zouden in het bijzonder van metylfenidaat kunnen profiteren wanneer de omstandigheden uitdagend zijn. Echter, het bewijs is beperkt doordat metylfenidaat geen puur dopaminerge stof is en dat de poging om aan te tonen dat de effecten specifiek dopamine afhankelijk zijn gefaald heeft. Dit probleem speelde ook een rol bij de validatie van de CNV als dopaminerge biomarker. De CNV bezit eigenschappen die belangrijk zijn voor een biomarker, maar de bevindingen zoals beschreven in dit proefschrift vormen onvoldoende bewijs om te beweren dat de CNV een biomarker kan zijn die specifiek is voor dopaminerge activiteit. 


\section{DANKWOORD}


Dat ik een boek heb kunnen vullen met het werk van de afgelopen vier jaar heb ik mede te danken aan de hulp en steun van een heel veel mensen, die ik daarvoor op deze pagina's enorm wil bedanken.

Ten eerste wil ik mijn promotor Wim Riedel bedanken. Wim, ik vond het fijn dat je telkens tijd voor me maakte wanneer je in Maastricht was en mij daarnaast vanuit Basel of Cambridge van advies voorzag via e-mail of telefoon. Als beginnend onderzoeker merkte ik al gauw dat jij open stond voor mijn mening en ideeën. Jouw directe, opbouwende feedback heb ik altijd enorm gewaardeerd. Bedankt voor je betrokkenheid en goede begeleiding.

Een groot dankjewel ook aan mijn copromotoren. Anke S. je was een ontzettend fijne copromotor. Ik kon altijd bij jou terecht met al mijn grote en kleine vragen, waarop ik soms zelfs antwoord kreeg zonder ze te hoeven stellen. We bleken vaak op dezelfde golflengte te zitten (het zal wel door de naam komen....?!). Heel erg bedankt voor alle tijd en moeite die je in mij hebt geïnvesteerd. Ik vond het bijzonder fijn dat je mijn begeleider was. Eric, ook bij jou stond de deur altijd open voor mij. Je kwam altijd met concrete ideeën en aanbevelingen en hield daarbij sterk rekening met wat voor mij het beste was. Vooral op de momenten waarop ik het even niet zag zitten wist je mij gerust te stellen en opnieuw te motiveren. Bedankt voor het vertrouwen!

I would like to thank F. Hoffmann-La Roche Ltd, Basel, Switzerland for the financial and intellectual contribution to the studies in this thesis.

$\mathrm{Bij}$ de uitvoering van de onderzoeken waren er veel andere taken die vervuld moesten worden. Cees, bedankt voor alle medische keuringen en adviezen bij de screening van proefpersonen. Irma, door jou waren mijn proefpersonen altijd op tijd, dankjewel daarvoor. Elian, bedankt voor de administratieve ondersteuning. Anita, bedankt voor alle praktische hulp (kun je misschien even komen prikken?). Stagaires en student-assistenten Natalie, Conny, Lizzy, Irene, Saskia, Lena, Monique en Rutger: allemaal hartstikke bedankt voor jullie inzet. Zonder jullie had ik alle metingen nooit in dit tijdsbestek kunnen uitvoeren! ledereen die zijn steentje heeft bijgedragen, van de collega's van de ICT tot collega's die mijn taakjes wilden uitproberen, allemaal hartelijk bedankt! Alle proefpersonen wil ik bedanken voor hun medewerking.

Collega's van de afdeling NP\&PP: bedankt voor de leuke tijd. De dagjes uit, de gezamenlijke lunches en de vele vlaai-momenten: het was allemaal heel gezellig met jullie! Kim, Eef, Joep, Silke, Peter v. R. en Maria (you can read Dutch, right?), jullie waren bijzonder fijne collega's! Ellen, ik mis je.

Dat ik mij zo thuis voelde op de universiteit was voor een groot deel ook te danken aan mijn kamergenootjes Katja, Janelle, Eliza, Joy en in het 
bijzonder Wendy en Inge: ik vond het heel leuk om met jullie een kantoor te delen. Merci Joy for being such a nice roommate! Bedankt voor jullie luisterend oor en de goede sfeer op onze kamer (en Inge bedankt voor alle grappige You Tube filmpjes die je doorstuurde!).

I want to thank my colleagues from Cambridge. Luke, thank you for giving me the opportunity to visit your lab. Christoph, danke dir für unsere Zusammenarbeit, es war super! LARGE colleagues, thank you for making my time in Cambridge so pleasurable. Ruth, bedankt voor je geweldige gezelschap (waar was de rest toch altijd?). Ik hoop dat wij in de toekomst ooit opnieuw collega's worden.

Dankjewel aan mijn 'berschermengelen', mijn steun en toeverlaat tijdens de verdediging, oftewel mijn paranimfen, Conny en Ryanne. Conny, plotseling kwam ik je overal tegen, in de trein, op de uni en op de sportschool. Het valt blijkbaar ook andere mensen op dat we veel gemeen hebben - hoe vaak kregen we al de vraag 'Zijn jullie zusjes?'. Bedankt dat je altijd bereid bent om als klankbord te fungeren, bedankt voor je gezelschap op de sportschool (je bent een grote motiverende factor!) en bedankt dat je mijn paranimf wilde zijn.

Ryanne, sinds we beiden aan de universiteit gingen studeren hebben we heel wat gezellige uurtjes samen doorgebracht, vroeger met knutselen en skaten, tegenwoordig voornamelijk met kletsen. Ik weet zeker dat jouw positieve instelling een perfecte eigenschap is voor een paranimf! lk ben heel blij dat je mij bijstaat! Bedankt voor alle hulp, advies en gezelligheid.

Mijn vrienden, loopmaatjes en (schoon)familie wil ik bedanken voor hun belangstelling en steun. Het was heel prettig om afleiding te vinden bij zulke fijne mensen. Renee en Bram, lieve zus en broer(tje), bedankt voor jullie betrokkenheid en relativeringsvermogen. Lisanne, ik vond het heel leuk om een paar maandjes met je samen te wonen in Cambridge, je was een geweldige huisgenote!

Pap en mam, jullie hebben op allerlei manieren bijgedragen aan het ontstaan van dit boekje. Behalve een veilige thuishaven vormen jullie voor $\mathrm{mij}$ een voorbeeld van perfectionisme, ijver, geduld en doorzettingsvermogen. Lieve pap en mam, ik wil jullie heel graag bedanken voor het feit dat jullie er altijd voor mij zijn. Pap, bedankt voor je bijdrage aan het omslag.

Lieve Andy, ik ben zo blij met jou! Jouw humor, begrip en vertrouwen zijn heel heel fijn. Je bent mijn alles. Dankjewel! 


\section{CURRICULUM VitaE}

Anke Linssen werd geboren op 9 oktober 1984 te Kerkrade. In 2003 behaalde zij haar VWO diploma aan het Bernardinuscollege in Heerlen. Vervolgens studeerde zij psychologie aan de Universiteit Maastricht. Na het afsluiten van de Bachelor in Biologische Psychologie in 2006 volgde zij de Onderzoeksmaster Neuropsychologie. Als onderdeel daarvan liep ze stage bij het Universitätsklinikum Aachen (Duitsland) en de Universiteit Maastricht. Na het behalen van het Masterdiploma (cum laude) in 2008 werkte zij gedurende 2 maanden als onderzoeksassistent op de afdeling Neuropsychologie en Psychofarmacologie aan de Universiteit Maastricht waar zij in november 2008 begon aan haar promotie onderzoek naar de rol van dopamine in cognitie en psychomotoriek. Van september 2011 tot maart 2012 was zij te gast bij de afdeling Experimental Psychology van de Universiteit van Cambridge, Verenigd Koninkrijk. Momenteel zet zij haar onderzoek aan de Universiteit Maastricht voort als postdoc onderzoeker. 


\section{Publications}

Linssen, A. M. W., Sambeth, A., Riedel, W. J., Vuurman, E. F.P. M. Higher, faster, stronger: The effect of dynamic stimuli on response preparation and CNV amplitude. Behavioural Brain Research, Accepted.

Linssen, A. M., Vuurman, E. F., Sambeth, A., et al. (2012). Methylphenidate produces selective enhancement of declarative memory consolidation in healthy volunteers. Psychopharmacol, 221 (4), 611-619.

Linssen, A. M. W., Vuurman, E. F. P. M., Sambeth, A., Nave, S., Riedel, W. J. (2011). Contingent Negative Variation as a dopaminergic biomarker: Evidence from dose-related effects of methylphenidate. Psychopharmacol, 218(3), 533-542.

Linssen, A. M., Riedel, W. J., \& Sambeth, A. (2011). Effects of tyrosine/phenylalanine depletion on electrophysiological correlates of memory in healthy volunteers. J Psychopharmacol, 25(2), 230-238.

\section{Submitted}

Linssen, A. M. W., Sambeth, A., Vuurman, E. F.P. M. Riedel, W. J. Cognitive effects of methylphenidate in healthy volunteers: A review.

Linssen, A. M. W., Riedel, W. J, Vuurman, E. F. P. M., \& Sambeth, A. Effects of methylphenidate and levodopa on (working) memory performance and Event Related Potentials in healthy volunteers.

Linssen, A. M. W., Riedel, W. J, Sambeth, A., \& Vuurman, E. F. P. M. Effects of methylphenidate and levodopa on the contingent negative variation. 
